\title{
Estudos dos Efeitos de Solventes no Espectro de Absorção Eletrônica da Merocianina de Brooker e Derivados.
}

\author{
Marcus Vinícius Araújo Damasceno \\ Tese de doutorado apresen- \\ tada ao Instituto de Física \\ para a obtenção do tí- \\ tulo de Doutor em Ciências.
}

Orientadora: Prof. Kaline Rabelo Coutinho

\section{Banca Examinadora:}

Prof ${ }^{\mathrm{a}}$. Dr ${ }^{\mathrm{a}}$. Kaline Rabelo Coutinho (IFUSP)

Prof ${ }^{\mathrm{a}}$. Dr ${ }^{\mathrm{a}}$. Rosangela Itri (IFUSP)

Prof ${ }^{\mathrm{a}}$. Dr ${ }^{\mathrm{a}}$. Thaciana Valentina Malaspina Fileti (UNIFESP-SJC)

Prof. Dr. Herbert de Castro Georg (UFG)

Prof. Dr. Nelson Henrique Morgon (IQ-USP)

São Paulo, 2014 


\section{FICHA CATALOGRÁFICA}

Preparada pelo Serviço de Biblioteca e Informação do Instituto de Física da Universidade de São Paulo

Damasceno, Marcus Vinícius Araújo

Estudos dos Efeitos de Solventes no Espectro de

Absorção Eletrônica da Merocianina de Brooker e Derivados - São Paulo, 2015.

Tese (Doutorado) - Universidade de São Paulo.

Instituto de Física. Depto. de Física Geral

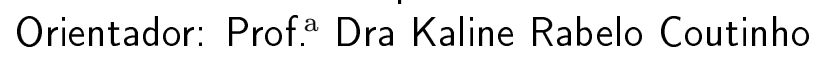

Área de Concentração: Física Atômica e Molecular

Unitermos: 1. Física molecular;

2. Efeitos do Solvente;

3. Simulações Computacionais;

4. Estrutura Eletrônica;

USP/IF/SBI-020/2015 
Dedico este trabalho à minha família pelo apoio e solidariedade. 


\section{Agradecimentos}

Gostaria de agradecer à todos que direta ou indiretamente contribuiram para a realização deste trabalho;

À Prof. ${ }^{a}$ Kaline Rabelo Coutinho, pela orientação, pelo agradável convívio e pela oportunidade de realizar este trabalho;

À toda minha familia, em especial aos meus pais Raimundo Damasceno e Maria de Lourdes pelo apoio incondicional;

Ao grupo de Modelagem Molecular, coordenado pelos professores Sylvio Canuto e Kaline Coutinho, pelas discussões em seminários/reuniões que contribuiram para a realização deste trabalho;

Ao professor Omar Abou El Seoud do Departamento de Química FundamentalInstituto de Química-USP pela doação da amostra de Merocianina de Brooker que possibilitou a realização de nossas medidas experimentais;

Aos doutores Cíntia C. Vequi-Suplicy e Antonio R. da Cunha pelo auxílio e acompanhamento nas medidas experimentais;

À professora Maria Teresa Lamy por ter disponibilizado a utilização dos equipamentos do laboratório de Biofísica do Instituto de Física da USP;

À FAPESP, pelo suporte e apoio financeiro na execução deste trabalho (processo 2009/11638-0). 



\section{Resumo}

Nesta tese estudamos o espectro de absorção da Merocianina de Brooker (MB) e três derivados em solventes com diferentes polaridades. O interesse no estudo desses sistemas se dá pela presença de algumas propriedades particulares das merocianinas, por exemplo, apresentam um grande deslocamento solvatocrômico devido à mudança na polaridade do meio.

Nós apresentamos os resultados teóricos obtidos para o espectro de absorção das moléculas considerando diferentes estruturas para o estado fundamental: formas trans, cis, zwiteriônica (zw), protonada $\left(\mathrm{MBH}^{+}\right)$e dímero $\left(\mathrm{MB}_{2}\right)$. O efeito do solvente foi considerado utilizando diferentes modelos de solvatação: (i) modelo contínuo polarizável (PCM), (ii) através de uma configuração eletrostática média do solvente (ASEC), (iii) incluindo algumas moléculas explícitas do solvente juntamente com um ambiente eletrostático gerado pelas demais moléculas do solvente. Como uma investigação adicional, apresentamos nossos resultados de medidas experimentais para o espectro de absorção da MB em vários solventes variando acidez e concentração.

Vimos, através de cálculos quânticos, que as formas cis/trans apresentam a excitação eletrônica na mesma região, e que a deformação estrutural gerada pela forma zw provoca um deslocamento para o vermelho na excitação eletrônica. Os nossos resultados teóricos e experimentais mostram que a forma $\mathrm{MBH}^{+}$apresenta um solvatocromismo pequeno, com deslocamento de $20 \mathrm{~nm}$ provocado pela mudança águaclorofórmio. Realizando medidas experimentais através da titulação espectroscópica nós obtivemos o $\mathrm{pK}_{a}$ associado ao processo de desprotonação/protonação da $\mathrm{MB}$ em água e metanol. Em água obtivemos um valor de 8.7, em boa concordância com valores da literatura. Apresentamos um valor inédito do $\mathrm{pK}_{a p}$ da MB em metanol, 9.9. Conseguimos uma boa descrição teórica para a excitação eletrônica da MB em solventes com alta polaridade, na região entre 430-500 nm, utilizando o método quântico TDDFT com funcionais B3LYP e CAM-B3LYP e conjunto de funções base 6-311+G**, porém a excitação em solventes de baixa polaridade, que ocorre na região entre 550-650 nm, não é corretamente descrita considerando a MB nos solventes. Nós vimos, através de estudos experimentais, que a sonda MB pode agregar em solventes de baixa polaridade. Os cáculos teóricos para dímeros em solução mostraram a existência de uma 
excitação eletrônica de baixa intensidade nesta região. Adicionalmente, os espectros experimentais em solventes de baixa polaridade mostraram 2 bandas, onde a segunda se assemelha com a banda observada para a forma $\mathrm{MBH}^{+}$. Para explicar essas 2 bandas experimentais para a MB, apresentamos uma proposta teórica onde ocorre uma transferência de prótons $\left(\mathrm{H}^{+}\right)$entre os monômeros do dímero formado, gerando uma estrutura desprotonada $\left(\mathrm{MB}^{-}\right)$e uma protonada $\left(\mathrm{MBH}^{+}\right)$. Cálculos teóricos para a forma $\mathrm{MB}^{-}$mostram que essa forma apresenta uma excitação eletrônica de intensidade moderada na região entre 550-650 nm. Com essa hipótese nós conseguimos descrever, através de cálculos teóricos, o solvatocromismo anômalo observado experimentalmente para o espectro eletrônico de absorção da MB nas duas regiões de polaridade dos solventes: alta polaridade, sendo descrita pela forma MB, e baixa polaridade, descrita pela forma $\mathrm{MB}^{-}$. 


\section{Abstract}

In this thesis we studied the absorption spectrum of merocyanine Brooker (MB) and three derivatives in solvents with different polarities. The interest in this system is given by the presence of some particular properties of this molecule, for example, it presents a large solvatochromic shift due to the change in the polarity of the medium.

We present the results for the absorption spectrum of the molecules considering different structures to the ground state: forms trans, cis, zwitterionic (zw), protonated $\left(\mathrm{MBH}^{+}\right)$and dimer $\left(\mathrm{MB}_{2}\right)$. The solvent effect was treaty by different ways: (i) continuous model using the PCM polarizable, (ii) by an average electrostatic configuration of solvent, ASEC, (iii) including some explicit solvent molecules with an electrostatic environment generated by other solvent molecules. As a additional investigation, we present results of experimental measurements in the thesis.

We have seen,through quantum calculations, that the forms cis/trans have the electronic excitation in the same region and the structural deformation generated in the $\mathrm{zw}$ form causes a red shift. Our theoretical and experimental results show that the $\mathrm{MBH}^{+}$form has a small solvatochromism, with displacement of $20 \mathrm{~nm}$ caused by water-chloroform change. Performing spectroscopic titration we got the $\mathrm{pK}_{a}$ associated with the process of deprotonation/protonation of MB in water and methanol. In water we obtained a value of 8.7 , in good agreement with the values reported in the literature. We present a unpublished $\mathrm{pK}_{a p}$ for the MB in methanol, 9.9. We have a good theoretical description for electronic excitation of MB in solvents with high polarity, in the region between 430-500 nm, using a method quantum TD-DFT B3LYP and CAM-B3LYP functional whit basic functions set $6-311+\mathrm{G}^{* *}$ but the excitation in low polarity solvents, which occurs in the region between $550-650 \mathrm{~nm}$, is not properly described considering the MB form in the solvents. We have seen, through experimental studies, that the MB probe can aggregate in low polarity solvents. Theoretical calculations for dimer in solution showed the existence of a low intensity electron excitation in this region. Additionally, the experimental spectra in low polarity solvents showed 2 bands, where in the second band is similar to the observed to $\mathrm{MBH}^{+}$form. To explain these two experimental bands for MB, we present a theoretical proposal where there is a proton transfer $\left(\mathrm{H}^{+}\right)$between the monomers of the dimer, generating 
a deprotonated structure $\left(\mathrm{MB}^{-}\right)$and a protonated $\left(\mathrm{MBH}^{+}\right)$. Theoretical calculations for the $\mathrm{MB}^{-}$show that this form presents an electronic excitation of moderate intensity in the region between 550-650 $\mathrm{nm}$. With this assumption we can describe, through theoretical calculations, the anomalous solvatochromism for the electronic absorption spectrum of MB in the two polarity regions of solvents: high polarity, is described by the $\mathrm{MB}$ form, and low polarity, described by the $\mathrm{MB}^{-}$form. 


\section{Sumário}

Lista de Abreviaturas vii

Lista de Figuras $\quad$ xi

$\begin{array}{ll}\text { Lista de Tabelas } & \text { xvii }\end{array}$

1 Introdução 1

1.1 Revisão bibliográfica: Merocianina de Brooker . . . . . . . . . . . . 9

1.2 Organização da Tese . . . . . . . . . . . . . . . . . . . 10

2 Métodos quânticos para representação do soluto. 13

2.1 Introdução . . . . . . . . . . . . . . . . . . . 13

2.2 Aproximação de Born-Oppenheimer . . . . . . . . . . . . . . 14

2.3 Método de Hartree-Fock . . . . . . . . . . . . . . . . . . . . . . 17

$2.3 .1 \quad$ Equação de Hartree-Fock-Roothaan . . . . . . . . . . . . . . . . 22

2.3 .2 Funções de Base . . . . . . . . . . . . . . . 25

2.4 Aproximações Perturbativas . . . . . . . . . . . . . . . . 26

2.4 .1 Teoria de Perturbação de Rayleigh-Schrödinger . . . . . . . . 26

2.4 .2 Teoria de Perturbação Møller-Plesset . . . . . . . . . . . . . . 31

2.5 Teoria do Funcional da Densidade $\ldots \ldots \ldots \ldots$

2.5 .1 Equações de Kohn-Sham . . . . . . . . . . . . . . . . 37

2.5 .2 DFT Dependente do Tempo (TD-DFT) . . . . . . . . . . . . 41

$2.6 \quad$ Ajuste das Cargas Parciais - Método Chelpg . . . . . . . . . . . . . . . 44

3 Métodos para inclusão dos efeitos do solvente. 49 


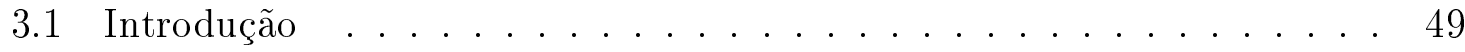

3.2 Método Quântico: Modelo Contínuo para o Solvente . . . . . . . . . . 49

3.3 Métodos Clássicos . . . . . . . . . . . . . . . . . 52

3.3 .1 Métodos de simulação . . . . . . . . . . . . . . . . 52

3.3 .2 Método Monte Carlo . . . . . . . . . . . . . . . . . 55

3.3 .3 Detalhes da Implementação … . . . . . . . . . . . . 61

3.3 .4 Potencial de Interação Intermolecular . . . . . . . . . . . . . . 63

3.3.5 Propriedades Estruturais: Função de Distribuição Radial . . . . 65

\begin{tabular}{|lll}
\hline 4 Metodologia experimental & 69
\end{tabular}

4.1 Materiais . . . . . . . . . . . . . . . . . . . . . . . . . 69

4.2 Preparação das amostras $\ldots \ldots \ldots \ldots \ldots$

4.2 .1 Preparação da Merocianina de Brooker em solução $\ldots . . .70$

4.2 .2 Procedimento de titulação com espectro UV-vis $\ldots \ldots$. . . . 70

4.2 .3 Diferentes concentrações do soluto . . . . . . . . . . . . . . 71

4.2 .4 Procedimento com Ultrassom . . . . . . . . . . . . . 72

5 Merocianina de Brooker: Resultados teóricos 75

5.1 Estudo da molécula isolada . . . . . . . . . . . . . . 75

5.1 .1 Geometrias . . . . . . . . . . . . . . . . . . . 75

5.1 .2 Espectro de Absorção $\ldots \ldots \ldots$. . . . . . . . . . . . . 79

5.1 .3 Efeito Solvente: modelo contínuo . . . . . . . . . . . . . . . 84

5.1 .4 Efeito Solvente: modelo discreto . . . . . . . . . . . . . . . 92

5.2 Dímero de MB . . . . . . . . . . . . . . . . . . . . . . . . 109

5.2 .1 Efeito Solvente: modelo contínuo . . . . . . . . . . . . . 109

5.2 .2 Efeito Solvente: modelo discreto . . . . . . . . . . . . . . . . 112

5.3 Determinação da constante de acidez . . . . . . . . . . . . . 116

6 Derivados da Merocianina de Brooker: Resultados teóricos 125

6.1 Molécula Isolada . . . . . . . . . . . . . . . . . . . . . 125

6.1 .1 Geometrias . . . . . . . . . . . . . . . . 126

6.1 .2 Espectro de Absorção . . . . . . . . . . . . . . . . . 135

6.2 Molécula em solução $\ldots$. . . . . . . . . . . . . . . . . . . . . . 139 
6.2.1 Efeito do solvente no espectro eletrônico de absorção: modelo

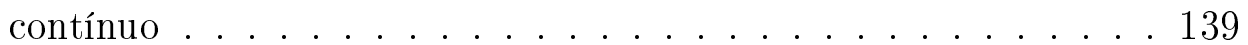

6.2.2 Efeito solvente na geometria e no espectro eletrônico: modelo

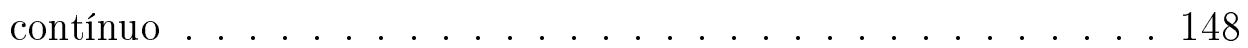

6.2.3 Efeito do solvente no espectro eletrônico de absorção: modelo

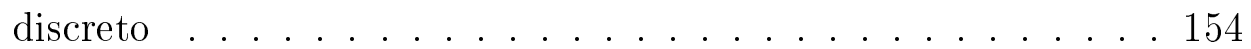

6.3 Dímeros em solução ～. . . . . . . . . . . . . . . . . . 166

6.3 .1 Simulação com dímeros . . . . . . . . . . . . . . . . . . . . 171

7 Merocianina de Brooker: Resultados experimentais $\quad 175$

7.1 Estudo do deslocamento solvatocrômico da Merocianina de Brooker . . 175

7.2 Titulação espectroscópica e determinação de pKa . . . . . . . . . . . . . 181

$7.2 .1 \quad$ Espectro de absorção: Água . . . . . . . . . . . . . . . 183

7.2 .2 Espectro de absorção: Metanol . . . . . . . . . . . . . . . 184

7.2 .3 Determinação da constante de acidez . . . . . . . . . . . . . 186

$7.3 \quad$ Estudo da agregação da sonda MB . . . . . . . . . . . . . . . . . 190

7.3 .1 Solvente: metanol . . . . . . . . . . . . . . . . . . 192

7.3 .2 Solvente: acetonitrila . . . . . . . . . . . . . . . . 197

7.4 Nossa proposta: transferência de prótons $\ldots$. . . . . . . . . . . . . 202

8 Conclusões 205

\begin{tabular}{ll}
\hline Referências Bibliográficas & 209
\end{tabular}

A Parâmetros Usados na Simulação. 225

A.1 Molécula neutra: MB. . . . . . . . . . . . . . . . . . . 225

A.2 Molécula protonada: $\mathrm{MBH}^{+}$. . . . . . . . . . . . . . . . . 232 


\section{Lista de Abreviaturas}

\begin{tabular}{ll} 
ASEC & Average Solvent Electrostatic Configuration \\
B3LYP & Becke's three-parameter exchange + Lee-Yang-Parr correlation \\
BHandHLYP & Becke's half-and-half exchange + Lee-Yang-Parr correlation \\
CHELPG & Charges from Electrostatic Potential Grid based \\
DFT & Teoria do Funcional da Densidade \\
E $_{T}$ & Energia de transição eletrônica \\
EOM-CCSD & Equation Of Motion-Coupled Cluster Singles and Doubles \\
GGA & Aproximação do Gradiente Generalizado \\
HF & Hartree-Fock \\
HK & Hohemberg e Kohn \\
HOMO & Orbital Molecular Mais Alto Ocupado \\
HUMO & Orbital Molecular Mais Baixo Desocupado \\
ICT & Intramolecular Charge Transfer \\
KS & Konh-Sham \\
LCAO & Combinação Linear de Orbitais Atômicos de Hidrogênio \\
LDA & Aproximação de Densidade de Spin-Local \\
\hline
\end{tabular}


MB

Merocianina de Brooker

$\mathrm{MBH}^{+} \quad$ Forma protonada da $\mathrm{MB}$

MC Monte Carlo

MDDF RDF da Mínima Distância entre os átomos do soluto e os átomos do solvente

$\mathrm{MeAMBr}_{2}$ 2,6-dibromo-4-[(E)-2-(1-methylacridinium-4-yl)ethenyl]phenolate

$\mathrm{MePMBr}_{2}$ 2,6-dibromo-4-[(E)-2-(1 methylpyridinium-4yl)ethenyl]phenolate

$\mathrm{MeQMBr}_{2}$ 2,6-dibromo-4-[(E)-2(1-methylquinolinium-4-yl)ethenyl]phenolate

MM Mecânica Molecular

MP Teoria de Perturbação Møller Plesset

MP2 Teoria de Perturbação de Møller Plesset de segunda ordem

PBE Perdew-Burke-Erzenhof

PCM Modelo Contínuo Polarizável

$\mathrm{pH}_{a p} \quad \mathrm{pH}$ aparente

$\mathrm{pK}_{a p} \quad \mathrm{pK}_{a}$ aparente

QM Mecânica Quântica

RDF $\quad$ Função de Distribuição Radial

SCF Campo Auto-Consistente

S-QM/MM Sequêncial- Mecânica Quântica/Mecânica Molecular

TD-DFT Teoria do Funcional da Densidade Dependente do Tempo

TPRS Teoria de Perturbação Rayleigh-Schrödinger

Zw Forma zwiteriônica 


\section{Lista de Figuras}

1.1 Ilustração da Merocianina de Brooker (MB). . . . . . . . . . . . . 5

1.2 Correlação entre $E_{T}(\mathrm{MB})$ e $E_{T}(30)$. Dados obtidos da referência |22|.

Os solventes que estão numerados nos gráficos são: água (1), metanol

(2), acetonitrila (3), piridina (4) e dioxano (5). A medida em dioxano

foi obtida pela extrapolação de misturas binárias de solventes. . . . . . 6

1.3 Derivados da MB que foram estudadas neste trabalho. (a) 2,6-dibromo4-[(E)-2-(1 methylpyridinium-4yl)ethenyl]phenolate $\left(\mathrm{MePMBr}_{2}\right) ;(\mathrm{b}) 2,6-$ dibromo-4-[(E)-2(1-methylquinolinium-4-yl)ethenyl]phenolate $\left(\mathrm{MeQMBr}_{2}\right)$; (c) 2,6-dibromo-4-[(E)-2-(1-methylacridinium-4-yl)ethenyl]phenolate $\left(\mathrm{MeAMBr}_{2}\right)$.

1.4 Correlação entre $E_{T}$ (sonda) e $E_{T}(30)$ para os 38 solventes estudados experimentalmente. Valores para $E_{T}$ foram obtidos da referência $|30|$.

Os solventes que estão numerados nos gráficos são: água (1), metanol

(2), DMSO (3), 1,4 dioxano (4), acetato de etila (5), e benzeno (6). . . 8

3.1 Ilustração da interação entre os sítios $i$ da molécula $a$ e os sítios $j$ da molécula $b$. Figura retirada da referência $\mid 99] \ldots \ldots \ldots 65$

3.2 Ilustração da representação dos picos da $G(r)$ correspondente. Curva correspondete à $G(r)_{O-O}$, para as sondas solvatadas em água. . . . . . 67 
5.1 Ilustração das estruturas da sonda MB que foram otimizadas, indicando a numeração utilizada nas análises seguintes. . . . . . . . . . . . . 76

5.2 Ilustração do dímero antiparalelo da $\mathrm{MB},\left(\mathrm{MB}_{2}\right)$.

5.3 Ilustração dos orbitais moleculares para as sonda MB obtidos com TDB3LYP $/ 6-311+\mathrm{G}^{* *} . \ldots \ldots \ldots \ldots \ldots$

5.4 Energia por molécula versus o número de ciclos MC durante o processo de termalização (a) e durante o equilíbrio (b). Estrutura neutra. . . . . 94

5.5 Função de distribuição radial de pares, soluto-água. G(r) (a) e MDDF

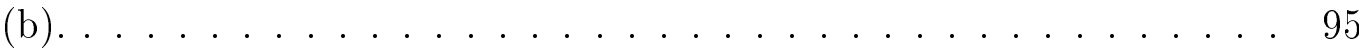

5.6 Função de distribuição radial de pares, solvente metanol. G(r) (a) e $\operatorname{MDDF}(\mathrm{b}) . \ldots \ldots \ldots \ldots \ldots 10 \ldots \ldots \ldots \ldots$

5.7 RDF de mínima distância, MDDF. Solvente acetonitrila. . . . . . . . 106

5.8 Ilustração dos orbitais moleculares envolvidos na transição eletrônica obtidos com TD-B3LYP/6-311+G** para o dímero de MB em metanol. 111

5.9 Energia de interação (em $\mathrm{kcal} / \mathrm{mol}$ ) do dímero $\mathrm{MB}_{2}$ ao longo da simulação MC em metanol. . . . . . . . . . . . . . . . . . . . . 113

5.10 Função de distribuição radial de pares, G(r). Solvente metanol. . . . . . 114

6.1 Ilustração das geometrias das sondas estudadas e a numeração dos átomos.126

6.2 Ilustração das geometrias das sondas estudadas na forma cis. . . . . . . 128

6.3 Ilustração das geometrias das sondas estudadas na forma zwiteriônica. Conformação trans. . . . . . . . . . . . . . . . . . . . . . 131

6.4 Ilustração da forma protonada para as sondas. Conformação trans . . . 134 
6.5 Energia de máxima absorção experimental, $E_{\max }$, para a sonda $\mathrm{MePMBr}_{2}$ versus a escala de polaridade de Reichardt normalizada, $E_{T}^{N}|30|$. Os solventes considerados foram: 1,4 Dioxano(1); (2) ; Acetonitrila(3); Cicloexano(4); 2-Metil-1-butanol(5); 2-Metoxietanol(6); Metanol(7) e

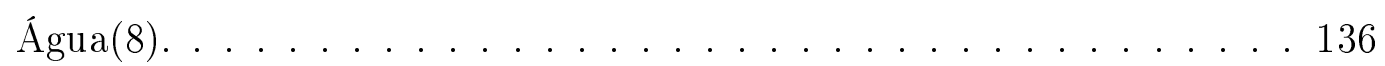

6.6 Ilustração dos orbitais moleculares para as sondas $\mathrm{MePMBr}_{2}, \mathrm{MeQMBr}_{2}$ \begin{tabular}{|c|c|c|}
\hline e $\mathrm{MeAMBr}_{2}$, respectivamente, obtidos com TD-B3LYP $/ 6-311+\mathrm{G}^{* *}$. \\
\hline
\end{tabular} Orbitais para as moléculas isoladas. . . . . . . . . . . . . . . 140

6.7 Comprimento das ligações C8-C9 e C15-O16, obtidos com B3LYP/6$311+\mathrm{G}^{* *}$, nos solventes: 1,4 dioxano $(\epsilon=2.21)$, metanol $(\epsilon=32.61)$, DMSO \begin{tabular}{|c|c|}
\hline$(\epsilon=46.83)$ e água $(\epsilon=78.36)$. Legenda do gráfico: (a) $\mathrm{MePMBr}_{2}$, (b) \\
\hline
\end{tabular} - $\mathrm{MeQMBr}_{2}$ e (c) $\mathrm{MeAMBr}_{2}$. . . . . . . . . . . . . . 148

6.8 Momento de dipolo para as sondas nos diferentes solventes, obtido \begin{tabular}{|c|}
\hline com MP2 $/ 6-311+\mathrm{G}^{*}$. Os solventes são: 1,4 dioxano $(\epsilon=2.21)$, metanol \\
\hline
\end{tabular} ( $(\epsilon=32.61)$, DMSO $(\epsilon=46.83)$ e água $(\epsilon=78.36) . \ldots \ldots \ldots$. . . . . 149

6.9 Ilustração dos solventes considerados nas simulações. . . . . . . . . . 155

6.10 Energia por molécula versus o número de ciclos durante o processo de termalização do sistema. Dados obtidos para a sonda $\mathrm{MePMBr}_{2}$ em 1,4

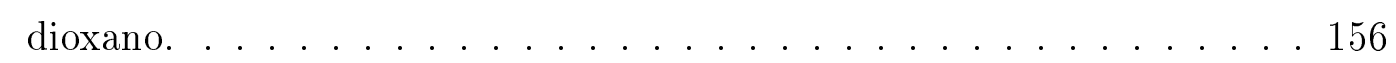

6.11 Energia por molécula versus o número de ciclos durante o equilíbrio

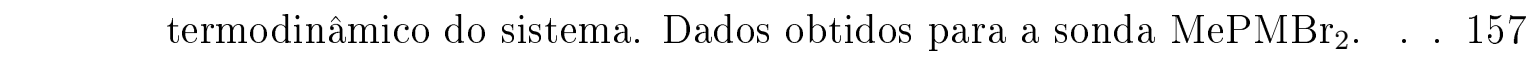

6.12 Função de distribuição radial de pares, $\mathrm{G}_{O O}(\mathrm{r})$, entre o oxigênio O16 das sondas e o oxigênio $\mathrm{O} 1$ dos solventes. . . . . . . . . . . . . 158

6.13 Função de distribuição de mínima distância entre o soluto e o solvente. 159

6.14 Função de distribuição radial de pares, $\mathrm{G}_{O O}(\mathrm{r})$, entre o oxigênio O16 das sondas e o oxigênio O1 dos solventes. . . . . . . . . . . . . . 161 
6.15 Função de distribuição de mínima distância entre o soluto e o solvente. 162

6.16 Ilustração dos dímeros antiparalelos otimizados das sondas. . . . . . . . 166

6.17 Ilustração dos orbitais moleculares envolvidos na transição eletrônica obtidos com TD-B3LYP/6-311+G** para o dímero de $\mathrm{MePMBr}_{2}$. . . . 169

6.18 Ilustração dos orbitais moleculares envolvidos na transição eletrônica obtidos com TD-B3LYP/6-311+G** para o dímero de $\mathrm{MeQMBr}_{2}$. . . . 170

6.19 Ilustração dos orbitais moleculares envolvidos na transição eletrônica obtidos com TD-B3LYP/6-311+G** para o dímero de $\mathrm{MeAMBr}_{2}$. . . . 170

6.20 Função de distribuição radial de pares, G(r). . . . . . . . . . . . . . . 172

6.21 Energia de interação entre as duas moléculas do soluto, U(kcal/mol), ao longo da etapa de produção para a sonda $\mathrm{MePMBr}_{2}$. . . . . . . . . . 173

7.1 Espectro eletrônico de absorção da sonda MB medido em diferentes solventes. ............................ 176

7.2 Medidas para a absorbância normalizada da sonda MB em diferentes solventes utilizando a solução estoque ácida. Os solventes utilizados foram: água $\left(\lambda_{\max }=374 \mathrm{~nm}\right)$, DMSO $\left(\lambda_{\max }=393 \mathrm{~nm}\right)$, acetonitrila $\left(\lambda_{\max }=383 \mathrm{~nm}\right)$, metanol $\left(\lambda_{\max }=382 \mathrm{~nm}\right)$ e clorofórmio $\left(\lambda_{\max }=397\right.$

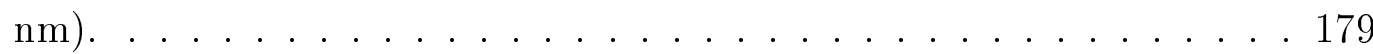

7.3 Medidas para a absorbância normalizada da sonda MB em diferentes solventes utilizando a solução estoque alcalina. Os solventes utilizados foram: água $\left(\lambda_{\max }=444 \mathrm{~nm}\right)$, DMSO $\left(\lambda_{\max }=572\right.$ e $\left.391 \mathrm{~nm}\right)$, acetonitrila $\left(\lambda_{\max }=568\right.$ e $\left.387 \mathrm{~nm}\right)$, metanol $\left(\lambda_{\max }=484 \mathrm{~nm}\right)$ e clorofórmio $\left(\lambda_{\max }=615\right.$ e $\left.408 \mathrm{~nm}\right) . \ldots \ldots \ldots$

7.4 Efeito do pH sobre o espectro eletrônico de absorção da sonda MB em água à concentração de 0.015 mM. . . . . . . . . . . . . . . . . . . 183 
7.5 $\quad$ Efeito do $\mathrm{pH}_{a p}$ sobre o espectro eletrônico de absorção da sonda MB em metanol. A concentração da sonda considerada foi de $0.015 \mathrm{mM}$. . . . . 185

7.6 Comportamento da absorbância da sonda MB em água variando o pH do meio nos comprimentos de onda: (a) $371 \mathrm{~nm}$ e (b) $444 \mathrm{~nm}$. Apresentamos o ajuste dos pontos experimentais considerando a equação

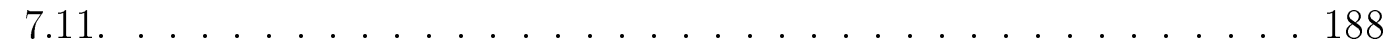

7.7 Comportamento da absorbância da sonda MB em metanol variando o $\mathrm{pH}_{a p}$ do meio nos comprimentos de onda: (a) $390 \mathrm{~nm}$ e (b) 485 nm. Apresentamos o ajuste dos pontos experimentais considerando a equação 7.11 . . . . . . . . . . . . . . . . . . 189

7.8 Espectro eletrônico de absorção da sonda MB em solução aquosa variando a concentração do soluto. Solução alcalina, $\mathrm{pH}=13.5$. . . . . . . . . 191

7.9 Comportamento da absorbância da sonda MB em solução aquosa variando a concentração do soluto. Solução alcalina, $\mathrm{pH}=13.5$. . . . . . . . . 191

7.10 Espectro eletrônico de absorção da sonda MB em solução de metanol variando a concentração do soluto. Solução ácida, $\mathrm{pH}_{a p}=0.3$. $\mathrm{Ab}$ sorbâncias medidas (a) e normalizada (b). . . . . . . . . . . . . . . 193

7.11 Comportamento da absorbância da sonda MB em solução de metanol variando a concentração do soluto. Solução ácida, $p H_{a p}=0.3 . \quad$. . . . . 194

7.12 Espectro eletrônico de absorção da sonda MB em solução de metanol variando a concentração do soluto. Solução alcalina, $p H_{a p}=12.6$. Absorbâncias medidas (a) e normalizadas (b). . . . . . . . . . . . 195

7.13 Comportamento da absorbância da sonda MB em solução de metanol variando a concentração do soluto. Solução alcalina, $\mathrm{pH}_{a p}=12.6$. . . . 195 
7.14 Comportamento da absorbância da sonda MB em solução de acetonitrila variando a concentração do soluto. Solução alcalina, $\mathrm{pH}_{a p}=11.0$. Absorbâncias medidas (a) e normalizadas (b). . . . . . . . . . . . . . 197

7.15 Comportamento da absorbância da sonda MB em solução de acetonitrila versus a concentração do soluto. Absorbâncias obtidas para as duas

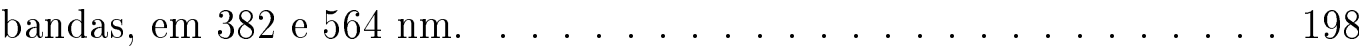

7.16 Espectro eletrônico de absorção da sonda MB em acetonitrila para duas soluções estoques diferentes: ácida e alcalina. . . . . . . . . . . . . . . . 199

7.17 Espectro eletrônico de absorção da sonda MB em acetonitrila para duas concentrações diferentes. Solução estoque alcalina.(a) absorbância normalizada para a banda em $385 \mathrm{~nm}$, (b) absorbância normalizada para a banda em $568 \mathrm{~nm} . \ldots \ldots$. . . . . . . . . . . . . 200

7.18 Espectro eletrônico de absorção da sonda MB em acetonitrila (amostra obtida a partir da solução estoque alcalina) antes e depois do ultrasom em diferentes intervalos de tempos. . . . . . . . . . . . . . . . . . 201

7.19 Ilustração da transferência de próton intradímeros gerando a forma tautomérica da MB, $\mathrm{MB}_{t} . \ldots \ldots \ldots \ldots . \ldots \ldots 20 \ldots$

7.20 Ilustração da transferência de próton intradímeros gerando as formas $\mathrm{MB}^{-} \mathrm{e} \mathrm{MBH}^{+}$

8.1 Comportamento anômalo para o solvatocromismo da MB. . . . . . . . 207 


\section{Lista de Tabelas}

5.1 Valores para o comprimento da ligação $(\AA)$, momento de dipolo (D), diferenças de energia relativa à conformação trans $\Delta \mathrm{E}(\mathrm{kcal} / \mathrm{mol}), \mathrm{com}-$ primento de onda de máxima absorção $\left(\lambda_{\max } \mathrm{em} \mathrm{nm}\right)$ e a força do oscilador da excitação eletrônica em parênteses para a sonda MB nas di-

ferentes estruturas. Valores obtidos para a molécula isolada, otimizada \begin{tabular}{|c|}
\hline em nível B3LYP $/ 6-311+\mathrm{G}^{* *}$. A numeração dos átomos na tabela está \\
\hline
\end{tabular}

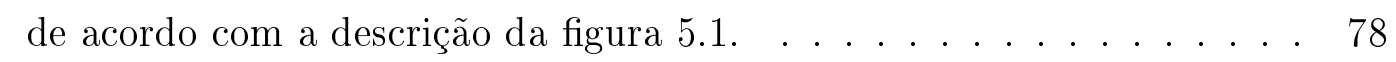

5.2 Valores da primeira energia de transição eletrônica $\left(\mathrm{em} \mathrm{cm}^{-1}\right)$, comprimento de onda (em nm) e força do oscilador (OSC) calculados para sonda trans-MB em vácuo com diferentes métodos de mecânica quântica

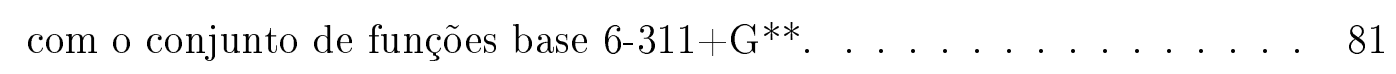

5.3 Valores para o comprimento da ligação $(\AA)$ para a sonda MB em diferentes solventes. Entre parênteses apresentamos as informações para a molécula protonada. A geometria foi otimizada em nível B3LYP/6$311+\mathrm{G}^{* *}$ e o solvente considerado através do modelo contínuo polarizável PCM. A numeração dos átomos na tabela segue a descrição da figura 5.1 ........................... 86 
5.4 Valores do momento de dipolo (D) e carga atômica (e) para a sonda MB em diferentes solventes. Geometria otimizada em nível B3LYP/6$311+\mathrm{G}^{* *}$ e momento de dipolo obtido com MP2 $/ 6-311+\mathrm{G}^{* *}$. A numeração dos átomos na tabela segue a descrição da figura 1.1 . . . . . . . . 87

5.5 Valores para energia de transição $\left(\mathrm{em} \mathrm{cm}^{-1}\right)$, comprimento de onda da excitação nm e força do oscilador da transição (OSC) obtidos para diferentes solventes e com dois funcionais distintos: CAM-B3LYP e B3LYP e o conjunto de funções base $6-311+\mathrm{G}^{* *}$. Forma $\mathrm{MBH}^{+}$. . . . . 89

5.6 Valores para energia de transição $\left(\mathrm{em}^{\mathrm{cm}}{ }^{-1}\right)$, comprimento de onda da excitação nm e força do oscilador da transição (OSC) obtidos para diferentes solventes e com dois funcionais distintos: CAM-B3LYP e B3LYP e o conjunto de funções base $6-311+\mathrm{G}^{* *}$. Forma MB. . . . . . 90

5.7 Valores experimentais para o comprimento de onda de máxima absorção, $\lambda_{\max }$, das formas $\mathrm{MB}$ e $\mathrm{MBH}^{+}$. O erro experimental é de $1 \mathrm{~nm}$.

5.8 Número de moléculas de água distribuidos ao redor da sonda, obtidos pela integração da função de mínima distância, MDDF. . . . . . . . . . 96

5.9 Valores para o comprimento de onda (em nm) da primeira trasnsição eletrônica obtidos para diferentes modelos de solvatação em água. O cálculo foi realizado com o método TD-DFT, funcionais CAM-B3LYP e B3LYP com o conjunto de funções base 6-311+G**. Em parênteses está apresentado o valor para a força do oscilador da respectiva transição

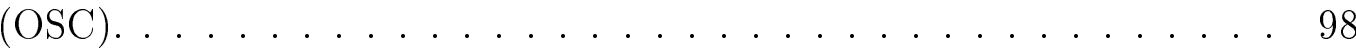

5.10 Número de moléculas de metanol distribuidos ao redor da sonda, obtidos pela integração da função de mínima distância, MDDF. . . . . . . . . . 102 
5.11 Valores para o comprimento de onda da excitação eletrônica (em nm) da primeira transição, obtidos para diferentes modelos de solvatação em metanol. O cálculo foi realizado com o método TD-DFT, funcionais CAM-B3LYP e B3LYP com o conjunto de funções base $6-311+\mathrm{G}^{* *}$. Em parênteses está apresentado o valor para a força do oscilador da respectiva transição (OSC). . . . . . . . . . . . . . . . 103

5.12 Número de moléculas de acetonitrila obtido até o final de cada camada de solvatação. . . . . . . . . . . . . . . . . . 107

5.13 Valores para o comprimento de onda da excitação eletrônica (em nm) da primeira transição, obtidos para diferentes modelos de solvatação em acetonitrila. O cálculo foi realizado com o método TD-DFT, funcionais CAM-B3LYP e B3LYP com o conjunto de funções base $6-311+\mathrm{G}^{* *}$. Em parênteses está apresentado o valor para a força do oscilador da respectiva transição (OSC). . . . . . . . . . . . . . . . 108

5.14 Valores para energia de transição $\left(\mathrm{em}^{-1}\right)$, comprimento de onda (em $\mathrm{nm}$ ) e força do oscilador (OSC) obtidos para o dímero $\mathrm{MB}_{2}$. Valores teóricos obtidos com TD-DFT usando os funcionais CAM-B3LYP e B3LYP e as funções base $6-311+\mathrm{G}^{* *}$. . . . . . . . . . . . . . . . 110

5.15 Valores para energia de transição $\left(\mathrm{em} \mathrm{cm}^{-1}\right)$, comprimento de onda (em $\mathrm{nm})$ entre colchetes e força do oscilador da transição entre parênteses obtidos para o dímero $\mathrm{MB}_{2}$ para diferentes modelos de solvatação. Valores teóricos obtidos com TD-DFT usando os funcionais CAM-B3LYP e B3LYP e as funções base $6-311+\mathrm{G}^{* *}$. . . . . . . . . . . . . . 115

5.16 Energia livre de fase gasosa para os constintuintes da reação ácido-base com a água em unidades atômica (u.a) e variação da energia livre, $\Delta G_{g}$, $\mathrm{em}(\mathrm{kcal} / \mathrm{mol}) . \ldots \ldots \ldots$. . . . . . . . . . . . . 119 
5.17 Energia livre de solvatação para os constintuintes da reação ácido-base com a água. . . . . . . . . . . . . . . . . . . . . 121

5.18 Contribuições para o cálculo do $\mathrm{pK}_{a}$. . . . . . . . . . . . . 121

5.19 Energia livre de fase gasosa para os constintuintes da reação ácidobase com o metanol em unidades atômica (u.a) e variação da energia livre $, \Delta G_{g}, \mathrm{em}(\mathrm{kcal} / \mathrm{mol})$.

5.20 Energia livre de solvatação para os constintuintes da reação ácido-base em metanol. . . . . . . . . . . . . . . . . . . . . . . 123

5.21 Contribuições para o cálculo do $\mathrm{pK}_{a}$. . . . . . . . . . . . . . 124

6.1 Valores de energia $(\mathrm{kcal} / \mathrm{mol})$, momento de dipolo (D), carga atômica (e), comprimento da ligação $(\AA)$ e ângulos diedros $\left(^{\circ}\right)$ para as sondas sol\begin{tabular}{|c|}
\hline vatocrômicas otimizadas na conformação trans com B3LYP $/ 6-311+\mathrm{G}^{* *}$ \\
\hline
\end{tabular} e dipolo com MP2 $/ 6-311+\mathrm{G}^{* *}$.

6.2 Valores de energia $(\mathrm{kcal} / \mathrm{mol})$, momento de dipolo (D), carga atômica (e), comprimento da ligação $(\AA)$ e ângulos diedros $\left(^{\circ}\right)$ para as sondas solvatocrômicas estudadas isoladamente na conformação cis. Geometria otimizada no nível B3LYP /6-311+G** e momento de dipolo obtido com MP2 $/ 6-311+\mathrm{G}^{* *} . \ldots \ldots \ldots \ldots \ldots$

6.3 Valores de energia $(\mathrm{kcal} / \mathrm{mol})$, momento de dipolo (D), carga atômica(e), \begin{tabular}{|c|}
\hline comprimento da ligação $(\AA)$ e ângulos diedros $\left(^{\circ}\right)$ para as sondas sol- \\
\hline vatocrômicas na conformação trans estudadas na forma zwiteriônica \\
\hline (ZW). Geometria otimizada no nível B3LYP $/ 6-311+\mathrm{G}^{* *}$ e momento de \\
\hline
\end{tabular} dipolo obtido com MP2 $/ 6-311+\mathrm{G}^{* *}$. 
6.4 Valores de energia $(\mathrm{kcal} / \mathrm{mol})$, momento de dipolo (D), carga atômica (e) e comprimento da ligação $(\AA)$ para as sondas na geometria protonada na conformação trans. Geometria otimizada no nível B3LYP $/ 6-311+\mathrm{G}^{* *}$ e momento de dipolo obtido com MP2/6-311+G**. . . . . . . . . . 134

6.5 Valores para energia da transição $\pi-\pi^{*}\left(\mathrm{~cm}^{-1}\right)$ e comprimento de onda $(\mathrm{nm})$ para as sondas MB e MePMBr${ }_{2}$ obtidos utilizando diferentes métodos de cálculos quânticos. . . . . . . . . . . . . . . . . . . 137

6.6 Valores para energia da transição $\pi-\pi^{*}\left(\mathrm{~cm}^{-1}\right)$ para as sondas estudadas obtidos utilizando as diferentes estruturas otimizadas para o estado fundamental da molécula. Entre parênteses apresentamos o valor para o - comprimento de onda de máxima absorção $(\mathrm{nm})$. . . . . . . . . . 138

6.7 Valores para energia da transição $\pi-\pi^{*}, \mathrm{E}\left(\mathrm{cm}^{-1}\right)$, e comprimento de onda, $\lambda_{\max }(\mathrm{nm})$, para as sondas estudadas na conformação trans. Valores teóricos obtidos com TD-DFT usando o funcional B3LYP e as

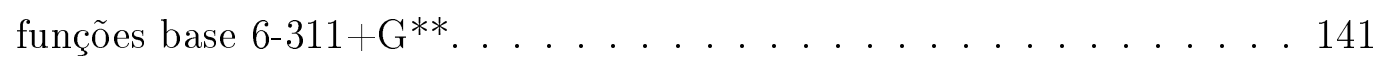

6.8 Valores para energia da transição $\pi-\pi^{*}, \mathrm{E}\left(\mathrm{cm}^{-1}\right)$, e comprimento de onda, $\lambda_{\max }(\mathrm{nm})$, para as sondas estudadas na conformação cis. Valores teóricos obtidos com TD-DFT usando o funcional B3LYP e as funções base $6-311+\mathrm{G}^{* *} . \ldots \ldots \ldots \ldots \ldots \ldots \ldots$

6.9 Valores para energia da transição $\pi-\pi^{*}, \mathrm{E}\left(\mathrm{cm}^{-1}\right)$, e comprimento de \begin{tabular}{|c|}
\hline onda, $\lambda_{\max }(\mathrm{nm})$, para as sondas estudadas na forma protonada. Valores \\
\hline
\end{tabular} teóricos obtidos com TD-DFT usando o funcional B3LYP e as funções

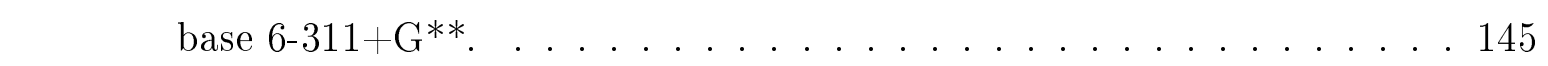


6.10 Valores para energia da transição $\pi-\pi^{*}, \mathrm{E}\left(\mathrm{cm}^{-1}\right)$, e comprimento de onda, $\lambda_{\max }(\mathrm{nm})$, para as sondas estudadas na forma zwiteriônica. Valores teóricos obtidos com TD-DFT usando o funcional B3LYP e as

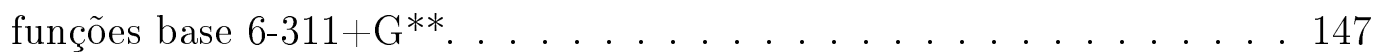

6.11 Efeito do solvente apenas na geometria para o espectro de absorção. Valores para energia da transição $\pi-\pi^{*}, \mathrm{E}\left(\mathrm{cm}^{-1}\right)$, e comprimento de onda, $\lambda_{\max }(\mathrm{nm})$ para as sondas estudadas na conformação trans. Va\begin{tabular}{|l|}
\hline lores teóricos obtidos com TD-DFT usando dois funcionais distintos, \\
\hline
\end{tabular} B3LYP e [CAM-B3LYP], e as funções base 6-311+ $\mathrm{G}^{* *}$. Geometria relaxada no solvente e espectro calculado em vácuo. . . . . . . . . . . . . 151

6.12 Efeito do solvente na geometria e no espectro de absorção. Valores para energia da transição $\pi-\pi^{*}, \mathrm{E}\left(\mathrm{cm}^{-1}\right)$, e comprimento de onda, $\lambda_{\max }$ (nm) para as sondas estudadas na conformação trans. Valores teóricos obtidos com TD-DFT usando dois funcionais distintos, B3LYP e [CAMB3LYP|, e as funções base 6-311+ $\mathrm{G}^{* *}$. Geometria relaxada no solvente e espectro calculado no respectivo solvente. . . . . . . . . . . . . . . 153

6.13 Número de moléculas do solvente 1.4 dioxano obtido até o final da camada de solvatação. Entre parênteses apresentamos os valores obtidos para o solvente DMSO. . . . . . . . . . . . . . . 160

6.14 Número de moléculas do solvente metanol obtido até o final da camada de solvatação. Entre parênteses apresentamos os valores obtidos para o solvente água. . . . . . . . . . . . . . . . . . . . 163

6.15 Valores para energia da transição $\pi-\pi^{*}\left(\mathrm{~cm}^{-1}\right)$ para as sondas estudadas. Valores teóricos obtidos com TD-DFT usando os funcional CAM-B3LYP|B3LYP e as funções base 6-311+G**. Realizado na configuração ASEC para o respectivo solvente. . . . . . . . . . . . . . . . . 164 
6.16 Valores para o comprimento da ligação $(\AA)$ e angulos diedros $\left(^{\circ}\right)$ para os monômeros obtidos da formação do dímero. Geometria do dímero otimizada no nível B3LYP /6-311+G** embebida no solvente 1,4 dioxano com PCM. . . . . . . . . . . . . . . . . . 167

6.17 Valores para energia da transição $\pi-\pi^{*}, \mathrm{E}\left(\mathrm{cm}^{-1}\right)$, e comprimento de onda, $\lambda_{\max }(\mathrm{nm})$, para os dímeros isolados. Valores teóricos obtidos com TD-DFT usando os funcional B3LYP e as funções base $6-311+\mathrm{G}^{* *}$ calculados em 1,4 dioxano usando PCM. . . . . . . . . . . . . . . 168

6.18 Valores para energia da transição $\pi-\pi^{*}\left(\mathrm{~cm}^{-1}\right)$ para os monômeros isolados. Valores teóricos obtidos com TD-DFT usando os funcional B3LYP (CAM-B3LYP) e as funções base $6-311+\mathrm{G}^{* *} . . . . .171$

6.19 Valores para energia da transição $\pi-\pi^{*}, \mathrm{E}\left(\mathrm{cm}^{-1}\right)$, e comprimento de onda, $\lambda_{\max }(\mathrm{nm})$, para os dímeros em 1,4 dioxano descrito como cargas pontuais. Valores teóricos obtidos com TD-DFT usando os funcional B3LYP e as funções base $6-311+\mathrm{G}^{* *}$. . . . . . . . . . . . . . 174

7.1 Valores para o comprimento de onda de máxima absorção, $\lambda_{\max }$, da primeira e segunda bandas de absorção obtidos da curva apresentada na

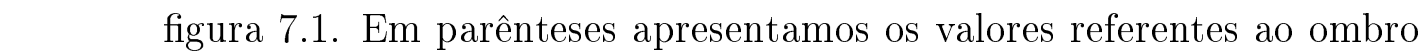
da primeira banda no solvente clorofórmio. O erro experimental é de 1 nm. . . . . . . . . . . . . . . . 177

7.2 Comparativo entre o comprimento de onda de máxima absorção, $\lambda_{\max }$ (nm), entre a banda da solução ácida e a segunda banda observada na solução alcalina. . . . . . . . . . . . . . . . . . . . . . 202 
A.1 Tabela com os parâmetros necessários para realizar uma simulação no DICE. Sonda MB, geometria e cargas obtidas na presença do solvente através do modelo contínuo PCM, solvente água. . . . . . . . . . . . . . 226

A.2 Tabela com os parâmetros necessários para realizar uma simulação no

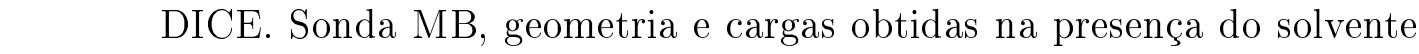
através do modelo contínuo PCM, solvente metanol. . . . . . . . . . . . 228

A.3 Tabela com os parâmetros necessários para realizar uma simulação no DICE. Sonda MB, geometria e cargas obtidas na presença do solvente através do modelo contínuo PCM, solvente acetonitrila. . . . . . . . . . 230

A.4 Tabela com os parâmetros necessários para realizar uma simulação no DICE. Sonda $\mathrm{MBH}^{+}$, geometria e cargas obtidas na presença do solvente através do modelo contínuo PCM, solvente água. . . . . . . . . . . . . . 232

A.5 Tabela com os parâmetros necessários para realizar uma simulação no DICE. Sonda $\mathrm{MBH}^{+}$, geometria e cargas obtidas na presença do solvente através do modelo contínuo PCM, solvente metanol. . . . . . . . . . . . 235

A.6 Tabela com os parâmetros necessários para realizar uma simulação no \begin{tabular}{|c|}
\hline DICE. Sonda $\mathrm{MBH}^{+}$, geometria e cargas obtidas na presença do solvente \\
\hline através do modelo contínuo PCM, solvente acetonitrila. . . . . . . . . 237
\end{tabular}

A.7 $\quad$ Parâmetros obtidos do modelo SPC para a água |105|. . . . . . . . . . 239

A.8 Parâmetros obtidos para o solvente metanol |107|. . . . . . . . . . 239

A.9 $\quad$ Parâmetros obtidos para o solvente acetonitrila |108|. _ . . . . . . . 240 


\section{Capítulo 1}

\section{Introdução}

Um dos objetivos atuais da área de física molecular teórica está em conseguir uma boa compreensão e predição dos fenômenos ocorridos em solução com o mesmo êxito obtido para o tratamento de propriedades eletrônicas de moléculas em fase gasosa. A dificuldade nessa tarefa se concentra, em boa parte, no grande número de configurações acessíveis à um sistema líquido em determinadas condições termodinâmicas, o que se faz necessária a inclusão de um número considerável de configurações para os cálculos quânticos para que tenhamos uma grandeza representativa do ponto de vista estatístico. Soma-se a isso a necessidade de inclusão de um grande número de moléculas do sistema para obtermos uma boa descrição dos efeitos de longo alcance. Essas duas condições, necessárias para uma boa descrição de um sistema solvatado, fazem com que o tratamento teórico de propriedades eletrônicas de sistemas líquidos seja muito dispendioso do ponto de vista computacional.

Um dos interesses em estudar sistemas em fase líquida reside no fato de que o solvente pode modificar as propriedades de um soluto de várias formas. Ele pode, em particular, afetar a estabilidade conformacional de moléculas $[1-3 \mid$. Isso acontece porque, em geral, a diferença de energia livre favorece uma conformação em um dado solvente e outra conformação num solvente com polaridade diferentes. O que se tem como senso comum é que a conformação com maior momento de dipolo deve ser mais estável 
em solventes com maior polaridade [4]. Adicionalmente o meio solvente pode exercer influência sobre várias propriedades eletrônicas como, por exemplo, a posição e intensidade de bandas espectrais [5-10]. Na literatura é sabido que o espectro eletrônico de absorção de um soluto medido em solventes de diferentes polaridades pode apresentar mudanças na posição, intensidade e na forma das bandas de absorção [6,7, 10]. O termo solvatocromismo é usado para descrever as mudanças na posição dos comprimentos de onda de máxima absorção, $\lambda_{\max }$, devido à mudança na polaridade do meio. Define-se como um solvatocromismo negativo (deslocamento hipsocrômico ou deslocamento para o azul) o deslocamento do $\lambda_{\max }$ para menores comprimentos de onda com o aumento da polaridade do solvente. O solvatocromismo positivo (deslocamento batocrômico ou deslocamento para o vermelho) é definido por um deslocamento do $\lambda_{\max }$ para maiores comprimentos de onda quando a polaridade do solvente é aumentada.

Na descrição do solvatocromismo, as propriedades físicas e químicas do solvente são muito importantes para uma completa compreensão a respeito da interação solutosolvente. O efeito dessa interação pode ser discutido em função da polaridade do solvente. Entende-se como polaridade do solvente a sua capacidade de interação e modificação do soluto, onde estão inclusas todas as interações intermoleculares específicas e não específicas. Portanto, polaridade é a soma de todas as propriedades moleculares responsáveis por todas as forças de interação (Coulomb, direcional, indutiva, dispersão, ligações de hidrogênio e forças de interação envolvendo doador e receptor de pares de elétrons) 8 .

A polaridade do solvente tem sido comumente estudada através da energia de transição eletrônica $\left(E_{T}\right)$ de corantes solvatocrômicos. Esses corantes são moléculas que apresentam bandas de absorção na região do ultravioleta e visível (UV-vis) do espectro e sofrem deslocamentos à medida que a polaridade do solvente se altera [9]. Com isso pode-se obter parâmetros empíricos de polaridade dos solventes através de medidas do espectro UV-vis. Por isso, foram realizados estudos do espectro de absorção 
de uma grande variedade de compostos, que apresentam dependência com a polaridade do solvente, no intuito de encontrar uma possível referência para uma escala empírica de polaridade que possibilite relacionar de forma segura as propriedades físico-químicas apresentada pelos sistemas estudados com a natureza do solvente. Isso fez com que inúmeras escalas de polaridades fossem sugeridas $10,12,14$.

Uma escala de polaridade obtida através de medidas espectróscopicas na região UV-vis, é a escala $E_{T}(30)$ [10,11. Essa é uma escala uniparamétrica de polaridade dos solventes e se baseia no forte solvatocromismo exibido pelo corante 2,6-difenil-4(2,4,6trifenil-1-piridínio)-1-fenolato, conhecido como betaína 30 de Reichardt, e é definida pela energia de transição eletrônica $(\mathrm{kcal} / \mathrm{mol})$ desse corante. Essa energia é obtida através da equação

$$
E_{T}(\operatorname{sonda})(\mathrm{kcal} / \mathrm{mol})=28591.5 / \lambda_{\max }(\mathrm{nm})
$$

onde $\lambda_{\max }$ é o valor do comprimento de onda do máximo de absorção da banda de transferência de carga do corante. Essa equação converte o comprimento de onda da transição eletrônica ocorrida na sonda em energia correspondente à transferência de cargas. Assim, a partir de medidas do espectro dessa sonda em vários solventes, Reichardt [11 construiu uma escala de polaridade do solvente de maneira empírica. A betaína 30 de Reichardt foi escolhida como uma sonda de referência por ser solúvel em vários solventes, desde polares a apolares. Seu espectro de absorção sofre um forte solvatocromismo negativo, possuindo um $\lambda_{\max }$ de $931 \mathrm{~nm}$ em tetrametilsilano (TMS) e $453 \mathrm{~nm}$ em água. Esta sonda também não apresenta mudança conformacional, agregação, tautomerismo ou qualquer mudança em sua estrutura que dê origem ao solvatocromismo. Sendo assim, o deslocamento do $\lambda_{\max }$ é devido apenas às interações soluto-solvente, não sendo provocada por uma mudança interna na geometria do soluto ou por interações soluto-soluto. Desta forma, comparando a energia de transição $E_{T}(30)$ nos vários solventes com a energia de transição de outras sondas, $E_{T}$ (sonda), 
pode-se tentar extrair informações sobre a natureza dos deslocamentos solvatocrômicos dessas outras moléculas.

A polaridade do solvente também pode ser definida considerando o parâmetro $E_{T}$ normalizado. A normalização é feita usando como referência os solventes tetrametilsilano $(\mathrm{TMS})\left(E_{T}^{N}=0\right)$ e água $\left(\mathrm{H}_{2} \mathrm{O}\right)\left(E_{T}^{N}=1\right)$. O parâmetro $E_{T}^{N}$ pode ser calculado a partir de $E_{T}(30)$ pela equação

$$
E_{T}^{N}=\frac{E_{T}(30)-E_{T}(30)_{T M S}}{E_{T}(30)_{H_{2} O}-E_{T}(30)_{T M S}}=\frac{E_{T}(30)-30.7}{32.4}
$$

Um fenômeno observado experimentalmente há pouco mais de 30 anos [15], no estudo do espectro de absorção de moléculas em solventes polares e apolares, mostra uma inversão no comportamento solvatocrômico de algumas espécies dependente da polaridade do meio. Esse fenômeno é chamado de solvatocromismo reverso e foi objeto de nosso estudo neste trabalho.

Atualmente há algumas hipóteses para a interpretação do solvatocromismo reverso, que se baseiam em mudanças conformacionais do soluto ou agregação. Sendo assim, o solvatocromismo reverso não teria origem apenas nas interações soluto-solvente, mas também devido à mudanças no soluto e interações soluto-soluto. Essas hipóteses são:

(i) Mudança na estrutura do estado fundamental da molécula entre as formas mesoméricas quinoidal (neutra)/ benzoidal (zwiteriônica). A maior contribuição de uma ou outra forma mesomérica depende da estrutura da sonda e também da polaridade do solvente 16 ;

(ii) Mudança na estrutura do estado fundamental da molécula entre as formas rotaméricas cis/trans (syn/anti nomeclatura mais moderna) dependente da polaridade do meio 17 ; 
(iii) Formação de dímeros ou agregados dependente da polaridade do solvente [18, 19 .

Com o interesse de investigar a validade dessas hipóteses para o comportamento do solvatocromismo reverso, nós iremos estudar 4 sondas solvatocrômicas, que são moléculas cujos espectros de absorção são bastante sensíveis ao solvente e pertencem a família das merocianinas [20]. Essa família de compostos apresenta um grupo doador de elétrons (D) ligado por um sistema conjugado (R) e a um grupo aceitador de elétrons (A). Portanto, sua estrutura pode existir na forma neutra (N), zwiteriônica (Z) ou híbrida devido a ressonância entre $\mathrm{N}$ e $\mathrm{Z}, D-R-A \rightleftarrows D^{+}-R-A^{-}$. A transição eletrônica das merocianinas está associada a uma transferência parcial de carga intramolecular entre o grupo doador de elétrons e o receptor, que depende muito da polaridade do meio, é isso que provoca grandes deslocamentos no comprimento de onda máximo das bandas de absorção das merocianinas.

Neste trabalho estudaremos a Merocianina de Brooker (4-[1-metil-4(1H)-piridinilidenoetilideno]-2,5-cicloexadien-1-ona) (MB) [21], figura 1.1 e alguns de seus derivados (figura 1.3).

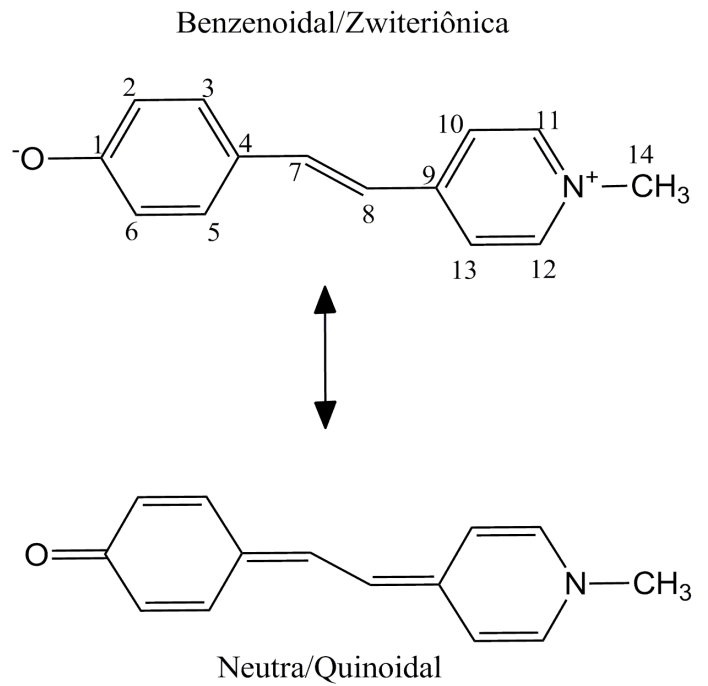

Figura 1.1: Ilustração da Merocianina de Brooker (MB).

A MB possui um comprimento de onda de máxima absorção, $\lambda_{\max }$, de $620 \mathrm{~nm}$ 
$\left(16142 \mathrm{~cm}^{-1}\right)$ em clorofórmio e $442 \mathrm{~nm}\left(22624 \mathrm{~cm}^{-1}\right)$ em água, apresentando um forte deslocamento com a mudança de polaridade do meio [22]. Esse comportamento está apresentado na figura 1.2 .

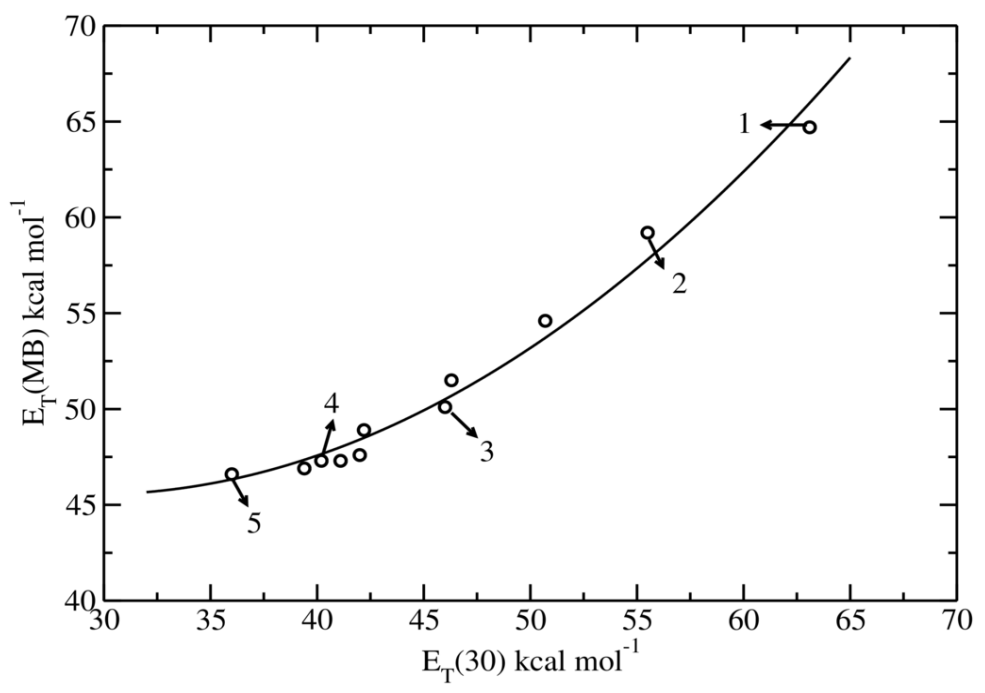

Figura 1.2: Correlação entre $E_{T}(\mathrm{MB})$ e $E_{T}(30)$. Dados obtidos da referência [22]. Os solventes que estão numerados nos gráficos são: água (1), metanol (2), acetonitrila (3), piridina (4) e dioxano (5). A medida em dioxano foi obtida pela extrapolação de misturas binárias de solventes.

Os dados experimentais foram obtidos da referência [22]. Note que $E_{T}(\mathrm{MB})$ apresenta uma relação não linear com $E_{T}(30)$. Esse comportamento é considerado anômolo e despertou a atenção de muitos pesquisadores teóricos 23 26 experimentais 22, 25, 28, 29]. Entretanto, ainda existem várias questões em aberto para a compreensão do efeito de solventes no espectro de absorção da MB. Por isso, utilizamos a MB e 3 derivados bromatados (figura 1.3) como objeto de estudo neste trabalho. No caso da MB, além de realizarmos os estudos teóricos através de cálculos quânticos e simulações computacionais em solvente, também realizamos medidas experimentais do espectro de absorção da sonda para diferentes solventes, variando o pH da solução e variando a concentração do soluto. Essas medidas foram necessárias por não estarem disponivéis na literatura e serão apresentadas no capítulo de resultados.

As moléculas derivadas da MB (figura 1.3) que estudamos neste trabalho, foram 
previamente estudadas através de medidas experimentais pelo grupo do prof. Omar El Soud do Departamento de Química Fundamental-Instituto de Química-USP (IQ-USP) e foi observado um comportamento distinto para o solvatocromismo de cada uma das três sondas $[30$.<smiles>CN1C=CC(=CC=C2C=C(Br)C(=O)C(Br)=C2)C=C1</smiles>

(a) $\mathrm{MePMBr}_{2}$<smiles>CN1C=C/C(=C\C=C2C=C(Br)C(=O)C(Br)=C2)c2ccccc21</smiles>

(b) $\mathrm{MeQMBr}_{2}$<smiles>CN1c2ccccc2C(=CC=C2C=C(Br)C(=O)C(Br)=C2)c2ccccc21</smiles>

(c) $\mathrm{MeAMBr}_{2}$

Figura 1.3: Derivados da MB que foram estudadas neste trabalho. (a) 2,6dibromo-4-[(E)-2-(1 methylpyridinium-4yl)ethenyl]phenolate( $\left.\mathrm{MePMBr}_{2}\right)$; (b)2,6-dibromo-4[(E)-2(1-methylquinolinium-4-yl)ethenyl]phenolate $\left(\mathrm{MeQMBr}_{2}\right)$; (c) 2,6-dibromo-4-[(E)-2-(1methylacridinium-4-yl)ethenyl]phenolate $\left(\mathrm{MeAMBr}_{2}\right)$.

As sondas derivadas da $\mathrm{MB}\left(\mathrm{MePMBr}_{2}, M e Q M B r_{2}\right.$ e $M e A M B r_{2}$, apresentadas na figura 1.3 diferem entre si pela presença de 1 ou 2 anéis aromáticos, além do anel heterocíclico, esse processo é conhecido como anelação. Experimentalmente, foi obtido o espectro de absorção das sondas em 38 solventes próticos e apróticos [30, e foram observadas correlações não lineares entre as escalas empíricas de polaridade, $E_{T}\left(\right.$ sonda) e $E_{T}(30)$. De maneira resumida observou-se que a escala $E_{T}\left(M e P M B r_{2}\right)$ apresenta uma relação linear com a escala $E_{T}(30)$, já as escalas $E_{T}\left(M e Q M B r_{2}\right)$ e $E_{T}\left(M e A M B r_{2}\right)$ apresentam relações não lineares com a escala $E_{T}(30)$, ver figura 1.4 . A sonda $\mathrm{MePMBr}_{2}$ também foi investigada por cálculos teóricos [31], nesse trabalho foi estudado o efeito da flexibilidade e da polarização em água no seu espectro UV-vis. 

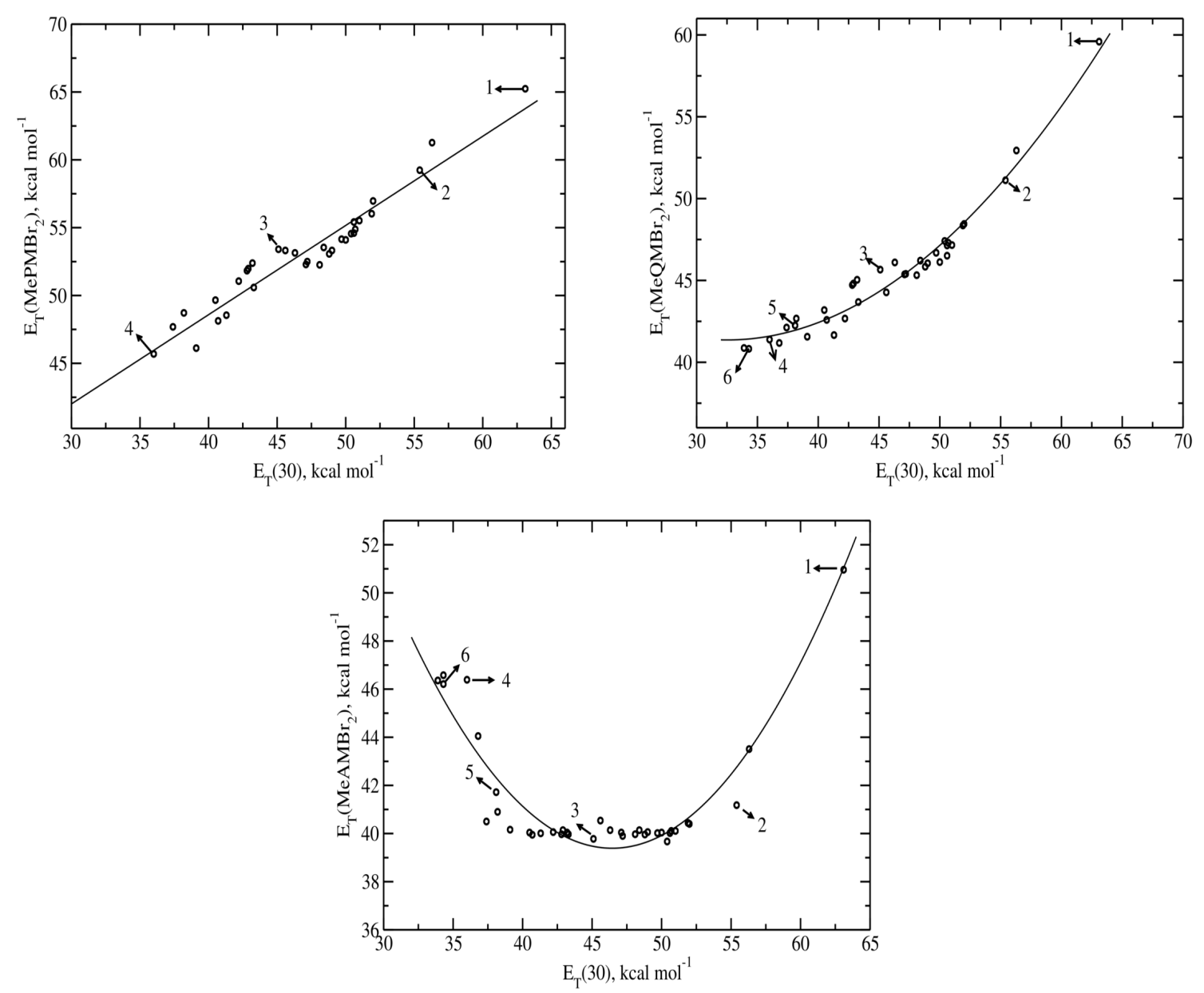

Figura 1.4: Correlação entre $E_{T}$ (sonda) e $E_{T}(30)$ para os 38 solventes estudados experimentalmente. Valores para $E_{T}$ foram obtidos da referência [30]. Os solventes que estão numerados nos gráficos são: água (1), metanol (2), DMSO (3), 1,4 dioxano (4), acetato de etila (5), e benzeno (6).

De acordo com a figura 1.4 o processo de anelação das sondas provoca o comportamento reverso para o solvatocromismo [30]. Desta forma, neste trabalho, estudamos quais são as mudanças nas interações soluto-solvente devido a mudança estrutural da sonda provocado pelo acréscimo de 1 ou 2 anéis aromáticos. 


\subsection{Revisão bibliográfica: Merocianina de Brooker}

Desde o trabalho de Brooker [21], um grande número de artigos tem sido publicado investigando o forte solvatocromismo sofrido pelas merocianinas $16,18,21,28,30$. A merocianina de Brooker pertence a família das merocianinas e é uma das moléculas que tem sido bastante estudada ao longo do tempo tanto por trabalhos experimentais quanto por abordagens teóricas $[21,27,29,32,37]$. O interesse no estudo desse sistema se dá pela presença de algumas propriedades particulares dessa molécula:

(i) Apresenta um grande deslocamento solvatocrômico devido à mudança na polaridade do meio. Por exemplo, ela possui um comprimento de onda de máxima absorção em clorofórmio de $620 \mathrm{~nm}\left(16142 \mathrm{~cm}^{-1}\right)$ e uma máxima absorção em $442 \mathrm{~nm}\left(22624 \mathrm{~cm}^{-1}\right)$ em água 22 . Isso corresponde a um deslocamento para o azul de $6393 \mathrm{~cm}^{-1}$.

(ii) Apresenta uma correlação não linear do solvatocromismo com a escala de polaridade $E_{T}(30)$, ver figura 1.2 . Esse comportamento é considerado anômolo e por isso muitos pesquisadores tentam descobrir a origem desse comportamento.

(iii) Podemos destacar também a sua alta hiperpolarizabilidade que resulta em propriedades não-lineares que são tecnicamente importantes [38.

O solvatocromismo experimental da MB tem sido caracterizado por um forte deslocamento hipsocrômico, acompanhado de uma redução do coeficiente de extinção molar, $\varepsilon_{\max }$, a medida que a polaridade do meio é aumentada [22]. A explicação desses dois comportamentos tem sido buscada considerando-se duas estruturas, a forma zwiteriônica e a forma neutra (ver figura 1.1 21, 22, 25, 29]. Segundo essa hipótese, haveria uma maior população da forma zwiteriônica (polar) em solventes polares, e por outro lado, a forma neutra seria predominante em solventes apolares. 
A abordagem teórica do forte solvatocromismo das merocianinas tem sido investigado por métodos teóricos que utilizam cálculos de mecânica quântica acoplados com diferentes modelos para o solvente $[22-26]$. Os modelos comumente usados para o solvente são: o modelo contínuo polarizável (PCM) e os métodos de mecânica quântica/mecânica molecular (QM/MM). Um ponto em comum nesses trabalhos é a descrição da tendência do deslocamento da primeira banda do espectro espectro UV-vis observado experimentalmente para maiores energias (deslocamento para o azul) quando há um aumento na polaridade do solvente bem como a descrição correta do valor absoluto do $\lambda_{\max }$ em solventes de alta polaridade. No entanto, o valor absoluto de $\lambda_{\max }$ em baixa polaridade é subestimado, e isso ocorre independente do método de cálculo quântico utilizado e também independente do modelo considerado para o solvente. Como exemplo, mostramos os valores calculados por Murugan e colaboradores [23, 24], obtiveram um valor de $\lambda_{\max }=441 \mathrm{~nm}$ para a absorção em água e $\lambda_{\max }=485 \mathrm{~nm}$ em clorofórmio. Notemos que existe uma excelente concordância para o valor de $\lambda_{\max }$ em água (valor exp. de $442 \mathrm{~nm}\left[22\right.$ ), porém o valor para $\lambda_{\max }$ em clorofórmio (valor exp. de 620 nm [22]) está em desacordo por $135 \mathrm{~nm}\left(4489 \mathrm{~cm}^{-1}\right)$. Essa discrepância entre os dados experimentais e teóricos para solvente de baixa polaridade também foi tema de estudo neste trabalho.

\subsection{Organização da Tese}

A exposição dos demais capítulos desta tese está dividida 8 capítulos que podemos agrupar em três partes:

Na primeira parte apresentamos as metodologias utilizadas. No capítulo 2 apresentamos os métodos utilizados para o estudo da estrutura eletrônica do soluto. No capítulo 3 descrevemos as metodologias utilizadas para a inclusão do solvente e no capítulo 4 será descrito os materiais e métodos utilizados para as medidas experimen- 
tais.

Na segunda parte apresentamos e discutimos os resultados obtidos. Nos capítulos 5 e 6 apresentamos os resultados teóricos obtidos para a Merocianina de Brooker e alguns derivados. Nos capítulos 7 apresentamos os resultados experimentais obtidos para a Merocianina de Brooker.

Na terceira e última parte, capítulo 8, apresentamos as conclusões deste trabalho.

Em toda a tese nós utilizamos a notação inglesa para casa decimal. Separador com ponto (.). 


\section{Capítulo 2}

\section{Métodos quânticos para representação do soluto.}

\subsection{Introdução}

Neste capítulo descrevemos os métodos de estrutura eletrônica que foram utilizados na realização deste trabalho. Abordamos de forma sucinta o método Hartree-Fock e métodos perturbativos pós Hartree-Fock. Falaremos também sobre a Teoria do Funcional da Densidade.

O objetivo central, utilizando métodos quânticos, é a obtenção de soluções da equação de Schrödinger para a determinação de propriedades de sistemas atômicos e moleculares. Porém, esse é um problema extremamente complicado e os diferentes métodos para a descrição da estrutura eletrônica de átomos e moléculas refletem as várias propostas de resolução dessa equação.

A equação de Schrödinger independente do tempo para uma molécula composta por N elétrons e M núcleos é escrita como:

$$
\mathcal{H}(\mathbf{r}, \mathbf{R}) \Psi(\mathbf{r}, \mathbf{R})=E \Psi(\mathbf{r}, \mathbf{R})
$$

onde $\mathcal{H}(\mathbf{r}, \mathbf{R})$ é o operador hamiltoniano, $\Psi(\mathbf{r}, \mathbf{R})$ é a função de onda total do sistema, 
$\mathbf{r}=\left(\mathbf{r}_{1}, \mathbf{r}_{2}, \ldots, \mathbf{r}_{\mathbf{N}}\right)$ e $\mathbf{R}=\left(\mathbf{R}_{1}, \mathbf{R}_{\mathbf{2}}, \ldots, \mathbf{R}_{\mathbf{M}}\right)$ são as coordenadas dos elétrons e dos núcleos, respectivamente $[39]$. O hamiltoniano que descreve um sistema com $N$ elétrons e $M$ núcleos é expresso, em unidades atômicas (Hartree), por:

$$
\mathcal{H}(\mathbf{r}, \mathbf{R})=-\sum_{i=1}^{N} \frac{1}{2} \nabla_{i}^{2}-\sum_{A=1}^{M} \frac{1}{2 M_{A}} \nabla_{A}^{2}-\sum_{i=1}^{N} \sum_{A=1}^{M} \frac{Z_{A}}{r_{i A}}+\sum_{i<j}^{N} \frac{1}{r_{i j}}+\sum_{A<B}^{M} \frac{Z_{A} Z_{B}}{R_{A B}}
$$

onde os índices i e j referem-se aos elétrons e $\mathrm{A}$ e $\mathrm{B}$ aos núcleos, $\mathrm{Z}_{A}$ é a carga do núcleo A, $\mathrm{M}_{A}$ é a massa do núcleo A.

O primeiro passo para resolver a equação (2.1) é a separação dos movimentos nuclear e eletrônico. A aproximação mais usada é a aproximação de Born-Oppenheimer.

\subsection{Aproximação de Born-Oppenheimer}

A Aproximação de Born-Oppenheimer [40, 41] é fundamentada na diferença de escala energética que existe entre o núcleo atômico e o elétron. Sendo assim o hamiltoniano da equação $\left(2.2\right.$ pode ser separado em uma parte eletrônica $\left(\mathcal{H}^{\text {ele }}(\mathbf{r} ; \mathbf{R})\right)$ e outra nuclear $\left(\mathcal{H}^{N}(\mathbf{R})\right)$,

$$
\mathcal{H}(\mathbf{r}, \mathbf{R})=\mathcal{H}^{\text {ele }}(\mathbf{r} ; \mathbf{R})+\mathcal{H}^{N}(\mathbf{R})
$$

onde:

$$
\begin{gathered}
\mathcal{H}^{\text {ele }}(\mathbf{r} ; \mathbf{R})=-\sum_{i=1}^{N} \frac{1}{2} \nabla_{i}^{2}-\sum_{i=1}^{N} \sum_{A=1}^{M} \frac{Z_{A}}{r_{i A}}+\sum_{i<j}^{N} \frac{1}{r_{i j}} \\
\mathcal{H}^{N}(\mathbf{R})=-\sum_{A=1}^{M} \frac{1}{2 M_{A}} \nabla_{A}^{2}+\sum_{A<B}^{M} \frac{Z_{A} Z_{B}}{R_{A B}}
\end{gathered}
$$

Da mesma forma que o hamiltoniano total é obtido pela soma da parte eletrônica com a parte nuclear, a função de onda $\Psi(\mathbf{r}, \mathbf{R})$, que é autofunção de $\mathcal{H}(\mathbf{r}, \mathbf{R})$, pode 
ser escrita como o produto das funções de onda da parte eletrônica $\left(\Psi^{\text {ele }}(\mathbf{r} ; \mathbf{R})\right)$ com as autofunções do hamiltoniano nuclear $\left(\Psi^{N}(\mathbf{R})\right)$, logo:

$$
\Psi(\mathbf{r}, \mathbf{R}) \approx \Psi^{\text {ele }}(\mathbf{r} ; \mathbf{R}) \Psi^{N}(\mathbf{R})
$$

onde $\Psi^{\text {ele }}(\mathbf{r} ; \mathbf{R})$ depende apenas parametricamente de $\mathbf{R}$. Uma vez que $\mathcal{H}^{\text {ele }}(\mathbf{r} ; \mathbf{R})$ e $\mathbf{R}$ podem ser diagonalizados simultaneamente, os autovalores do hamiltoniano eletrônico podem ser determinados para valores fixos das posições nucleares $\mathbf{R}[39]$.

Reescrevendo a equação (2.1), temos:

$$
\left(\mathcal{H}^{\text {ele }}(\mathbf{r} ; \mathbf{R})+\mathcal{H}^{N}(\mathbf{R})\right) \Psi^{\text {ele }}(\mathbf{r} ; \mathbf{R}) \Psi^{N}(\mathbf{R})=E \Psi^{\text {ele }}(\mathbf{r} ; \mathbf{R}) \Psi^{N}(\mathbf{R})
$$

$$
\mathcal{H}^{\text {ele }}(\mathbf{r} ; \mathbf{R}) \Psi^{\text {ele }}(\mathbf{r} ; \mathbf{R}) \Psi^{N}(\mathbf{R})+\mathcal{H}^{N}(\mathbf{R}) \Psi^{\text {ele }}(\mathbf{r} ; \mathbf{R}) \Psi^{N}(\mathbf{R})=E \Psi^{\text {ele }}(\mathbf{r} ; \mathbf{R}) \Psi^{N}(\mathbf{R})
$$

onde o operador eletrônico atua somente na função de onda eletrônica e o operador nuclear atua na função de onda nuclear. Dividindo a expressão 2.8 por $\Psi^{\text {ele }}(\mathbf{r} ; \mathbf{R}) \Psi^{N}(\mathbf{R})$, obtemos:

$$
\begin{aligned}
& \frac{1}{\Psi^{\text {ele }}(\mathbf{r} ; \mathbf{R})} \mathcal{H}^{\text {ele }}(\mathbf{r} ; \mathbf{R}) \Psi^{\text {ele }}(\mathbf{r} ; \mathbf{R})+\frac{1}{\Psi^{N}(\mathbf{R})} \mathcal{H}^{N}(\mathbf{R}) \Psi^{N}(\mathbf{R})=E \\
& \frac{1}{\Psi^{\text {ele }}(\mathbf{r} ; \mathbf{R})} \mathcal{H}^{\text {ele }}(\mathbf{r} ; \mathbf{R}) \Psi^{\text {ele }}(\mathbf{r} ; \mathbf{R})=E-\frac{1}{\Psi^{N}(\mathbf{R})} \mathcal{H}^{N}(\mathbf{R}) \Psi^{N}(\mathbf{R})
\end{aligned}
$$

O termo à direita na igualdade da equação 2.10 depende somente das coordenadas nucleares, identificando esse termo com $\epsilon(\mathbf{R})$ temos:

$$
\mathcal{H}^{\text {ele }}(\mathbf{r} ; \mathbf{R}) \Psi^{\text {ele }}(\mathbf{r} ; \mathbf{R})=\epsilon(\mathbf{R}) \Psi^{\text {ele }}(\mathbf{r} ; \mathbf{R})
$$


onde

$$
\epsilon(\mathbf{R})=E-\frac{1}{\Psi^{N}(\mathbf{R})} \mathcal{H}^{N}(\mathbf{R}) \Psi^{N}(\mathbf{R})
$$

$\log \mathrm{O}$

$$
\mathcal{H}^{N}(\mathbf{R}) \Psi^{N}(\mathbf{R})+\epsilon(\mathbf{R}) \Psi^{N}(\mathbf{R})=E \Psi^{N}(\mathbf{R})
$$

que é a equação para o movimento dos núcleos, onde E é o autovalor da equação (2.1).

Portanto, a aproximação de Born-Oppenheimer separa a equação (2.1) em duas equações, 2.11 e (2.13), onde 2.11 é a equação do movimento eletrônico e 2.13 é a equação para o movimento dos núcleos. Devido à separação desses movimentos podemos fixar os núcleos em uma dada posição e então calcular $\Psi^{e l e}(\mathbf{r} ; \mathbf{R})$ pela equação (2.11). Assim, usando a equação (2.13) podemos obter a energia total, E(R). Contudo, vale lembrar que existem situações de forte acoplamento entre o movimento eletrônico e o movimento nuclear, onde a aproximação de Born-Oppenheimer não é válida [40].

Observando que para $\mathbf{R}$ fixo o único termo não nulo da expressão (2.5) é o potencial de repulsão núcleo-núcleo e que $\mathcal{H}^{\text {ele }}$ atua somente na função de onda eletrônica, podemos reescrever a equação (2.8) da seguinte forma:

$$
\epsilon(\mathbf{R}) \Psi^{\text {ele }}(\mathbf{r} ; \mathbf{R}) \Psi^{N}(\mathbf{R})+\sum_{A<B}^{M} \frac{Z_{A} Z_{B}}{R_{A B}} \Psi^{\text {ele }}(\mathbf{r} ; \mathbf{R}) \Psi^{N}(\mathbf{R})=E(\mathbf{R}) \Psi^{\text {ele }}(\mathbf{r} ; \mathbf{R}) \Psi^{N}(\mathbf{R})
$$

como $\mathbf{R}$ é mantido fixo, $\epsilon(\mathbf{R})$ e o potencial de repulsão núcleo-núcleo são números, então podemos dividir a equação 2.14 por $\Psi^{\text {ele }}(\mathbf{r} ; \mathbf{R}) \Psi^{N}(\mathbf{R})$,

$$
E(\mathbf{R})=\epsilon(\mathbf{R})+\sum_{A<B}^{M} \frac{Z_{A} Z_{B}}{R_{A B}}
$$

que é o potencial sentido pelos núcleos.

Portanto, resolvendo a equação 2.11 para $\mathbf{R}$, obtemos uma superfície de energia potencial $\mathrm{E}(\mathbf{R})$. Usando a equação 2.13 podemos encontrar a função de onda que descreve o movimento nuclear, $\Psi^{N}(\mathbf{R})$. 
Nas seções seguintes consideraremos apenas o movimento eletrônico dado pela equação (2.11), tornando desnecessário o uso de superescritos para distinguir os termos eletrônicos e nucleares.

\subsection{Método de Hartree-Fock}

A aproximação fundamental da teoria de Hartree-Fock (HF) é que cada elétron se move em um campo elétrico estático, criado por todos os outros elétrons. Fazendo uso dessa aproximação o método de Hartree-Fock busca uma solução aproximada para o estado fundamental de um sistema de elétrons. Essa teoria é a base para todos os outros métodos pós-HF, por isso a sua importância no estudo de sistemas multieletrônicos. O método teve início com a aproximação proposta por Hartree [42, 43 que consiste em considerar a função de onda de um sistema multieletrônico $(\Psi(\mathbf{r}))$ como o produto de funções de onda de um elétron $\left(\varphi_{i}\left(\mathbf{r}_{i}\right)\right)$, equação (2.16). Nessa aproximação, os elétrons de um sistema multieletrônico movem-se de maneira independente.

$$
\Psi(\mathbf{r})=\varphi_{1}\left(\mathbf{r}_{1}\right) \varphi_{2}\left(\mathbf{r}_{2}\right) \ldots \varphi_{N}\left(\mathbf{r}_{N}\right)
$$

As funções de onda de um elétron são chamadas de orbitais, sendo assim os $\varphi_{i}$ são orbitais espaciais e $\mathbf{r}_{i}$ representa as coordenadas espaciais do elétron $i$.

Ainda que a função de onda da equação (2.16) seja autofunção do hamiltoniano de um sistema multi-eletrônico, ela não representa um estado físico, pois não satisfaz ao princípio de exclusão de Pauli [45], que diz que a função de onda que descreve um sistema de muitos elétrons deve ser antissimétrica perante uma troca das coordenadas espaciais e de spin de dois elétrons quaisquer. Para resolver esse problema, em 1930, Fock [44] propôs uma modificação na função de onda multieletrônica proposta por Hartree, sugerindo o uso do determinante de Slater [46] para a construção de uma 
função de onda $\Psi_{0}$,

$$
\Psi_{0}=\frac{1}{\sqrt{N !}}\left|\begin{array}{cccc}
\chi_{1}\left(\mathbf{x}_{1}\right) & \chi_{2}\left(\mathbf{x}_{1}\right) & \ldots & \chi_{N}\left(\mathbf{x}_{1}\right) \\
\chi_{1}\left(\mathbf{x}_{2}\right) & \chi_{2}\left(\mathbf{x}_{2}\right) & \ldots & \chi_{N}\left(\mathbf{x}_{2}\right) \\
\vdots & \vdots & & \vdots \\
\chi_{1}\left(\mathbf{x}_{N}\right) & \chi_{2}\left(\mathbf{x}_{N}\right) & \ldots & \chi_{N}\left(\mathbf{x}_{N}\right)
\end{array}\right|
$$

sendo $N$ o número total de elétrons e os $\chi^{\prime} s$ funções dependentes das coordenadas espaciais e de spin de um único elétron, chamadas spin-orbitais moleculares [47]. Admitindo que os $\chi^{\prime} s$ são ortonormais, o fator $\frac{1}{\sqrt{N !}}$ é uma constante de normalização para $\Psi_{0}$. Uma notação mais compacta é normalmente encontrada,

$$
\begin{gathered}
\Psi_{0}=\frac{1}{\sqrt{N !}} \operatorname{det}\left(\chi_{1} \chi_{2} \ldots \chi_{N}\right) \\
\Psi_{0}=\left|\chi_{1} \chi_{2} \ldots \chi_{N}\right\rangle
\end{gathered}
$$

Em princípio, as dependências com relação às partes espacial e de spin dos spinorbitais moleculares podem ser separadas,

$$
\chi_{i}\left(\mathbf{x}_{1}\right)=\varphi_{i}\left(\mathbf{r}_{1}\right) \alpha(1)
$$

$\mathrm{Ou}$

$$
\chi_{i}\left(\mathbf{x}_{1}\right)=\varphi_{i}\left(\mathbf{r}_{1}\right) \beta(1)
$$

onde $\alpha$ e $\beta$ representam, respectivamente, spin para cima $(\uparrow)$ e spin para baixo $(\downarrow)$ e os $\varphi^{\prime} s$ são funções somente das coordenadas espaciais de um elétron.

É interessante observar que o uso do determinate de Slater para obter $\Psi_{0}$ garante automaticamente sua antissimetria, o que está de acordo com o princípio de exclusão de Pauli. Com a utilização da função de onda obtida através do determinante de Slater 
(proposta feita por Fock) o método passou a ser chamado método de Hartree-Fock $(\mathrm{HF})$.

O método de HF, além de considerar que a função de onda que descreve um sistema molecular é um determinate de Slater, ele envolve a utilização do princípio variacional [39,44] para a obtenção dos spin-orbitais moleculares, $\chi^{\prime} s$, que conduzem a um minímo o valor médio do operador hamiltoniano. Desta forma, devemos minimizar o funcional $E(\chi)$, que é o valor esperado do operador hamiltoniano (2.4), calculado no estado $\Psi_{0}$. Pode-se mostrar 44,47 que a dependência deste funcional com os spin-orbitais é dada da seguinte forma:

$$
E(\chi)=\sum_{i}\left\langle\chi_{i}|h| \chi_{i}\right\rangle+\frac{1}{2} \sum_{i, j}\left(\left\langle\chi_{i} \chi_{j} \mid \chi_{i} \chi_{j}\right\rangle-\left\langle\chi_{i} \chi_{j} \mid \chi_{j} \chi_{i}\right\rangle\right)
$$

onde h é o operador de um elétron,

$$
h\left(\mathbf{x}_{i}\right)=-\frac{1}{2} \nabla_{i}^{2}-\sum_{A=1}^{M} \frac{Z_{A}}{r_{i A}}
$$

O segundo membro na equação 2.22 engloba integrais de dois elétrons, em que o primeiro termo é a repulsão coulombiana entre dois elétrons nos orbitais $i$ e $j$, e o segundo termo é a interação de troca entre os elétrons, nesses orbitais.

$$
\left\langle\chi_{i} \chi_{j} \mid \chi_{k} \chi_{l}\right\rangle=\int \chi_{i}^{*}\left(\mathbf{x}_{1}\right) \chi_{j}^{*}\left(\mathbf{x}_{2}\right) \frac{1}{r_{12}} \chi_{k}\left(\mathbf{x}_{1}\right) \chi_{l}\left(\mathbf{x}_{2}\right) d \mathbf{x}_{1} d \mathbf{x}_{2}
$$

A minimização da equação 2.22 é feita sob a restrição de que os spin-orbitais moleculares permaneçam ortonormais, ou seja,

$$
\left\langle\chi_{i} \mid \chi_{j}\right\rangle-\delta_{i j}=0
$$

Problemas deste tipo, onde condições de vínculo se fazem presentes, podem ser resolvidos utilizando-se a técnica dos multiplicadores indeterminados de Lagrange 48. 
O método consiste em minimizar o funcional

$$
L(\chi)=E(\chi)-\sum_{i, j} \epsilon_{i j}\left(\left\langle\chi_{i} \mid \chi_{j}\right\rangle-\delta_{i j}\right)
$$

onde os coeficientes $\epsilon_{i j}$ são os multiplicadores de Lagrange. Minimizando o funcional, obtemos:

$$
f\left(\mathbf{x}_{1}\right) \chi_{i}\left(\mathbf{x}_{1}\right)=\sum_{j=1}^{N} \epsilon_{i j} \chi_{j}\left(\mathbf{x}_{1}\right)
$$

sendo $f$ o operador de Fock, que é dado por:

$$
f\left(\mathbf{x}_{1}\right)=h\left(\mathbf{x}_{1}\right)+\sum_{j=1}^{N}\left[\mathcal{J}_{j}\left(\mathbf{x}_{1}\right)-\mathcal{K}_{j}\left(\mathbf{x}_{1}\right)\right]
$$

onde h é um operador de uma partícula e no somatório temos os operadores de Coulomb, $\mathcal{J}_{j}$, e de troca, $\mathcal{K}_{j}$. Os operadores $\mathcal{J}_{j}$ e $\mathcal{K}_{j}$ são operadores de duas partículas que produzem integrais do tipo da equação 2.24 e podem ser escritos como:

$$
\begin{aligned}
& \mathcal{J}_{j}\left(\mathbf{x}_{1}\right) \chi_{i}\left(\mathbf{x}_{1}\right)=\left[\int \chi_{j}^{*}\left(\mathbf{x}_{2}\right) \frac{1}{r_{12}} \chi_{j}\left(\mathbf{x}_{2}\right) d \mathbf{x}_{2}\right] \chi_{i}\left(\mathbf{x}_{1}\right) \\
& \mathcal{K}_{j}\left(\mathbf{x}_{1}\right) \chi_{i}\left(\mathbf{x}_{1}\right)=\left[\int \chi_{j}^{*}\left(\mathbf{x}_{2}\right) \frac{1}{r_{12}} \chi_{i}\left(\mathbf{x}_{2}\right) d \mathbf{x}_{2}\right] \chi_{i}\left(\mathbf{x}_{1}\right)
\end{aligned}
$$

Na equação (2.29) o operador de Coulomb descreve a repulsão clássica coulombiana entre duas nuvens eletrônicas. O operador de troca 2.30 faz a mudança entre os elétrons do orbital $j$ e do orbital em que o operador atua e está relacionado com a correlação de troca, ou seja, à correlação no movimento dos elétrons com spins paralelos 44 .

Diagonalizando a matriz $\epsilon$, equação (2.27), através de uma transformação unitária, obteremos um novo conjunto de spin-orbitais, que serão os autovetores do operador de 
Fock, e os $\epsilon_{i}^{\prime} s$ podem ser interpretados como sendo as energias associadas a esses orbitais, onde o operador $f$ representa a energia sentida por um elétron. Após fazermos isso, a equação (2.27) é reescrita da seguinte forma:

$$
f\left(\mathbf{x}_{1}\right) \chi_{i}\left(\mathbf{x}_{1}\right)=\epsilon_{i} \chi_{i}\left(\mathbf{x}_{1}\right)
$$

Conhecida como equação canônica de Hartree-Fock.

Essa é uma equação de autovalores onde $f$, conhecido como operador de Fock, é um operador hermitiano; todos seus autovalores são reais e as autofunções pertencentes a diferentes autovalores são mutuamente ortogonais.

O operador de Fock, $f$, pode ser escrito como:

$$
f\left(\mathbf{x}_{i}\right)=-\frac{1}{2} \nabla_{i}^{2}-\sum_{A=1}^{M} \frac{Z_{A}}{r_{i A}}+v^{H F}\left(\mathbf{x}_{i}\right)
$$

O termo final na equação 2.32 ), $v^{H F}$ (definido por $2 \mathcal{J}_{j}-\mathcal{K}_{j}$ ), é o potencial de Hartree-Fock que representa o potencial médio do $i$-ésimo elétron na presença de todos os outros elétrons. Assim, o método de HF é uma aproximação de campo médio. Por esse motivo, nessa aproximação, perde-se a correlação eletrônica.

A importância do método de HF para a química teórica se dá não somente pelos seus resultados, mas por ser o fundamento para as aproximações posteriores que incluem os efeitos de correlação eletrônica, chamadas de pós-HF.

O procedimento para resolver a equação de HF (2.31) é através de um método iterativo chamado método do campo autoconsistente (Self-Consistent Field - SCF) [50]. O método consiste em escolher spin-orbitais iniciais por tentativa e a partir daí construir o potencial efetico $v^{H F}$ e resolver as equações, possibilitando a obtenção de um novo conjunto de spin-orbitais. A sequencia continua até que se obtenha um conjunto de funções, que comparado ao anterior, não apresente nenhuma mudança. 
Neste ponto considera-se que o cálculo convergiu.

\subsubsection{Equação de Hartree-Fock-Roothaan}

Para átomos, o problema de resolver a equação de Hartree-Fock é simplificado devido à simetria esférica [51]. No caso de moléculas a situação é mais complexa, já que essa simetria é perdida. Portanto, esse procedimento se mostrou computacionalmente inadequado para sistemas moleculares, pela dificuldade de representação dos orbitais moleculares. Esse problema foi resolvido em 1951 quando Roothaan apresentou uma abordagem matricial para a resolução da equação de HF |50|. Nessa formulação matricial do método HF, a essência é expandir os spin-orbitais moleculares em um conjunto de funções base conhecidas. A grande maioria dos cálculos de estrutura eletrônica é baseada na teoria em que aproximamos os orbitais moleculares por uma combinação linear de orbitais atômicos(Linear Combination of Atomic Orbitals, LCAO ) dados a priori.

Eliminando as coordenadas de spin do problema, nós retornamos aos orbitais espaciais, $\phi^{\prime} s$. Considerando moléculas de camada fechada, onde todos os orbitais moleculares são duplamente ocupados, a equação de HF em termos dos orbitais espaciais pode ser escrita como:

$$
f\left(\mathbf{r}_{1}\right) \phi_{i}\left(\mathbf{r}_{1}\right)=\epsilon_{i} \phi_{i}\left(\mathbf{r}_{1}\right)
$$

com o operador de Fock dado por:

$$
f\left(\mathbf{r}_{1}\right)=h\left(\mathbf{r}_{1}\right)+\sum_{j=1}^{N / 2}\left[2 \mathcal{J}_{j}\left(\mathbf{r}_{1}\right)-\mathcal{K}_{j}\left(\mathbf{r}_{1}\right)\right]
$$

onde o fator 2 surge porque, para os mesmos orbitais, coexistem dois elétrons.

Usando um conjunto de orbitais $\mathrm{g}_{\nu}(\mathbf{r})$ podemos expandir os orbitais moleculares, $\phi^{\prime} s$, da seguinte forma: 


$$
\phi_{i}(\mathbf{r})=\sum_{\nu=1}^{k} C_{\nu i} g_{\nu}(\mathbf{r})
$$

onde $k$ é o número de funções do conjunto e os $\mathrm{C}_{\nu i}$ 's são os coeficientes a serem determinados.

As funções mais utilizadas em cálculos moleculares são as chamadas funções tipo gaussianas, principalmente por causa da facilidade de se calcular integrais utilizando este tipo de função.

Substituindo a expansão 2.35 na equação de Hartree-Fock 2.33), temos:

$$
f\left(\mathbf{r}_{1}\right) \sum_{\nu=1}^{k} C_{\nu i} g_{\nu}\left(\mathbf{r}_{1}\right)=\epsilon_{i} \sum_{\nu=1}^{k} C_{\nu i} g_{\nu}\left(\mathbf{r}_{1}\right)
$$

multiplicando essa equação por $g_{\mu}^{*}\left(\mathbf{r}_{1}\right)$ e integrando, temos:

$$
\sum_{\nu=1}^{k} C_{\nu i}\left\langle g_{\mu}\left(\mathbf{r}_{1}\right)\left|f\left(\mathbf{r}_{1}\right)\right| g_{\nu}\left(\mathbf{r}_{1}\right)\right\rangle=\epsilon_{i} \sum_{\nu=1}^{k} C_{\nu i}\left\langle g_{\mu}\left(\mathbf{r}_{1}\right) \mid g_{\nu}\left(\mathbf{r}_{1}\right)\right\rangle
$$

Definindo os elementos da matriz de superposição,

$$
S_{\mu \nu}=\left\langle g_{\mu}\left(\mathbf{r}_{1}\right) \mid g_{\nu}\left(\mathbf{r}_{1}\right)\right\rangle
$$

e da matriz de Fock,

$$
F_{\mu \nu}=\left\langle g_{\mu}\left(\mathbf{r}_{1}\right)\left|f\left(\mathbf{r}_{1}\right)\right| g_{\nu}\left(\mathbf{r}_{1}\right)\right\rangle
$$

podemos então escrever,

$$
\sum_{\nu=1}^{k} F_{\mu \nu} C_{\nu i}=\epsilon_{i} \sum_{\nu=1}^{k} S_{\mu \nu} C_{\nu i}
$$

Em forma matricial, temos: 


$$
\mathbf{F C}=\mathbf{S C} \epsilon
$$

onde $\epsilon$ é uma matriz diagonal que contém as energias orbitais. Esta equação matricial é comumente chamada equação de Hartree-Fock-Roothaan. O que devemos fazer agora é ortogonalizar a matriz $\mathbf{S}$ de modo que a equação de Hartree-Fock-Roothaan tenha a forma de uma equação de autovalores matricial canônica que possa ser resolvida com relativa facilidade. Como $\mathbf{S}$ é hermitiana (igual a sua transposta conjugada), ela pode ser diagonalizada por uma transformação unitária. Essa matriz unitária é usada para obter uma nova matriz de Fock e uma nova matriz de coeficientes e obtemos assim a equação de autovalores. Essa nova equação é resolvida ortogonalizando-se a matriz de Fock resultante.

O método de HF trata o problema de interação elétron-elétron de uma forma média, ou como uma interação autoconsistente. Cada elétron participa do potencial que dá origem ao movimento de todos os elétrons. Isto significa que não há um tratamento detalhado onde o movimento de cada elétron é correlacionado com o movimento de todos os outros. A inclusão da contribuição do movimento correlacionado é a principal preocupação dos métodos ab initio. Define-se a contribuição da energia de correlação como a diferença entre a energia exata, não relativística, e a energia de Hartree-Fock [52],

$$
E_{\text {corr }}=E_{\text {exata }}-E_{H F}
$$

É importante mencionar que uma solução regular da equação de Hartree-Fock pode obter até $99 \%$ da energia total exata não relativística. A correlação é obtida ou por uma superposição de configurações ou por tratamentos perturbativos, este último será discutido a seguir. 


\subsubsection{Funções de Base}

Como mostrado na equação 2.35 o orbital molecular pode ser construido através de uma combinação linear de funções de base, onde essas funções são orbitais atômicos. Dois tipos de funções de base são comumentes utilizados para o cálculo de estrutura eletrônica. São elas: funções de Slater (Slater Type Orbitals, STO's) e as funções Gaussianas (Gaussian Type Orbitals, GTO’s). Há também as funções que combinam as funções de Slater e as funções Gaussianas. Esse conjunto de bases, STO-KG consiste em expandir os orbitais do tipo Slater em K funções gaussianas primitivas,

$$
\chi_{m}^{S T O}=\sum_{n}^{K} d_{m n} \chi_{n}^{G T O}
$$

Aqui, a função de base formada pela combinação linear de funções gaussianas é definida como uma base contraída e cada gaussiana que compõem essa combinação linear é definida como primitiva.

Huzinaga [53] e Pople e colaboradores [54], constataram que a melhor combinação entre a precisão e a velocidade para as funções de bases obtidas pela combinação linear de gaussianas foi obtido com $\mathrm{K}=3$. Essa função base, $\mathrm{STO}-3 \mathrm{G}$, ficou conhecida como base mínima ou single - $\zeta$, em que há apenas uma função de base definida para cada tipo de orbital. Embora a escolha da base mínima seja satisfatória para alguns sistemas ela apresenta limitações, nesse sentido, surgem funções de base em que o orbital atômico será representado a partir de duas funções contraídas, esse novo conjunto de funções de base é denominado double $-\zeta$. O mesmo raciocínio é seguido para construir funções multiple - $\zeta$. Há também o conjunto de funções de base split-valence em que os orbitais internos são representados por funções single ou double - $\zeta$ e possuem um número maior de funções de base para os orbitais de valência.

Neste trabalho realizamos cálculos com funções de base que usam o esquema de contração segmentado, utilizado por Pople, em que cada função de base primitiva 
contribui somente para uma função contraída.

A designação utilizado por Pople e colaboradores [55] para representar as funções de base contraídas é:

- 6-31G: É uma função split-valence, em que a região próxima ao núcleo é representada pela contração de seis gaussianas, a região interna dos orbitais da camada de valência é representada pela contração de 3 gaussianas e a parte externa da camada de valência é representada pela contração de uma gaussiana;

- 6-31G*: (*) Indica que foi adicionada uma função tipo-d para polarizar a função tipo- $p$ na base 6-31G;

- 6-31G**: (**) Indica que foi adicionado funções de polarização tipo-d para os átomos pesados e tipo- $p$ para os hidrogênios;

- 6-31+G**: $(+)$ Indica que foi adicionado funções difusas nos átomos pesados;

- 6-31++ $\mathrm{G}^{* *}:(++)$ Indica que foi adicionado funções difusas nos átomos pesados e também nos átomos de hidrogênio.

Os cálculos em nível HF e pós-HF realizados neste trabalho foram feitos usando o programa Gaussian [56. Na próxima seção discutiremos métodos pós-HF que usam teoria de perturbações para incluir a energia de correlação negligenciada no método HF. Como o nome sugere, pós-HF, esses métodos têm como ponto de partida as aproximações propostas por HF, buscando melhorar os seus resultados.

\subsection{Aproximações Perturbativas}

\subsubsection{Teoria de Perturbação de Rayleigh-Schrödinger}

Dentre as formulações perturbativas básicas, a mais popular é a Teoria de Pertubação de Rayleigh-Schrödinger (TPRS) |47], cuja idéia central é assumir que o hamil- 
toniano do sistema de interesse pode ser dividido em duas partes:

$$
\mathcal{H}=\mathcal{H}_{0}+\mathcal{V}
$$

onde $\mathcal{H}_{0}$, hamiltoniano não perturbado, difere pouco de $\mathcal{H}$ e os seus autovalores e autovetores são conhecidos,

$$
\mathcal{H}_{(0)}\left|\Psi_{n}^{(0)}\right\rangle=E_{n}^{(0)}\left|\Psi_{n}^{(0)}\right\rangle \quad n=0,1,2,3, \ldots
$$

O problema consiste em resolver a equação de autovalor para o hamiltoniano $\mathcal{H}$,

$$
\mathcal{H}\left|\Psi_{n}\right\rangle=E_{n}\left|\Psi_{n}\right\rangle
$$

onde $n$ indica o estado a ser corrigido. Substituindo a equação (2.44) na (2.46), temos:

$$
\left(\mathcal{H}_{0}+\mathcal{V}\right)\left|\Psi_{n}\right\rangle=E_{n}\left|\Psi_{n}\right\rangle
$$

de maneira que os autovetores e autovalores de $\mathcal{H}$ podem ser obtidos partindo-se dos autovalores e autovetores conhecidos de $\mathcal{H}_{0}$ e dos elementos de matriz de $\mathcal{V}$ na base dos autovetores de $\mathcal{H}_{0}$.

Para que se tenha o ordenamento das correções na energia e na função de onda, é conveniente escrever a equação (2.44) na seguinte forma:

$$
\mathcal{H}=\mathcal{H}_{0}+\lambda \mathcal{V}
$$

onde $\lambda$ é um parâmetro de ordenamento, tal que para $\lambda \rightarrow 0$, o autovalor exato $E_{n}$ tende ao autovalor não perturbado $E_{n}^{(0)}$.

Como o hamiltoniano $\mathcal{H}$ depende de $\lambda$, fica claro que tanto a energia como a função de onda perturbadas dependerão igualmente de $\lambda$. 
Expandindo a função exata $\left|\Psi_{n}\right\rangle$ e a energia exata $E_{n}$ em uma série de Taylor em torno de $\lambda=0$, temos:

$$
E_{n}(\lambda)=E_{n}^{(0)}+\lambda\left(\frac{d E_{n}}{d \lambda}\right)_{\lambda=0}+\lambda^{2} \frac{1}{2 !}\left(\frac{d^{2} E_{n}}{d \lambda^{2}}\right)_{\lambda=0}+\ldots
$$

Definindo que:

$$
E_{n}^{(1)}=\left(\frac{d E_{n}}{d \lambda}\right)_{\lambda=0}, \quad E_{n}^{(2)}=\frac{1}{2 !}\left(\frac{d^{2} E_{n}}{d \lambda^{2}}\right)_{\lambda=0}, \ldots
$$

podemos reescrever a equação 2.49 ,

$$
E_{n}=E_{n}^{(0)}+\lambda E_{n}^{(1)}+\lambda^{2} E_{n}^{(2)}+\ldots
$$

onde $E_{n}^{(j)}$ é a correção de ordem $j$ na energia. Analogamente, podemos escrever a expansão para a função de onda $\Psi_{n}$,

$$
\Psi_{n}=\left|\Psi_{n}^{(0)}\right\rangle+\lambda\left|\Psi_{n}^{(1)}\right\rangle+\lambda^{2}\left|\Psi_{n}^{(2)}\right\rangle+\ldots
$$

onde $\left|\Psi_{n}^{(j)}\right\rangle$ é a correção de ordem $j$ na função de onda.

Aqui vamos supor que o estado não pertubado, $E_{n}^{(0)}$, seja não degenerado e que suas autofunções sejam ortonormais, ou seja,

$$
\left\langle\Psi_{i}^{(0)} \mid \Psi j^{(0)}\right\rangle=\delta_{i j}
$$

Além disso, devemos impor a normalização intermediária,

$$
\left\langle\Psi_{n}^{(0)} \mid \Psi_{n}\right\rangle=1
$$

Agora, as correções na energia e na função de onda são obtidas substituindo-se as equações 2.51) e 2.52 nas equações 2.47) e 2.48), temos: 


$$
\begin{array}{r}
\left(\mathcal{H}_{0}+\lambda \mathcal{V}\right)\left(\left|\Psi_{n}^{(0)}\right\rangle+\lambda\left|\Psi_{n}^{(1)}\right\rangle+\lambda^{2}\left|\Psi_{n}^{(2)}\right\rangle+\ldots\right)= \\
\left(E_{n}^{(0)}+\lambda E_{n}^{(1)}+\lambda^{2} E_{n}^{(2)}+\ldots\right)\left(\left|\Psi_{n}^{(0)}\right\rangle+\lambda\left|\Psi_{n}^{(1)}\right\rangle+\lambda^{2}\left|\Psi_{n}^{(2)}\right\rangle+\ldots\right)
\end{array}
$$

Igualando-se os termos de mesma potência em $\lambda$, obtemos:

$$
\begin{aligned}
\lambda^{0} \rightarrow \mathcal{H}_{0}\left|\Psi_{n}^{(0)}\right\rangle=E_{n}^{(0)}\left|\Psi_{n}^{(0)}\right\rangle & \\
\lambda^{1} \rightarrow\left(\mathcal{H}_{0}-E_{n}^{(0)}\right)\left|\Psi_{n}^{(1)}\right\rangle & =\left(E_{n}^{(1)}-\mathcal{V}\right)\left|\Psi_{n}^{(0)}\right\rangle \\
\lambda^{2} \rightarrow\left(\mathcal{H}_{0}-E_{n}^{(0)}\right)\left|\Psi_{n}^{(2)}\right\rangle & =\left(E_{n}^{(1)}-\mathcal{V}\right)\left|\Psi_{n}^{(1)}\right\rangle+E_{n}^{2}\left|\Psi_{n}^{(0)}\right\rangle \\
\lambda^{3} \rightarrow\left(\mathcal{H}_{0}-E_{n}^{(0)}\right)\left|\Psi_{n}^{(3)}\right\rangle & \left.=\left(E_{n}^{(1)}-\mathcal{V}\right)\left|\Psi_{n}^{(2)}\right\rangle+E_{n}^{2}\left|\Psi_{n}^{(1)}+E_{n}^{3}\right| \Psi_{n}^{(0)}\right\rangle \\
& \vdots \\
\lambda^{n} \rightarrow\left(\mathcal{H}_{0}-E_{n}^{(0)}\right)\left|\Psi_{n}^{(n)}\right\rangle & \left.=\left(E_{n}^{(1)}-\mathcal{V}\right)\left|\Psi_{n}^{(n-1)}\right\rangle+E_{n}^{2}\left|\Psi_{n}^{(n-2)}+\ldots+E_{n}^{n}\right| \Psi_{n}^{(0)}\right\rangle
\end{aligned}
$$

Multiplicando as equações acima por $\left\langle\Psi_{n}^{(0)}\right|$ e utilizando o resultado

$$
\left\langle\Psi_{n}^{(0)}\right|\left(\mathcal{H}_{0}-E_{n}^{(0)}\right)=0
$$

obtemos:

$$
\begin{aligned}
E_{n}^{(1)} & =\left\langle\Psi_{n}^{(0)}|\mathcal{V}| \Psi_{n}^{(0)}\right\rangle \\
E_{n}^{(2)} & =\left\langle\Psi_{n}^{(0)}\left|\mathcal{V}-E_{0}^{(1)}\right| \Psi_{n}^{(1)}\right\rangle \\
E_{n}^{(3)} & =\left\langle\Psi_{n}^{(0)}\left|\mathcal{V}-E_{0}^{(1)}\right| \Psi_{n}^{(2)}\right\rangle-E_{n}^{(2)}\left\langle\Psi_{n}^{(0)} \mid \Psi_{n}^{(1)}\right\rangle \\
& \vdots \\
E_{n}^{(n)} & =\left\langle\Psi_{n}^{(0)}\left|\mathcal{V}-E_{0}^{(1)}\right| \Psi_{n}^{(n-1)}\right\rangle-E_{n}^{(2)}\left\langle\Psi_{n}^{(0)} \mid \Psi_{n}^{(n-2)}\right\rangle-\ldots-E_{n}^{(n-1)}\left\langle\Psi_{n}^{(0)} \mid \Psi_{n}^{(1)}\right\rangle
\end{aligned}
$$

Assim, os conjuntos de equações 2.56 e 2.58 permitem, em princípio, obter as correções em qualquer ordem na função de onda e na energia. As expressões contidas 
na equação (2.58) podem ser simplificadas utilizando-se a normalização intermediária 2.54 ,

$$
\begin{aligned}
E_{n}^{(1)} & =\left\langle\Psi_{n}^{(0)}|\mathcal{V}| \Psi_{n}^{(0)}\right\rangle \\
E_{n}^{(2)} & =\left\langle\Psi_{n}^{(0)}|\mathcal{V}| \Psi_{n}^{(1)}\right\rangle \\
E_{n}^{(3)} & =\left\langle\Psi_{n}^{(0)}|\mathcal{V}| \Psi_{n}^{(2)}\right\rangle \\
& \vdots \\
E_{n}^{(n)} & =\left\langle\Psi_{n}^{(0)}|\mathcal{V}| \Psi_{n}^{(n-1)}\right\rangle
\end{aligned}
$$

As equações (2.59) nos mostram que para se obter a energia corrigida até a ordem $j$ é necessário o conhecimento da função de onda até ordem $j-1$. Na verdade, é possível mostrar que o conhecimento da correção de ordem $j$, na função de onda, nos permite determinar a correção na energia até a ordem $2 j+1[52]$.

Como nem sempre é possível obter a solução exata $\left|\Psi_{n}^{(j)}\right\rangle$ para o conjunto de equações diferenciais não homogêneas dadas na equação (2.57), o que se faz é uma expansão das funções de onda perturbadas $\left|\Psi_{n}^{(j)}\right\rangle$, para isso usa-se como base nessa expansão o conjunto completo gerado pelas autofunções do hamiltoniano não perturbado, $\left|\Psi_{i}^{(0)}\right\rangle$,

$$
\left|\Psi_{n}^{(j)}\right\rangle=\sum_{i} c_{i}^{j}\left|\Psi_{i}^{(0)}\right\rangle
$$

Para ilustrar as correções na função de onda e na energia, vamos obter a correção de primeira ordem, $j=1$, para a função de onda e a correção de segunda ordem para a energia. Multiplicando por $\left|\Psi_{k}^{(0)}\right\rangle$ a expressão para potência $1, \lambda^{1}$, na equação (2.56), obtemos:

$$
\left\langle\Psi_{k}^{(0)}\left|\left(\mathcal{H}_{0}-E_{n}^{(0)}\right)\right| \Psi_{n}^{(1)}\right\rangle=\left\langle\Psi_{k}^{(0)}\left|\left(E_{n}^{(1)}-\mathcal{V}\right)\right| \Psi_{n}^{(0)}\right\rangle
$$


usando o fato de que $\mathcal{H}_{0}$ é hermitiano e substituindo a equação 2.60 na 2.61), fazendo $j=1$, temos:

$$
\left(E_{k}^{(0)}-E_{n}^{(0)}\right)\left\langle\Psi_{k}^{(0)}\left|\sum_{i} c_{i}^{(1)}\right| \Psi_{i}^{(0)}\right\rangle=E_{n}^{(1)}\left\langle\Psi_{k}^{(0)} \mid \Psi_{n}^{(0)}\right\rangle-\left\langle\Psi_{k}^{(0)}|\mathcal{V}| \Psi_{n}^{(0)}\right\rangle
$$

Usando o fato de que os autovetores da hamiltoniana não perturbada são ortonormais, equação (2.53), obtemos:

$$
\left(E_{k}^{(0)}-E_{n}^{(0)}\right) \sum_{i} c_{i}^{(1)} \delta_{k i}=-\left\langle\Psi_{k}^{(0)}|\mathcal{V}| \Psi_{n}^{(0)}\right\rangle
$$

$\log \mathrm{O}$

$$
c_{k}^{(1)}=\frac{\left\langle\Psi_{k}^{(0)}|\mathcal{V}| \Psi_{n}^{(0)}\right\rangle}{E_{n}^{(0)}-E_{k}^{(0)}}
$$

substituindo a equação 2.64 na equação 2.60), fazendo $j=1$, obtemos a correção em primeira ordem para a função de onda:

$$
\left|\Psi_{n}^{(1)}\right\rangle=\sum_{k} \frac{\left\langle\Psi_{k}^{(0)}|\mathcal{V}| \Psi_{n}^{(0)}\right\rangle}{E_{n}^{(0)}-E_{k}^{(0)}}\left|\Psi_{k}^{(0)}\right\rangle
$$

A correção em segunda ordem na energia é obtida substituindo a equação 2.65 na expressão para $E_{n}^{(2)}$ na equação 2.59 ,

$$
E_{n}^{(2)}=\sum_{k \neq n} \frac{\left|\left\langle\Psi_{k}^{(0)}|\mathcal{V}| \Psi_{n}^{(0)}\right\rangle\right|^{2}}{E_{n}^{(0)}-E_{k}^{(0)}}
$$

\subsubsection{Teoria de Perturbação Møller-Plesset}

A forma usual de perturbação em química quântica usa TPRS com a partição do hamiltoniano eletrônico proposta por Møller-Plesset [57], e é comumente chamada teoria de perturbação Møller-Plesset (MP) [58. 
A teoria MP considera como o hamiltoniano não perturbado, $\mathcal{H}_{0}$, como a soma de operadores de Fock tal como definido na equação 2.28,

$$
\mathcal{H}_{0}=\sum_{i=1}^{N} f(i)
$$

A perturbação, $\mathcal{V}$, é então obtida pela diferença entre o hamiltoniano molecular dado pela equação (2.4), $\mathcal{H}$, e o hamiltoniano não perturbado, $\mathcal{H}_{0}$,

$$
\mathcal{V}=\mathcal{H}-\mathcal{H}_{0}=\sum_{i<j} \frac{1}{r_{i j}}-\sum_{i} v^{H F}(i)
$$

onde

$$
v^{H F}(i)=\sum_{j}\langle j \| j\rangle=\sum_{j}\left[\mathcal{J}_{j}(i)-\mathcal{K}_{j}(i)\right]
$$

Com $\mathcal{H}_{0}$ definido pela equação (2.67), temos que a função de onda Hartree-Fock (2.19), $\Psi_{0}$, é autovetor de $\mathcal{H}_{0}$ com autovalor $E_{0}^{(0)}$, dado pela soma das energias orbitais de todos os spin-orbitais,

$$
\begin{aligned}
\mathcal{H}_{0} \Psi_{0} & =\sum_{i=1}^{N} f(i)\left|\chi_{1} \chi_{2} \ldots \chi_{N}\right\rangle \\
& =\left(\epsilon_{1}+\epsilon_{2}+\ldots+\epsilon_{N}\right)\left|\chi_{1} \chi_{2} \ldots \chi_{N}\right\rangle \\
& =E_{0}^{(0)} \Psi_{0}
\end{aligned}
$$

com

$$
E_{0}^{(0)}=\sum_{c=1}^{N} \epsilon_{c}
$$

Usando as expressões gerais para TPRS podemos calcular as várias correções para um sistema de $\mathrm{N}$ elétrons. A correção em primeira ordem para a energia do estado fundamental, $E_{0}^{(1)}$, é dada por: 


$$
E_{0}^{(1)}=\left\langle\Psi_{0}|\mathcal{V}| \Psi_{0}\right\rangle=\left\langle\Psi_{0}\left|\sum_{i<j} \frac{1}{r_{i j}}\right| \Psi_{0}\right\rangle-\left\langle\Psi_{0}\left|\sum_{i, j=1}\left[\mathcal{J}_{j}(i)-\mathcal{K}_{j}(i)\right]\right| \Psi_{0}\right\rangle
$$

Usando as regras de Condo-Slater [58,59|, temos:

$$
\begin{gathered}
\left\langle\Psi_{0}\left|\sum_{i<j} \frac{1}{r_{i j}}\right| \Psi_{0}\right\rangle=\frac{1}{2} \sum_{i, j=1}^{N}\left\langle\chi_{i} \chi_{j} \| \chi_{i} \chi_{j}\right\rangle \\
\left\langle\Psi_{0}\left|\sum_{i, j=1}\left[\mathcal{J}_{j}(i)-\mathcal{K}_{j}(i)\right]\right| \Psi_{0}\right\rangle=\sum_{i, j=1}^{N}\left\langle\chi_{i} \chi_{j} \| \chi_{i} \chi_{j}\right\rangle
\end{gathered}
$$

com

$$
\left\langle\chi_{i} \chi_{j} \| \chi_{i} \chi_{j}\right\rangle=\left\langle\chi_{i} \chi_{j} \mid \chi_{i} \chi_{j}\right\rangle-\left\langle\chi_{i} \chi_{j} \mid \chi_{j} \chi_{i}\right\rangle
$$

e a correção de primeira ordem é:

$$
E_{0}^{(1)}=-\frac{1}{2} \sum_{i, j=1}^{N}\left\langle\chi_{i} \chi_{j} \| \chi_{i} \chi_{j}\right\rangle
$$

Logo, a energia para o estado fundamental de um sistema com N elétrons corrigida até primeira ordem é dada por:

$$
E_{0}=E_{0}^{(0)}+E_{0}^{(1)}=\sum_{i=1}^{N} \epsilon_{i}-\frac{1}{2} \sum_{i, j=1}^{N}\left\langle\chi_{i} \chi_{j} \| \chi_{i} \chi_{j}\right\rangle=E_{H F}
$$

que é a energia Hartree-Fock para o estado fundamental. Portanto, a energia de Hartree-Fock é correta até primeira ordem na MP. A correlação só se apresenta a partir da segunda ordem:

$$
E_{\text {corr }}=E_{0}^{(2)}+E_{0}^{(3)}+\ldots
$$


A equação (2.66), que dá a correção em segunda ordem na energia, no caso da partição de Møller-Plesset, exige o cálculo de elementos de matriz envolvendo o estado de referência de Hartree-Fock $\left|\Psi_{0}\right\rangle$ e as excitações $\left\{\left|\Psi_{a}^{r}\right\rangle,\left|\Psi_{a b}^{r s}\right\rangle, \ldots,\left|\Psi_{a b \ldots .}^{r s \ldots}\right\rangle\right\}$. Aqui, $\left|\Psi_{a}^{r}\right\rangle$ significa que são realizadas apenas excitações simples (a excitação eletrônica ocorre do orbital ocupado $a$ para o orbital virtual $r$ ), já o autovetor $\left|\Psi_{a b}^{r s}\right\rangle$ leva em conta excitações duplas e assim sucessivamente. Considerando apenas excitações simples, temos:

$$
\left\langle\Psi_{0}|\mathcal{V}| \Psi_{a}^{r}\right\rangle=\left\langle\Psi_{0}|\mathcal{H}| \Psi_{a}^{r}\right\rangle-\left\langle\Psi_{0}\left|\mathcal{H}_{0}\right| \Psi_{a}^{r}\right\rangle
$$

onde a primeira integral é nula pelo teorema de Brillouin [39] e a segunda parcela dá,

$$
\left\langle\Psi_{0}\left|\mathcal{H}_{0}\right| \Psi_{a}^{r}\right\rangle=\sum_{j} \epsilon_{j}\left\langle\Psi_{0} \mid \Psi_{a}^{r}\right\rangle=0, \quad\left\langle\Psi_{0} \mid \Psi_{a}^{r}\right\rangle=0
$$

o que resulta em

$$
\left\langle\Psi_{0}|\mathcal{V}| \Psi_{a}^{r}\right\rangle=0
$$

Assim, as excitações simples não contribuem para a correção de segunda ordem.

Para excitações triplas e superiores obtém-se que:

$$
\left\langle\Psi_{0}|\mathcal{V}| \Psi_{a b c}^{r s t}\right\rangle=\left\langle\Psi_{0}|\mathcal{V}| \Psi_{a b \ldots}^{r s \ldots}\right\rangle=0
$$

pois $\mathcal{V}$ contém apenas operadores de uma partícula $\left(V^{H F}(i)\right)$ e duas partículas $\left(1 / r_{i j}\right)$. Logo, essas excitações não contribuirão para a correção de segunda ordem.

Pelo que foi analisado anteriormente, observa-se que a correção de segunda ordem da energia do estado fundamental usando MP requer somente a inclusão de excitações duplas,

$$
E_{0}^{(2)}=\sum_{a<b} \sum_{r<s} \frac{\left|\left\langle\Psi_{0}|\mathcal{V}| \Psi_{a b}^{r s}\right\rangle\right|^{2}}{E_{0}^{(0)}-E_{a b}^{r s}}
$$

onde o valor do numerador é obtido usando a regra de Condo-Slater 


$$
\left\langle\Psi_{0}|\mathcal{V}| \Psi_{a b}^{r s}\right\rangle=\langle a b \| r s\rangle
$$

Logo,

$$
E_{0}^{(2)}=\sum_{a<b} \sum_{r<s} \frac{|\langle a b \| r s\rangle|^{2}}{\epsilon_{a}+\epsilon_{b}-\epsilon_{r}-\epsilon_{s}}
$$

A equação (2.85) pode ser escrita como uma soma livre notando que a soma é simétrica em $a$ e $b$ e em $r$ e $s$, e que se anula se $a=b$ ou $r=s$. Assim,

$$
E_{0}^{(2)}=\frac{1}{4} \sum_{a<b} \sum_{r<s} \frac{|\langle a b \| r s\rangle|^{2}}{\epsilon_{a}+\epsilon_{b}-\epsilon_{r}-\epsilon_{s}}
$$

conhecida como forma de Brandow $|60|$.

Neste trabalho realizamos cálculos usando a teoria de perturbação MP até segunda ordem, que é denotada por MP2. Vale lembrar que por não ser fundamentada no princípio variacional, as energias obtidas utilizando MP podem ser inferiores à energia exata.

\subsection{Teoria do Funcional da Densidade}

Uma outra forma de resolver a equação de Schrödinger para um sistema multieletrônico é usando a sua densidade eletrônica. A aplicação da Teoria do Funcional da Densidade (DFT) em átomos, sólidos e moléculas tem crescido bastante e, nas últimas décadas, tem se mostrado um importante método para o cálculo de propriedades eletrônicas e estruturais para o estado fundamental. A grande vantagem dessa metodologia, quando comparada com os métodos ab inito padrões, que são baseados no método Hartree-Fock, é apresentar um custo computacional relativamente baixo.

O uso da densidade eletrônica $\rho(\mathbf{r})$ como variável básica foi legitimada com a publicação dos teoremas de Hohemberg e Kohn (HK) em 1964 sobre gás de elétrons não 
homogêneos [61], tornando explícita a repulsão elétron-elétron de Coulomb e definindo uma nova função universal $F[\rho]$.

Na DFT, os elétrons interagem entre si e com um potencial externo, $v(\mathbf{r})$. O primeiro teorema de HK estabelece que esse potencial externo é um funcional único, a menos de uma constante, da densidade eletrônica $\rho(\mathbf{r})$. Isso implica que a densidade eletrônica de um sistema determina o potencial externo e o número de elétrons, N, e consequentemente, o hamiltoniano do sistema. Onde esse hamiltoniano para um sistema multieletrônico é escrito como:

$$
\mathcal{H}=\mathcal{T}+\mathcal{U}+\mathcal{V}
$$

onde $\mathcal{T}$ é o operador energia cinética; $\mathcal{U}$, é o operador de repulsão elétron-elétron, que inclui a repulsão Coulombiana e todos os termos não clássicos (troca e correlação); e $\mathcal{V}=\sum_{i}^{N} v\left(r_{i}\right)$, é o potencial externo sentido pelos elétrons, que para moléculas é devido as cargas dos núcleos.

A energia total do sistema é então obtida pelo valor esperado do hamiltoniano, $\langle\Psi|\mathcal{H}| \Psi\rangle$. E essa energia é um funcional da densidade eletrônica $\rho(\mathbf{r})$,

$$
\begin{aligned}
E[\rho] & =\langle\Psi|\mathcal{T}+\mathcal{U}+\mathcal{V}| \Psi\rangle \\
& =\langle\Psi|\mathcal{T}+\mathcal{U}| \Psi\rangle+\langle\Psi|\mathcal{V}| \Psi\rangle \\
& =F[\rho]+\int v(\mathbf{r}) \rho(\mathbf{r}) d^{3} r
\end{aligned}
$$

onde $F[\rho]$ é um funcional universal, ou seja, sua dependência com a densidade é a mesma para todos sistemas que possuam a mesma interação elétron-elétron.

O segundo teorema de HK obedece ao princípio variacional e estabelece que, havendo qualquer aproximação da densidade eletrônica, $\tilde{\rho}(\mathbf{r})$, tal que $\tilde{\rho}(\mathbf{r}) \geq 0$ e $\int \tilde{\rho}(\mathbf{r}) d^{3} r=N$, a energia total será sempre maior ou igual a energia exata do sistema, ou seja, 


$$
E[\tilde{\rho}]=F[\tilde{\rho}]+\int v(\mathbf{r}) \tilde{\rho}(\mathbf{r}) d^{3} r \geq E[\rho]=E_{0}
$$

onde $\tilde{\rho}(\mathbf{r})$ define seu próprio $\tilde{v}(\mathbf{r})$ e, consequentemente, o hamiltoniano $\tilde{H}$ e $\tilde{\Psi}\left(r_{1}, r_{2}, \ldots, r_{N}\right)$. Em termos, o segundo teorema diz que a energia do estado fundamental do sistema pode ser obtida minimizando a energia total com relação a densidade.

Os teoremas de HK nos garantem que a energia do estado fundamental, para um sistema multieletrônico, pode ser obtida através da sua densidade mas não nos ensina como isso deve ser feito na prática. Um procedimento para resolver essa questão foi proposto por Kohn e Sham em 1965 [62]. A idéia nesse procedimento é construir um sistema auxiliar de partículas independentes, como referência, que possua a mesma densidade do sistema interagente. Na seção seguinte fazemos uma discussão sobre a aproximação de Kohn-Sham (KS).

\subsubsection{Equações de Kohn-Sham}

A determinação da energia do estado fundamental de um sistema multieletrônico, sob um potencial externo, é feita usando a aproximação de Konh-Sham, que utiliza um sistema de partículas independentes como referência.

Kohn e Sham reescreveram a equação 2.90 tornando explícita a repulsão elétronelétron de Coulomb e definindo um novo funcional universal $G[\rho]$ :

$$
E[\rho]=G[\rho]+\frac{1}{2} \iint \frac{\rho(\mathbf{r}) \rho\left(\mathbf{r}^{\prime}\right)}{\left|\mathbf{r}-\mathbf{r}^{\prime}\right|} d^{3} r d^{3} r^{\prime}+\int \rho(\mathbf{r}) v(\mathbf{r}) d^{3} r
$$

onde

$$
G[\rho]=T_{s}[\rho]+E_{x c}[\rho]
$$

sendo $T_{s}[\rho]$ o funcional energia cinética de um sistema de elétrons que não interagem tendo a mesma densidade eletrônica do sistema de elétrons que interagem. Dessa forma, $E_{x c}[\rho]$ inclui não só o termo de interação elétron-elétron não-clássico (troca e 
correlação) mas também a parte residual da energia cinética, $T[\rho]-T_{s}[\rho]$, em que $T[\rho]$ é o funcional energia cinética exata para o sistema de elétrons que interagem. É interessante ressaltar que a fórmula funcional exata para $E_{x c}[\rho]$ não é simples, e nem mesmo conhecida. É nesse funcional que se concentram as aproximações da DFT prática.

Assim como no método Hartree-Fock, a idéia aqui é minimizar o funcional $E[\rho]$ sujeito ao vínculo de que a carga eletrônica total seja fixa, ou seja,

$$
\int \rho(\mathbf{r}) d^{3} r=N
$$

Fazendo a minimização, com a condição de extremo, incluindo o vínculo, cairemos em uma equação de autovalores dada por (ver referência [39|),

$$
\begin{aligned}
\left(-\frac{1}{2} \nabla^{2}+v^{K S}[\rho]\right) \Psi_{i}(\mathbf{r}) & =\epsilon_{i} \Psi_{i}(\mathbf{r}) \\
\text { ou } & \\
h^{K S} \Psi_{i}(\mathbf{r}) & =\epsilon_{i} \Psi_{i}(\mathbf{r})
\end{aligned}
$$

A equação (2.96) é conhecida como equação de Kohn-Sham (KS) e $h^{K S}$ é o hamiltoniano de KS (operador de uma partícula). O potencial efetivo de KS para uma partícula, $v^{K S}$, é dado por:

$$
v^{K S}=v(\mathbf{r})+\int \frac{\rho\left(\mathbf{r}^{\prime}\right)}{\left|\mathbf{r}-\mathbf{r}^{\prime}\right|} d^{3} r^{\prime}+v_{x c}(\rho)
$$

O primeiro termo na equação (2.97), $v(\mathbf{r})$, é o potencial externo (que para moléculas é devido às cargas dos núcleos). Em seguida vem o potencial de Hartree, que descreve a interação eletrostática entre os elétrons. Por último nós temos o potencial de troca-correlação $v_{x c}$, que inclui todos os efeitos não triviais de muitos corpos. Esse 
termo pode ser escrito como uma derivada funcional do funcional energia de trocacorrelação, $E_{x c}$ (ver referência [39]),

$$
v_{x c}[\rho]=\frac{\delta E_{x c}}{\delta \rho}
$$

Para obtermos $v^{K S}$, pela equação (2.97), é necessário conhecermos $v_{x c}$. Portanto, da equação (2.98) observa-se que é preciso fazermos uma escolha a priori do funcional troca-correlação, $E_{x c}[\rho]$. Se o funcional energia de troca-correlação, $E_{x c}[\rho]$, fosse conhecido exatamente, a resolução da equação de Kohn-Sham nos forneceria a densidade exata para um sistema de muitos corpos interagentes. Porém, $E_{x c}[\rho]$ é uma quantidade bastante complicada e deve ser aproximada para qualquer aplicação prática do esquema Konh-Shan.

A primeira aproximação para o termo $E_{x c}[\rho]$ foi proposta por Kohn e Shan em 1965 62. Essa aproximação é chamada de Aproximação Local da Densidade, LDA (do inglês, Local Density Approximation),

$$
E_{x c}^{L D A}[\rho]=\int \rho(\mathbf{r}) \epsilon_{x c}^{h}(\rho(\mathbf{r})) d^{3} r
$$

onde $\epsilon_{x c}^{h}(\rho)$ é a energia de troca-correlação, por elétron, de um gás de elétrons homogêneo de densidade $\rho=\rho(\mathbf{r})$. Nessa aproximação o funcional $E_{x c}[\rho]$ é local, ou seja, a densidade de energia num ponto $\mathbf{r}$ só dependerá da densidade eletrônica existente no mesmo ponto . Apesar de ser, por construção, uma aproximação local, a LDA produz bons resultados mesmo para sistemas não homogêneos, como átomos ou moléculas pequenas.

Como em sistemas reais a densidade não é homogênea, há um refinamento no método LDA, que é expressar o funcional $E_{x c}[\rho]$ em termos do gradiente da densidade de carga total. Essa aproximação é conhecida como expansão generalizada em termos de gradientes GGA (do inglês, Generalized Gradient Approximation), que tem a 
seguinte fórmula geral $63 \mid$ :

$$
E_{x c}^{G G A}[\rho]=\int f(\rho(\mathbf{r}), \nabla \rho(\mathbf{r})) d^{3} r
$$

Existem várias propostas para o funcional $E_{x c}^{G G A}$, as mais utilizadas, atualmente, são baseadas nos trabalhos de Perdew-Burke-Erzenhof [64], de Lee-Yang-Parr [65, 66], de Perdew e Wang [67, de Perdew [68] e de Becke [69].

Vários desses funcionais energia de troca-correlação foram desenvolvidos de forma a atender parte das restrições que o funcional exato deve possuir como, por exemplo, o comportamento assintótico para grandes distâncias. Esses funcionais apresentam, de modo geral, alguns parâmetros que são ajustados de maneira a reproduzir a energia de troca-correlação exata para alguns sistemas bem estudados, como um gás de elétrons uniforme, ou são ajustados para reproduzir a energia de troca-correlação obtida por outros métodos quânticos. No entanto, há funcionais que são puros como, por exemplo, PW91 (Perdew-Wang) |70| e PBE (Perdew-Burke-Erzenhof) 64.

Há também o funcional híbrido Hartree-Fock/DFT que foi proposto para incorporar a não localidade do método Hartree-Fock na DFT. A idéia dos métodos híbridos é a de incluir parte do termo de troca exato do método Hartree-Fock no funcional GGA.

Na linha dos funcionais híbridos, nós realizamos cálculos usando dois funcionais híbridos distintos: B3LYP (Becke's three-parameter exchange + Lee-Yang-Parr correlation) 66, 71 e BHandHLYP (Becke's half-and-half exchange + Lee-Yang-Parr correlation) [?]. A energia de troca-correlação, $E_{x c}$, para os dois funcionais difere apenas no termo de troca (exchange). O funcional B3LYP é composto por uma mistura contendo $20 \%$ de Hartree-Fock para a densidade de troca enquanto que o funcional BHandHLYP incorpora $50 \%$ de Hartree-Fock para a densidade de troca.

A energia de troca-correlação para o funcional B3LYP é escrita como: 


$$
E_{x c}=E_{x c}^{L D A}+a_{0}\left(E_{x}^{H F}-E_{x}^{L D A}\right)+a_{x}\left(E_{x}^{G G A}-E_{x}^{L D A}\right)+a_{c}\left(E_{c}^{G G A}-E_{c}^{L D A}\right)
$$

onde $a_{0}=0.20, a_{x}=0.72$, e $a_{c}=0.81$ são três parâmetros empíricos determinados pelo ajuste dos valores previstos para um conjunto de energias de atomização, potenciais de ionização, próton afinidade e energias atômicas. Os termos, $E_{x}^{G G A}$ e $E_{c}^{G G A}$ correspondem ao funcional de troca Becke88 [69] e ao funcional de correlação de LeeYang-Parr [65], respectivamente. O termo $E_{c}^{L D A}$ corresponde a aproximação proposta por Vosko-Wilk-Nusair (VWN) |72] para o funcional de correlação.

O funcional BHandHLYP é dado por:

$$
E_{x c}=0.5^{*} E_{x}^{H F}+0.5^{*} E_{x}^{B}+1.0^{*} E_{c}^{L Y P}
$$

onde $E_{x}^{H F}$ corresponde ao termo de troca Hartree-Fock, $E_{x}^{B}$ corresponde ao funcional de troca Becke88, e $E_{c}^{L Y P}$ corresponde ao funcional de correlação proposto por LeeYang-Parr.

\subsubsection{DFT Dependente do Tempo (TD-DFT)}

A DFT tem se mostrado bastante eficiente na descrição do estado fundamental de sistemas atômicos e moleculares. Devido ao sucesso da aplicação da DFT em estados estacionários, tem surgido o interesse em descrever estados dependentes do tempo (TD- Time Dependent) em termos do funcional da densidade. Problemas como processos de espalhamento atômico 73 e nuclear $\mid 74$ e fotoabsorção em átomos 75 têm sido tratados com sucesso nessa abordagem.

No trabalho de 1984, Runge e Gross [76] generalizaram o teorema de HohenbergKohn para o caso da equação de Schrödinger dependente do tempo,

$$
i \frac{\partial \Phi(\{\mathbf{r}\}, t)}{\partial t}=\mathcal{H}(\{\mathbf{r}\}, t) \Phi(\{\mathbf{r}\}, t), \quad \Phi\left(\{\mathbf{r}\} t_{0}\right)=\Phi_{0}
$$


onde $\{\mathbf{r}\}=\left\{\mathbf{r}_{1}, \ldots, \mathbf{r}_{N}\right\}$ é o conjunto de coordenada espacial de um sistema de $N$ elétrons. O hamiltoniano, $\mathcal{H}(\{\mathbf{r}\}, t)=\mathcal{T}(\{\mathbf{r}\})+\mathcal{W}(\{\mathbf{r}\})+\mathcal{V}(\{\mathbf{r}\}, t)$, consiste na energia cinética,

$$
\mathcal{T}(\{\mathbf{r}\})=-\frac{1}{2} \sum_{i=1}^{N} \nabla_{i}^{2}
$$

na interação elétron-elétron,

$$
\mathcal{W}(\{\mathbf{r}\})=\frac{1}{2} \sum_{i \neq j}^{N} \frac{1}{\left|\mathbf{r}_{i}-\mathbf{r}_{j}\right|}
$$

e num potencial externo,

$$
\mathcal{V}(\{\mathbf{r}\}, t)=\sum_{i=1}^{N} v_{\text {ext }}\left(\mathbf{r}_{i}, t\right)
$$

Runge-Gross demonstraram que existe uma correspondência unívoca entre o potencial externo dependente do tempo $v(\mathbf{r}, t)$ e a densidade eletrônica $\rho(\mathbf{r}, t)$ para sistemas de muitos corpos que evoluam de um estado inicial fixo, este é o teorema central da TD-DFT, também conhecido como teorema de Runge-Gross [77. Este resultado implica que, se a única informação existente sobre o sistema for a sua densidade eletrônica, nós podemos determinar o potencial externo que produz essa densidade. Definido o potencial externo pode-se então resolver a equação de Schrödinger dependente do tempo, e assim obter todas as propriedades do sistema.

De posse do teorema de Runge-Gross é possível construir um esquema de KohnSham dependente do tempo. Este esquema obedecerá a equação de Schrödinger dependente do tempo,

$$
i \frac{\partial}{\partial t} \phi_{i}(\mathbf{r}, t)=h_{K S}(\mathbf{r}, t) \phi_{i}(\mathbf{r}, t)
$$

onde $h_{K S}$ é o hamiltoniano de Kohn-Sham e é definido como:

$$
h_{K S}(\mathbf{r}, t)=-\frac{\nabla^{2}}{2}+v_{K S}[\rho](\mathbf{r}, t)
$$


Por construção, a densidade do sistema interagente pode ser determinada a partir dos orbitais de Kohn-Sham, $\phi_{i}$,

$$
\rho(\mathbf{r}, t)=\sum_{i=1}^{N}\left|\phi_{i}(\mathbf{r}, t)\right|^{2}
$$

Como no esquema Kohn-Sham para o estado excitado, o potencial de Kohn-Sham dependente do tempo é normalmente escrito como a soma de três termos,

$$
v_{K S}(\mathbf{r}, t)=v_{\text {ext }}(\mathbf{r}, t)+v_{\text {Hartree }}[\rho](\mathbf{r}, t)+v_{x c}[\rho](\mathbf{r}, t)
$$

o primeiro termo é o potencial externo, o segundo representa a interação eletrostática entre elétrons,

$$
v_{\text {Hartree }}(\mathbf{r}, t)=\int d^{3} r^{\prime} \frac{\rho(\mathbf{r}, t)}{\left|\mathbf{r}-\mathbf{r}^{\prime}\right|}
$$

O terceiro termo na equação 2.110, o potencial de troca-correlação $x c$, inclui todos os efeitos não triviais de muito corpos, e tem uma dependência funcional, extremamente complexa, com a densidade. Esta dependência é não local, tanto no espaço quanto no tempo, ou seja, o potencial num tempo $t$ e uma posição $\mathbf{r}$ dependerá da densidade em todas as outras posições e em todos os instantes de tempo. O que se faz então é separar do potencial $v_{K S}$ em dois termos bem conhecidos, o potencial externo e o potencial de Hartree, e o potencial $x c$ é geralmente aproximado usando argumentos físicos e matemáticos. Portanto, a qualidade dos resultados obtidos dependerá da qualidade da aproximação usada. Assim como na teoria do funcional da densidade para o estado fundamental, esta é a aproximação fundamental na TD-DFT.

Das várias maneiras de se implementar a DFT dependente do tempo, a que tem tido maior sucesso faz uso da Teoria de Resposta Linear.

A chave nesse processo é a função de resposta linear da densidade $\chi\left(\mathbf{r}, \mathbf{r}^{\prime}, \omega\right)$ que conecta a perturbação externa $\delta v(\mathbf{r}, \omega)$ à perturbação resultante na densidade eletrônica $\delta \rho(\mathbf{r}, \omega)$ através de 


$$
\delta \rho(\mathbf{r}, \omega)=\int \chi\left(\mathbf{r}, \mathbf{r}^{\prime}, \omega\right) \chi\left(\mathbf{r}, \mathbf{r}^{\prime}, \omega\right) d \mathbf{r}^{\prime}
$$

A função resposta da densidade pode ser escrita como 77

$$
\chi\left(\mathbf{r}, \mathbf{r}^{\prime}, \omega\right)=\lim _{\eta \rightarrow 0} \sum_{k}\left[\frac{\langle 0|\rho(\mathbf{r})| k\rangle\left\langle k\left|\rho\left(\mathbf{r}^{\prime}\right)\right| 0\right\rangle}{\omega-\left(E_{k}-E_{0}\right)+i \eta}-\frac{\left\langle 0\left|\rho\left(\mathbf{r}^{\prime}\right)\right| k\right\rangle\langle k|\rho(\mathbf{r})| 0\rangle}{\omega+\left(E_{k}-E_{0}\right)+i \eta}\right]
$$

sendo $\rho$ o operador densidade e os estados $|k\rangle$ de energia $E_{K}$ formam um conjunto completo, $\eta$ é um número infinitésimal positivo. Os polos da função resposta da densidade correspondem às energias de excitação do sistema e os numeradores estão relacionados às correspondentes forças de oscilador. A partir disso chega-se a uma equação de autovalores (não linear) que determina as energias de excitação através dos orbitais de Kohn-Sham.

\subsection{Ajuste das Cargas Parciais - Método Chelpg}

As cargas parciais são de fundamental importância na avaliação de interações intermoleculares em métodos de mecânica molecular, dinâmica molecular e simulações de Monte Carlo, principalmente de moléculas polares. Nesse sentido, o conceito químico de populações eletrônicas atômicas, ou cargas atômicas parciais é muito útil e de grande significância. O conceito de cargas pontuais centradas em átomos implica na aproximação do modelo de cargas pontuais, onde as cargas representam termos monopolares centrados nos átomos em uma expansão multipolar da densidade de carga. Consequentemente, as cargas atômicas fornecem uma representação mais simples possível da densidade de carga de uma molécula.

Ao contrário da densidade eletrônica, as cargas atômicas não podem ser calculadas teoricamente de uma maneira única, pois essas não são valores esperados da função de onda, isto é, não são observáveis. Deste modo, todos os métodos para 
o cálculo teórico de cargas atômicas são invariavelmente arbitrários, resultando, em geral, em uma grande variação dos valores numéricos das cargas calculadas segundo diferentes metodologias.

A comparação dos valores absolutos de cargas atômicas calculadas segundo diferentes métodos ainda carece de significados. Contudo, há alguns critérios mínimos desejáveis para que um conjunto de cargas atômicas calculadas teoricamente sejam satisfatórias:

- A soma das cargas de todos os átomos na molécula deve resultar na carga total da molécula;

- Devem refletir a simetria da molécula e serem invariantes com respeito à rotação e translação desta;

- Devem ser consistentes com as eletronegatividades atômicas e possuir uma interpretação física clara, sendo portanto compatíveis com a intuição química;

- Não devem apresentar uma dependência elevada com o conjunto de base e devem tender a um limite bem definido quando o conjunto de base tende a completeza;

- Devem reproduzir satisfatoriamente o momento dipolar e o potencial eletrostático molecular.

Uma maneira inicilamente utilizada para obter essas cargas é através da análise populacional de Mulliken [78], que é seguramente o método mais tradicional e de uso mais difundido entre os químicos. Esse método baseia-se na teoria dos orbitais moleculares, $\left\{\Psi_{i}\right\}$ é definido por uma combinação linear de $k$ orbitais atômicos, também chamados funções de base, $\left\{\chi_{j}\right\}$, cujos os coeficientes são determinados pelo método Hartree-Fock, 


$$
\Psi_{i}=\sum_{j=1}^{k} C_{j i} \chi_{j}
$$

A análise populacional de Mulliken consiste em associar um número de elétrons a um determinado átomo de acordo com o que todos os orbitais atômicos centrados naquele átomo contribuem para a densidade eletrônica [44]. Subtraindo esse valor do número atômico $(Z)$ do átomo em questão, obtém se carga parcial líquida sobre aquele átomo.

A análise populacional de Mulliken deve sua grande popularidade em grande parte a intensiva aplicação dos métodos que empregam a teoria do orbitais moleculares e, consequentemente à facilidade com que pode ser calculada. Além disso todas as variáveis necessárias para efetuar a análise populacional são obtidas diretamente, não sendo necessário nenhum custo computacional adicional.

O problema com a análise populacional de Mulliken é que ela não é a única maneira de se fazer análise populacional baseada em orbitais atômicos e também apresenta uma forte dependência do conjunto de base empregada no cálculo [79|. Este efeito é geralmente mais pronunciado quando o conjunto de base possue orbitais difusos. Neste caso, a carga originalmente pertencente a um orbital de um determinado átomo pode contribuir para a carga total de um átomo vizinho aumentado artificialmente esta última.

Com o intuito de superar as dificuldades associadas à análise populacional de Mulliken, foi proposta a definição de um modelo de cargas pontuais bastante distinto desse último. Esse modelo baseia-se no cálculo de um conjunto de cargas atômicas pontuais de modo que estas representem o melhor possível o potencial eletrostático quântico em um conjunto de pontos pré definidos ao redor da molécula. Estas cargas atômicas são chamadas derivadas do potencial [80].

Há vários métodos que fazem uso dessa abordagem [81,82, em particular, o 
método CHELPG (Charges from Electrostatic Potential Grid based) 83 tem sido bastante usado. O método consiste no seguinte procedimento: (i) Cria-se uma grade retangular de pontos em torno da molécula. Dessa grade são excluídos todos os pontos que estão dentro das esferas de van der Walls centradas nos átomos das moléculas; (ii) Calcula-se o potencial eletrostático em cada um dos pontos dessa grade; (iii) Ajustamse as cargas parciais nas posições dos núcleos atômicos de modo a reproduzir o potencial eletrostático. O único vínculo adicional no ajuste é que a carga total tem que ser igual a carga líquida da molécula que gerou o potencial.

As cargas derivadas do potencial apresentam a vantagem de, em geral, serem fisicamente mais satisfatórias que as cargas de Mulliken [84|. Como consequência do seu método de cálculo, as cargas derivadas do potencial reproduzem adequadamente o potencial eletrostático molecular, o que é relevante em simulações de interações intermoleculares, obtendo-se uma descrição adequada de interações eletrostáticas entre moléculas. Em geral, o método CHELPG permite a reprodução satisfatória do momento dipolar. Por conta disso esse método será utilizado neste trabalho para gerar as cargas parciais que serão utilizadas na avaliação da interação intermolecular através de simulações com Monte Carlo. 


\section{Capítulo 3}

\section{Métodos para inclusão dos efeitos do solvente.}

\subsection{Introdução}

Nesse capítulo descreveremos alguns dos diferentes métodos que são utilizados no estudo dos efeitos do solvente, desde métodos quânticos a métodos clássicos. Falaremos especificamente dos modelos utilizado nessa tese. O método quântico será discutido em termos do modelo contínuo e o método clássico será discutido a partir de simulações clássicas ustilizando a amostragem de Monte Carlo.

\subsection{Método Quântico: Modelo Contínuo para o Sol- vente}

Nos últimos anos, tratamentos totalmente quanto-mecânicos da interação solutosolvente e solvente-solvente, onde moléculas do solvente interagem explicitamente com o soluto, tem sido introduzido e seu uso vem crescendo rapidamente. Entretanto, isto não elimina a utilidade dos modelos contínuos, cuja maior vantagem é o custo computacional mais baixo. Estes modelos tiveram origem nos trabalhos de Born 85, Kirkwood [86] e Onsager [87]. No modelo contínuo o solvente é tratado como um mate- 
rial dielétrico, caracterizado por parâmetros macroscópicos, principalmente a constante dielétrica $\epsilon$. Todos esses modelos são baseados na equação de Poisson, que relaciona a distribuição de cargas $\rho$, o potencial eletrostático $\phi$ e a constante dielétrica $\epsilon$ :

$$
\nabla^{2} \phi(r)=-\frac{4 \pi \rho(r)}{\epsilon}
$$

No modelo contínuo, o soluto é colocado em uma cavidade $(\epsilon=1)$ circundada por um dielétrico de constante $\epsilon$. Portanto, há duas regiões distintas para as quais a equação de Poisson deve ser resolvida satisfazendo as condições de contorno usuais na superfície da cavidade.

Onsager, introduziu a ideia de campo de reação. Um dielétrico com cavidade esférica de raio $a$, na qual se encontra uma molécula cuja distribuição de cargas é representada pelo momento de dipolo $\mu$, é polarizado pelo campo elétrico desse dipolo. A distribuição de cargas no dielétrico resultante dessa polarização dá origem ao campo de reação,

$$
R=\frac{2(\epsilon-1)}{2(\epsilon+1)} \frac{\vec{\mu}}{a^{3}}
$$

Esse campo reativo é sempre paralelo ao momento de dipolo e, portanto, atuará no soluto aumentando a assimetria na sua distribuição de cargas. Se a molécula for apolar, então não há campo de reação e o campo elétrico sentido pelo soluto deve ser bastante reduzido. A equação (3.2) é uma aproximação que considera apenas o primeiro termo da distribuição de cargas. Implementações mais sofisticadas podem utilizar também campos de reação gerados por quadrupolos, octopolos, etc [88].

Um dos pontos a favor do modelo contínuo é a possibilidade em se fazer um tratamento puramente quântico da interação soluto-solvente, pois tratam do solvente sem aumentar o número de elétrons explícitos. Assim, o potencial de interação contém um termo adicional que depende do campo de reação. Incorporando esse termo no 
hamiltoniano e usando a aproximação de Hartree-Fock, obtém-se:

$$
\left\{F_{i}-\left[\frac{2(\epsilon-1)}{2(\epsilon+1)}\right] \frac{1}{a^{3}}\langle\Psi|\mu| \Psi\rangle\right\} \chi_{i}=e_{i} \chi_{i}
$$

sendo $\chi_{i}$ orbitais moleculares. Há um termo na equação (3.3) que depende do momento de dipolo do soluto (e possivelmente de outros momentos), que por sua vez depende da função de onda total $\Psi$, criando um ciclo adicional de autoconsistência. Por isso, tal procedimento é comumente referido por Campo de Reação Autoconsistente (SCRF).

Outro ponto favorável aos modelos contínuos é que a perturbação provocada pelo solvente, a longa distâncias, pode ser bem representada por uma distribuição de cargas, entretanto, para a estrutura microscópica do solvente nas proximidades do soluto, essa distribuição determinada por parâmetros macroscópicos é um simplificação exagerada não sendo possível obter, através desse modelo, uma descrição adequada das ligações de hidrogênio, por exemplo. Além disso, os resultados obtidos com os modelos contínuos apresentam uma dependência com o tamanho da cavidade, o que pode ser entendido olhando-se a expressão do campo de reação, equação (3.2), em que o campo depende inversamente do cubo do raio da cavidade, sendo assim bastante sensível ao mesmo.

A forma da cavidade também é um problema pois poucas moléculas têm uma forma que possa ser considerada esférica ou elipsoidal. Isto pode ser resolvido fazendo com que a cavidade seja formada pela união de esferas centradas nos átomos do soluto. Dessa forma, a cavidade tem necessariamente o formato da molécula de referência, sendo que o tamanho das esferas deve estar sujeito a algum tipo de ajuste.

O método mais popular dentre aqueles que utilizam esse tipo de cavidade é o modelo contínuo polarizável (PCM), introduzido por Tomasi e colaboradores 89, 90. Eles utilizam os raios de van der Waals acrescidos de aproximadamente $20 \%$ para desenhar a cavidade. Esse modelo tem sido bastante utilizado e com relativo sucesso, principalmente para análise de propriedades que dependam pouco de interações específicas entre soluto e solvente. Esse modelo é o que será considerado aqui quando 
tratarmos o solvente como um meio contínuo.

\subsection{Métodos Clássicos}

São métodos em que o sistema solvatado é descrito através de conceitos clássicos. Uma vantagem dessa aproximação é o baixo custo computacional, o que possibilita a investigação de sistemas maiores.

\subsubsection{Métodos de simulação}

A utilização de métodos específicos para estudar sistemas líquidos está associada à complexidade das interações intermoleculares e à ausência de simetria espacial que caracterizam esses sistemas. Nesse âmbito, os modelos moleculares para o estudo de líquidos com simulação molecular podem levar em conta características e propriedades das moléculas que constituem o sistema, e o modo como estas interagem.

Na mecânica estatística de equilíbrio todas as propriedades estruturais e termodinâmicas de um sistema de partículas podem, em princípio, ser determinadas se for conhecido o potencial de interação entre as partículas. Assumindo então que o potencial de interação $U(r)$ é conhecido para todas as partículas, temos que o hamiltoniano $\mathcal{H}$ para um sistema de $\mathrm{N}$ partículas será dado por [39]:

$$
\mathcal{H}=\mathcal{H}(\mathbf{r}, \mathbf{p})=\sum_{i=1}^{N} \frac{p_{i}^{2}}{2 m_{i}}+U(r)
$$

onde o primeiro termo é a energia cinética do sistema e o segundo termo é o potencial de interação, $\mathbf{r}$ é a coordenada coletiva representando todas as partículas, $\mathbf{r}=\left(\mathbf{r}_{1}, \mathbf{r}_{2}, \ldots, \mathbf{r}_{\mathbf{N}}\right)$ e $\mathbf{p}$ é o conjunto dos momentos lineares de todas as partículas.

Assumindo que o sistema esteja em contato com um reservatório térmico infinitamente grande, com temperatura absoluta $T$ e que o número de partículas $N$ e o 
volume $V$ são conhecidos (ensemble $N V T$ [91]), a grandeza que faz a conexão entre o mundo microscópico (mecânica estatística) e o macroscópico (termodinâmica), no limite termodinâmico, é a função de partição canônica $Z$, esta função raramente pode ser obtida exatamente, exceto para sistemas ou problemas-modelo simples.

A função de partição é definida por:

$$
Z(N, V, T)=\iint e^{-\beta \mathcal{H}(\mathbf{r}, \mathbf{p})} d \mathbf{p} d \mathbf{r}
$$

onde $\mathcal{H}$ é o hamiltoniano dado pela equação (3.4) e $\beta=1 / k_{B} T$, $k_{B}$ é a constante de Boltzmann $\left(k_{B}=1,38 \cdot 10^{23} \mathrm{~J} / K\right)$.

Assim como o hamiltoniano, a função de partição $Z$ também é separável nas suas partes cinética e potencial, também chamada configuracional,

$$
\begin{aligned}
Z(N, V, T) & =Z_{K} \cdot Z_{\text {conf }} \\
Z_{K} & =\int \exp \left(-\beta \sum_{i=1}^{N} \frac{p_{i}^{2}}{2 m_{i}}\right) d \mathbf{p} \\
Z_{\text {conf }} & =\int \exp (-\beta U(\mathbf{r})) d \mathbf{r}
\end{aligned}
$$

No formalismo da mecânica estatística 92,93 , uma propriedade termodinâmica observável é uma média no ensemble de estados microscópicos de um sistema, e a escolha apropriada de um ensemble está associada aos vínculos que são impostos ao sistema em estudo. Os sistemas comumente estudados em simulação de líquidos estão sob as condições do ensemble canônico $(N V T)$ e do ensemble isobárico-isotérmico (NPT), em que o número de partículas $N$, a pressão $P$ e a temperatura $T$ são mantidos fixos. O valor médio de uma grandeza $W(\mathbf{r}, \mathbf{p})$ no ensemble $N V T$, por exemplo, é dada por: 


$$
\langle W\rangle_{N V T}=\frac{1}{Z} \int W(\mathbf{r}) e^{-\beta \mathcal{H}(\mathbf{r}, \mathbf{p})} d \mathbf{r} d \mathbf{p}
$$

onde a média é feita em cima do ensemble de configurações.

Neste trabalho as simulações clássicas foram realizadas no sentido de gerar configurações acessíveis ao sistema solvatado (Soluto + Solvente). Cada configuração possui informações acerca das coordenadas de todas as moléculas. Um ponto importante na geração dessas configurações é a definição dos potenciais intermoleculares que descrevem a interação soluto-solvente, sendo estes cruciais para uma adequada descrição das relações entre propriedades moleculares e características observadas experimentalmente. Há duas maneiras de se obter as configurações acessíveis. Uma delas é através do método de Dinâmica Molecular (DM) [94]. Nesse método, a partir do potencial de interação $U(\mathbf{r})$, as forças que atuam sobre os átomos são calculadas, $\mathbf{F}=-d \mathbf{U} / d \mathbf{r}$, e as equações de movimento são resolvidas para um intervalo de tempo, $\delta t$. Assim as configurações do sistema são geradas de forma determinística. A outra maneira é através do método Monte Carlo (MC) em que as configurações são geradas por processos estocásticos e não há como determinar a ordem temporal dessas configurações geradas.

Neste trabalho as configurações foram geradas através do método Monte Carlo, pois não havia interesse em descrever propriedades dinâmicas do sistema. O seu uso reduz considerávelmente o custo computacional quando comparado com a Dinâmica Molecular. Vale salientar que todo desenvolvimento teórico feito sobre as simulações computacionais é baseado na hipótese de que tanto o processo determinístico quanto o estocástico, para gerar as configurações, são ergódicos 95$]$. 


\subsubsection{Método Monte Carlo}

O método Monte Carlo foi desenvolvido por von Neumann, Ulam e Metropoplis para estudar a difusão de nêutrons em materiais que poderiam sofrer fusão. Porém, desde o início do século XIX já se fazia uso do método de amostragem para estudar alguns problemas. Esses métodos de amostragem envolvem a geração de números randômicos que depois são usados para uma série de operações aritméticas e lógicas, que serão sempre as mesmas a cada passo da simulação. Essas operações são realizadas com sucesso pelos computadores e portanto, o desenvolvimento da técnica está intimamente ligada ao desenvolvimento dessas máquinas.

O método Monte Carlo é comumente usado para calcular numericamente as integrais envolvidas na equação $(3.9)$, aproximando-as por somas sobre um número $n$ de pontos escolhidos aleatoriamente no espaço de fase, de modo que podemos escrever,

$$
\langle W\rangle=\frac{\sum_{n=1}^{N} W(\mathbf{r}) e^{-\beta U(\mathbf{r})}}{\sum_{n=1}^{N} e^{-\beta U(\mathbf{r})}}
$$

Entretanto, esse método de amostragem aleatória não é muito útil para grande maioria dos problemas de interesse em que o número $n$ de observações é muito grande no espaço multiconfiguracional. Ainda, para $N$ moléculas na densidade do líquido, uma amostragem aleatória dos estados levaria a grande proximidade ou mesmo superposição de duas moléculas, essas configurações, por sua vez, resultam em uma energia alta do sistema e, portanto, uma probabilidade baixa de ocorrência. Estas configurações devem ser evitadas.

Um procedimento mais eficiente para a seleção das configurações é restrito às regiões mais densas do espaço de fase e termodinamicamente mais prováveis. O algoritmo de amostragem modificado para o método MC convencional, proposto por Metropolis e colaboradores [96], é denominado importance sampling e consiste em evitar a amostragem excessiva de configurações que tem pequena contribuições para o 
cálculo da média.

Na proposta de Metropolis a idéia é amostrar configurações a partir de uma distribuição $\rho(r)$ transformando a soma da equação $(3.10) \mathrm{em}$

$$
\langle W\rangle=\frac{\frac{1}{\rho(r)} \sum_{n=1}^{N} W(\mathbf{r}) e^{-\beta U(\mathbf{r})}}{\frac{1}{\rho(r)} \sum_{n=1}^{N} e^{-\beta U(\mathbf{r})}}
$$

que representa a soma com um peso dado pelo termo $\rho(r)$. No MC Metropolis esta seleção é feita levando em conta os fatores de Boltzamann, $\rho(r)=\rho(r)_{N V T}=e^{-\beta U(r)}$. Usando esta distribuição de probabilidades na equação (3.11), obtemos:

$$
\langle W\rangle_{N V T}=\frac{1}{N} \sum_{n=1}^{N} W(r(n))
$$

onde $r(n)$ é o conjunto de coordenadas $r=\left(r_{1}^{n}, r_{2}^{n}, r_{3}^{n}, \ldots\right)$ da configuração específica $n$.

Na equação (3.12) $W(r(n))$ é o valor de $W(r)$ nas configurações geradas pela simulação. Como as configurações são geradas com o peso de Boltzmann a média $\langle W\rangle$ é uma média simples da grandeza $W(r)$. Os estados que não são estatisticamente representativos são filtrados pela amostragem de Metropolis.

No algoritmo de Metropolis, a transição de uma configuração $\Gamma_{i}$ para uma configuração $\Gamma_{i+1}$ é obtida através de um processo probabilístico denominado cadeia de Markov [94], que é construída de tal maneira que esta cadeia tenha uma distribuição limite igual a função de partição ou a função de distribuição desejada. Uma cadeia de Markov é uma sequência de tentativas que deve satisfazer duas condições:

1. O resultado de cada tentativa deve pertencer a um conjuto finito de resultados, $\left\{\Gamma_{1}, \Gamma_{2}, \Gamma_{3}, \ldots\right\}$, chamado de espaço de estados;

2. O resultado de cada tentativa deve depender somente do resultado da tentativa imediatamente anterior. 
Dois estados $\Gamma_{m}$ e $\Gamma_{n}$ são ligados por uma taxa de transição $\pi_{m n}$, que é a probabilidade para o sistema sair do estado $m$ para o estado $n$. As probabilidades de transição formam uma matriz que é chamada matriz de transição, essa matriz é irredutível, isto é, a partir de um estado é possível acessar qualquer outro estado do sistema. Isto também quer dizer que a cadeia é ergódica.

Portanto, seja $\rho_{m}$ a distribuição de probabilidade do estado $\Gamma_{m}$ através da matriz de transição $\pi_{m n}$ podemos obter $\rho_{n}$, que é a distribuição para o estado $\Gamma_{n}$. A matriz de transição deve obdecer a seguinte equação de autovalores com autovalor 1 ,

$$
\rho_{n}=\sum_{m} \rho_{m} \pi_{m n} \quad(m \neq n)
$$

$\pi$ é uma matriz estocástica desde que:

$$
\pi_{m n}>0 \quad \text { e } \quad \sum_{n} \pi_{m n}=1
$$

essa é uma matriz de transição para uma cadeia de Markov.

No caso de um sistema líquido, é preciso construir uma matriz de transição muito grande, que seja estocástica e ergódica. Nesse caso os elementos dessa matriz são desconhecidos, mas os elementos do vetor da distribuição limite da cadeia são dados pelos valores da função de partição em cada estado do sistema, ou seja, $\rho_{m}=\rho_{N V T}\left(\Gamma_{m}\right)$ para cada estado $\Gamma_{m}$.

Uma técnica útil para resolver a equação (3.13 é substituir essa equação por uma condição desnecessária, porém forte, chamada de reversibilidade microscópica (ou balanço detalhado):

$$
\rho_{m} \pi_{m n}=\rho_{n} \pi_{n m}
$$

Nessa condição de reversibilidade microscópica, os dois estados estão ligados por 
uma taxa de transição, onde a probabilidade do sistema sair do estado $m$ e ir para o estado $n$ é a mesma para o sistema sair do estado $n$ e ir para o estado $m$.

Agora, somando sobre todos os estados $m$ na equação (3.15) e usando a equação (3.14) nós podemos reescrever a equação 3.13),

$$
\begin{aligned}
\sum_{m} \rho_{m} \pi_{m n} & =\sum_{m} \rho_{n} \pi_{n m} \\
& =\rho_{n} \sum_{m} \pi_{n m} \\
& =\rho_{n}
\end{aligned}
$$

Uma maneira adequada para construir uma trajetória no espaço de fase usando o ensemble canônico envolve a escolha de uma matriz de transição que satisfaça as condições de ser estocástica, equação (3.14), e a de ser reversível microscopicamente, equação (3.15). O primeiro arranjo feito desse modo foi sugerido por Metropolis e colaboradores em 1953 [96] e é comumente conhecido como solução assimétrica. Se temos dois estados $m$ e $n$ com probabilidades $\rho_{m}$ e $\rho_{n}$, respectivamente, esta solução assimétrica considera dois casos:

$$
\begin{array}{lll}
\pi_{m n}=\alpha_{m n} & \rho_{n} \geq \rho_{m} & m \neq n \\
\pi_{m n}=\alpha_{m n}\left(\rho_{n} / \rho_{m}\right) & \rho_{n}<\rho_{m} & m \neq n
\end{array}
$$

onde para o ensemble canônico, $\rho_{n}=\rho_{N V T}\left(\Gamma_{n}\right)=e^{-\beta U\left(r_{n}\right)}$ e $\rho_{m}=\rho_{N V T}\left(\Gamma_{m}\right)=$ $e^{-\beta U\left(r_{m}\right)}$. Sendo também importante permitir a possibilidade de que o sistema líquido permaneça no mesmo estado, ou seja,

$$
\pi_{m m}=1-\sum_{n \neq m} \pi_{m n}
$$


$\mathrm{Na}$ solução proposta por Metropolis, $\alpha$ é uma matriz estocástica simétrica, $\left(\alpha_{m n}=\alpha_{n m}\right)$, e essa simetria garante que a solução obedeça à reversibilidade microscópica. Para implementar a solução de Metropolis na matriz de transição é preciso especificar a matriz $\alpha$. Essa matriz é responsável por levar o sistema do estado $m$ para qualquer um dos estados vizinhos $n$ com a mesma probabilidade. Existe muita liberdade na escolha da matriz $\alpha$ e a única restrição é de que esta seja simétrica.

Na prática, para um simulação no ensemble $N V T$, temos que se uma molécula é movida para uma nova posição e a energia do sistema diminui, $\left(U_{F}<U_{I}\right)$ então esta nova posição é aceita; porém se a nova posição provoca um aumento na energia $\left(U_{F}>U_{I}\right)$ então um número aleatório $\gamma$ entre 0 e 1 é gerado e se este número for menor ou igual à probabilidade de transição $\pi_{F, I}=\rho_{F} / \rho_{I}=e^{-\left(U_{F}-U_{I}\right) / k_{B} T}=e^{-\Delta U / k_{B} T}$, a nova posição é aceita, apesar da energia ter aumentado, caso contrário $\left(\gamma>e^{-\Delta U / k_{B} T}\right)$ a nova posição é rejeitada.

Esta solução proposta por Metropolis não é única, porém é uma forma muito simples de gerar uma cadeia de Markov.

Para tratarmos a proposta de Metropolis no ensemble isotérmico-isobárico, $(N P T)$, devemos apenas usar a distribuição de probabilidades que caracterizam esse ensemble, ou seja, $\rho_{m}=\rho_{N P T}\left(\Gamma_{m}\right)$ onde

$$
\rho_{N p T}(r)=e^{-\beta[U(r)+p V]}
$$

Esse ensemble foi pensado como sendo particularmente apropriado para simular misturas já que medidas experimentais são feitas à pressão constante e teorias de misturas são sempre formuladas com essa suposição. O cálculo da média de uma grandeza usando o ensemble NPT será dado por:

$$
\langle W\rangle_{N P T}=\frac{\int W(\mathbf{r}) e^{-\beta[U(\mathbf{r})+P V]} d \mathbf{r} d V}{\int e^{-\beta[U(\mathbf{r})+P V]}}
$$


A dificuldade nesta expressão é que o volume além de está explícito na probabilidade do ensemble, está também presente implicitamente no domínio de integração das coordenadas das partículas, tornando difícil o seu controle. Sendo assim, é mais interessante desvincular o domínio de $U(\mathbf{r})$ do volume [97]. Isto é feito tornando adimensionais as coordenadas das partículas do sistema, através da transformação:

$$
\mathbf{r}_{i} \rightarrow\left(L_{x} q_{i x}, L_{y} q_{i y}, L_{z} q_{i z}\right)
$$

onde $\mathbf{r}_{i}$ representa as coordenadas da partícula $i$ e $\mathbf{q}_{i}=\left(q_{i x}, q_{i y}, q_{i z}\right)$. O domínio da coordenada $q_{i j}$, com $i=1,2,3, \ldots, N$ e $j=x, y, z$, é o intervalo [0,1], onde esse intervalo vai depender das coordenadas dos vértices da caixa que contém o volume. Com essa transformação a equação (3.23) passa a ser escrita como:

$$
\langle W\rangle_{N P T}=\frac{\int W(\mathbf{Q}) e^{-\beta[U(\mathbf{Q})+P V]} V^{N} d \mathbf{Q} d V}{\int e^{-\beta[U(\mathbf{Q})+P V]}}
$$

$\operatorname{com} \mathbf{Q}=\left(\mathbf{q}_{1}, \mathbf{q}_{2}, \ldots, \mathbf{q}_{N}\right)$. A distribuição de probabilidades na equação 3.22 pode ser reescrita como:

$$
\rho_{N p T}(r)=e^{-\beta[U(Q)+P V]+N \ln V}
$$

Portanto, substituindo a densidade de probabilidade $\rho_{N P T}$ dada pela equação (3.26) no conjunto de equações (3.20) e (3.21) nós teremos uma solução analoga à proposta de Metropolis para o ensemble $N V T$. A mudança na forma da distribuição de probabilidade para o ensemble $N P T$ é interessante porque torna a variação no volume independente da mudança nas coordenadas do sistema que são relativas ao volume corrente da simulação. 


\subsubsection{Detalhes da Implementação}

Em nosso trabalho, o estudo do sistema solvatado foi feito usando o método Monte Carlo tal como implementado no programa DICE 98 cuja implementação segue da seguinte forma :

1. Iniciamos a simulação com uma configuração inicial qualquer de um sistema com $N$ moléculas confinadas em uma caixa de volume $V$ a uma temperatura T. A partir daí replica-se a caixa em todas as direções, impondo condições de contorno periódicas, para eliminar os efeitos de borda. Este método consiste em replicar a caixa com o sistema original em todas as direções, fazendo com que as moléculas da caixa original não interajam mais com as paredes, mas sim com outras moléculas que são réplicas das existentes na caixa original. Há também a introdução de um raio de corte $r_{c}$, significando que cada molécula só interage com outras que estão separadas por uma distância menor que o raio de corte.

2. Uma nova configuração é obtida partindo da configuração inicial, isso ocorre através de uma translação de $\delta_{r}=\left(\delta_{x}, \delta_{y}, \delta_{z}\right)$ e rotação de $\delta \Theta$, num eixo sorteado, de alguma molécula sorteada. A translação é feita calculando-se um novo valor para as coordenadas $(x, y, z)$ do centro de massa da molécula através das equações:

$$
\begin{aligned}
& x_{i+1}=x_{i}+a \gamma_{1} \\
& y_{i+1}=y_{i}+a \gamma_{2} \\
& z_{i+1}=z_{i}+a \gamma_{3}
\end{aligned}
$$

onde $\gamma_{i}$ é um número aleatório entre -1 e 1 e $a$ o deslocamento máximo, que pode ser ajustado ao longo da simulação.

A rotação é feita sorteando-se um eixo aleatório $\gamma_{4}$ dentre os eixos $x, y, z$ e um ângulo para rotacionar a molécula através da equação: 


$$
\phi_{i+1}=\phi_{i}+b \gamma_{4}
$$

sendo $b$ o ângulo máximo de rotação.

Para o tratamento no ensemble $N P T$ há também a mudança no volume, e esta mudança se dá obedecendo as seguintes equações:

$$
\begin{aligned}
& L_{i+1, x}=L_{i x}+c \gamma_{6} \\
& L_{i+1, y}=L_{i y}+c \gamma_{6} \\
& L_{i+1, z}=L_{i z}+c \gamma_{6}
\end{aligned}
$$

sendo $c$ a variação máxima dos lados da caixa, este parâmetro também pode ser ajustado durante a simulação.

Este movimento de translação e rotação é feito para todas as moléculas do sistema e em cada uma é aplicada o teste de aceitação.

3. Nem todos os movimentos moleculares são aceitos. É a técnica de amostragem que estabelece as regras de aceitação, ou rejeição dos movimentos. Neste programa é usada a técnica de amostragem de Metropolis, que foi detalhada na seção 3.2, essa técnica usa a variação de energia entre as configurações como termo decisivo na regra de aceitação de uma nova configuração.

4. Para o cálculo das propriedades termodinâmicas são usadas médias e flutuações de algumas grandezas obtidas durante a simulação, são elas: a energia $U$, o primeiro virial $W=-1(1 / 3) r(\partial U / \partial r)$ e o segundo virial $\Theta=(1 / 9) r(\partial(r \partial U / \partial r) / \partial r)$; acumula-se esses valores para a média e volta-se para a etapa 2 do processo. Cada vez que se chega a este ponto, dizemos que foi realizado um passo de Monte Carlo. 
Ao final da simulação, há uma cadeia de configurações que descrevem a evolução do sistema. Em geral, esta evolução pode ser dividida em dois estágios. O primeiro é um estágio não estacionário, termalização, e o segundo é um estágio estacionário, conhecido como estágio de equilíbrio ou estágio de médias. Estes dois estágios podem ser facilmente observados no comportamento da energia do sistema durante a simulação, pois o sistema sai da energia inicial $U_{0}$ e gradualmente atinge um valor de energia médio $\langle U\rangle$, onde fica flutuando. Para o cálculo das propriedades estruturais e termodinâmicas somente as configurações geradas no equilíbrio são consideradas.

\subsubsection{Potencial de Interação Intermolecular}

Uma boa descrição de um sistema molecular está intimamente ligada a escolha do potencial de interação $U(\mathbf{r})$, esse potencial pode ser tratado como uma soma da energia intramolecular de cada molécula e a energia intermolecular. Para modelos de moléculas rígidas, onde a distância e os ângulos das ligações são mantidos fixos, a energia interna é constante durante todo o processo de geração das configurações. Portanto, a representação usada para a energia de uma configuração, nesses sistemas, consiste somente da contribuição intermolecular desprezando-se assim as contribuições intramoleculares devido a rotações e vibrações internas da molécula.

O potencial intermolecular pode ser escrito como:

$$
U(\mathbf{r})=\sum_{i} u_{1}\left(\mathbf{r}_{i}\right)+\sum_{i} \sum_{j>i} u_{2}\left(\mathbf{r}_{i}, \mathbf{r}_{j}\right)+\sum_{i} \sum_{j>i} \sum_{k>j} u_{3}\left(\mathbf{r}_{i}, \mathbf{r}_{j}, \mathbf{r}_{k}\right)+\ldots
$$

sendo $u_{1}$ o potencial que atua em um corpo e representa o efeito sobre o sistema de forças externas; o segundo termo, $u_{2}$, é o potencial de pares e representa a interação entre dois corpos, $u_{2}=u_{2}\left(r_{i j}\right)$ onde $r_{i j}=\left|\mathbf{r}_{i}-\mathbf{r}_{j}\right| ;$ o terceiro termo, $u_{3}$, representa a interação entre três corpos; o termo seguinte irá representar a interação entre quatro corpos e assim sucessivamente. O que se observa é que, em geral, os termos de in- 
teração entre quatro corpos e ordens seguintes são pequenos comparados aos termos $u_{2}$ e $u_{3}$, potanto, o potencial de interação é truncado em $u_{3}$. Em muitos sistemas a contribuição de três corpos é relevante [94,100, entretanto para sistemas sem orientação preferêncial, como acontece em geral com os líquidos, esta interação já é bastante pequena de tal forma que, para modelar esses sistemas, o potencial é truncado no segundo termo e o potencial de pares é substituido por um potencial efetivo de pares que inclui a contribuição de muitos corpos.

$$
U(\mathbf{r})=\sum_{i} u_{1}\left(\mathbf{r}_{i}\right)+\sum_{i} \sum_{j>i} u_{2}^{e f f}\left(r_{i j}\right)
$$

Uma consequência desta aproximação é que o potencial efetivo de pares tem que reproduzir dados experimentais. Aliás, é através desses dados experimentais e também de resultados de estudos ab initio que se constrói o potencial efetivo.

Considerando sistemas em que não atuam forças externa, $u_{1}=0$, o potencial de interação será dado por:

$$
U(\mathbf{r})=\sum_{i} \sum_{j>i} u_{2}^{e f f}\left(r_{i j}\right)
$$

Em geral, esse potencial efetivo é escrito como uma soma de potenciais de pares entre os sítios das moléculas, esses sítios, em sua maioria, são representados pelos átomos das moléculas. Nesse caso, a interação entre duas moléculas $a$ e $b$ é descrita por:

$$
U_{a b}=\sum_{i}^{e m} \sum_{j}^{a}{ }^{e m b} U\left(r_{i j}\right)
$$

onde $i$ são os sítios da molécula $a, j$ os sítios da molécula $b$ e $r_{i j}$ a distância entre os sítios $i$ e $j$. Na figura (3.1) ilustramos a interação entre dois sítios de duas moléculas.

A energia potencial intermolecular é então reduzida a uma grande soma de po- 


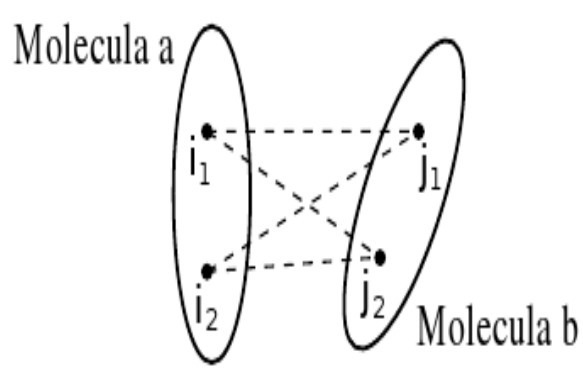

Figura 3.1: Ilustração da interação entre os sítios $i$ da molécula $a$ e os sítios $j$ da molécula $b$. Figura retirada da referência 99

tenciais de interação de pares sítio-sítio. Como não se conhece a expressão formal para $U\left(r_{i j}\right)$, há várias maneiras de escrevê-lo analiticamente. O potencial, $U\left(r_{i j}\right)$, mais usado para tratar sistemas líquidos é o potencial de Lennard-Jones [101] com um termo adicional coulombiano, equação (3.34).

$$
U\left(r_{i j}\right)=4 \epsilon_{i j}\left[\left(\frac{\sigma_{i j}}{r_{i j}}\right)^{12}-\left(\frac{\sigma_{i j}}{r_{i j}}\right)^{6}\right]+\frac{q_{i} q_{j}}{r_{i j}}
$$

Aqui, cada sítio é representado por dois parâmetros $\left(\epsilon_{i}\right.$ e $\left.\sigma_{i}\right)$ e uma carga $q_{i}$. Na equação (3.34), $\epsilon_{i j}=\left(\epsilon_{i} \epsilon_{j}\right)^{1 / 2}$ e $\sigma_{i j}=\left(\sigma_{i} \sigma_{j}\right)^{1 / 2}$. A carga $q_{i}$ é responsável pela descrição dos termos eletrostáticos. Os parâmetros $\epsilon_{i j}$ e $\sigma_{i j}$ podem ser interpretados como sendo a energia de ligação e a distância entre os sítios, respectivamente.

\subsubsection{Propriedades Estruturais: Função de Distribuição Radial}

Para estudarmos as propriedades estruturais, comumente é usada a função de distribuição radial (RDF) de pares, $G(r)|94|$. As funções de distribuição radial podem ser determinadas a partir de técnicas de difração de raios-X, espalhamentos de nêutrons e difração de elétrons. Esta função nos dá a probabilidade de encontrarmos um par de átomos a uma distância $r$, relativa a probabilidade esperada para um sistema de mesma densidade e uma distribuição totalmente aleatória, conhecida como distribuição 
de gás ideal.

Para um sistema de átomos idênticos a RDF deve ser calculada como uma média sobre os pares de átomos indistinguíveis. Numa simulação, a RDF entre átomos de tipo $i$ e átomos do tipo $j, G_{i j}$, é calculada através do histograma de distâncias dos pares de átomos $i$ e $j$ :

$$
G_{i j}\left(r+\frac{1}{2} d r\right)=\frac{n_{i j}(r, r+d r)}{n^{i d}(r, r+d r)}
$$

sendo $n_{i j}(r, r+d r)$ o número de pares $i j$ que estão separados por uma distância entre $r$ e $r+d r$ e $n^{i d}$ é o número de pares equivalente num gás ideal de mesma densidade,

$$
n^{i d}(r, r+d r)=\frac{4 \pi}{3} \rho\left[(r+d r)^{3}-r^{3}\right]
$$

Na figura (3.2) ilustramos a estrutura de um líquido atômico e a representação de sua estrutura pela função de distribuição radial. Os picos da figura (3.2) definem as camadas de solvatação.

Integrando $G_{i j}(r)$ numa casca esférica, podemos analisar como o número de moléculas se distribuem radialmente em torno de outra,

$$
N_{s}(r)=4 \pi \frac{N}{V} \int_{0}^{r} G_{i j}(r) r^{2} d r
$$

O número de moléculas que resulta da integração do primeiro pico é conhecido como número de coordenação e é utilizado para definir a primeira camada de solvatação. A segunda e terceira camada estão associadas as integrações do segundo e terceiro pico da Gij, respectivamente. 


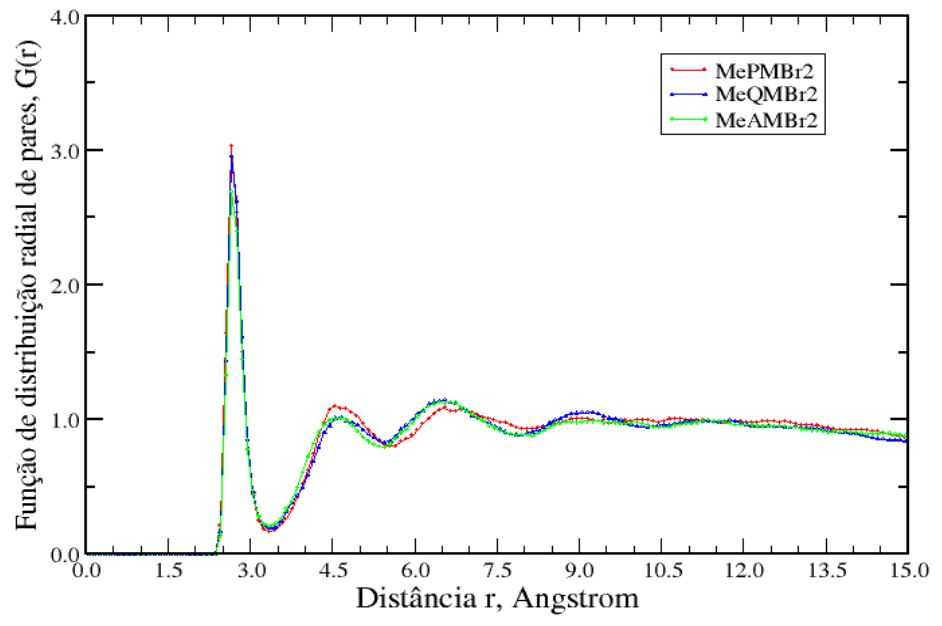

Figura 3.2: Ilustração da representação dos picos da $G(r)$ correspondente. Curva correspondete à $G(r)_{O-O}$, para as sondas solvatadas em água. 


\section{Capítulo 4}

\section{Metodologia experimental}

Neste capítulo apresentaremos as informações sobre os materiais, as medidas experimentais e os equipamentos utilizados para a sua realização neste trabalho.

\subsection{Materiais}

O composto Merocianina de Brooker $\left(C_{14} H 13 N O\right.$, figura 1.1), foi doado pelo professor Omar Abou El Seoud do Departamento de Química Fundamental-Instituto de Química-USP. Os compostos: ácido clorídrico $(\mathrm{HCl})$ e hidróxido de sódio $(\mathrm{NaOH})$ foram comprados da Sigma-Aldrich Co. (St. Louis, MO, USA). Os solventes orgânicos utilizados: metanol $\left(\mathrm{CH}_{3} \mathrm{OH}\right)$, etanol $\left(\mathrm{C}_{2} \mathrm{H}_{6} \mathrm{O}\right)$, DMSO $\left(\mathrm{C}_{2} \mathrm{H}_{6} \mathrm{OS}\right)$, acetonitrila $\left(\mathrm{C}_{2} \mathrm{H}_{3} \mathrm{~N}\right)$ e clorofórmio $\left(\mathrm{CHCl}_{3}\right)$, foram comprados da Sigma-Aldrich Co. (St. Louis, MO, USA). A água $\left(\mathrm{H}_{2} \mathrm{O}\right)$ utilizada foi padrão Milli-Q obtida através do instrumento Milli-Q Plus da Millipore com um $p H \sim 6.5$ 


\subsection{Preparação das amostras}

\subsubsection{Preparação da Merocianina de Brooker em solução}

Foi preparada uma solução estoque com concentração de $10 \mathrm{mM}$ de Merocianina de Brooker (MB) numa mistura de etanol/metanol à razão de 4:1 v/v. Alíquotas dessa solução estoque foram separadas em frascos de vidros, em seguida foi feito a secagem para evaporação do solvente à temperatura ambiente sobre pressão reduzida. Procedimento para secagem: a amostra foi colocada em contato com um fluxo de nitrogênio $\left(N_{2}\right)$ com 99,999\% de pureza e, em seguida, foi colocada sob baixa pressão por aproximdamente 2 horas para remover os resíduos do solvente orgânico. Após a secagem, solventes puros foram adicionados aos frascos contendo a sonda numa concentração de $0.015 \mathrm{mM}$. Analisando os espectros da MB nos solventes e comparando com os dados da literatura, identificamos a presença da forma neutra e protonada $\left(\mathrm{MBH}^{+}\right)$na solução estoque. Sendo assim, preparamos adicionalmente duas outras soluções estoque:a primeira uma solução estoque de metanol ácido com excesso de $\mathrm{HCl}\left(\mathrm{pH}\right.$ ácido, $\mathrm{pH}_{a p}=0.0$ ) e a segunda uma solução estoque de metanol alcalino com excesso de $\mathrm{NaOH}$ ( $\mathrm{pH}$ alcalino, $\mathrm{pH}_{a p}=13.5$ ). Esse procedimento foi realizado para garantirmos a presença de uma única forma na amostra, protonada na solução ácida e neutra na solução alcalina. Essas duas soluções estoque, ácida e alcalina, foram submetidas ao mesmo procedimento de secagem e adição de solventes puros.

\subsubsection{Procedimento de titulação com espectro UV-vis}

Alíquotas de $3 \mu \mathrm{L}$ foram retiradas da solução estoque pura de modo a fornecer soluções com concentração de $0.015 \mathrm{mM}$ nos diferentes pHs tanto em água como em metanol.

Procedimento em água: Foi preparada uma solução ácida com $p H \sim 1.3$ obtida pela adição de $\mathrm{HCl}$ resultando numa solução de hidroclorídrico com água na 
proporção de 1:500 em v:v, e uma solução alcalina com $p H \sim 12.3$ obtida pela adição de $\mathrm{NaOH}$ na concentração de $17 \mathrm{mM}$. Variamos o pH dessas duas soluções titulandoas. Partindo da solução ácida $(p H \sim 1.3)$, o processo de titulação consistiu na adição de alíquotas de solução alcalina, provocando um aumento no pH da solução. Para cada adição da solução alcalina nós medimos o pH da solução com o pHmetro.

Procedimento em metanol: Foi preparada uma solução ácida com $p H_{a p} \sim 0.0$ obtida pela adição de $\mathrm{HCl}$ resultando numa solução de hidroclorídrico com metanol na proporção de 1:500 em v:v, e uma solução alcalina com $p H_{a p} \sim 12.4$ obtida pela adição de $\mathrm{NaOH}$ na concentração de $17 \mathrm{mM}$. Variamos o pH dessas duas soluções titulandoas. Partindo da solução ácida $\left(p H_{a p} \sim 0.0\right)$, o processo de titulação consistiu na adição de alíquotas de solução alcalina, provocando um aumento no pH da solução. Para cada adição da solução alcalina nós medimos o pH da solução com o pHmetro.

As medidas de $\mathrm{pH}$ das amostras foram realizadas utilizando o pHmetro Metter Toledo. Para a solução em metanol, as medidas foram realizadas numa escala de pH aparente, em que o pHmetro é calibrado para medidas em água.

As medidas de absorção eletrônica foram realizadas utilizando um espectofotômetro Cary50. As amostras foram colocadas numa cubeta de quartzo de caminho óptico de $10 \mathrm{~mm}$. As medidas foram realizadas em temperatura ambiente. Em todas as medidas, a absorbância medida para o solvente puro foi subtraída da absorbância obtida para a sonda em solução, dessa forma, são eliminadas as contribuições devido ao solvente e à cubeta.

\subsubsection{Diferentes concentrações do soluto}

Uma alíquota foi retirada da solução estoque descrita anteriormente, contendo uma concentração da sonda de $0.1 \mathrm{mM}$. Fizemos a secagem da alíquota para evaporação do solvente à temperatura ambiente sobre pressão reduzida. Em seguida, a concentração da sonda foi diluída em metanol e acetonitrila considerando diferentes 
regiões para o $p H_{a p}$ da solução.

Procedimento em metanol: Preparamos uma solução ácida com $p H_{a p} \sim 0.3$ obtida pela adição de $\mathrm{HCl}$ resultando numa solução de ácido hidroclorídrico com metanol na proporção de 1:500 em v:v, e uma solução alcalina com $p H_{a p} \sim 12.6$ obtida pela adição de $\mathrm{NaOH}$ na concentração de $22 \mathrm{mM}$. Posteriormente, em cada uma das soluções com $p H_{a p}$ distintos, nós variamos a concentração da sonda obtendo valores que cobriam o intervalo entre $0.1 \mathrm{mM}$ e $0.004 \mathrm{mM}$. Nós medimos o pH da solução com o pHmetro e em seguida o espectro UV-vis com o espectrofotômetro.

Procedimento em acetonitrila: Preparamos duas soluções estoque, a primeira com excesso de $\mathrm{HCl}\left(\mathrm{pH}\right.$ ácido, $\mathrm{pH}_{a p}=0.0$ ) e concentração $10 \mathrm{mM}$ de Merocianina de Brooker e a segunda com excesso de $\mathrm{NaOH}\left(\mathrm{pH}\right.$ alcalino, $\mathrm{pH}_{a p}=13.3$ ) e concentração $10 \mathrm{mM}$ de Merocianina de Brooker. Isso foi feito para garantirmos a presença de uma única forma, protonada na solução estoque ácida e neutra na solução estoque alcalina. Essas duas soluções estoque, ácida e alcalina, foram submetidas ao mesmo procedimento de secagem. Posteriormente, utilizando a solução com $\mathrm{pH}_{a p}=13.5$, nós variamos a concentração da sonda. Nós medimos o pH da solução com o pHmetro e em seguida o espectro UV-vis com o espectrofotômetro.

\subsubsection{Procedimento com Ultrassom}

Preparamos uma solução estoque de metanol alcalino com excesso de $\mathrm{NaOH}$ $\left(\mathrm{pH}\right.$ alcalino, $\left.\mathrm{pH}_{a p}=13.3\right)$ e concentração $10 \mathrm{mM}$ de Merocianina de Brooker. Isso foi feito para garantirmos a presença de uma única forma da molécula. Essa solução estoque alcalina, foi submetida ao mesmo procedimento de secagem, descrito anteriormente. Posteriormente preparamos filmes em tubos de ensaio através da evaporação do metanol e depois foi adicionado o solvente acetonitrila. Uma amostra de $0.5 \mathrm{~mL}$ foi colocada no ultrasom de banho (Thornton de 40kHz) por 30 min. Em seguida, a amostra foi colocada no espectofotômetro e as medidas do espectro UV-vis foram 
feitas marcando o intervalo de tempo entre elas. 


\section{Capítulo 5}

\section{Merocianina de Brooker: Resultados teóricos}

Buscando descrever os efeitos de solventes na estrutura eletrônica da sonda MB, nós realizamos o estudo teórico através de cálculos quânticos e simulaçoes computacionais. Nesse estudo analisamos algumas possibilidades estruturais para a sonda, como as formas neutra e protonada. Estudamos o efeito de diferentes solventes no espectro eletrônico de absorção e através de cálculos de energia livre determinamos os valores de $p K_{a}$ associados aos processos de protonação/desprotonação.

\subsection{Estudo da molécula isolada}

\subsubsection{Geometrias}

O primeiro passo na caracterização teórica de uma dada molécula é a obtenção das suas propriedades eletrônicas e estruturais, como exemplo, a sua geometria de mínima energia, momento de dipolo, espectro eletrônico, espectro vibracional, NMR, entre outras. Para a obtenção da geometria da sonda MB nós utilizamos o método DFT com dois funcionais B3LYP e PBE com o conjunto de funções base 6-311+G**. Nós estudamos a molécula na forma neutra nas seguintes estruturas para o estado 
fundamental: trans (trans-MB), cis (cis-MB) e trans-zwiteriônica (z-MB). Estudamos também a molécula na sua forma trans protonada $\left(\mathrm{MBH}^{+}\right)$e o dímero antiparalelo $\left(\mathrm{MB}_{2}\right)$. Essas 5 formas diferentes da Meocianina de Brooker foram estudadas na tentativa de compreender as 3 hipóteses existentes (discutidas no capítulo 1) para interpretar o solvatocromismo anômalo desta sonda. As ilustrações das estruturas que foram otimizada estão apresentadas nas figuras $5.1 \mathrm{e} 5.2$.

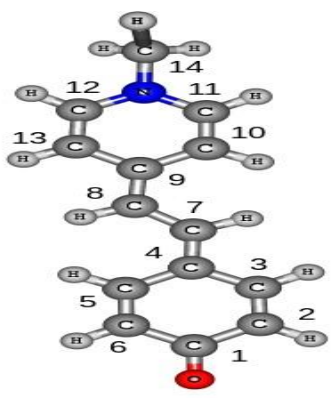

(a) trans-MB ou z-MB

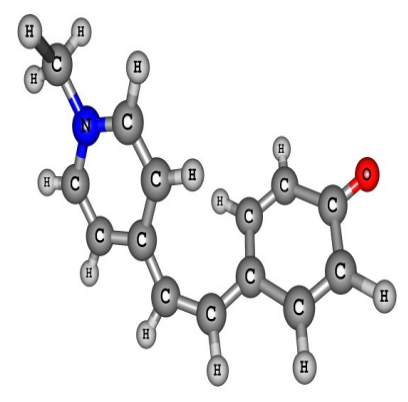

(b) cis-MB

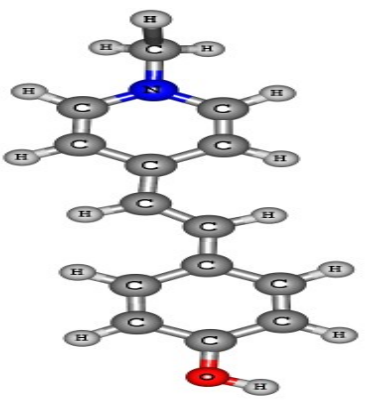

(c) $\mathrm{MBH}^{+}$

Figura 5.1: Ilustração das estruturas da sonda MB que foram otimizadas, indicando a numeração utilizada nas análises seguintes.

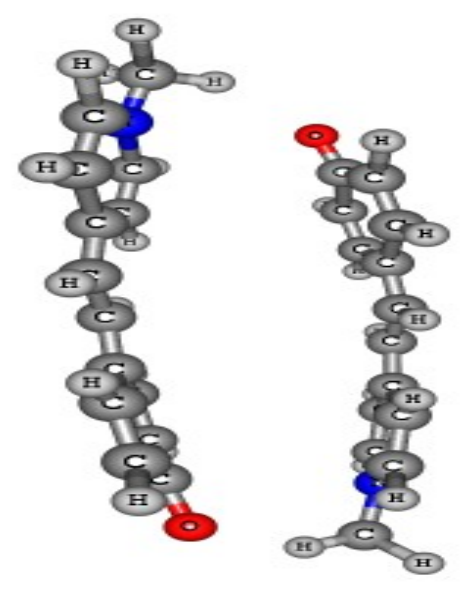

Figura 5.2: Ilustração do dímero antiparalelo da $\mathrm{MB},\left(\mathrm{MB}_{2}\right)$.

A forma trans-MB foi obtida naturalmente otimizando a estrutura do estado fundamental. Essa é a estrutura de menor energia para a molécula isolada. A forma 
cis-MB foi obtida realizando uma rotação na cadeia de átomos $\mathrm{C} 4-\mathrm{C} 7-\mathrm{C} 8-\mathrm{C} 9$, ver numeração na figura 5.1. A forma protonada $\left(\mathrm{MBH}^{+}\right)$foi obtida pela adição de um próton $\mathrm{H}^{+}$junto ao oxigênio da trans-MB e reotimizando a estrutura. A partir da forma protonada, nós obtivemos a forma zwiteriônica (z-MB), retirando o próton da $\mathrm{MBH}^{+}$otimizada e não permitindo a relaxação da geometria. O dímero foi obtido a partir do posicionamento antiparalelo de duas MB na forma trans.

Nós analisamos algumas diferenças geométricas nessas diversas estruturas estudadas. Na tabela 5.1 apresentamos algumas ligações químicas que apresentaram maiores mudanças. Podemos observar que as formas trans e cis-MB apresentam as ligações $\mathrm{C}=\mathrm{O}$ e $\mathrm{C} 2=\mathrm{C} 3$ curtas (com valores típicos de ligação dupla) e ligação $\mathrm{C} 1-\mathrm{C} 2$ longa (com valor típico de ligação simples). Desta forma, confirmamos a informação presente nos artigos teóricos 223,27] da MB que mostram um caráter quinoidal (sem separação de cargas, ver firgura 1.1) para a MB otimizada em vácuo.

A forma protonada $\mathrm{MBH}^{+}$apresenta as ligações C-O e C4-C7 longas, com valores típicos de ligação simples, confirmando um caráter benzoidal. Retirando o $\mathrm{H}^{+}$dessa geometria (sem relaxação), geramos uma forma zwiteriônica com separação de cargas, ver figura 1.1 .

Analisando a diferença de energia entre essas formas da MB, verificamos que a trans-MB é a mais estável por $6.40 \mathrm{kcal} / \mathrm{mol}$ em relação a cis-MB e por $12.28 \mathrm{kcal} / \mathrm{mol}$ em relação a z-MB. O dipolo da z-MB é maior, 17.98 D, comparativamente ao dipolo da trans-MB, 14.48 D. Sendo assim, em solução aquosa ou solventes polares a forma z-MB ou uma forma ressonante intermediária entre a trans-MB e z-MB, pode ser estabilizada pelo meio, devido a interação soluto-solvente (interação dipolo-dipolo sendo mais forte) que pode compensar a variação de energia intramolecular para mudar da forma transMB para z-MB.

Analisando a geometria e distribuição de cargas do dímero antiparalelo, $\mathrm{MB}_{2}$, (ver ligações químicas e dipolo na tabela 5.1) percebemos que as ligações de cada 
Tabela 5.1: Valores para o comprimento da ligação $(\AA)$, momento de dipolo (D), diferenças de energia relativa à conformação trans $\Delta \mathrm{E}(\mathrm{kcal} / \mathrm{mol})$, comprimento de onda de máxima absorção $\left(\lambda_{\max } \mathrm{em} \mathrm{nm}\right)$ e a força do oscilador da excitação eletrônica em parênteses para a sonda MB nas diferentes estruturas. Valores obtidos para a molécula isolada, otimizada em nível B3LYP /6-311+G**. A numeração dos átomos na tabela está de acordo com a descrição da figura 5.1 .

\begin{tabular}{ccccc}
\hline \hline Distância & trans-MB & cis-MB & $\mathrm{MBH}^{+} / \mathrm{z}-\mathrm{MB}$ & $\mathrm{MB}_{2}$ \\
\hline O-C & 1.236 & 1.236 & 1.346 & 1.253 \\
C1-C2 & 1.469 & 1.469 & 1.407 & 1.458 \\
C2-C3 & 1.355 & 1.356 & 1.377 & 1.363 \\
C4-C7 & 1.390 & 1.390 & 1.438 & 1.404 \\
C7-C8 & 1.407 & 1.417 & 1.365 & 1.390 \\
\hline Dipolo $(\mathrm{D})$ & 14.48 & 11.25 & $10.68 / 17.98$ & $0.00 / 16-96$ \\
$\Delta \mathrm{E}(\mathrm{kcal} / \mathrm{mol})$ & 0.00 & 6.40 & $-266.39 / 12.28$ & -15.28 \\
$\lambda_{\text {max }}(\mathrm{OSC})$ & $430(1.52)$ & $460(0.77)$ & $395(1.18) / 480(1.47)$ & $618\left(10^{-4}\right)$ \\
\hline \hline
\end{tabular}


monômero de MB são idênticas e a forma de cada nomômero de MB é intermediária entre a trans-MB (quinoidal/neutra) e a z-MB (benzoidal/zwiteriônica). Pois esse dímero promove a formação de duas ligações de hidrogênio (LH) entre o oxigênio da carbonila e um hidrogênio da metila. As distâncias O ‥H são de 2.208 Ảe os ângulos $\mathrm{O} \cdots \mathrm{CH}$ são de $22.8^{\circ}$. Essas LH além de induzir um estiramento das ligações C-O, ainda provocam uma separação de carga intramolecular em cada monômero da MB, que aumenta o dipolo de cerca de 14.5D (MB isolada) para cerca de 17D (nomômero de MB no dímero). Devido ao grande dipolo de cada monômero do dímero e às duas LH entre os monômeros, existe uma forte estabilização do dímero antiparalelo da MB provocando uma energia de ligação de $15.3 \mathrm{kcal} / \mathrm{mol}\left(E_{l i g}=E\left(M B_{2}\right)-2 E(M B)\right.$ incluindo correção de counterpoise). Adicionalmente, o dipolo do dímero é nulo e isso favorece sua solvatação em solventes apolares.

\subsubsection{Espectro de Absorção}

Na tabela 5.1 também listamos os valores do comprimento de onda $\left(\lambda_{\max }\right)$ e da força do oscilador (OSC) da primeira excitação eletrônica calculados com o método TD-B3LYP $/ 6-311+G^{* *}$. Note que as formas trans-, cis- e z-MB em vácuo têm transição muito próximas: $430 \mathrm{~nm}\left(23255 \mathrm{~cm}^{-1}\right), 460 \mathrm{~nm}\left(21740 \mathrm{~cm}^{-1}\right)$ e $480 \mathrm{~nm}(20830$ $\left.\mathrm{cm}^{-1}\right)$, respectivamente. Porém as transições da trans- e z-MB é cerca de duas vezes mais intensa que a cis-MB, pois a intensidade da excitação é proporcional a OSC. Já o dímero $\mathrm{MB}_{2}$ em vácuo tem uma transição calculada em $618 \mathrm{~nm}\left(16180 \mathrm{~cm}^{-1}\right)$, porém com intensidade muito baixa comparativamente ao monômero. Outras duas transições são calculadas para o dímero em vácuo em 615 e 510 nm também com baixa intensidade $\left(\mathrm{OSC} \approx 10^{-4}\right)$. Com intensidade alta (OSC $\left.=1.63\right)$, foi calculada uma transição em $418 \mathrm{~nm}\left(23920 \mathrm{~cm}^{-1}\right)$. As transições eletrônicas apresentadas na tabela 5.1 são referentes a excitações que envolvem os dois orbitais mais altos ocupados (HOMO e HOMO-1) e os dois orbitais mais baixos desocupados, ou virtuais (LUMO e 
LUMO+1). No caso do dímero a excitação envolve os quatro orbitais mais altos ocupados (HOMO até HOMO-3) e os quatro orbitais mais baixos desocupados (LUMO até $\mathrm{LUMO}+3)$. Todos esses orbitais são delocalizados sobre quase toda a molécula e perpendiculares às ligações químicas. Portanto as excitações são caracterizadas como $\pi \rightarrow \pi^{*}$. Entretanto os orbitais ocupados envolvidos nas excitações apresentam maior densidade eletrônica próximo a ligação C-O e os orbitais virtuais envolvidos próximo a ligação N-CH3. Assim, observamos um caráter de transferência de carga intramolecular (ICT) na excitação eletrônica. Na figura 5.3 apresentamos a ilustração dos orbitais envolvidos na transição eletrônica.

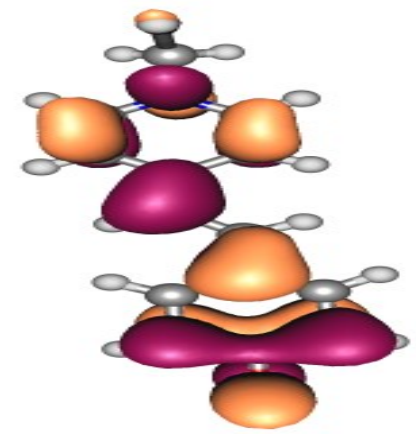

(a) $\operatorname{HOMO}(\pi)$

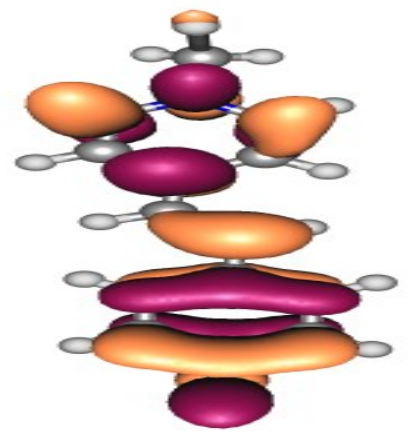

(b) $\operatorname{LUMO}\left(\pi^{*}\right)$

Figura 5.3: Ilustração dos orbitais moleculares para as sonda MB obtidos com TD-B3LYP/6$311+\mathrm{G}^{* *}$.

Do ponto de vista experimental (como descrito no capítulo 1), existem vários artigos na literatura que reportam medidas do espectro eletrônico de absorção da MB com máximo de absorção entre $620 \mathrm{~nm}$ em solução de clorofórmio a $440 \mathrm{~nm}$ em solução aquosa alcalina $22,25,32-37]$. Sendo assim, é interessante observar que as três formas estudadas da MB (trans, cis e zwiteriônica) apresentam comprimento de onda próximo da medida em água (solvente de maior polaridade) e não do clorofórmio (solvente de menor polaridade). Usualmente, é esperado que os valores calculados para moléculas em vácuo sejam próximos dos valores medidos para solventes de baixa polaridade (em nosso caso clorofórmio) e não para o solvente de máxima polaridade 
(água). Isso nos fez questionar a confiabilidade do método utilizado para o cálculo da transição eletrônica (TD-B3LYP). Em seguida, retornaremos a este ponto e analisaremos vários métodos para o cálculo dessas transições da $\mathrm{MB}$ em vácuo. Por outro lado, é importante ressaltar dois outros aspectos que podemos analisar com base nos $\lambda_{\max }$ calculados: (i) o valor do dímero está próximo ao valor medido em clorofórmio (solvente apolar), onde a MB não apresenta boa solubilidade; e (ii) o valor da MB protonada, $\lambda_{\max }\left(\mathrm{MBH}^{+}\right)=395 \mathrm{~nm}\left(25315 \mathrm{~cm}^{-1}\right)$, está muito próximo do valor medido entre $397 \mathrm{~nm}$ em solução de clorofórmio a $374 \mathrm{~nm}$ em solução aquosa alcalina [dados medidos neste trabalho que serão apresentados no capítulo 7]. Essa concordância entre os valores calculados e os medidos indicam uma confiabilidade do método utilizado para o cálculo, TD-B3LYP. Na literatura, é senso comum que inúmeros funcionais de densidade apresentam valores concordantes para excitação eletrônica dentro de uma variação de $20 \mathrm{~nm}$. Na tabela 5.2, mostramos os resultados obtidos para diferentes funcionais e para o método multiconfiguracional EOM-CCSD (Equation Of Motion-Coupled Cluster Singles and Doubles), considerado um bom método para cálculo de excitações eletrônicas. Pelo fato da molécula ser muito grande, só conseguimos otimizá-la com DFT híbrido (B3LYP) e puro (PBE) na forma trans para realizar esse estudo comparativo de métodos teóricos.

Tabela 5.2: Valores da primeira energia de transição eletrônica $\left(\mathrm{em} \mathrm{cm}^{-1}\right)$, comprimento de onda (em nm) e força do oscilador (OSC) calculados para sonda trans-MB em vácuo com diferentes métodos de mecânica quântica com o conjunto de funções base $6-311+\mathrm{G}^{* *}$.

\begin{tabular}{cccc}
\hline \hline Método de cálculo & $\mathrm{E}\left(\mathrm{cm}^{-1}\right)$ & $\lambda(\mathrm{nm})$ & OSC \\
\hline Geometria B3LYP $/ 6-311+\mathrm{G}^{* *}$ & & & \\
\hline B3LYP & 22643 & 442 & 0.8887
\end{tabular}




\begin{tabular}{cccc} 
O3LYP & 18315 & 546 & 0.0000 \\
& 21930 & 456 & 0.2633 \\
X3LYP & 21459 & 466 & 0.0000 \\
& 22727 & 440 & 1.0191 \\
BHandH & 23866 & 419 & 1.5649 \\
BHandHLYP & 23923 & 418 & 1.5876 \\
CAM-B3LYP & 23288 & 429 & 1.5160 \\
WB97XD & 23256 & 430 & 1.5270 \\
\hline
\end{tabular}


Tabela 5.2 - Continuação da página anterior

\begin{tabular}{cccc} 
LC-WPBE & 23697 & 422 & 1.6165 \\
LC-BLYP & 23866 & 419 & 1.6449 \\
HCTH407 & 14556 & 687 & 0.0000 \\
M06L & 20000 & 500 & 0.0240 \\
& 16667 & 600 & 0.0000 \\
Geometria PBE/6-311+G** & 21552 & 464 & 0.0461 \\
\hline B3LYP & & & \\
BHandHLYP & 22072 & 453 & 0.7740 \\
CAM-B3LYP & 23301 & 429 & 1.5911 \\
WB97XD & 22696 & 441 & 1.5210 \\
LC-WPBE & 22646 & 442 & 1.5290 \\
M06L & 22978 & 435 & 1.6179 \\
\hline \hline & 13947 & 717 & 0.0000 \\
\hline EOM-CCSD/6-31G* & 24197 & 413 & \\
\hline \hline
\end{tabular}

Note que na geometria PBE, os valores da energia da transição eletrônica de todos os funcionais variam entre 22072 a $23301 \mathrm{~cm}^{-1}$, com exceção do M06L $\left(13947 \mathrm{~cm}^{-1}\right)$. Esses valores estão razoáveis comparativamente ao do EOM-CCSD $\left(24197 \mathrm{~cm}^{-1}\right)$. Esse último cálculo foi feito com uma base menor, 6-31G*, devido ao grande tamanho da molécula. Fizemos testes anteriores, e aumentando o tamanho do conjunto de funções base a energia da transição tende a diminuir até atingir uma convergência. Sendo 
assim, para a base $6-311+\mathrm{G}^{*}$, esperamos obter valores com EOM, mais próximos dos obtidos com os DFT. Os valores para as duas geometrias e mesmos funcionais diferem por cerca de $600 \mathrm{~cm}^{-1}$, com exceção novamente para o M06L. Alguns funcionais apresentam a primeira transição proibida, com OSC nulo. Nestes casos apresentamos também o valor da segunda transição. Para esses funcionais identificamos que essas transições proibidas têm energia bem menores que as demais, portanto mais próximos dos valores experimentais. Porém elas apresentam caráter $n \rightarrow \pi^{*}$ e não $\pi \rightarrow \pi^{*}$ como caracterizado experimentalmente. Sendo assim, esses funcionais foram descartados. Os demais funcionais apresentam valores muito próximos, daí optamos pelos funcionais B3LYP (que é tradicional na literatura) e CAM-B3LYP por apresentar correção de longo alcance no termo Coulombiano e por isso ser também amplamente utilizado para cálculo de excitações que apresentam caráter ICT. Os demais cálculos de excitação eletrônica, apresentados neste trabalho, serão realizados com apenas os métodos TDB3LYP e TD-CAM-B3LYP e funções base 6-311+G**.

\subsubsection{Efeito Solvente: modelo contínuo}

É importante incluir os efeitos de solventes para compreendermos o papel do solvente em cada forma estudada. Nesta etapa, não utilizamos a forma cis-MB, devido ao fato dela ter: (i) energia intramolecular maior que a trans-MB, (ii) menor dipolo, (iii) transição eletrônica na mesma região que a trans-MB, e (iv) intensidade de absorção bem menor que a trans-MB. Portanto, caso a forma cis-MB coexista com a forma trans-MB em um solvente específico, ela terá menor população (devido a diferença de energia) e menor influência no espectro de absorção eletrônica (devido a menor absorbância). Sendo assim, de agora em diante desprezaremos seu efeito e ficaremos com apenas duas hipóteses para o solvatocromismo anômalo da MB, a devido a mudança de forma neutra/zwiteriônica e a devido a agregação. Os efeitos do solvente serão considerados inicialmente utilizando o modelo contínuo polarizável PCM e 
em seguida um modelo discreto onde as moléculas do solvente serão consideradas explicitamente no cálculo quântico do espectro, a partir de configurações soluto-solvente geradas em simulações com método Monte Carlo com campo de força clássico. Os resultados obtidos nestas etapas de inclusão dos efeitos de solvente com modelo contínuo serão apresentados e discutidos a seguir.

\section{Geometria}

As formas trans-MB e $\mathrm{MBH}^{+}$foram otimizadas com B3LYP $/ 6-311+\mathrm{G}^{* *}$, a partir das geometrias previamente obtidas em vácuo. Na tabela 5.3 apresentamos as mesmas ligações químicas apresentadas na tabela 5.1 para as geometrias otimizadas em vácuo. Verificamos que o modelo contínuo não é sensível a variação da constante dielétrica entre 78.36 para água a 24.85 para etanol, pois as mudanças de geometria são desprezíveis. Comparativamente a geometria de vácuo existe um pequeno estiramento ( $0.02 \AA$ ) nas ligações C-O e C4-C7, e um pequeno encurtamento de mesma magnitude nas ligações C2-C3 e C7-C8, aumentando ligeiramente o caráter benzoidal/zwiteriônico das formas $\mathrm{MB}$ e $\mathrm{MBH}^{+}$. Comparando as geometrias das duas formas obtidas com PCM para água e com a experimental [103] obtida para um cristal tri-hidratado, verificamos que a experimental é uma forma benzoidal intermediária entre a $\mathrm{MB}$ e $\mathrm{MBH}^{+}$. 
Tabela 5.3: Valores para o comprimento da ligação $(\AA)$ para a sonda MB em diferentes solventes. Entre parênteses apresentamos as informações para a molécula protonada. A geometria foi otimizada em nível B3LYP/6-311+G** e o solvente considerado através do modelo contínuo polarizável PCM. A numeração dos átomos na tabela segue a descrição da figura 5.1 .

\begin{tabular}{|c|c|c|c|c|c|}
\hline Solvente & $\mathrm{O}-\mathrm{C}$ & $\mathrm{C} 1-\mathrm{C} 2$ & C2-C3 & C4-C7 & $\mathrm{C} 7-\mathrm{C} 8$ \\
\hline \multirow[t]{2}{*}{ Água $\left(E_{T}(30)=63.1\right)$} & 1.265 & 1.451 & 1.369 & 1.418 & 1.379 \\
\hline & $(1.359)$ & $(1.402)$ & $(1.382)$ & $(1.451)$ & $(1.355)$ \\
\hline \multirow[t]{2}{*}{ Metanol $\left(E_{T}(30)=55.4\right)$} & 1.264 & 1.451 & 1.368 & 1.417 & 1.380 \\
\hline & $(1.359)$ & $(1.402)$ & $(1.382)$ & $(1.451)$ & $(1.355)$ \\
\hline \multirow[t]{2}{*}{ Etanol $\left(E_{T}(30)=51.9\right)$} & 1.263 & 1.452 & 1.368 & 1.416 & 1.381 \\
\hline & $(1.359)$ & $(1.403)$ & $(1.382)$ & $(1.451)$ & $(1.356)$ \\
\hline \multirow[t]{2}{*}{ Acetonitrila $\left(E_{T}(30)=45.6\right)$} & 1.264 & 1.451 & 1.368 & 1.417 & 1.380 \\
\hline & $(1.359)$ & $(1.402)$ & $(1.382)$ & $(1.451)$ & $(1.355)$ \\
\hline \multirow[t]{2}{*}{$\operatorname{DMSO}\left(E_{T}(30)=45.1\right)$} & 1.264 & 1.451 & 1.369 & 1.418 & 1.380 \\
\hline & $(1.359)$ & $(1.402)$ & $(1.382)$ & $(1.451)$ & $(1.355)$ \\
\hline \multirow[t]{2}{*}{ Clorofórmio $\left(E_{T}(30)=39.1\right)$} & 1.254 & 1.457 & 1.363 & 1.406 & 1.390 \\
\hline & $(1.356)$ & $(1.404)$ & $(1.381)$ & $(1.448)$ & $(1.358)$ \\
\hline \multirow[t]{2}{*}{ Isolada } & 1.236 & 1.469 & 1.355 & 1.390 & 1.407 \\
\hline & $(1.346)$ & $(1.407)$ & $(1.377)$ & $(1.438)$ & $(1.356)$ \\
\hline Cristal tri-hidratado $|103|$ & 1.304 & 1.414 & 1.379 & 1.441 & 1.346 \\
\hline
\end{tabular}


Na tabela 5.4 mostramos os momentos de dipolo e as cargas atômicas obtidas do ajuste com o método CHELPG do potencial eletrostático calculado com MP2/6$311+\mathrm{G}^{* *} / \mathrm{PCM}$ a partir das geometrias obtidas com os diferentes solventes. Verificamos novamente que o modelo contínuo não é sensível a variação da constante dielétrica entre água e etanol, pois as mudanças das cargas atômicas e dipolo são pequenas. Porém, observamos um grande efeito de polarização da trans-MB. Fazendo com seu dipolo em vácuo aumente de $14.5 \mathrm{D}$ para 31 a 32D em solvente. O átomo que apresenta uma maior variação na carga é o oxigênio (de cerca de -0.60 e em vácuo para -0.83 em solução). Essa carga não vem majoritariamente do nitrogênio, como esperado, mas sim de toda a molécula.

Tabela 5.4: Valores do momento de dipolo (D) e carga atômica (e) para a sonda MB em diferentes solventes. Geometria otimizada em nível B3LYP/6-311 $+\mathrm{G}^{* *}$ e momento de dipolo obtido com MP2/6-311 $+\mathrm{G}^{* *}$. A numeração dos átomos na tabela segue a descrição da figura 1.1

\begin{tabular}{ccccccc}
\hline \hline Solvente & Carga(N) & Carga(C7) & Carga(C8) & Carga(C1) & Carga(O) & Dipolo (D) \\
\hline Água & 0.122 & -0.340 & -0.114 & 0.646 & -0.836 & 31.94 \\
Metanol & 0.124 & -0.340 & -0.111 & 0.654 & -0.828 & 31.18 \\
Etanol & 0.128 & -0.338 & -0.113 & 0.654 & -0.822 & 30.78 \\
Acetonitrila & 0.125 & -0.341 & -0.111 & 0.654 & -0.829 & 31.29 \\
DMSO & 0.123 & -0.334 & -0.118 & 0.651 & -0.832 & 31.57 \\
Clorofórmio & 0.132 & -0.349 & -0.115 & 0.658 & -0.742 & 24.71 \\
Isolado & 0.100 & -0.355 & -0.109 & 0.662 & -0.599 & 14.48 \\
\hline \hline
\end{tabular}




\section{Espectro de absorção}

Nas tabelas 5.5 e 5.9 apresentamos os resultados obtidos para a primeira transição eletrônica da sonda MB nos mesmos solventes da tabela anterior. Consideramos as formas neutra (trans-MB) e protonadas $\left(\mathrm{MBH}^{+}\right)$, as geometrias do estado fundamental foram otimizadas nos respectivos solventes usando o modelo contínuo PCM e discutidas nas tabelas 5.3 e 5.4

Analisando os valores calculados em clorofórmio, observamos valores em cerca de $21000 \mathrm{~cm}^{-1}$. Embora a inclusão do solvente tenha deslocado a transição para menor energia, os valores ainda estão muito discrepantes em relação ao resultado experimental, com uma diferença de cerca de $5000 \mathrm{~cm}^{-1}$. Já as transições eletrônicas calculadas em água estão em cerca de $21400 \mathrm{~cm}^{-1}$. Esses valores estão deslocados apenas cerca

de $1000 \mathrm{~cm}^{-1}$ do valor experimental de aproximadamente $22500 \mathrm{~cm}^{-1}$. Isso mostra uma boa concordância do valor teórico e experimental, considerando que o PCM é um modelo simples e que uma melhor descrição do solvente pode facilmente melhorar esse resultado. Sendo assim, com base nesses resultados verificamos que existe algum problema na descrição da MB em vácuo e em solventes de baixa polaridade. 
Tabela 5.5: Valores para energia de transição $\left(\mathrm{em} \mathrm{cm}^{-1}\right)$, comprimento de onda da excitação $\mathrm{nm}$ e força do oscilador da transição (OSC) obtidos para diferentes solventes e com dois funcionais distintos: CAM-B3LYP e B3LYP e o conjunto de funções base 6-311+G**. Forma $\mathrm{MBH}^{+}$.

\begin{tabular}{ccccc}
\hline \hline & \multicolumn{2}{c}{ CAM-B3LYP } & \multicolumn{2}{c}{ B3LYP } \\
\hline MBH & & MBH $^{+}$ & \\
\hline Solvente & E(OSC) & $\lambda(n m)$ & E(OSC) & $\lambda(n m)$ \\
Água & $27322(1.21)$ & 366 & $24510(1.10)$ & 408 \\
Metanol & $26316(1.26)$ & 380 & $24096(1.12)$ & 415 \\
Etanol & $27027(1.22)$ & 370 & $24331(1.11)$ & 411 \\
Acetonitrila & $27174(1.21)$ & 368 & $24450(1.10)$ & 409 \\
DMSO & $27027(1.23)$ & 370 & $24272(1.12)$ & 412 \\
Clorofórmio & $25974(1.25)$ & 385 & $23474(1.14)$ & 426 \\
\hline \hline
\end{tabular}


Tabela 5.6: Valores para energia de transição $\left(\mathrm{em} \mathrm{cm}^{-1}\right)$, comprimento de onda da excitação $\mathrm{nm}$ e força do oscilador da transição (OSC) obtidos para diferentes solventes e com dois funcionais distintos: CAM-B3LYP e B3LYP e o conjunto de funções base $6-311+\mathrm{G}^{* *}$. Forma MB.

\begin{tabular}{ccccc}
\hline \hline & \multicolumn{2}{c}{ CAM-B3LYP } & B3LYP \\
\hline \multicolumn{3}{c}{ MB } & MB \\
\hline Solvente & $\mathrm{E}(\mathrm{OSC})$ & $\lambda(n m)$ & $\mathrm{E}(\mathrm{OSC})$ & $\lambda(\mathrm{nm})$ \\
Água & $21552(1.51)$ & 464 & $20921(1.43)$ & 478 \\
Metanol & $21459(1.53)$ & 466 & $20921(1.44)$ & 478 \\
Etanol & $21322(1.54)$ & 469 & $20790(1.45)$ & 481 \\
Acetonitrila & $21459(1.53)$ & 466 & $20833(1.44)$ & 480 \\
DMSO & $21231(1.54)$ & 471 & $20619(1.46)$ & 485 \\
Clorofórmio & $20619(1.64)$ & 485 & $20492(1.55)$ & 488 \\
\hline \hline
\end{tabular}

Observa-se nas tabelas 5.5 e 5.6 que o efeito do solvente contínuo na excitação eletrônica das formas $\mathrm{MB}$ e $\mathrm{MBH}^{+}$provoca pequenos deslocamentos no comprimento de onda da excitação. Comparando os resultados das tabelas 5.5 e 5.6 com os nossos valores experimentais obtidos para diferentes solventes apresentados na tabela 5.7] [os resultados experimentais serão discutidos de maneira mais aprofundada no capítulo 7.], observa-se que o modelo contínuo não consegue descreve o forte solvatocromismo apresentado pela MB. Por exemplo, observamos experimentalmente um deslocamento de aproximadamente $170 \mathrm{~nm}$ na mudança água-clorofórmio e o modelo teórico descreve apenas um deslocamento de $10 \mathrm{~nm}$. 
Tabela 5.7: Valores experimentais para o comprimento de onda de máxima absorção, $\lambda_{\max }$, das formas $\mathrm{MB}$ e $\mathrm{MBH}^{+}$. O erro experimental é de $1 \mathrm{~nm}$.

\begin{tabular}{ccc}
\hline \hline Solvente & $\lambda_{1}(n m)$ & $\lambda_{2}(n m)$ \\
\hline Água & protonada $\mathrm{MBH}^{+}$ & desprotonada $M B$ \\
Metanol & 371 & 444 \\
Etanol & 400 & 485 \\
Acetonitrila & 382 & 513 \\
DMSO & 391 & 567 \\
Clorofórmio & 397 & 573 \\
\hline \hline
\end{tabular}

Observa-se também que a transição eletrônica para a forma protonada está relativamente bem descrita pelos funcional utilizados, onde os valores obtidos pelo funcional CAM-B3LYP está deslocado para valores menores comprimentos de onda, comparado aos valores experimentais, e o funcional B3LYP desloca a transição para valores maiores de comprimentos de onda. Nesse ponto vale ressaltar que o modelo para o solvente utilizado ainda é limitado, acreditamos que com uma descrição mais realística para o solvente através de simulações clássicas, onde o solvente é tratado demaneira discreta, nós teremos uma melhora nos nosso valores teóricos. Devemos mencionar também que uma das fontes de erro no nosso deslocamento teórico advém das dificuldades apresentadas anteriormente para a descrição correta da transição eletrônica em solventes de baixa polaridade. 


\subsubsection{Efeito Solvente: modelo discreto}

Uma etapa natural na evolução da descrição do solvente, é a inclusão do solvente através do modelo discreto. Dessa forma, dando prosseguimento ao nosso estudo, nós realizamos cálculos para a sonda solvatada. Foram realizadas simulações clássicas nos seguintes solventes: água, metanol e acetonitrila. As configurações das sondas solvatadas nos diferentes solventes foram geradas através de simulações computacionais de líquidos moleculares, usando o método Monte Carlo Metropolis. O nosso estudo foi feito considerando as moléculas rígidas. A geometria da sonda MB foi obtida no respectivo solvente usando o modelo contínuo PCM com o método B3LYP/6-311+G** e o conjunto de cargas atômicas para o soluto foi obtido através do ajuste do potencial eletrostático obtido com o método MP2/6-311 $+\mathrm{G}^{* *}$ também na presença do solvente. O ensemble utilizado nas simulações foi o NPT, em que são mantidos fixos o número de partículas $(\mathrm{N})$, a pressão $(\mathrm{P})$ e a temperatura $(\mathrm{T})$. Para descrever a interação intermolecular entre o soluto e o solvente usamos o potencial Lennard-Jones 101 adicionado à um termo Coulombiano, o potencial é dado pela equação 3.34 . Como a sonda estudada nesse trabalho não está parametrizada para o campo de força, nós obtivemos os parâmetros $\epsilon$ e $\sigma$ para o potencial Lennard-Jones por semelhança com grupos funcionais que possuem parametrização. Esse procedimento foi feito usando o campo de força OPLS-AA [104] para as duas estruturas, neutra e protonada.

Após as simulações, nós realizamos o estudo do efeito do solvente no espectro de absorção das sondas com o modelo discreto para os solventes. Este estudo foi feito utilizando o procedimento sequencial $\mathrm{QM} / \mathrm{MM}$, em que primeiro realizamos as simulações com mecânica molecular para gerar as configurações acessíveis ao sistema e posteriormente cálculos com mecânica quântica são realizados sobre essas configurações para a obtenção da propriedade eletrônica de interesse. Nesse procedimento é feita uma análise estatística sobre as configurações com a intenção de reduzir o número de configurações que serão levadas ao cálculo quântico. Nas nossas simulações 
foram geradas 120 mil configurações sucessivas, destas nós realizamos cálculos quânticos em apenas 120 configurações que estão estatísticamente descorrelacionadas. A seguir apresentamos os nossos resultados obtidos para cada solvente estudado.

\section{Água}

O primeiro solvente que será tratado é a água, um solvente que tem constante dielétrica igual a 78.36. O modelo usado para a água foi o SPC [105]. A simulação foi realizada com o número de partículas igual a 1 molécula do soluto (sonda) mais 1000 moléculas do solvente, a densidade inicial considerada para a água foi $1 \mathrm{~g} / \mathrm{cm}^{3}$, à pressão de 1 atm e temperatura de $298 \mathrm{~K}$. Foram realizados 60 mil ciclos MC na termalização e 120 mil ciclos no equilíbrio. As simulações foram realizadas utilizando condições periódicas de contorno numa caixa cúbica de lado aproximadamente igual a $31.27 \AA$.

O primeiro passo feito na simulação foi a termalização do sistema (sonda + solvente) para garantirmos que esse esteja em equilíbrio termodinâmico. No processo de termalização foram realizados $6 \times 10^{7}$ passos $\mathrm{MC}$, o que equivale à 60 mil ciclos MC, onde um ciclo se completa quando tentamos mover todas as moléculas da caixa de simulação. Na figura 5.4 apresentamos o comportamento da energia por molécula versus o número de ciclos para a sonda MB solvatada em água durante o processo de termalização e equilíbrio do sistema. Vê-se na figura 5.4 que a energia por molécula atinge gradativamente o equilíbrio durante o estágio de termalização e depois flutua em torno de um valor médio durante a etapa de equilíbrio. 


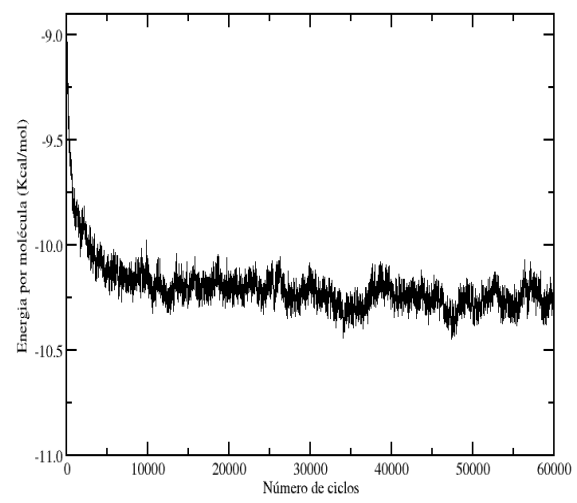

(a) Termalização

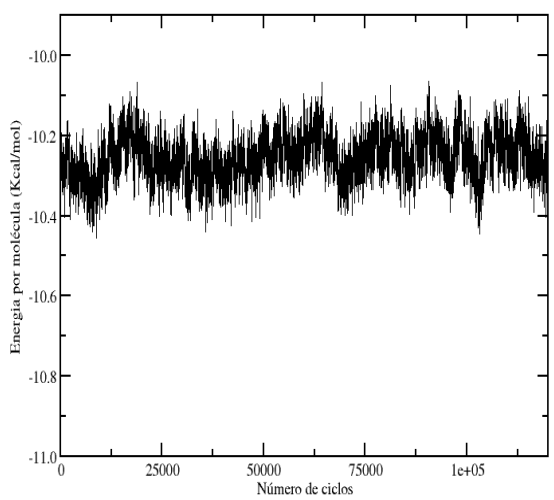

(b) Equilíbrio

Figura 5.4: Energia por molécula versus o número de ciclos MC durante o processo de termalização (a) e durante o equilíbrio (b). Estrutura neutra.

Uma etapa seguinte na análise da simulação clássica é o estudo da distribuição do solvente em torno do soluto, com esse estudo podemos saber se existem moléculas do solvente disponíveis para formar ligações de hidrogênio (LH) com o soluto e também podemos determinar a quantidade de moléculas do solvente em cada camada de solvatação. A análise dessas quantidades são feitas através da função de distribuição radial de pares (RDF). Existem várias possibilidades para o cálculo da RDF, como a RDF entre o centro de massa do soluto e o centro de massa do solvente e a RDF calculada na mínima distância entre os átomos do soluto e os átomos do solvente, MDDF. Essas duas possibilidades citadas são mais usadas para a obtenção das camadas de solvatação. Na figura 5.5 analisamos a função radial de pares entre o oxigênio da sonda e o oxigênio do solvente. A análise da RDF entre o oxigênio do soluto e o oxigênio do solvente, $\mathrm{G}_{O O}(\mathrm{r})$, é importante para obtenção da existência de ligações de hidrogênio. O sistema simulado aqui, MB em água, possui a possibilidade de formação de LH, pois tanto a sonda pode ser aceitadora (forma neutra) ou doadora e aceitadora (forma protonada) de próton para a ligação como também a água que é um solvente prótico. 
Isso é refletido na função $\mathrm{G}_{O O}(\mathrm{r})$, figura 5.5, onde a distância mais provável entre o oxigênio do soluto e o oxigênio do solvente é de aproximadamente $3.0 \AA$, valor obtido para as duas estruturas, neutra e protonada.

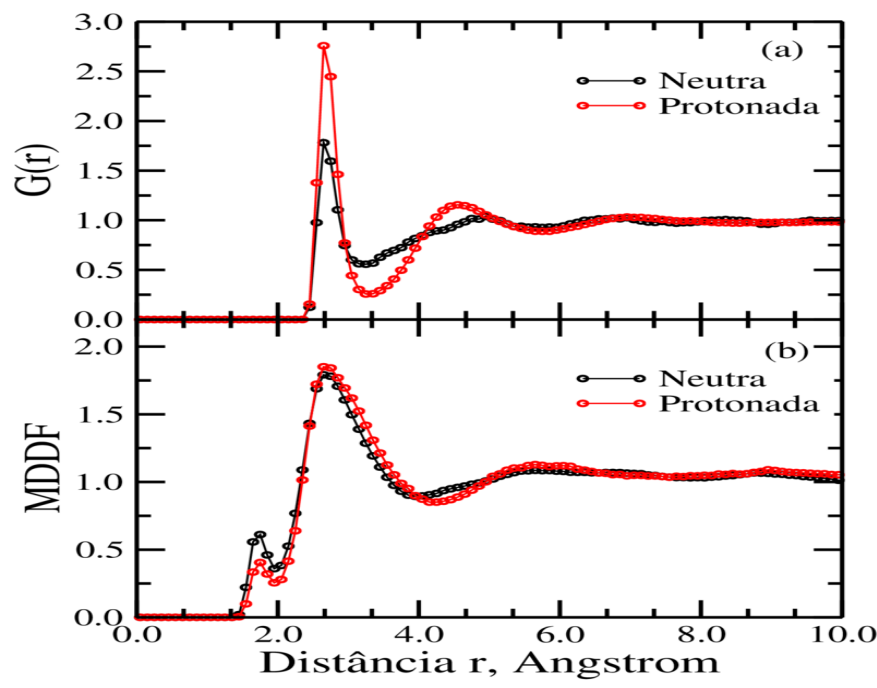

Figura 5.5: Função de distribuição radial de pares, soluto-água. G(r) (a) e MDDF (b).

A obtenção da distribuição do solvente em torno do soluto define as camadas de solvatação. Essa informação com relação ao número de moléculas do solvente que estão mais próximas do soluto será importante posteriormente pois nos permite escolher o tamanho do sistema que será tratado no cálculo quântico para o cálculo do espectro da sonda em solução. Aqui, a informação das camadas de solvatação foi obtida analisando a RDF de mínima distância. Na figura 5.5 apresentamos a MDDF para sonda embebida em água. É possível observar três picos bem definidos para as sondas, um primeiro pico com fim em $2.0 \AA$, o segundo com fim em $3.8 \AA$ e um terceiro com fim em $7.6 \AA$. A integração dessa função em cada um dos picos nos fornece o número de moléculas do solvente existente em cada camada de solvatação, na tabela 5.8 fazemos um sumário com todos os resultados obtidos para as duas estruturas. Observa-se na tabela 5.8 que a distribuição do solvente em torno da sonda na forma neutra e na forma protonada é similar. O limite da caixa possui 500 moléculas de solvente. 
Tabela 5.8: Número de moléculas de água distribuidos ao redor da sonda, obtidos pela integração da função de mínima distância, MDDF.

\begin{tabular}{lccc}
\hline \hline Sonda & Microcamada & $1^{\circ}$ camada & $2^{\circ}$ camada \\
\hline Distância $(\AA)$ & 2.0 & 3.8 & 7.6 \\
MB & 3 & 45 & 194 \\
$\mathrm{MBH}^{+}$ & 2 & 45 & 195 \\
\hline \hline
\end{tabular}

Geradas as configurações pela simulação clássica nós realizamos os cálculos de espectro de absorção sobre as configurações estatisticamente descorrelacionadas obtidas da simulação considerando diferentes formas de incluir o solvente nos cálculos quânticos. Obtivemos as transições eletrônicas para as duas estruturas, neutra MB e protonada $\mathrm{MBH}^{+}$. Os resultados obtidos estão apresentados na tabela 5.9. Na legenda da tabela 5.9, o modelo do solvente ASEC corresponde a uma configuração eletrostática média do solvente [106], que é gerada superpondo todas as configurações estatisticamente descorrelacionadas e dividindo as cargas pelo número de configurações utilizadas. Neste modelo o solvente é considerado apenas como um campo eletrostático médio e apenas um cálculo quântico é realizado para determinar as energias de transição eletrônica do soluto rodeado por cargas pontuais. Dentre os modelos discretos apresentados na tabela 5.9 . esse é o computacionalmente mais rápido. O resultado obtido com ASEC é igual ao obtido quando fazemos a média sobre os resultados de $n$ configurações com a mesma quantidade de moléculas do solvente. O modelo LH significa que foram incluídas explicitamente no cálculo quântico apenas as moléculas do solvente que formam ligação de hidrogênio com o soluto. Neste modelo cada transição eletrônica é calculada como a média sobre 120 cálculos quânticos que incluem uma molécula do soluto com cerca de 2 moléculas do solvente. O modelo LH+500 CP, 
significa que além das moléculas que fazem ligação de hidrogênio, incluimos também o potencial eletrostático produzido pelas demais moléculas do solvente num total de 500 moléculas representadas por cargas pontuais. Neste modelo também foram realizados 120 cálculos quânticos que incluem uma molécula do soluto com cerca de 2 moléculas do solvente rodeados por 1500 cargas pontuais (dos átomos de 500 moléculas de água). O modelo $1 \mathrm{MB}+5 \mathrm{Sol}+495 \mathrm{CP}$, significa que além do soluto, foram incluídas no cálculo quântico 5 moléculas explícitas do solvente mais o potencial eletrostático produzido por 495 moléculas do solvente descritas como cargas pontuais. Estas moléculas foram escolhidas pelo critério de proximidade do soluto, ou seja menor distância solutosolvente. Para este modelo também foram realizados 120 cálculos quânticos. Sendo assim, esse é o modelo discreto de solvatação computacionalmente mais oneroso tanto do ponto de vista de memória como o de tempo de processamento. 
Tabela 5.9: Valores para o comprimento de onda (em $\mathrm{nm})$ da primeira trasnsição eletrônica obtidos para diferentes modelos de solvatação em água. O cálculo foi realizado com o método TD-DFT, funcionais CAM-B3LYP e B3LYP com o conjunto de funções base 6-311+G**. Em parênteses está apresentado o valor para a força do oscilador da respectiva transição (OSC).

\begin{tabular}{ccccc}
\hline \hline Nível de QM & CAM-B3LYP & B3LYP & CAM-B3LYP & B3LYP \\
\hline $\mathrm{X}=$ & $\mathrm{MBH}^{+}$ & $\mathrm{MBH}^{+}$ & $\mathrm{MB}$ & $\mathrm{MB}$ \\
\hline Solvatação & $\lambda(\mathrm{OSC})$ & $\lambda(\mathrm{OSC})$ & $\lambda(\mathrm{OSC})$ & $\lambda($ OSC $)$ \\
$\mathrm{X}+\mathrm{PCM}$ & $366(1.21)$ & $408(1.10)$ & $464(1.51)$ & $478(1.43)$ \\
$\mathrm{X}+\mathrm{ASEC}$ & $360(1.14)$ & $389(1.05)$ & $429(1.42)$ & $431(1.36)$ \\
$\mathrm{X}+\mathrm{LH}$ & $405 \pm 3(1.25)$ & $434 \pm 3(1.08)$ & $434 \pm 2(1.55)$ & $444 \pm 3(0.12)$ \\
$\mathrm{X}+\mathrm{LH}+500 \mathrm{CP}$ & $363 \pm 1(1.17)$ & $393 \pm 1(1.08)$ & $431 \pm 3(1.46)$ & $433 \pm 2(1.39)$ \\
$\mathrm{X}+5$ Sol $+495 \mathrm{CP}$ & $363 \pm 1(1.18)$ & $395 \pm 1(1.09)$ & $434 \pm 4(1.48)$ & $435 \pm 3(1.44)$ \\
\hline & Solução ácida & & Solução alcalina & \\
\hline Experimental em água & $371 \pm 1(0.28)$ & & $444 \pm 1(0.50)$ & \\
\hline \hline
\end{tabular}


Observa-se na tabela 5.9 uma boa descrição da transição eletrônica para as duas formas da sonda, $\mathrm{MB}$ e $\mathrm{MBH}^{+}$, comparativamente aos valores experimentais apresentados. Analisando o valor absoluto da transição para o nosso melhor modelo $(\mathrm{X}+5 \mathrm{Sol}+495 \mathrm{CP})$, vemos que para a forma protonada $\mathrm{MBH}^{+}$o nosso resultado obtido com o funcional CAM-B3LYP está deslocado para o azul (comparado ao valor experimental) por cerca de $8 \mathrm{~nm}$, enquanto que o valor obtido com B3LYP está deslocado para o vermelho em $24 \mathrm{~nm}$. Para a forma neutra MB o deslocamento teórico $\rightarrow$ experimental é de $10 \mathrm{~nm}$ e $9 \mathrm{~nm}$ (ambos valores deslocados para menores comprimentos de onda), obtidos com os funcionais CAM-B3LYP e B3LYP, respectivamente. Com relação ao deslocamento na transição eletrônica devido à mudança forma protonada $\rightarrow$ neutra, nós observamos que o nosso resultado teórico apresenta um deslocamento de $71 \mathrm{~nm}$ e $40 \mathrm{~nm}$ (deslocamento para o vermelho), calculados com CAM-B3LYP e B3LYP. O deslocamento experimental é de $57 \mathrm{~nm}$, também deslocamento para o vermelho. Vemos que a tendência experimental é bem descrita pelo modelo teórico.

Os resultados teóricos mais próximos dos experimentais são os obtidos com CAMB3LYP e B3LYP com o modelo $\mathrm{X}+5 \mathrm{Sol}+495 \mathrm{CP}$.

Comparando os diferentes modelos de solvatação temos algumas considerações: (i) os modelos que envolvem apenas um cálculo quântico, $\mathrm{X}+\mathrm{PCM}$ (modelo contínuo) e $\mathrm{X}+\mathrm{ASEC}$ (modelo discreto), apresentam resultados próximos para $\mathrm{MBH}^{+}$e diferentes para MB. Comparativamente ao melhor modelo, $\mathrm{X}+5 \mathrm{Sol}+495 \mathrm{CP}$, o ASEC apresenta boa performance, com diferenças de aproximadamente $6 \mathrm{~nm}$ sempre para menor comprimento de onda (azul). Isso significa que a inclusão explícita de solventes sempre desloca a transição eletrônica para o vermelho, comparativamente a transição calculada apenas com o campo eletrostático do solvente. (ii) o modelo que inclui apenas as ligações de hidrogênio sem o campo eletrostático das demais moléculas do solvente, $\mathrm{X}+\mathrm{LH}$, apresenta os piores resultados comparativamente ao melhor modelo. Incluindo 
o campo eletrostático das demais moléculas, $\mathrm{X}+\mathrm{LH}+500 \mathrm{CP}$, existe sempre um deslocamento para menor comprimento de onda (azul). Isso significa que a inclusão do campo eletrostático sempre desloca a transição eletrônica para o azul, comparativamente a transição calculada sem o campo eletrostático do solvente. (iii) os modelos que incluem algumas moléculas do solvente e o campo eletrostático das demais, $\mathrm{X}+\mathrm{LH}+500 \mathrm{CP}$ e $\mathrm{X}+5 \mathrm{sol}+495 \mathrm{CP}$, apresentam valores muito próximos. Porém a inclusão explícita das ligações de hidrogênio, que envolve um grande custo computacional, representa uma pequena contribuição para a energia de transição (cerca de $3 \mathrm{~nm}$ ) comparativamente ao modelo que considera apenas o campo eletrostático do solvente, $\mathrm{X}+\mathrm{ASEC}$.

Sendo assim, concluímos que a maior contribuição do solvente na transição eletrônica da $\mathrm{MB}$ e $\mathrm{MBH}^{+}$em água vem da interação eletrostático deslocando a banda para menor comprimento de onda (azul) e da deformação da geometria, aumento do caráter benzoidal, deslocando a banda para maior comprimento de onda (vermelho). É interessante notar que comparativamente aos cálculos de vácuo (395 e 430 nm para $\mathrm{MBH}^{+}$e MB com B3LYP, ver tabela 5.1) os deslocamento são muito pequenos (395 e $435 \mathrm{~nm}$ para $\mathrm{MBH}^{+}$e MB com B3LYP, ver tabela 5.9 devido a compensação dos efeitos eletrostáticos e de deformação de geometria.

\section{Metanol}

Nós também realizamos o estudo em metanol, que é um solvente com constante dielétrica igual a 32.61. Os parâmetros para descrever metanol foram obtidos do campo de força OPLS-AA [107|. A simulação foi realizada com 1 molécula do soluto mais 1000 moléculas do solvente, a densidade inicial considerada para o metanol foi de $0.78 \mathrm{~g} / \mathrm{cm}^{3}$, à pressão de $1 \mathrm{~atm}$ e temperatura de $298 \mathrm{~K}$. Aqui, novamente foram realizados $60 \mathrm{mil}$ ciclos MC na termalização e 120 mil ciclos MC na etapa de produção. As simulações foram realizadas utilizando condições periódicas de contorno numa caixa cúbica de lado aproximadamente igual a $41.20 \AA$. Com relação à distribuição do solvente em 
torno do soluto, foi realizada a mesma análise descrita no tratamento com o solvente água. Na figura 5.6 apresentamos a função de distribuição radial de pares, G(r), entre o oxigênio do soluto e o oxigênio do solvente e tambem a RDF de mínima distância entre todos os átomos do soluto e os átomos do solvente.

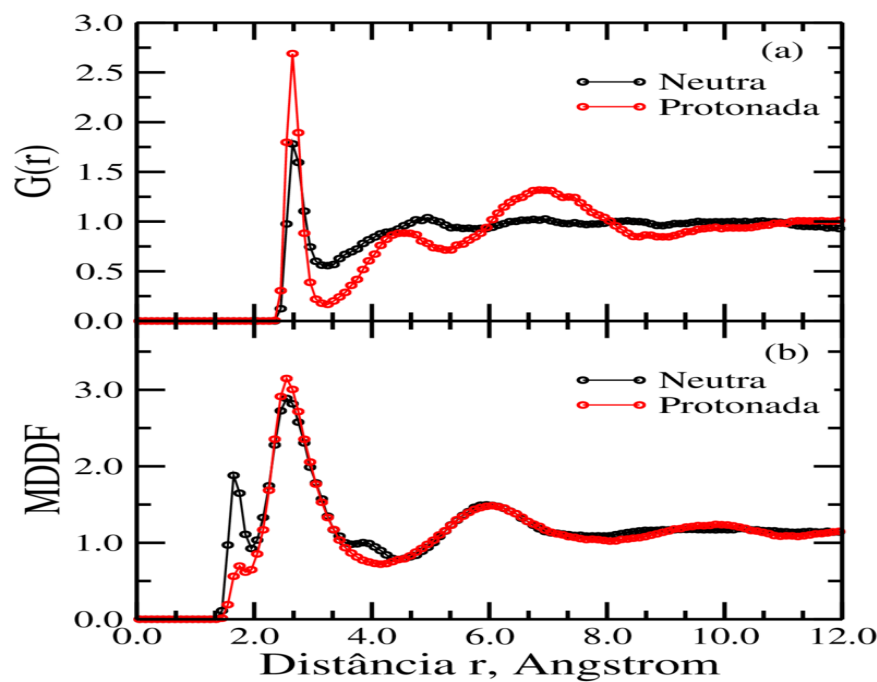

Figura 5.6: Função de distribuição radial de pares, solvente metanol. G(r) (a) e MDDF (b).

Observando a figura 5.6, vemos que para a $G_{O O}(r)$, as duas formas da sonda apresentam um primeiro pico com fim em 3.0 A. Nesta região estão as moléculas que podem formar ligação de hidrogênio. Com relação à curva MDDF, é possível observar alguns picos bem definidos para a sonda, um primeiro pico com fim em $2.0 \AA$, o segundo com fim em $4.0 \AA$, um terceiro com fim em $8.8 \AA$ e por fim, um quarto pico finalizando em 11.0 Å. A integração dessa função em cada um dos picos nos fornece o número de moléculas do solvente existente em cada camada de solvatação. Na tabela 5.10 fazemos um sumário com todos os resultados obtidos para as duas formas da sonda. Os picos caracterizam as camadas de solvatação, onde o $1^{\circ}$ pico equivale à microcamada de solvatação, o segundo pico é a primeira camada de solvatação e assim para os demais. Observa-se na figura 5.6 que a distribuição do solvente em torno das duas estruturas da sonda é similar. O limite da caixa possui 500 moléculas de solvente. 
Tabela 5.10: Número de moléculas de metanol distribuidos ao redor da sonda, obtidos pela integração da função de mínima distância, MDDF.

\begin{tabular}{lcccc}
\hline \hline Sonda & Microcamada & $1^{\circ}$ camada & $2^{\circ}$ camada & $3^{\circ}$ camada \\
\hline Distância $(\AA)$ & 2.0 & 4.0 & 8.8 & 11.0 \\
$\mathrm{MB}$ & 4 & 30 & 134 & 220 \\
$\mathrm{MBH}^{+}$ & 2 & 29 & 132 & 220 \\
\hline \hline
\end{tabular}

Geradas às configurações acessíveis ao líquido, nós selecionamos 120 configurações energeticamente descorrelacionadas as quais foram levadas ao cálculo quântico das transições eletrônicas. Na tabela 5.11 apresentamos os resultados obtidos para as duas formas da sonda considerando diferentes modelos para a solvatação do soluto. 
Tabela 5.11: Valores para o comprimento de onda da excitação eletrônica (em nm) da primeira transição, obtidos para diferentes modelos de solvatação em metanol. O cálculo foi realizado com o método TD-DFT, funcionais CAM-B3LYP e B3LYP com o conjunto de funções base 6-311 $+\mathrm{G}^{* *}$. Em parênteses está apresentado o valor para a força do oscilador da respectiva transição (OSC).

\begin{tabular}{ccccc}
\hline \hline Nível de QM & CAM-B3LYP & B3LYP & CAM-B3LYP & B3LYP \\
\hline $\mathrm{X}=$ & $\mathrm{MBH}^{+}$ & $\mathrm{MBH}^{+}$ & $\mathrm{MB}$ & $\mathrm{MB}$ \\
\hline Solvatação & $\lambda(\mathrm{OSC})$ & $\lambda(\mathrm{OSC})$ & $\lambda(\mathrm{OSC})$ & $\lambda(\mathrm{OSC})$ \\
$\mathrm{X}+\mathrm{PCM}$ & $380(1.26)$ & $415(1.17)$ & $466(1.53)$ & $478(1.44)$ \\
$\mathrm{X}+\mathrm{ASEC}$ & $365(1.14)$ & $395(1.04)$ & $430(1.35)$ & $442(1.24)$ \\
$\mathrm{X}+\mathrm{LH}$ & $407 \pm 3(1.24)$ & $442 \pm 2(1.01)$ & $461 \pm 1(1.59)$ & $582 \pm 3(0.02)$ \\
$\mathrm{X}+\mathrm{LH}+500 \mathrm{CP}$ & $369 \pm 1(1.18)$ & $399 \pm 1(1.08)$ & $432 \pm 1(1.45)$ & $447 \pm 1(1.34)$ \\
$\mathrm{X}+5$ Sol $+495 \mathrm{CP}$ & $370 \pm 1(1.15)$ & $402 \pm 1(1.05)$ & $433 \pm 1(1.43)$ & $449 \pm 4(1.33)$ \\
\hline & Solução ácida & & Solução alcalina & \\
\hline Experimental em metanol & $390 \pm 1(0.47)$ & & $485 \pm 1(0.68)$ & \\
\hline \hline
\end{tabular}


Observa-se na tabela 5.11 que obtemos uma descrição razoável para as formas da sonda estudadas comparativamente aos valores experimentais. Observa-se que para o resultado obtido com o funcional CAM-B3LYP o valor teórico para a forma protonada obtido com o nosso melhor modelo $(\mathrm{X}+5 \mathrm{Sol}+495 \mathrm{CP})$ está deslocado para o azul (menores comprimento de onda) em $20 \mathrm{~nm}$, comparado ao valor experimental, enquanto que o funcional B3LYP superestima o valor experimetal em $12 \mathrm{~nm}$ (deslocado para o vermelho). Com relação a forma neutra, o deslocamento teórico $\rightarrow$ experimental obtido com o funcional CAM-B3LYP é de $52 \mathrm{~nm}$, o funcional B3LYP descreve uma transição deslocada em $36 \mathrm{~nm}$, ambos os valores estão deslocados para o azul. A mudança da forma protonada $\rightarrow$ neutra sofrida pela sonda provoca um deslocamento na transição eletrônica. Este deslocamento experimental medido em metanol é de 95 nm, um deslocamento para o vermelho (maiores comprimentos de onda). O cálculo teórico nos fornece um deslocamento de $63 \mathrm{~nm}$ e $47 \mathrm{~nm}$ obtidos com os funcionais CAM-B3LYP e B3LYP, respectivamente. O deslocamento teórico descrito também é para o vermelho, o que mostra uma concordância na tendência entre os dois resultados.

Os resultados teóricos mais próximos dos experimentais são os obtidos com B3LYP com o modelo $\mathrm{X}+5 \mathrm{Sol}+495 \mathrm{CP}$ e CAM-B3LYP com o modelo $\mathrm{X}+\mathrm{PCM}$.

Comparando os diferentes modelos de solvatação temos considerações muito semelhantes as obtidas para água, mas algumas distintas: (i) os modelos $\mathrm{X}+\mathrm{PCM}$ e $\mathrm{X}+\mathrm{ASEC}$ apresentam resultados bem diferentes. Comparativamente ao melhor modelo, $\mathrm{X}+5 \mathrm{Sol}+495 \mathrm{CP}$, o ASEC apresenta boa performance, com diferenças de aproximadamente $6 \mathrm{~nm}$ sempre para menor comprimento de onda (azul). (ii) o modelo que inclui apenas as ligações de hidrogênio sem o campo eletrostático das demais moléculas do solvente, $\mathrm{X}+\mathrm{LH}$, apresenta os piores resultados comparativamente ao melhor modelo. Incluindo o campo eletrostático das demais moléculas, $\mathrm{X}+\mathrm{LH}+500 \mathrm{CP}$, existe sempre um deslocamento para menor comprimento de onda (azul). (iii) os modelos que incluem algumas moléculas do solvente e o campo eletrostático das demais, 
$\mathrm{X}+\mathrm{LH}+500 \mathrm{CP}$ e $\mathrm{X}+5 \mathrm{sol}+495 \mathrm{CP}$, apresentam valores muito próximos. Porém a inclusão explícita das ligações de hidrogênio representa uma pequena contribuição para a energia de transição (cerca de $3 \mathrm{~nm}$ ) comparativamente ao modelo que considera apenas o campo eletrostático do solvente, $\mathrm{X}+\mathrm{ASEC}$.

Sendo assim, concluímos que a maior contribuição do solvente na transição eletrônica da $\mathrm{MB}$ e $\mathrm{MBH}^{+}$em metanol também vem da interação eletrostático deslocando a banda para menor comprimento de onda (azul) e da deformação da geometria, aumento do caráter benzoidal, deslocando a banda para maior comprimento de onda (vermelho). É interessante notar que neste solvente interação eletrostática é menor que em água e as deformações geométricas são semelhantes (por terem sido geradas com PCM que é pouco sensível a diferença de constante dielétrica entre água e metanol, como mostrado na tabela 5.3). Assim, comparativamente aos cálculos de vácuo (395 e $430 \mathrm{~nm}$ para $\mathrm{MBH}^{+}$e MB com B3LYP, ver tabela 5.1) os deslocamento são para o azul (402 e $449 \mathrm{~nm}$ para $\mathrm{MBH}^{+}$e MB com B3LYP, ver tabela 5.9).

\section{Acetonitrila}

O solvente de menor polaridade que nós estudamos foi acetonitrila. A simulação foi realizada com 1 molécula do soluto mais 1000 moléculas do solvente, a densidade inicial considerada para a acetonitrila foi de $0.789 \mathrm{~g} / \mathrm{cm}^{3}$, à pressão de 1 atm e temperatura de $298 \mathrm{~K}$. Os parâmetros para descrever o solvente foram obtidos do campo de força OPLS-AA 108. Aqui, novamente foram realizados 60 mil ciclos MC na termalização e 120 mil ciclos MC na etapa de produção. As simulações foram realizadas utilizando condições periódicas de contorno numa caixa cúbica de lado aproximadamente igual a $43.82 \AA$. Com relação à distribuição do solvente em torno do soluto, nós analisamos a RDF de mínima distância, que está apresentada na figura 5.7 . 


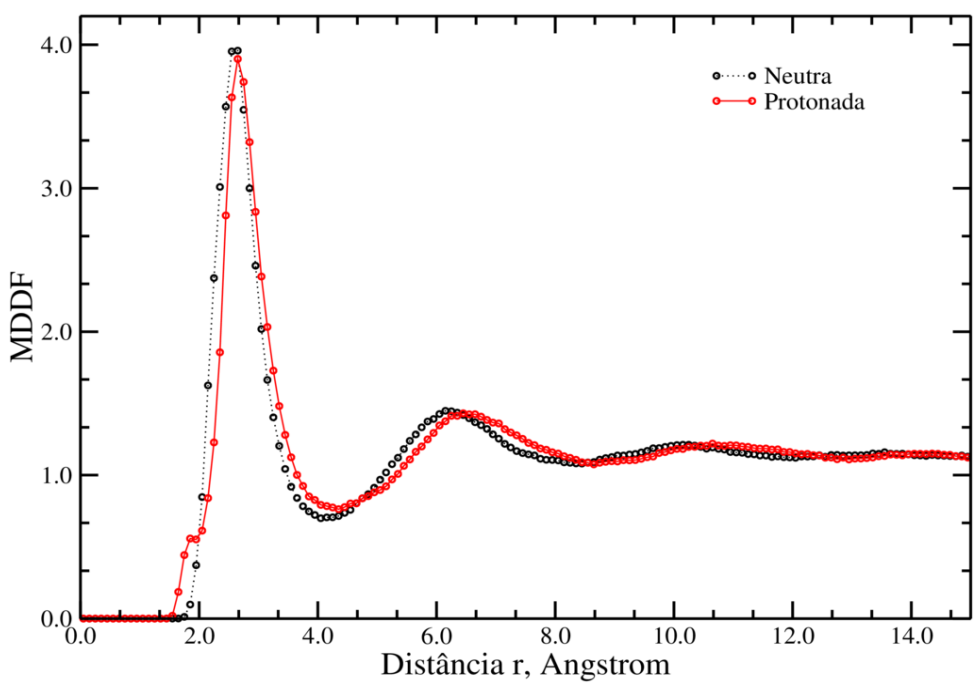

Figura 5.7: RDF de mínima distância, MDDF. Solvente acetonitrila.

Podemos observar na figura 5.7 que as duas formas (neutra/protonada) possuem uma distribuição do solvente bastante semelhante. Uma diferença observada entre as curvas é um pequeno ombro com fim em $2.0 \AA$ que está presente somente na curva referente à estrutura protonada. A integração desse pico nos dá o número de aproximadamente 1 molécula do solvente. As moléculas do solvente nesse região estariam disponíveis para formar ligação de hidrogênio soluto-solvente. A acetonitrila é um solvente aprótico, ela não doa prótons para formar ligação de hidrogênio com o soluto, porém ela contém um átomo eletronegativo, o nitrogênio $(\mathrm{N})$, o que possibilita que haja formação de LH onde o soluto é o doador do próton, e o solvente o aceitador. No nosso estudo, a única possibilidade para o soluto ser doador de próton é na forma protonada, o que explica o fato de não existir o mesmo pico na curva para a forma neutra. Por outro lado, nas duas curvas nós observamos três picos. Um primeiro pico com fim em $4.0 \AA$, um segundo pico com fim em $8.7 \AA$ e por fim, um terceiro pico discreto, terminando em 13.0 Å. Como mencionamos nas análises anteriores para os outros solventes, a integração desses picos nos dá o número de moléculas do solvente a uma dada distância r. Esses números estão apresentados na tabela 5.12. O limite 
da caixa de simulação possui 500 moléculas do solvente.

Tabela 5.12: Número de moléculas de acetonitrila obtido até o final de cada camada de solvatação.

\begin{tabular}{lccc}
\hline \hline Sonda & Microcamada & $1^{\circ}$ Camada & $2^{\circ}$ Camada \\
\hline Distância $(\AA)$ & 4.0 & 8.7 & 13.0 \\
$\mathrm{MB}$ & 26 & 112 & 271 \\
$\mathrm{MBH}^{+}$ & 27 & 113 & 273 \\
\hline \hline
\end{tabular}

Realizada a análise estrutura da distribuição do solvente em torno do soluto, nós selecionamos algumas configurações descorrelacionadas (total de 120 configurações) para realizar o cálculo da propriedade eletrônica de nosso interesse. As transições eletrônicas foram obtidas para as duas formas da sonda considerando dois métodos de funcionais distintos, CAM-B3LYP e B3LYP. Foram considerados diferentes modelos para mimetizar o efeito do meio, os resultados estão apresentados na tabela 5.13.

Com relação ao espectro de absorção da sonda em acetonitrila, nós vemos pela tabela 5.13 que para a transição eletrônica da molécula na estrutura protonada, há uma boa concordância com o resultado experimental. Com o funcional CAM-B3LYP e o modelo $\mathrm{X}+5 \mathrm{Sol}+495 \mathrm{CP}$ obtivemos um valor subestimado em $24 \mathrm{~nm}$, comparativamente ao valor experimental. Já o funcional B3LYP superestima esse valor em 12 nm. 
Tabela 5.13: Valores para o comprimento de onda da excitação eletrônica (em nm) da primeira transição, obtidos para diferentes modelos de solvatação em acetonitrila. O cálculo foi realizado com o método TD-DFT, funcionais CAM-B3LYP e B3LYP com o conjunto de funções base $6-311+\mathrm{G}^{* *}$. Em parênteses está apresentado o valor para a força do oscilador da respectiva transição (OSC).

\begin{tabular}{ccccc}
\hline \hline Nível de QM & CAM-B3LYP & B3LYP & CAM-B3LYP & B3LYP \\
\hline $\mathrm{X}=$ & MBH $^{+}$ & MBH $^{+}$ & MB & MB \\
\hline Solvatação & $\lambda($ OSC $)$ & $\lambda($ OSC $)$ & $\lambda($ OSC $)$ & $\lambda($ OSC $)$ \\
X+PCM & $368(1.21)$ & $409(1.10)$ & $466(1.53)$ & $480(1.44)$ \\
X + ASEC & $352(1.10)$ & $387(0.98)$ & $434(1.39)$ & $444(1.27)$ \\
X + 5Sol $+495 C P$ & $358 \pm 1(1.14)$ & $394 \pm 1(1.01)$ & $438 \pm 1(1.42)$ & $451 \pm 1(1.30)$ \\
\hline & Solução ácida & & Solução alcalina & \\
\hline Experimental em acetonitrila & $382 \pm 1(0.21)$ & & $567 \pm 1(0.65)$ & \\
\hline \hline
\end{tabular}


Os resultados teóricos mais próximos dos experimentais são novamente os obtidos com B3LYP com o modelo $\mathrm{X}+5 \mathrm{Sol}+495 \mathrm{CP}$ e CAM-B3LYP com o modelo $\mathrm{X}+\mathrm{PCM}$. Porém os resultados teóricos para a forma neutra estão muito deslocados comparativamente com os valores experimentais (cerca $100 \mathrm{~nm}$ ).

Comparando os diferentes modelos de solvatação temos considerações muito semelhantes as obtidas para metanol, mas algumas distintas: (i) os modelos $\mathrm{X}+\mathrm{PCM}$ e $\mathrm{X}+$ ASEC apresentam resultados próximos para $\mathrm{MBH}^{+}$e diferentes para MB. Comparativamente ao melhor modelo, $\mathrm{X}+5 \mathrm{Sol}+495 \mathrm{CP}$, o ASEC apresenta boa performance, com diferenças de aproximadamente $6 \mathrm{~nm}$ sempre para menor comprimento de onda (azul). (ii) A inclusão explícita de moléculas de solvente representa uma pequena contribuição para a energia de transição (cerca de $3 \mathrm{~nm}$ ) comparativamente ao modelo que considera apenas o campo eletrostático do solvente, $\mathrm{X}+\mathrm{ASEC}$.

Seguindo no estudo das estruturas que foram propostas para a sonda, nós apresentamos na seção a seguir a análise referente ao dímero $\mathrm{MB}_{2}$ em solução.

\subsection{Dímero de MB}

\subsubsection{Efeito Solvente: modelo contínuo}

No estudo dos dímeros em solução, nós otimizamos a geometria da sonda MB dimerizada na forma antiparalela embebida num meio contínuo polarizável PCM (solvente metanol). A geometria do dímero foi obtida com o nível de cálculo B3LYP/6$311+\mathrm{G}^{* *}$ na presença do respectivo solvente. Na figura 5.2 apresentamos uma ilustração do dímero antiparalelo estudado.

A energia de ligação do dímero em PCM foi obtida, cujo valor é de $35.1 \mathrm{kcal} / \mathrm{mol}$, o que mostra a estabilidade na formação deste complexo.

A caracterização do espectro eletrônico de absorção do dímero $\mathrm{MB}_{2}$ também foi 
feita. Nós calculamos a energia da transição eletrônica para os dímero em metanol (tratado através do modelo contínuo polarizável PCM) com dois funcionais DFT, CAM-B3LYP e B3LYP, com as funções base $6-311+\mathrm{G}^{* *}$. Na tabela 5.14 apresentamos os resultados obtidos. O valor para vácuo foi calculado na geometria otimizada na presença do solvente contínuo. Observa-se na tabela 5.14 que o dímero $\mathrm{MB}_{2}$ apresenta uma transição de mais baixa energia, na região de $600 \mathrm{~nm}$ com B3LYP, que possui uma força do oscilador baixa, e outra transição mais intensa na região de 420 nm, que aparece na mesma região da transição eletrônica para os monômero isolado, $430 \mathrm{~nm}$ (ver tabela 5.1).

Tabela 5.14: Valores para energia de transição $\left(\mathrm{em} \mathrm{cm}^{-1}\right)$, comprimento de onda (em nm) e força do oscilador (OSC) obtidos para o dímero $\mathrm{MB}_{2}$. Valores teóricos obtidos com TD-DFT usando os funcionais CAM-B3LYP e B3LYP e as funções base 6-311+ $\mathrm{G}^{* *}$.

\begin{tabular}{lllll}
\hline \hline Solvatação & B3LYP & \multicolumn{2}{c}{ CAM-B3LYP } \\
\hline \multirow{2}{*}{ Vácuo } & $\mathrm{E}(\mathrm{OSC})$ & $\lambda(\mathrm{nm})$ & $\mathrm{E}(\mathrm{OSC})$ & $\lambda(\mathrm{nm})$ \\
& $16181\left(10^{-4}\right)$ & 618 & $19455\left(10^{-4}\right)$ & 514 \\
& $23923(1.63)$ & 418 & $24510(2.46)$ & 408 \\
\hline PCM & $16978\left(10^{-4}\right)$ & 589 & $19608\left(10^{-4}\right)$ & 510 \\
& $22727(2.61)$ & 440 & $23095(2.77)$ & 433 \\
\hline \hline
\end{tabular}

Os resultados experimentais para a MB em metanol existentes na literatura são obtidos entre 483 e $490 \mathrm{~nm}[22,32,34,37$. Nesse sentido, aqui, o nosso melhor resultado para a descrição do espectro em metanol, comparativamente aos dados experimentais, é obtido com o modelo $\mathrm{MB}_{2}+\mathrm{PCM}$, calculado com o funcional CAM-B3LYP, apresentando o comprimento de onda de máxima absorção em $510 \mathrm{~nm}$. Entretanto, é importante ressaltar que a primeira transição calculada para o dímero $\mathrm{MB}_{2}$ varia de 
618 para $589 \mathrm{~nm}$ quando mudamos de vácuo para metanol. O dímero foi a única forma da sonda que conseguimos calcular transições nesta região de 650 a $550 \mathrm{~nm}$, onde é observada experimentalmente uma banda no espectro da sonda em solventes de baixa polaridade (573 nm para DMSO, $567 \mathrm{~nm}$ para acetonitrila e $615 \mathrm{~nm}$ para clorofórmio, os resultados experimentais serão discutidos com maior detalhe no capítulo 7 desta tese). Apesar da transição calculada ter baixa intensidade, é conhecido que estas transições ganham intensidade quando calculadas com modelos flexíveis da molécula considerando efeito de temperatura (ver exemplo na dissertação de V. W. Cruzeiro para monômeros e dímeros de ftalocianinas e porfirinas [109]. Portanto, acreditamos que em simulações de $\mathrm{MB}_{2}$ flexíveis em solução, esta primeira transição eletrônica terá uma força de oscilador maior.

A transição eletrônica associada ao dímero também é do tipo $\pi \rightarrow \pi^{*}$. A ilustração dos orbitais está apresentada na figura 5.8 .

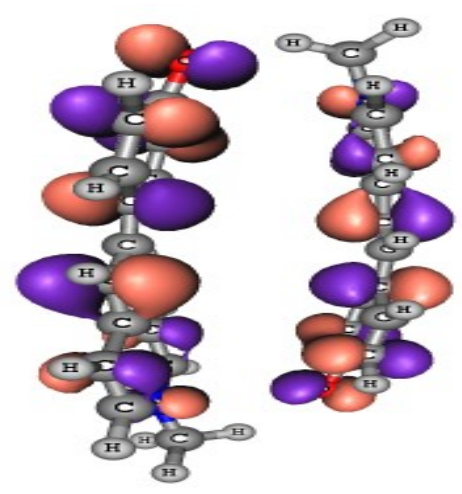

(a) HOMO

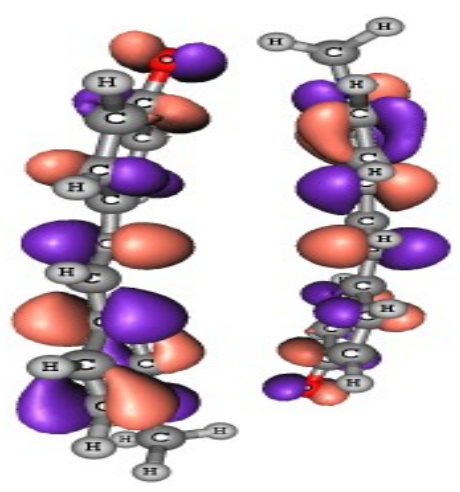

(b) LUMO

Figura 5.8: Ilustração dos orbitais moleculares envolvidos na transição eletrônica obtidos com TD-B3LYP $/ 6-311+\mathrm{G}^{* *}$ para o dímero de MB em metanol.

Caracterizada a formação do dímero num meio contínuo, nós partimos para o modelo em que as moléculas do solvente são descritas de maneira discreta e a característica estatística do líquido é levada em conta. Descrevemos essa etapa a seguir. 


\subsubsection{Efeito Solvente: modelo discreto}

Água

Nessa etapa do estudo do dímero $\mathrm{MB}_{2}$ em água, nós realizamos simulações clássicas usando o método Monte Carlo. A geometrias do dímero antiparalelo foi otimizada na presença do solvente, usando o modelo contínuo polarizável PCM, com o nível de cálculo B3LYP/6-311+G**. O procedimento para obtenção dos parâmetros do potencial de Lennard-Jones é similar ao descrito na seção 6.2. O conjunto de cargas utilizado foi o obtido para o monômero em água. As simulações foram realizadas no ensemble NPT. O número de partículas foi de 1 dímero $\mathrm{MB}_{2}$ e 1000 moléculas do solvente (água), à pressão de 1 atm e temperatura de 298 K. Na termalização foram gerados 60 mil ciclos MC e na etapa de produção foram gerados 120 mil ciclos MC. Verificamos que o dímero se separa rapidamente com cerca de 60 mil ciclos MC. Essa análise da separação foi feita visualizando as configurações salvas durante a simulação.

\section{Metanol}

Para o dímero $\mathrm{MB}_{2}$ em metanol, nós também realizamos simulações clássicas usando o método Monte Carlo. A geometrias do dímero antiparalelo foi otimizada na presença do solvente, usando o modelo contínuo polarizável PCM, com o nível de cálculo B3LYP/6-311+G**. O procedimento para obtenção dos parâmetros do potencial de Lennard-Jones é similar ao descrito na seção 6.2. O conjunto de cargas utilizado foi o obtido para o monômero em metanol. As simulações foram realizadas no ensemble NPT. O número de partículas foi de 1 dímero $\mathrm{MB}_{2}$ e 1000 moléculas do solvente (metanol), à pressão de 1 atm e temperatura de 298 K. Na termalização foram gerados 60 mil ciclos $\mathrm{MC}$ e na etapa de produção foram gerados 900 mil ciclos MC. Essa simulação foi muito mais longa que as anteriores para garantir a estabilidade do dímero que permaneceu ligado ao longo de toda simulação. Analisamos a energia de 
interação entre os monômeros, $\mathrm{U}_{11}$ apresentada na figura 5.9. Observa-se nesta figura que $\mathrm{U}_{11}$ é sempre negativa, ao longo da simulação de equilíbrio, e bastante forte com um valor médio de $-35.3 \pm 5.7 \mathrm{kcal} / \mathrm{mol}$. Isso mostra que a formação do dímero é mantida mesmo na presença das moléculas do solvente metanol.

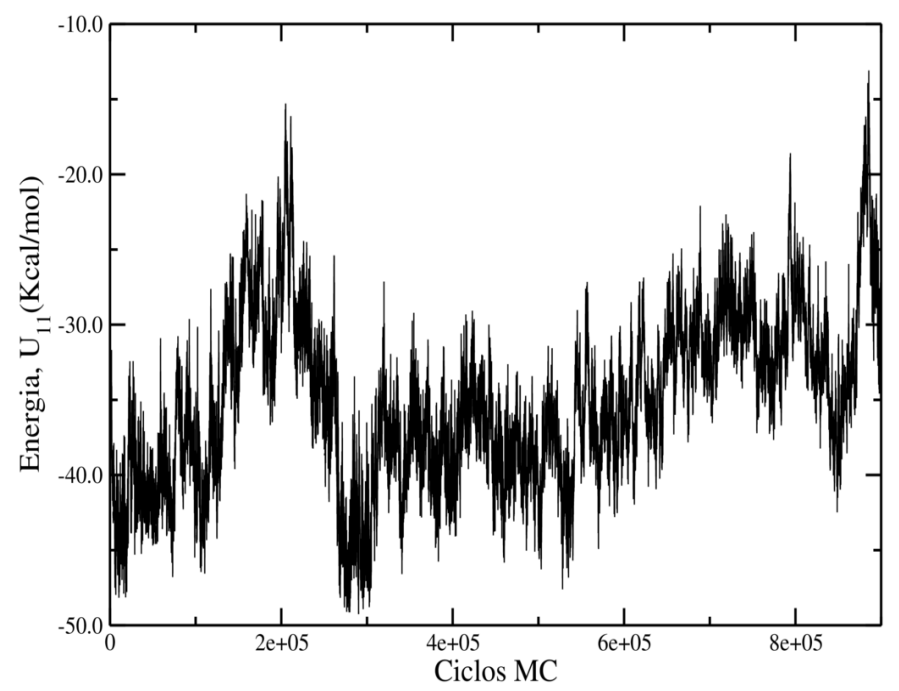

Figura 5.9: Energia de interação (em kcal/mol) do dímero $\mathrm{MB}_{2}$ ao longo da simulação $\mathrm{MC}$ em metanol.

Nós também podemos verificar outras propriedades para confirmar essa afirmação de que o dímero de $\mathrm{MB}_{2}$ é estável em metanol, como exemplo, a função de distribuição radial de pares, RDF. Na simulação clássica, nós partimos da configuração em que os monômeros estão próximos formando um dímero antiparalelo. O nosso interesse é verificar se a formação de dímero é estável ao longo da simulação em meio solvente. Para realizar essa análise nós obtivemos a função de distribuição radial de pares, RDF, entre os átomos do soluto. Fizemos isso entre os dois átomos de nitrogênio dos dois monômeros, $\mathrm{G}_{N-N}(r)$, e entre o nitrogênio de um monômero com o oxigênio do outro, $\mathrm{G}_{N-O}(r)$. Essas funções estão apresentadas na figura 5.10 . 


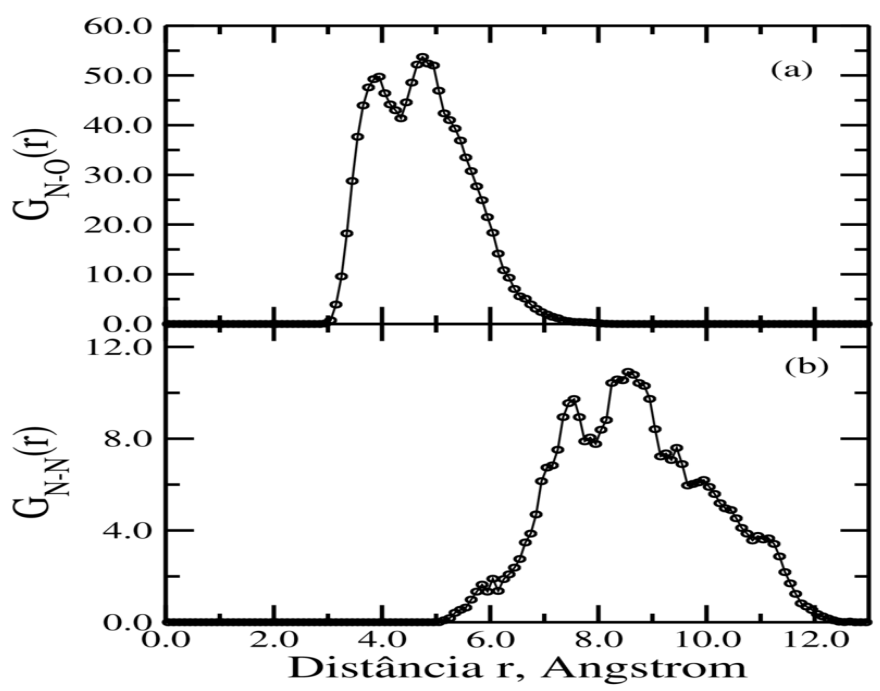

Figura 5.10: Função de distribuição radial de pares, G(r). Solvente metanol.

Observa-se na figura 5.10 um pico pronunciado em torno de $5 \AA$ para a $\mathrm{G}_{N-O}(r)$. Analisando a função $\mathrm{G}_{N-N}(r)$, observamos um pico em torno de $9 \AA$, caracterizando a proximidade dos monômeros formando o dímero.

Após a simulação, nós realizamos cálculos nas configurações geradas pela simulação clássica para obtermos a energia de transição correspondente ao dímero em solução de metanol. Para esse estudo, nós selecionamos 90 configurações descorrelacionadas obtidas da simulação e posteriormente realizamos cálculos quânticos para obtermos a propriedade eletrônica de interesse. Os valores para a energia de transição eletrônica foram obtidos com TD-DFT utilizando dois funcionais diferentes, B3LYP e CAMB3LYP, e o conjunto de funções base $6-311+\mathrm{G}^{* *}$. Na tabela 5.15 apresentamos os valores para a energia da transição eletrônica do dímero $\mathrm{MB}_{2}$ considerando diferentes modelos: vácuo, $\mathrm{PCM}$ e $500 \mathrm{CP}$. No modelo $500 \mathrm{CP}$ nós consideramos 500 moléculas do solvente metanol como cargas pontuais. Portanto, esse modelo é equivalente ao ASEC, apresentado anteriormente. Aqui o ASEC não pode ser usado pois como um monômero MB se move em relação ao outro, não é possível gerar a configuração eletrostática média. 
Tabela 5.15: Valores para energia de transição $\left(\mathrm{em}^{-1}\right)$, comprimento de onda (em nm) entre colchetes e força do oscilador da transição entre parênteses obtidos para o dímero $\mathrm{MB}_{2}$ para diferentes modelos de solvatação. Valores teóricos obtidos com TD-DFT usando os funcionais CAM-B3LYP e B3LYP e as funções base 6-311+G**.

\begin{tabular}{lrccc}
\hline \hline Solvatação & \multicolumn{2}{c}{ B3LYP } & \multicolumn{2}{c}{ CAM-B3LYP } \\
\hline \multirow{2}{*}{ Vácuo } & $\mathrm{E}(\mathrm{OSC})$ & $\lambda(n m)$ & $\mathrm{E}(\mathrm{OSC})$ & $\lambda(\mathrm{nm})$ \\
& $16181\left(10^{-4}\right)$ & 618 & $19455\left(10^{-4}\right)$ & 514 \\
& $23923(1.63)$ & 418 & $24510(2.46)$ & 408 \\
$\mathrm{MB}_{2}+\mathrm{PCM}$ & $16978\left(10^{-4}\right)$ & 589 & $19608\left(10^{-4}\right)$ & 510 \\
& $22727(2.61)$ & 440 & $23095(2.77)$ & 433 \\
$\mathrm{MB}_{2}+500 \mathrm{CP}$ & $16143 \pm 125\left(10^{-4}\right)$ & $621 \pm 5$ & $21031 \pm 84\left(10^{-4}\right)$ & $476 \pm 2$ \\
& $23976 \pm 183(1.91)$ & $417 \pm 3$ & $23222 \pm 126(0.09)$ & $431 \pm 2$ \\
\hline \hline
\end{tabular}


Observa-se na tabela 5.15 que a mudança vácuo $\rightarrow$ PCM desloca a energia para maiores valores, cerca de $800 \mathrm{~cm}^{-1}$ para o funcional B3LYP e $153 \mathrm{~cm}^{-1}$ para o funcional CAM-B3LYP. Isso é observado para a transição de mais baixa energia. Já para a mudança vácuo $\rightarrow 500 \mathrm{CP}$ observa-se o seguinte comportamento: um deslocamento de $-38 \mathrm{~cm}^{-1}$ para o funcional B3LYP e um deslocamento de $1576 \mathrm{~cm}^{-1}$ para o funcional CAM-B3LYP. Portanto, para o funcional B3LYP não há mudança significativa na inclusão do solvente como modelo contínuo ou discreto. Porém com as mesmas configuração, o funcional CAM-B3LYP apresenta uma mudanças de cerca de $35 \mathrm{~nm}$ para o azul indo do modelo contínuo para o discreto. Comparativamente aos dados experimentais que mostram que o valor para o comprimento de onda de máxima absorção da sonda MB em metanol variam entre $483 \mathrm{~nm}$ e $490 \mathrm{~nm}$, nós temos que o melhor resultado apresentado aqui foi em $476 \mathrm{~nm}$, obtido com $\mathrm{MB}_{2}+500 \mathrm{CP}$ e o funcional CAM-B3LYP.

\subsection{Determinação da constante de acidez}

Nesta seção nós apresentaremos o estudo realizado para a obtenção o valor de $\mathrm{pK}_{a}$ associado ao processo de protonação/desprotonação da sonda MB. Esse estudo foi realizado considerando dois solventes diferentes, água e metanol.

O valor de $\mathrm{pK}_{a}$ pode ser entendido como uma medida da interação solutosolvente. Dependendo de quão forte é a interação do solvente com a forma ácida/protonada $\left(H A^{+}\right)$de uma substância ou com a sua forma base conjugada/desprotonada $(A)$, o equilíbrio da reação de dissociação pode ser deslocado para o lado ácido ou para o lado da base conjugada. Ilustramos esse comportamento na equação 5.1

$$
H A_{(s o l)}^{+} \stackrel{K_{a}}{\rightleftarrows} A_{(s o l)}+H_{(s o l)}^{+}
$$

onde $K_{a}$ é a constante de dissociação do ácido $H A^{+}$e está relacionada ao $\mathrm{pk}_{a}$ da 
seguinte forma:

$$
p K_{a}=-\log K_{a}
$$

A determinação teórica do valor de $\mathrm{pK}_{a}$ de uma dada substância pode ser feita por diferentes metodologias [110], onde diferentes ciclos termodinâmicos podem ser usados para obter o valor de $\mathrm{pK}_{a}$ de uma dada substâncias. Aqui usaremos o ciclo ilustrado no Esquema 5.1 que é baseado na reação ácido-base em água 111.

$$
\begin{array}{cccccc}
\mathrm{MBH}^{+}{ }_{(\mathrm{g})}+ & \mathrm{H}_{2} \mathrm{O}_{(\mathrm{g})} & \stackrel{\Delta G_{g}}{\longrightarrow} & \mathrm{MB}_{(\mathrm{g})}+ & \mathrm{H}_{3} \mathrm{O}_{(\mathrm{g})}^{+} \\
\downarrow_{\Delta G_{\text {solv }}\left(M B H^{+}\right)} & \downarrow \Delta G_{\text {solv }}\left(H_{2} O\right) & \downarrow \Delta G_{\text {solv }}(M B) & \downarrow \Delta G_{\text {solv }}\left(H_{3} O^{+}\right) \\
\mathrm{MBH}_{(\mathrm{sol})}^{+}+ & \mathrm{H}_{2} \mathrm{O}_{(\mathrm{sol})} & \stackrel{\Delta G_{\text {s }} l}{\longrightarrow} & \mathrm{MB}_{(\text {sol })} & + & \mathrm{H}_{3} \mathrm{O}_{(\text {sol })}^{+}
\end{array}
$$

Esquema 5.1: Ciclo termodinâmico usado no cálculo da diferença de energia livre da reação ácido-base da sonda MB em água.

Baseado nas diferenças de energia livre obtidas pelo ciclo termodinâmico apresentado no esquema 5.1, é possível obter o $p K_{a}$ usando a seguinte equação

$$
p K_{a}=\frac{\Delta G_{(s o l)}}{R T \ln (10)}-\log \left[H_{2} O\right]
$$

Uma dedução da equação 5.3 pode ser encontrada na referência [111. Nesta equação, o termo $\left[\mathrm{H}_{2} \mathrm{O}\right]$ se refere a concentração da água, que para o estado padrão da água, pressão de 1 atm e temperatura de $298 \mathrm{~K}$, é de $55 \mathrm{~mol} / \mathrm{L}$. A energia livre da reação em solução, $\Delta G_{(s o l)}$, contém contribuições das diferenças de energia livre em fase gasosa, $\Delta G_{(g)}$, e das diferenças da energia livre de solvatação, $\Delta \Delta G_{(s o l v)}$.

$$
\Delta G_{\text {sol }}=\Delta G_{(g)}+\Delta \Delta G_{(\text {solv })}
$$


onde a variação da energia livre de fase gasosa é dada por

$$
\Delta G_{(g)}=G_{(g)}(M B)+G_{(g)}\left(H_{3} O^{+}\right)-G_{(g)}\left(M B H^{+}\right)-G_{(g)}\left(H_{2} O\right)
$$

e a variação da energia livre de solvatação, que baseado na definição de Ben-Naim [112, 113 representa a energia livre necessária para transferir $1 \mathrm{~mol}$ do soluto numa hipotética condição de gas ideal para uma solução ideal com diluição infinita também a 1 mol, é dada por

$$
\Delta \Delta G_{(s o l v)}=\Delta G_{(s o l v)}(M B)+\Delta G_{(s o l v)}\left(H_{3} O^{+}\right)-\Delta G_{(s o l v)}\left(M B H^{+}\right)-\Delta G_{(s o l v)}\left(H_{2} O\right)
$$

Agora, todos os termos presentes nas equações 5.5 e 5.6 podem ser calculados usando métodos quânticos com a molécula em vácuo e em solução, respectivamente. Desta forma, podemos estimar o valor de $\mathrm{pK}_{a}$ para uma dada molécula em solução aquosa.

Os cálculos de fase gasosa (vácuo) foram realizados utilizando o método DFT com o funcional B3LYP e o conjunto de funções base $6-311+\mathrm{G}^{* *}$, na tabela 5.16 apresentamos as contribuições para a energia livre em fase gasosa obtida para os constituintes da reação. Os valores são apresentados em kcal $/ \mathrm{mol}$. 
Tabela 5.16: Energia livre de fase gasosa para os constintuintes da reação ácido-base com a água em unidades atômica (u.a) e variação da energia livre, $\Delta G_{g}$, em $(\mathrm{kcal} / \mathrm{mol})$.

\begin{tabular}{cc}
\hline \hline Energia livre em gás, $G_{g}$ & $\mathrm{E}(\mathrm{u} . \mathrm{a})$ \\
\hline$G_{g}\left(\mathrm{H}_{2} \mathrm{O}\right)$ & -76.46 \\
$G_{g}\left(\mathrm{H}_{3} \mathrm{O}^{+}\right)$ & -76.71 \\
$G_{g}\left(\mathrm{OH}^{-}\right)$ & -75.83 \\
$G_{g}\left(\mathrm{MBH}^{+}\right)$ & -671.44 \\
$G_{g}(\mathrm{MB})$ & -671.23 \\
\hline$\Delta G_{g}\left(2 \mathrm{H}_{2} \mathrm{O} \rightarrow \mathrm{OH}+\mathrm{H}_{3} \mathrm{O}^{+}\right)$ & $226.01(\mathrm{kcal} / \mathrm{mol})$ \\
$\Delta G_{g}\left(\mathrm{MBH}^{+}+\mathrm{H}_{2} \mathrm{O} \rightarrow \mathrm{MB}+\mathrm{H}_{3} \mathrm{O}^{+}\right)$ & $0.36(\mathrm{u} . \mathrm{a})$ \\
& $0.15(\mathrm{kcal} / \mathrm{mol})$ \\
\hline \hline
\end{tabular}


Agora como saberemos se o método utilizado nos fornece um bom resultado para a energia livre em fase gasosa? Uma boa maneira para estimarmos isso é comparando os nossos valores com resultados já obtidos por outros autores. Por exemplo, na literatura há resultados experimentais mostrando que a energia associada ao processo de dissociação da água, $2 \mathrm{H}_{2} \mathrm{O} \rightarrow \mathrm{OH}^{-}+\mathrm{H}_{3} \mathrm{O}^{+}$, é de $226 \mathrm{kcal} / \mathrm{mol}$ [114,115], portanto, o nosso valor de $226.01 \mathrm{kcal} / \mathrm{mol}$ obtido com B3LYP $/ 6-311+\mathrm{G}^{* *}$, está em bom acordo com o valor já descrito na literatura. Com relação aos valores obtidos para a sonda nós não teremos como fazer essa mesma análise, pois não há outros resultados reportados na literatura.

Em seguida nós obtivemos os valores para a energia livre de solvatação, $\Delta G_{\text {sol }}$. Nessa etapa o solvente foi considerado através do modelo contínuo PCM, usando a aproximação PCM-UAHF [116]. Neste modelo contínuo para o solvente, a energia de solvatação pode ser decomposta em 4 diferentes contribuições:

$$
\Delta G_{(\text {solv })}=\Delta G_{\text {eletr }}+\Delta G_{\text {cav }}+\Delta G_{\text {rep }}+\Delta G_{\text {disp }}
$$

O termo eletrostático, $\Delta G_{\text {eletr }}$, é o que fornece uma maior contribuição para a energia de solvatação. Os termos de cavitação $\left(\Delta G_{c a v}\right)$, repulsão $\left(\Delta G_{r e p}\right)$ e dispersão $\left(\Delta G_{d i s p}\right)$, compõem a contribuição não eletrostática para a energia de solvatação. Na tabela 5.19 apresentamos os resultados obtidos separados nas contribuições eletrostáticas e não-eletrostáticas. O nível de cálculo utilizado foi $\mathrm{HF} / 6-31+\mathrm{G}^{*}$, que é o método recomendado para se obter uma correta descrição na energia livre de solvatação utilizando a aproximação PCM-UAHF. 
Tabela 5.17: Energia livre de solvatação para os constintuintes da reação ácido-base com a água.

\begin{tabular}{ccc}
\hline \hline$\Delta G_{\text {solv }}$, Contribuição não-eletrostática & $\mathrm{MB}$ & $\mathrm{MBH}^{+}$ \\
$\Delta G_{\text {solv }}$, Contribuição eletrostática & 3.55 & 3.26 \\
$\Delta G_{\text {solv }}$, Total & -24.64 & -52.52 \\
& -21.09 & \\
$\Delta G_{\text {solv }}\left(\mathrm{H}_{2} \mathrm{O}\right)$ & Experimental |114, 117, 118.27 & \\
$\Delta G_{\text {solv }}\left(\mathrm{H}_{3} \mathrm{O}^{+}\right)$ & -6.32 & \\
\hline \hline
\end{tabular}

Agora com os valores para a energia livre de fase gasosa (tabela 5.16) e com os valores para a energia livre de solvatação (tabela 5.17), nós podemos obter a energia livre da reação em solução, $\Delta G_{(s o l)}$, apresentado na equação 5.4 . Nós apresentamos os valores obtidos na tabela 5.18 .

Tabela 5.18: Contribuições para o cálculo do $\mathrm{pK}_{a}$.

\begin{tabular}{cc}
\hline \hline Contribuições & $\mathrm{E}(\mathrm{kcal} / \mathrm{mol})$ \\
$\Delta \Delta G_{\text {solv }}(\mathrm{MB})$ & 28.18 \\
$\Delta \Delta G_{\text {solv }}\left(\mathrm{H}_{2} \mathrm{O}\right)$ & -103.88 \\
$\Delta G_{\text {solv }}=\Delta \Delta G_{\text {solv }}(\mathrm{MB})+\Delta \Delta G_{\text {solv }}\left(\mathrm{H}_{2} \mathrm{O}\right)$ & -75.70 \\
$\Delta G_{g}$ & 94.95 \\
$\Delta G_{\text {sol }}$ & 19.25 \\
$\mathrm{pK}_{a}$ & 12.4 \\
\hline \hline
\end{tabular}


Onde $\Delta \Delta G_{\text {solv }}(\mathrm{MB})=\Delta G_{\text {solv }}(\mathrm{MB})-\Delta G_{\text {solv }}\left(\mathrm{MBH}^{+}\right)$, e $\Delta \Delta G_{\text {solv }}\left(\mathrm{H}_{2} \mathrm{O}\right)=\Delta G_{\text {solv }}\left(\mathrm{H}_{3} \mathrm{O}^{+}\right)-$ $\Delta G_{\text {solv }}\left(\mathrm{H}_{2} \mathrm{O}\right)$. Observa-se na tabela 5.18 que os nossos resultados nos fornecem um valor de $19.25 \mathrm{kcal} / \mathrm{mol}$ para a energia livre da reação, $\Delta G_{\text {sol }}$, e substituindo esse valor na equação 5.3, nós obtivemos um valor de 12.4 para o $\mathrm{pK}_{a}$ da $\mathrm{MB}$ em água.

Em seguida, nós também realizamos o mesmo estudo para determinar o $\mathrm{pK}_{a p}$ da sonda em solução de metanol. Neste caso, no esquema 5.1 e nas equações 5.5 e 5.6 a molécula de água, $\mathrm{H}_{2} \mathrm{O}$, é substituido pela molécula de metanol, $\mathrm{CH}_{3} \mathrm{OH}$, e a molécula de água protonada, $\mathrm{H}_{3} \mathrm{O}^{+}$, pela molécula de metanol protonada, $\mathrm{CH}_{3} \mathrm{OH}_{2}^{+}$. Novamente é válida toda a discussão feita para o caso da água. Nós devemos obter os valores para a energia livre em fase gasosa e a energia livre de solvatação em metanol. A energia livre da reação, $\Delta G_{(s o l)}$, é obtida considerando a equação 5.4. Na tabela 5.19 apresentamos os valores para a fase gasosa obtidos com B3LYP $/ 6-311+\mathrm{G}^{* *}$.

Tabela 5.19: Energia livre de fase gasosa para os constintuintes da reação ácido-base com o metanol em unidades atômica (u.a) e variação da energia livre, $\Delta G_{g}$, em (kcal $/ \mathrm{mol}$ ).

\begin{tabular}{cc}
\hline \hline Energia livre em gás, $G_{g}$ & $\mathrm{E}($ u.a $)$ \\
\hline$G_{g}\left(\mathrm{CH}_{3} \mathrm{OH}\right)$ & -115.74 \\
$G_{g}\left(\mathrm{CH}_{3} \mathrm{OH}_{2}^{+}\right)$ & -116.02 \\
$G_{g}\left(\mathrm{CH}_{3} \mathrm{O}^{-}\right)$ & -115.12 \\
$G_{g}\left(\mathrm{MBH}^{+}\right)$ & -671.44 \\
$G_{g}(\mathrm{MB})$ & -671.23 \\
\hline$\Delta G_{g}\left(2 \mathrm{CH}_{3} \mathrm{OH} \rightarrow \mathrm{CH}_{3} \mathrm{O}^{-}+\mathrm{CH}_{3} \mathrm{OH}_{2}^{+}\right)$ & $207.84(\mathrm{kcal} / \mathrm{mol})$ \\
$\Delta G_{g}\left(\mathrm{MBH}^{+}+\mathrm{CH}_{3} \mathrm{OH} \rightarrow \mathrm{MB}+\mathrm{CH}_{3} \mathrm{OH}_{2}^{+}\right)$ & $0.33(\mathrm{u} . \mathrm{a})$ \\
\hline
\end{tabular}


Obtida a energia livre de fase gasosa, nós realizamos o cálculo da energia livre de solvatação em metanol. Similar ao estudo na água, o solvente foi tratado através do modelo contínuo PCM-UAHF com o nível de cálculo HF/6-31+G*. Os resultados obtidos estão apresentados na tabela 5.20 , onde novamente fazemos a separação entre contribuições eletrostáticas e não-eletrostáticas.

Tabela 5.20: Energia livre de solvatação para os constintuintes da reação ácido-base em metanol.

\begin{tabular}{ccc}
\hline \hline & $\mathrm{MB}$ & $\mathrm{MBH}^{+}$ \\
$\Delta G_{\text {solv }}$, Contribuição não-eletrostática & -1.01 & -1.30 \\
$\Delta G_{\text {solv }}$, Contribuição eletrostática & -23.70 & -51.12 \\
$\Delta G_{\text {solv }}$, Total & -24.71 & -52.42 \\
\hline$\Delta G_{\text {solv }}\left(\mathrm{CH}_{3} \mathrm{OH}\right)$ & -4.86 & \\
$\Delta G_{\text {solv }}\left(\mathrm{CH}_{3} \mathrm{OH}_{2}^{+}\right)$ & -91.41 & \\
\hline \hline
\end{tabular}

Agora, utilizando os resultados apresentados nas tabelas 5.19 e 5.20 , nós podemos obter o valor para a energia livre da reação em metanol, $\Delta G_{(s o l)}$. Esse resultado e os demais termos necessários para o cálulo do $\mathrm{pK}_{a p}$ estão apresentados na tabela 5.21 . 
Tabela 5.21: Contribuições para o cálculo do $\mathrm{pK}_{a}$.

\begin{tabular}{cc}
\hline \hline Contribuições & $\mathrm{E}(\mathrm{kcal} / \mathrm{mol})$ \\
$\Delta \Delta G_{\text {solv }}(\mathrm{MB})$ & 27.71 \\
$\Delta \Delta G_{\text {solv }}\left(C H_{3} O H\right)$ & -86.55 \\
$\Delta G_{\text {solv }}=\Delta \Delta G_{\text {solv }}(\mathrm{MB})+\Delta \Delta G_{\text {solv }}\left(\mathrm{CH}_{3} O H\right)$ & -58.84 \\
$\Delta G_{g}$ & 79.16 \\
$\Delta G_{\text {sol }}$ & 20.32 \\
$\mathrm{pK}_{a p}$ & 13.5 \\
\hline \hline
\end{tabular}

Utilizando a equação 5.3 , com a modificação de que agora a reação ocorre em metanol e, portanto, o fator de correção será $\log \left[\mathrm{CH}_{3} \mathrm{OH}\right]$, nós podemos obter o $\mathrm{pK}_{a p}$ para a sonda em metanol. Anotando que a concentração de metanol, $\left[\mathrm{CH}_{3} \mathrm{OH}\right]$, no estado padrão é de $24.5 \mathrm{~mol} / \mathrm{L}$, nós obtivemos um valor de $\mathrm{pK}_{a}$ igual a 13.5 .

Posteriormente iremos comparar os nossos valores para o $\mathrm{pK}_{a}$ teórico com os valores obtidos experimentalmente que serão apresentados no capítulo 7 . 


\section{Capítulo 6}

\section{Derivados da Merocianina de Brooker: Resultados teóricos}

Neste capítulo apresentaremos os resultados dos estudos teóricos realizados nos derivados bromatados da sonda MB. Estudamos o efeito do solvente no espectro de absorção analisando diferentes solventes e a possibilidade de agregação.

\subsection{Molécula Isolada}

Nesta seção apresentamos o estudo realizado para obtenção das propriedades estruturais e eletrônicas das moléculas isoladas. Como foi mencionado anteriormente, há dificuldades na descrição teórica do comprimento de máxima absorção para algumas merocianinas em solventes pouco polares. Uma das causas no erro da descrição pode estar relacionada com a estrutura do estado fundamental das sondas. Para investigar essa hipótese nós geramos três possibilidades para a estrutura das moléculas: forma neutra (quinoidal), zwiteriônica (benzoidal) e protonada. Assim, realizamos cálculos quânticos para otimização das geometrias correspondentes a cada uma das formas e estudamos os respectivos espectros de absorção e momentos de dipolo. 


\subsubsection{Geometrias}

As moléculas estudadas estão ilustradas na figura 6.1. O primeiro passo nessa etapa foi a obtenção das geometrias moleculares que corresponderiam à um mínimo de energia. Para isso nós realizamos cálculos quânticos usando o funcional de densidade B3LYP 66, 71, com o conjunto de funções base 6-311+G**.

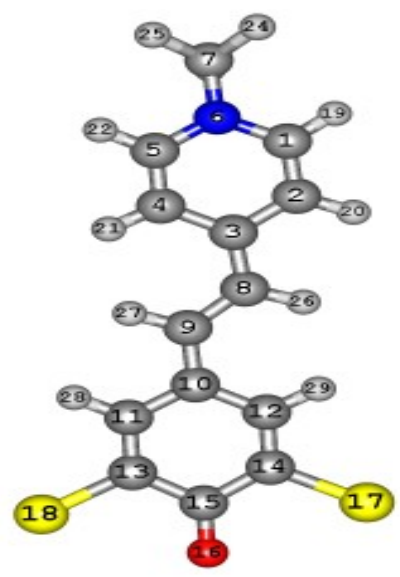

(a) $\mathrm{MePMBr}_{2}$

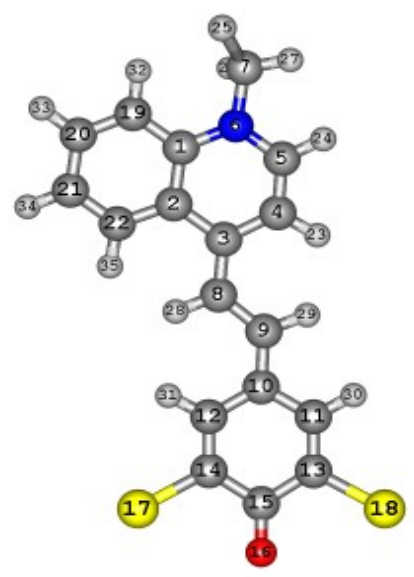

(b) $\mathrm{MeQMBr}_{2}$

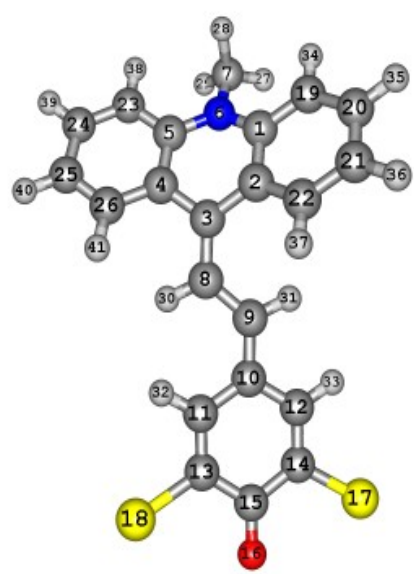

(c) $\mathrm{MeAMBr}_{2}$

Figura 6.1: Ilustração das geometrias das sondas estudadas e a numeração dos átomos.

Para obter o momento de dipolo das moléculas nós realizamos cálculos com teoria de perturbação de segunda ordem, MP2 [57,58], utilizando o ajuste do potencial eletrostático com o procedimento CHELPG 83 e com o mesmo conjunto de funções base usado para otimização da geometria. Na tabela 6.1 apresentamos algumas informações estruturais e eletrostáticas das moléculas. 
Tabela 6.1: Valores de energia $(\mathrm{kcal} / \mathrm{mol})$, momento de dipolo (D), carga atômica (e), comprimento da ligação $(\AA)$ e ângulos diedros $\left(^{\circ}\right)$ para as sondas solvatocrômicas otimizadas na conformação trans com B3LYP/6-311+ $\mathrm{G}^{* *}$ e dipolo com MP2/6-311+ $\mathrm{G}^{* *}$.

\begin{tabular}{cccc}
\hline \hline Propriedades & trans-MePMBr2 & trans-MeQMBr2 & trans-MeAMBr2 \\
\hline Energia (kcal/mol) & -3651170.060 & -3747606.060 & -3844037.250 \\
Dipolo (D) & 17.418 & 15.594 & 11.618 \\
Carga (N6) & 0.102 & -0.075 & -0.337 \\
Carga (C8) & -0.359 & -0.215 & -0.092 \\
Carga (C9) & -0.069 & -0.142 & -0.136 \\
Carga (O16) & -0.544 & -0.538 & -0.529 \\
Carga(C15) & 0.645 & 0.645 & 0.683 \\
R(C8-C9) & 1.400 & 1.403 & 1.414 \\
R(C15-O16) & 1.223 & 1.222 & 1.220 \\
$\Phi(\mathrm{C} 4-\mathrm{C} 3-\mathrm{C} 8-\mathrm{C} 9)$ & 0.094 & -0.001 & 166.767 \\
$\Phi(\mathrm{C} 3-\mathrm{C} 8-\mathrm{C} 9-\mathrm{C} 10)$ & 179.911 & 179.999 & 178.161 \\
$\Phi(\mathrm{C} 8-\mathrm{C} 9-\mathrm{C} 10-\mathrm{C} 12)$ & -0.044 & 0.002 & 174.322 \\
\hline \hline
\end{tabular}


$\mathrm{Na}$ análise geométrica observamos que as sondas trans- $\mathrm{MePMBr}_{2}$ e trans- $\mathrm{MeQMBr}_{2}$ possui geometrias planares enquanto que sonda trans-MeAMBr 2 não é planar, essa informação é obtida na tabela 6.1 através do diedro, $\Phi(\mathrm{C} 4-\mathrm{C} 3-\mathrm{C} 8-\mathrm{C} 9)$, e está em boa concordância com outros resultados teóricos $[30 \mid$. Observa-se que naturalmente o estrutura das sondas assumem o isômero trans, classificado com relação ao diedro $\Phi(\mathrm{C} 3$ C8-C9-C10), que é aproximadamente $180^{\circ}$ para as três sondas. Observa-se também que ao aumentarmos o número de anéis aromáticos há um aumento no comprimento da ligação C8-C9 o que indicaria um pequeno aumento no caráter da ligação simples. Por outro lado, ocorre uma pequena diminuição da ligação C15-O16, o que indica que o acréscimo de anéis aromáticos aumenta o caráter quinoidal da molécula. Com relação ao momento de dipolo ocorre uma diminuição com o aumento dos anéis aromáticos. Uma outra possibilidade para a geometria do estado fundamental das moléculas estudadas foi a forma cis (ou syn), aqui o isômerismo cis-trans foi analisado com relação ao diedro formado pela cadeia de átomos C3-C8-C9-C10. Na figura 6.2 apresentamos a ilustração das geometrias da conformação cis, após o processo de otimização.

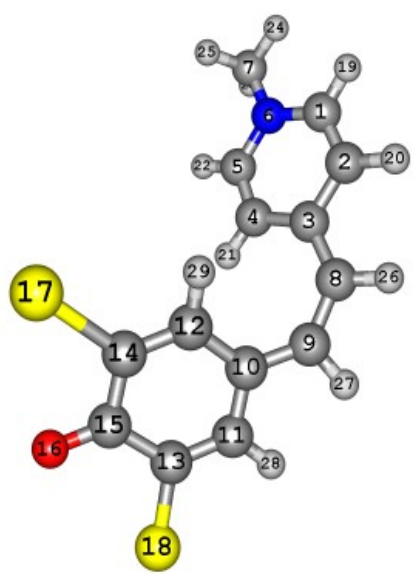

(a) cis-MePMBr 2

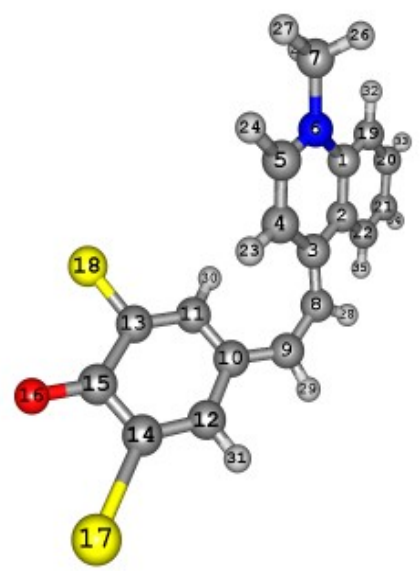

(b) cis- $\mathrm{MeQMBr}_{2}$

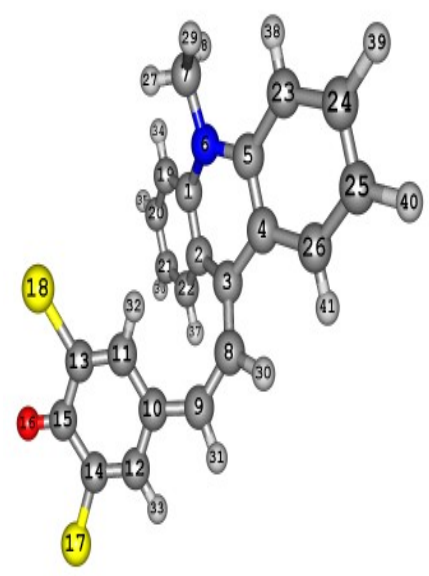

(c) cis-MeAMBr 2

Figura 6.2: Ilustração das geometrias das sondas estudadas na forma cis.

Na tabela 6.2 apresentamos algumas informações estruturais e eletrônicas das 
sondas na conformação cis. Observa-se que o comportamento para o comprimento das ligações C8-C9 e C15-C16, é mesmo observado para as formas trans quando há o aumento no número de anéis aromáticos. Isso também é verificado para o momento de dipolo das moléculas. Pela análise das energias eletrônicas observa-se que a forma trans é a mais estável e também possui o maior momento de dipolo o que irá contribuir para uma maior estabilização dessa conformação em meios polares.

No estudo das sondas isoladas nós analisamos algumas propriedades estruturais das conformações cis e trans para a forma neutra (ou quinoidal), porém estudos mostram [37,129] que as estruturas eletrônicas das merocianinas podem ser descritas como um híbrido de ressonância entre as formas quinoidal (C8-C9 formando uma ligação simples) e zwiteriônica (ou benzoidal) (C8-C9 formando uma dupla ligação), e que uma perturbação externa, como a interação com o solvente, pode modificar as contribuições de cada uma das formas, sendo estas contribuições sensíveis à polaridade do solvente. Nós observamos que a geometria obtida pelo cálculo quântico, conforme descrito anteriormente, sem impor nenhum vínculo, se apresenta na forma neutra. Portanto, para obter a forma zwiteriônica nós realizamos um procedimento já utilizado na literatura [97, que é aproximar um próton $\mathrm{H}^{+}$, no nosso caso, ao átomo de oxigênio (O16). Otimizamos a geometria com um $\mathrm{H}^{+}$ligado ao $\mathrm{O} 16$ e posteriormente retiramos $\mathrm{o} \mathrm{H}^{+}$mantendo a geometria. Essa geometria apresenta uma separação de cargas e alternância das ligações simples e duplas em relação a forma neutra. Na figura 6.3 temos uma ilustração da forma zwiteriônica e na tabela 6.3 apresentamos algumas propriedades que foram analisadas. 
Tabela 6.2: Valores de energia $(\mathrm{kcal} / \mathrm{mol})$, momento de dipolo (D), carga atômica (e), comprimento da ligação $(\AA)$ e ângulos diedros $\left(^{\circ}\right)$ para as sondas solvatocrômicas estudadas isoladamente na conformação cis. Geometria otimizada no nível B3LYP/6-311+G** e momento de dipolo obtido com MP2/6-311+G**.

\begin{tabular}{cccc}
\hline \hline Propriedades & cis-MePMBr & cis-MeQMBr & cis-MeAMBr \\
\hline Energia $(\mathrm{kcal} / \mathrm{mol})$ & -3651163.720 & -3747599.510 & -3844029.090 \\
Dipolo (D) & 13.919 & 12.663 & 8.532 \\
Carga (N6) & 0.156 & -0.075 & -0.315 \\
Carga (C8) & -0.374 & -0.192 & -0.129 \\
Carga $(\mathrm{C} 9)$ & -0.018 & -0.110 & -0.070 \\
Carga (O16) & -0.549 & -0.542 & -0.529 \\
Carga(C15) & 0.650 & 0.656 & 0.712 \\
R(C8-C9) & 1.408 & 1.411 & 1.431 \\
R(C15-O16) & 1.223 & 1.222 & 1.219 \\
$\Phi(\mathrm{C} 4-\mathrm{C} 3-\mathrm{C} 8-\mathrm{C} 9)$ & -9.876 & 13.718 & 168.730 \\
$\Phi(\mathrm{C} 8-\mathrm{C} 9-\mathrm{C} 10-\mathrm{C} 12)$ & -14.791 & -170.788 & 178.781 \\
$\Phi(\mathrm{C} 3-\mathrm{C} 8-\mathrm{C} 9-\mathrm{C} 10)$ & -28.539 & 30.533 & -36.439 \\
$\Delta E\left(E_{\text {cis }}-E_{\text {trans }}\right)$ & 6.340 & 6.550 & 8.170 \\
\hline \hline
\end{tabular}




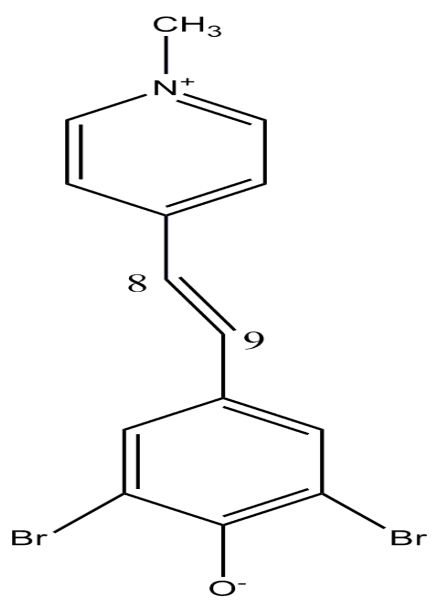

(a) $\mathrm{MePMBr}_{2}$

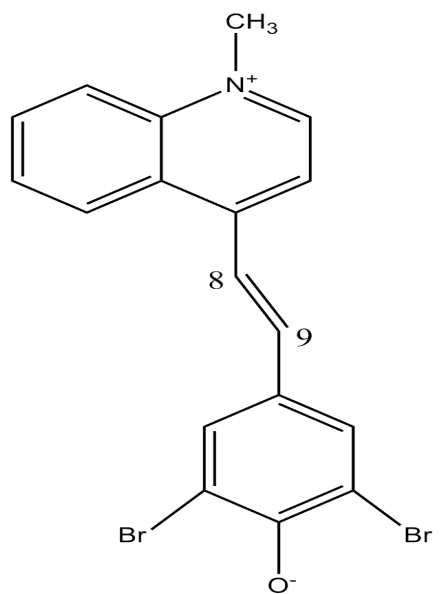

(b) $\mathrm{MeQMBr}_{2}$<smiles></smiles>

(c) $\mathrm{MeAMBr}_{2}$

Figura 6.3: Ilustração das geometrias das sondas estudadas na forma zwiteriônica. Conformação trans. 
Tabela 6.3: Valores de energia(kcal/mol), momento de dipolo (D), carga atômica(e), comprimento da ligação $(\AA)$ e ângulos diedros $\left(^{\circ}\right)$ para as sondas solvatocrômicas na conformação trans estudadas na forma zwiteriônica (ZW). Geometria otimizada no nível B3LYP/6$311+\mathrm{G}^{* *}$ e momento de dipolo obtido com MP2/6-311+G**.

\begin{tabular}{cccc}
\hline \hline Propriedades & ZW-MePMBr & ZW-MeQMBr & ZW-MeAMBr \\
\hline Energia (kcal/mol $)$ & -3651157.940 & -3747593.200 & -3844020.630 \\
Dipolo (D) & 21.456 & 20.013 & 18.213 \\
Carga (N6) & 0.165 & -0.052 & -0.263 \\
Carga (C8) & -0.370 & -0.196 & -0.046 \\
Carga $(\mathrm{C} 9)$ & -0.083 & -0.168 & -0.189 \\
Carga $(\mathrm{O} 16)$ & -0.575 & -0.570 & -0.560 \\
Carga $(\mathrm{C} 15)$ & 0.554 & 0.561 & 0.544 \\
R(C8-C9) & 1.361 & 1.362 & 1.361 \\
R(C15-O16) & 1.331 & 1.332 & 1.333 \\
$\Phi(\mathrm{C} 4-\mathrm{C} 3-\mathrm{C} 8-\mathrm{C} 9)$ & 0.188 & -11.663 & 143.567 \\
$\Phi(\mathrm{C} 8-\mathrm{C} 9-\mathrm{C} 10-\mathrm{C} 12)$ & 0.082 & -3.443 & 171.115 \\
$\Phi(\mathrm{C} 3-\mathrm{C} 8-\mathrm{C} 9-\mathrm{C} 10)$ & 179.984 & 179.424 & 177.376 \\
$\Delta E\left(E_{Z W}-E_{\text {neutra }}\right)$ & 12.120 & 12.860 & 16.610 \\
\hline \hline
\end{tabular}


A forma zwiteriônica foi obtida apenas para o isômero trans, pois como foi verificado anteriormente está é a forma mais estável. Comparando a tabela 6.3 com a tabela 6.1, obtida para a forma neutra, observamos que, isoladamente, a forma neutra é mais estável. Numa análise comparativa, entre as formas neutra e zwiteriônica, das ligações C8-C9 e C15-O16 observa-se que o acréscimo de anéis aromáticos na estrutura $\mathrm{MePMBr}_{2}$ na forma neutra provoca um aumento do caráter quinoidal (aumento no comprimento da ligação C8-C9) porém, para a forma zwiteriônica observa-se poucas mudanças no comprimento das ligações consideradas.

Uma outra possibilidade analisada foi a protonação das moléculas. Na etapa de geração da forma zwiteriônica o próton que é aproximado da molécula é, posteriormente, retirado após a otimização da geometria. Aqui, isso não é feito, e obtemos a geometria com um $\mathrm{H}^{+}$, que em um ambiente aquoso, poderia ser cedido pela água para a sonda. Na figura 6.4 temos uma ilustração dessas geometrias e na tabela 6.4 apresentamos algumas propriedades, lembrando que essa composição só é possível de ser obtida se a molécula estiver embebida num solvente prótico. Aqui estamos apenas levantando essa possibilidade e posteriormente iremos verificar qual a sua contribuição na descrição do espectro eletrônico de absorção das sondas estudadas. 


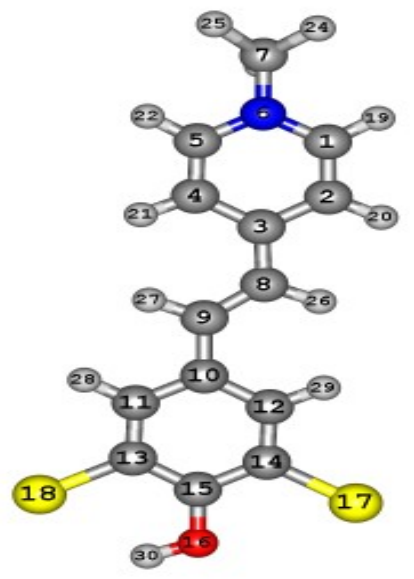

(a) $\mathrm{MePMBr}_{2}$

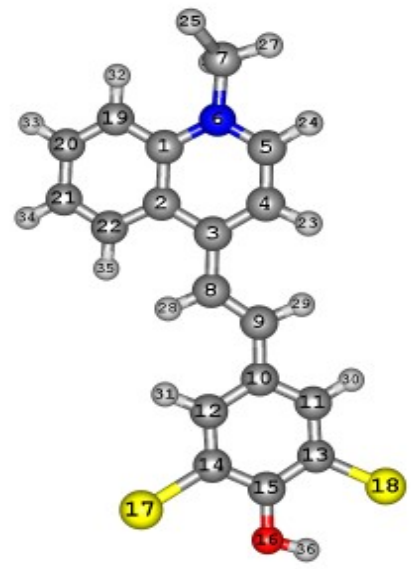

(b) $\mathrm{MeQMBr}_{2}$

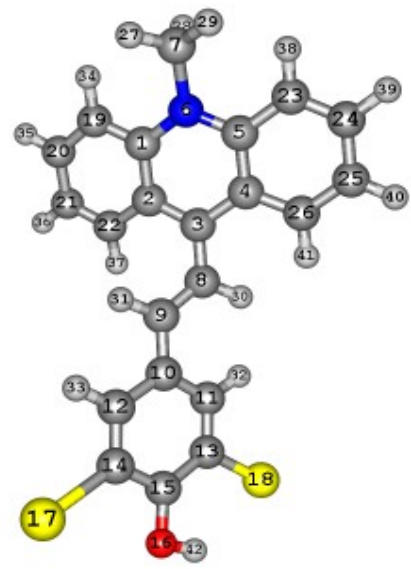

(c) $\mathrm{MeAMBr}_{2}$

Figura 6.4: Ilustração da forma protonada para as sondas. Conformação trans

Tabela 6.4: Valores de energia $(\mathrm{kcal} / \mathrm{mol})$, momento de dipolo (D), carga atômica (e) e comprimento da ligação $(\AA)$ para as sondas na geometria protonada na conformação trans. Geometria otimizada no nível B3LYP/6-311+ $\mathrm{G}^{* *}$ e momento de dipolo obtido com MP2/6$311+\mathrm{G}^{* *}$.

\begin{tabular}{cccc}
\hline \hline Propriedades & $\mathrm{MePMBr}_{2}$ & $\mathrm{MeQMBr}_{2}$ & $\mathrm{MeAMBr}_{2}$ \\
\hline Energia (kcal/mol) & -3651430.53 & -3747865.72 & -3844293.22 \\
Dipolo (D) & 21.754 & 18.786 & 16.300 \\
Carga (N6) & 0.098 & -0.091 & -0.280 \\
Carga (C8) & -0.340 & -0.203 & -0.114 \\
Carga (C9) & -0.007 & -0.093 & -0.098 \\
Carga $(\mathrm{O} 16)$ & -0.534 & -0.540 & -0.543 \\
Carga $(\mathrm{C} 15)$ & 0.434 & 0.446 & 0.425 \\
$\mathrm{R}(\mathrm{C} 8-\mathrm{C} 9)$ & 1.361 & 1.362 & 1.361 \\
$\mathrm{R}(\mathrm{C} 15-\mathrm{O} 16)$ & 1.331 & 1.332 & 1.333 \\
$\Delta E\left(E_{\text {protonada }}-E_{\text {neutra }}\right)$ & -260.47 & -259.66 & -255.98 \\
\hline \hline
\end{tabular}




\subsubsection{Espectro de Absorção}

Na seção anterior descrevemos as possibilidades analisadas para o estado fundamental das sondas estudadas. Agora, apresentaremos os resultados referentes ao estudo de uma propriedade em particular, que é o espectro eletrônico de absorção. Como suporte experimental temos valores para a transição eletrônica das três sondas em 38 solventes [30] (ver gráficos na figura 1.3). Os valores teóricos apresentados aqui foram obtidos com método ab inito TD-DFT |76, 77 tal como implementado no programa Gaussian/03 [56].Experimentalmente, foi observado grandes deslocamentos nas bandas de absorção das três sondas, quando há mudança na polaridade do solvente. A descrição teórica desse comportamento ainda é desafio, visto que estes deslocamentos devem envolver mudanças na geometria do soluto e processos de separação de cargas com a formação do estado zwiteriônico, comportamento característico de moléculas da classe das merocianinas. Resultados téoricos para a energia de transição eletrônica de merocianinas em solventes de baixa polaridade, em geral, não apresentam uma boa descrição para o resultado experimental. Essa informação foi dita anteriormente e levantamos a possibilidade de que um dos motivos para o erro estaria na definição da estrutura para o estado fundamental das sondas. Por conta disso, foram analisadas várias possibilidades e descrita as suas informações estruturais. Outro fator que pode provocar essa má descrição está relacionado com o método utilizado. Por este motivo, nessa seção testaremos alguns métodos para avaliarmos a sua acurácia com relação ao espectro de absorção em merocianinas. Como referência, nós consideramos a merocianina de Brooker (MB) e a sonda $\mathrm{MePMBr}_{2}$ usando diferentes funcionais de densidade mantendo a função base $6-311+\mathrm{G}^{* *}$, ver tabela 6.5. Como referência no nível máximo de cálculo, teremos o resultado obtido com EOMCCSD/6-31G* para a sonda MB, esse resultado não foi obtido para a sonda $\mathrm{MePMBr}_{2}$ por impossibilidades técnicas por a molécula ser muito grande. Para comparação, nós podemos estimar qual o valor da energia de máxima absorção para a molécula isolada (vácuo). Isso foi feito para a 
sonda $\mathrm{MePMBr}_{2}$ pela extrapolação da energia de máxima absorção, $E_{\max }$, usando a regressão linear no gráfico da energia de máxima absorção experimental, $E_{\text {max }}$, versus a escala de polaridade de Reichardt normalizada, $E_{T}^{N}$, figura 6.5. Nessa escala, em vácuo temos que $E_{T}^{N}=-0.11$, o que nos dá um valor de $E_{\text {max }}$ igual a $13884 \mathrm{~cm}^{-1}(720$ nm) para a sonda $\mathrm{MePMBr}_{2}$.

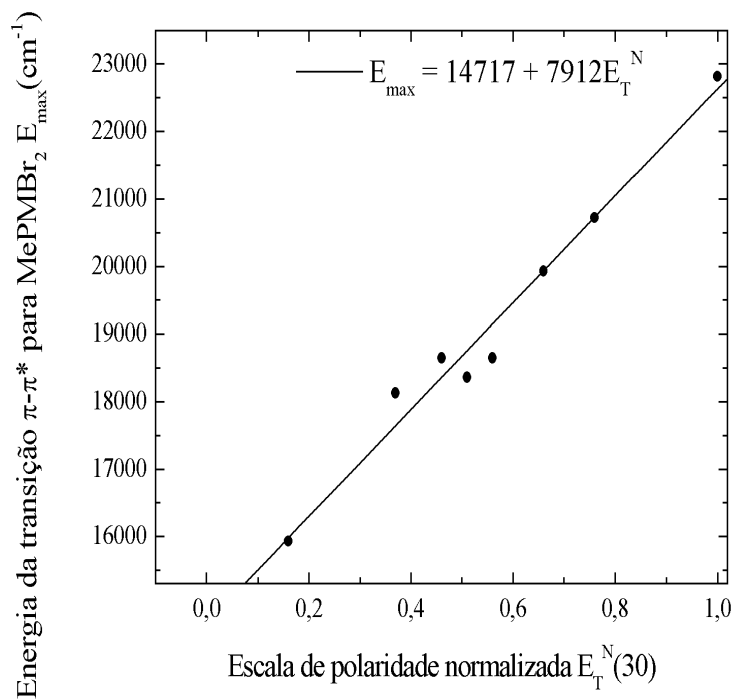

Figura 6.5: Energia de máxima absorção experimental, $E_{\text {max }}$, para a sonda $\mathrm{MePMBr}_{2}$ versus a escala de polaridade de Reichardt normalizada, $E_{T}^{N}$ [30]. Os solventes considerados foram: 1,4 Dioxano(1); (2) ; Acetonitrila(3); Cicloexano(4); 2-Metil-1-butanol(5); 2-Metoxietanol(6); Metanol(7) e Água(8).

As geometrias consideradas nesse cálculo foram obtidas isoladamente, MB (obtida com PBEPBE/6-31G*) e $\mathrm{MePMBr}_{2}$ (obtida com B3LYP/6-311+G**). Com relação a estrutura, MB possui a ligação C8-C9 igual a $1.408 \AA$ (valor da ligação C8-C9 obtida para a $\mathrm{MePMBr}_{2}$ foi $1.400 \AA$ ), e para a ligação C15-O16 o valor de $1.251 \AA$ (valor da ligação C15-O16 obtida para a $\mathrm{MePMBr}_{2}$ foi $1.223 \AA$ ). 
Tabela 6.5: Valores para energia da transição $\pi-\pi^{*}\left(\mathrm{~cm}^{-1}\right)$ e comprimento de onda (nm) para as sondas $\mathrm{MB}$ e $\mathrm{MePMBr}_{2}$ obtidos utilizando diferentes métodos de cálculos quânticos.

\begin{tabular}{ccc}
\hline \hline Método de cálculo & MB & $\mathrm{MePMBr}_{2}$ \\
\hline B3LYP & $20285(493)$ & $21510(465)$ \\
BHandHLYP & $23301(429)$ & $22809(438)$ \\
CAM-B3LYP & $22696(440)$ & $22138(452)$ \\
WB97XD & $22646(442)$ & $22091(453)$ \\
LC-WPBE & $22978(435)$ & $22389(447)$ \\
EOM-CCSD & $24197(413)$ & - \\
Valor estimado (gás) & - & $13884(720)$ \\
Valor em clorofórmio |30 & - & $16129(620)$ \\
Valor em 1,4 dioxano $|30|$ & - & $16000(625)$ \\
\hline \hline
\end{tabular}


Observa-se na tabela 6.5 que todos os métodos erram na descrição para o valor absoluto da energia da transição eletrônica. Decidimos então usar os funcionais B3LYP e em alguns casos, CAM-B3LYP, previamente utilizados em outros trabalhos para estudar efeitos de solventes em espectros eletrônicos de absorção [3, 119,120$]$.

Na tabela 6.6 apresentamos as energias de transição e os comprimentos de onda das transições em vácuo usando o funcional B3LYP para todas as geometrias geradas para as sondas (formas trans, cis, trans-ZW e trans-protonada). Observa-se que a forma cis, comparativamente a forma trans, desloca a transição para menor energia por cerca de $-1600 \mathrm{~cm}^{-1}(\approx 40 \mathrm{~nm})$. Essa diferença não é grande quando comparado à largura da $1^{\circ}$ banda do espectro, que tem largura de cerca de $150 \mathrm{~nm}$. A forma trans-ZW também desloca a transição para menor energia por cerca de $-2500 \mathrm{~cm}^{-1}$ para as sondas $\mathrm{MePMBr}_{2}$ e $\mathrm{MeQMBr}_{2}$, e $-4500 \mathrm{~cm}^{-1}$ para a sonda $\mathrm{MeAMBr}_{2}$. Já a forma protonada desloca a transição para maiores energias por cerca de $200 \mathrm{~cm}^{-1}$ para a sonda $\mathrm{MePMBr}_{2}, 800 \mathrm{~cm}^{-1}$ para a sonda $\mathrm{MeQMBr}_{2}$ e para menores energia por cerca de $-800 \mathrm{~cm}^{-1}$ para a sonda $\mathrm{MeAMBr}_{2}$.

Tabela 6.6: Valores para energia da transição $\pi-\pi^{*}\left(\mathrm{~cm}^{-1}\right)$ para as sondas estudadas obtidos utilizando as diferentes estruturas otimizadas para o estado fundamental da molécula. Entre parênteses apresentamos o valor para o comprimento de onda de máxima absorção (nm).

\begin{tabular}{cccc}
\hline \hline Geometria & $\mathrm{MePMBr}_{2}$ & $\mathrm{MeQMBr}_{2}$ & $\mathrm{MeAMBr}_{2}$ \\
\hline Trans & $21645(462)$ & $19802(505)$ & $19305(518)$ \\
Cis & $19960(501)$ & $18282(547)$ & $17606(568)$ \\
ZW & $19231(520)$ & $17241(580)$ & $15083(663)$ \\
Protonada & $21834(458)$ & $20661(484)$ & $18484(541)$ \\
\hline \hline
\end{tabular}




\subsection{Molécula em solução}

Utilizamos dois modelos para o solvente como descrito no capítulo 3: o modelo contínuo polarizável, PCM, e o modelo discreto. No PCM o meio é tratado de forma homogênea incluindo na equação de Schrödinger a interação elétrons e prótons do soluto com um meio dielétrico polarizável. A utilização do PCM pode ser feita apenas nas propriedades eletrônicas, mantendo a geometria rígida de vácuo; nos cálculos em que otimizam a geometria, permitindo uma relaxação na geometria do soluto devido ao solvente; e nos cálculos das propriedades eletrônicas com a geometria relaxada em solução. Desta forma, comparando esses resultados que incluem o solvente apenas na estrutura eletrônica e/ou na geometria, podemos perceber a contribuição separadamente de cada termo. Faremos isso em seguida. No modelo discreto, as moléculas do solvente são posicionadas ao redor do soluto através de simulações computacionais. Aqui, utilizamos o método Monte Carlo com moléculas rígidas para gerar as configurações do sistema soluto-solvente e posteriormente realizar os cálculos de estrutura eletrônica. As geometrias do soluto utilizadas nas simulações foram relaxadas em solução utilizando o modelo contínuo PCM.

\subsubsection{Efeito do solvente no espectro eletrônico de absorção: modelo contínuo}

- Forma neutra trans

Os valores para as energias de transição obtidas através de cálculos teóricos estão apresentados na tabela 6.7, lá também apresentamos os respectivos valores experimentais. Nós realizamos cálculos com TD-DFT usando o funcional B3LYP considerando a molécula em solução de 1,4 dioxano (baixa polaridade) e em meio aquoso (alta polaridade) através do modelo contínuo polarizável, PCM. As geometrias utilizadas nesses cálculos foram otimizadas em vácuo. Nosso interesse é analisar o efeito do solvente 
apenas na estrutura eletrônica. Esses resultados teóricos estão comparados aos deslocamentos sofridos por cada molécula quando há uma mudança na polaridade do meio em que essa está solvatada, nesse caso, devido a mudança de 1,4 dioxano (baixa polaridade) para água(alta polaridade). Esses resultados estão apresentados na tabela 6.7 A transição mais intensa, em todas as três sondas, foi caracterizada como sendo do tipo $\pi-\pi^{*}$, ver figura 6.6 com a ilustração dos orbitais (HOMO $\Longrightarrow$ LUMO) envolvidos na excitação.

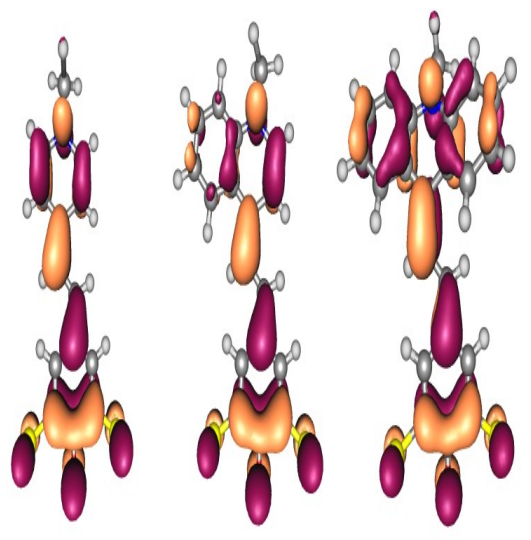

(a) $\operatorname{HOMO}(\pi)$
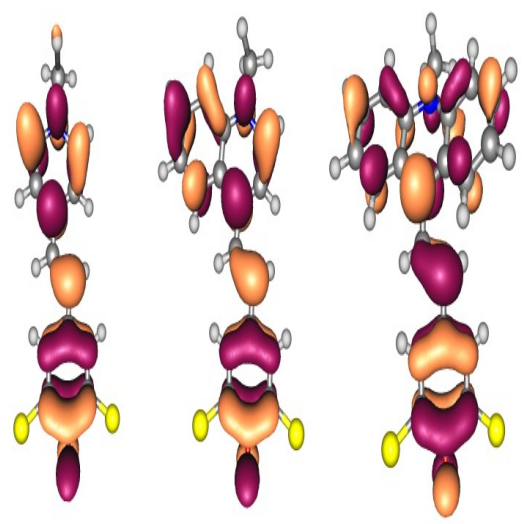

(b) $\operatorname{LUMO}\left(\pi^{*}\right)$

Figura 6.6: Ilustração dos orbitais moleculares para as sondas $\mathrm{MePMBr}_{2}, \mathrm{MeQMBr}_{2}$ e $\mathrm{MeAMBr}_{2}$, respectivamente, obtidos com TD-B3LYP $/ 6-311+\mathrm{G}^{* *}$. Orbitais para as moléculas isoladas.

Observa-se na tabela 6.7 que o resultado experimental apresenta um deslocamento para maiores energias (ou menores comprimentos de onda) com o aumento da polaridade (1,4 dioxano $\rightarrow$ água). Esse comportamento é conhecido na literatura como deslocamento hipsocrômico (ou deslocamento para o azul) caracterizando um solvatocromismo negativo. Por outro lado, o nosso resultado teórico obtido com TDB3LYP $/ 6-311+\mathrm{G}^{* *}$ apresenta um comportamento contrário ao experimental, com o aumento da polaridade ocorre um deslocamento para menores energias (ou maiores comprimentos de onda), esse deslocamento é conhecido como batocrômico (ou deslocamento para o vermelho) e caracteriza um solvatocromismo positivo. Portanto con- 
cluimos que é necessário considerar outros efeitos adicionais aqueles obtidos considerando o solvente apenas na estrutura eletrônica mantendo a geometria rígida de vácuo. Porém vale ressaltar que os valores para $\lambda_{\max }$ calculados para a excitação em água, $478 \mathrm{~nm}$ para $\mathrm{MePMBr}_{2}, 522 \mathrm{~nm}$ para $\mathrm{MeQMBr}_{2}$ e $570 \mathrm{~nm}$ para $\mathrm{MeAMBr}_{2}$, estão próximos aos valores experimentais 438, 480 e $560 \mathrm{~nm}$, respectivamente. Enquanto que os valores calculados para o solvente 1,4 dioxano estão subestimando os valores experimentais por cerca de $150 \mathrm{~nm}$.

Tabela 6.7: Valores para energia da transição $\pi-\pi^{*}, \mathrm{E}\left(\mathrm{cm}^{-1}\right)$, e comprimento de onda, $\lambda_{\max }$ $(\mathrm{nm})$, para as sondas estudadas na conformação trans. Valores teóricos obtidos com TD-DFT usando o funcional B3LYP e as funções base 6-311+G**.

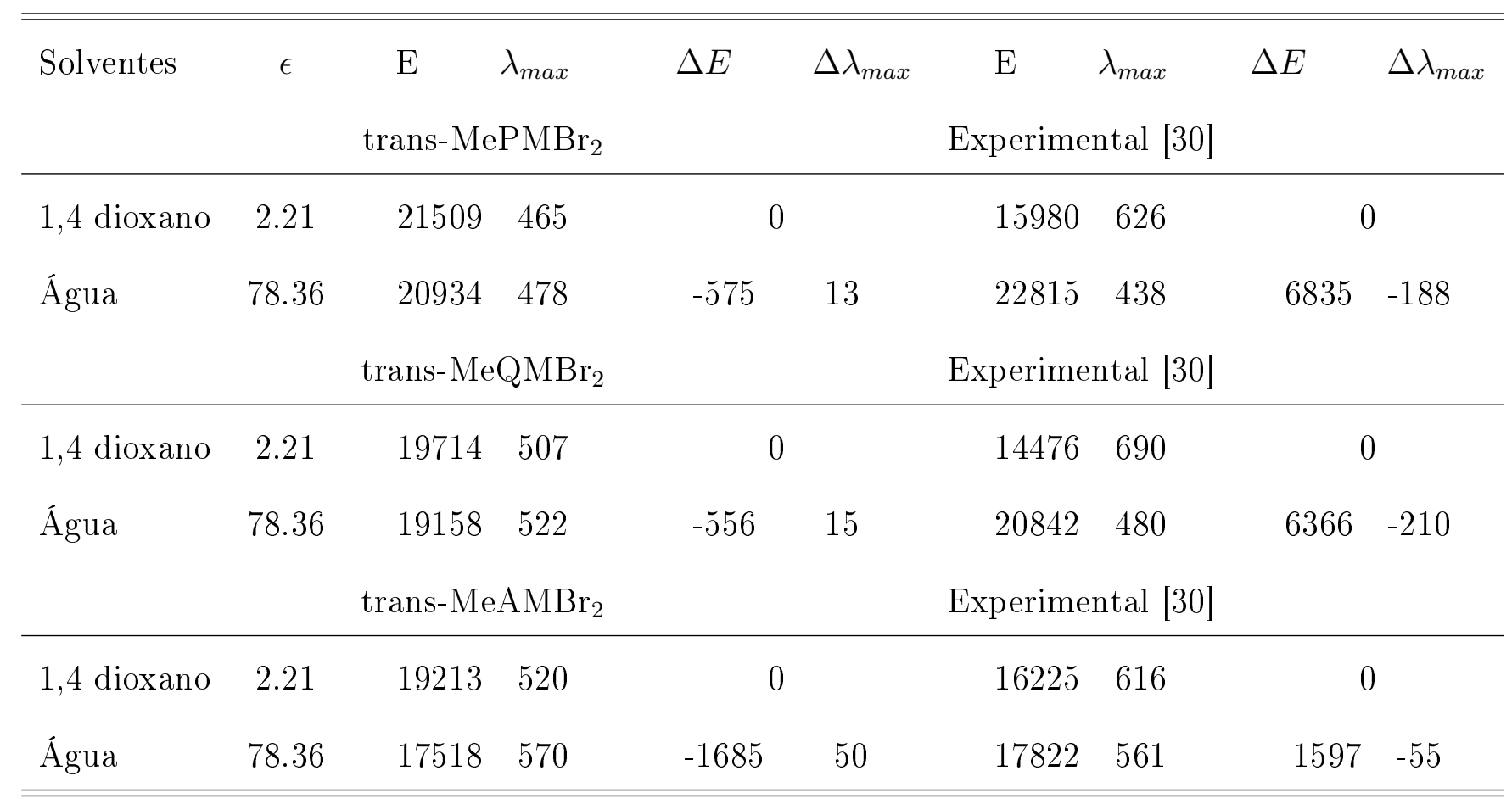

- Forma neutra cis 
Nós realizamos o mesmo estudo do espectro de absorção nas geometrias das sondas na forma cis. Na tabela 6.8 são apresentados os nossos resultados, onde se observa o mesmo comportamento anterior, o método TD-DFT com o funcional B3LYP continua não descrevendo de maneira correta o solvatocromismo sofrido pelas sondas. Com relação aos valores das transições há uma mudança quando comparamos com os valores obtidos para a forma trans (ver tabela 6.7), por cerca de $-1500 \mathrm{~cm}^{-1}$ para ambas soluções. Porém quando analisamos os deslocamentos sofrido na banda, $\Delta E\left(\mathrm{~cm}^{-1}\right)$, a mudança é pequena, cerca de $1 \mathrm{~nm}$. Em outros trabalhos [30], cálculos teóricos foram realizados e foi observado que o comprimento de onda de máxima absorção da forma cis é praticamente o mesmo apresentado para a forma trans, indicando que através de espectroscopia em UV-Vis não é possível detectar qual das formas é responsável pela absorção. 
Tabela 6.8: Valores para energia da transição $\pi-\pi^{*}, \mathrm{E}\left(\mathrm{cm}^{-1}\right)$, e comprimento de onda, $\lambda_{\max }$ $(\mathrm{nm})$, para as sondas estudadas na conformação cis. Valores teóricos obtidos com TD-DFT usando o funcional B3LYP e as funções base $6-311+\mathrm{G}^{* *}$.

\begin{tabular}{|c|c|c|c|c|c|c|c|c|c|}
\hline Solventes & $\epsilon$ & $\begin{array}{l}\mathrm{E} \\
\text { cis-MeF }\end{array}$ & $\begin{array}{l}\lambda_{\max } \\
\mathrm{MBr}_{2}\end{array}$ & $\Delta E$ & $\Delta \lambda_{\max }$ & $\begin{array}{c}\text { E } \\
\text { Experime }\end{array}$ & $\begin{array}{l}\lambda_{\max } \\
\text { ntal }|30|\end{array}$ & $\Delta E$ & $\Delta \lambda_{\max }$ \\
\hline 1,4 dioxano & 2.21 & 19880 & 503 & 0 & & 15980 & 626 & 0 & 0 \\
\hline Água & 78.36 & $\begin{array}{r}19454 \\
\text { cis-MeG }\end{array}$ & $\begin{array}{c}514 \\
\mathrm{MBr}_{2}\end{array}$ & -426 & 11 & $\begin{array}{r}22815 \\
\text { Experime }\end{array}$ & 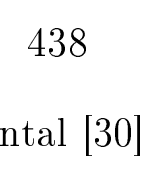 & 6835 & -188 \\
\hline 1,4 dioxano & 2.21 & 18229 & 548 & 0 & & 14476 & 690 & 0 & 0 \\
\hline Água & 78.36 & $\begin{array}{r}17718 \\
\text { cis-MeA }\end{array}$ & $\begin{array}{c}564 \\
\mathrm{MBr}_{2}\end{array}$ & -511 & 16 & $\begin{array}{r}20842 \\
\text { Experime }\end{array}$ & $\begin{array}{c}480 \\
\text { ntal } \mid 30\end{array}$ & 6366 & -210 \\
\hline 1,4 dioxano & 2.21 & 17462 & 573 & 0 & & 16225 & 616 & 0 & 0 \\
\hline Água & 78.36 & 16080 & 622 & -1382 & 49 & 17822 & 561 & 1597 & -55 \\
\hline
\end{tabular}


- Forma protonada trans

Outra possibilidade estudada foi a forma protonada em que a molécula receberia um hidrogênio $\left(\mathrm{H}^{+}\right)$doado pelo solvente. Até o momento o solvente está sendo tratado apenas através do modelo contínuo, em que interações específicas solutosolvente (ligações de hidrogênio, por exemplo) não são levadas em conta. Portanto, o nosso interesse aqui é apenas verificar o efeito da protonação no espectro de absorção das sondas estudadas. Na tabela 6.9 apresentamos os resultados obtidos usando TDB3LYP $/ 6-311+\mathrm{G}^{* *}$. As formas protonadas apresentam excitação eletrônica na região de maior energia, comparativamente à forma neutra, cerca de $3500 \mathrm{~cm}^{-1}, 3100 \mathrm{~cm}^{-1}$ e $2100 \mathrm{~cm}^{-1}$ para $\mathrm{MePMBr}_{2}, \mathrm{MePMBr}_{2}$ e $\mathrm{MePMBr}_{2}$, respectivamente, em água. Não temos disponíveis os valores experimentais para essas sondas protonadas em água, porém o deslocamento experimental para a sonda MB devido a protonação é de cerca de $3300 \mathrm{~cm}^{-1}$ em água [33], portanto compatível com os valores que calculamos. 
Tabela 6.9: Valores para energia da transição $\pi-\pi^{*}, \mathrm{E}\left(\mathrm{cm}^{-1}\right)$, e comprimento de onda, $\lambda_{\max }$ $(\mathrm{nm})$, para as sondas estudadas na forma protonada. Valores teóricos obtidos com TD-DFT usando o funcional B3LYP e as funções base $6-311+\mathrm{G}^{* *}$.

\begin{tabular}{lccccc}
\hline \hline Solventes & $\epsilon$ & $\mathrm{E}$ & $\lambda_{\text {max }}$ & $\Delta E$ & $\Delta \lambda_{\text {max }}$ \\
& \multicolumn{5}{c}{$\mathrm{MePMBr}_{2} \mathrm{H}^{+}$} \\
1,4 dioxano & 2.21 & 22573 & 443 & \multicolumn{2}{c}{0} \\
Água & 78.36 & 24464 & 409 & 1891 & -34 \\
& & $\mathrm{MeQMBr}_{2} \mathrm{H}^{+}$ & & \\
\hline 1,4 dioxano & 2.21 & 20833 & 480 & & 0 \\
Água & 78.36 & 22275 & 449 & 1442 & -31 \\
& & $\mathrm{MeAMBr}_{2} \mathrm{H}^{+}$ & & \\
\hline 1,4 dioxano & 2.21 & 18726 & 534 & \multicolumn{2}{c}{0} \\
Água & 78.36 & 19632 & 509 & 906 & -25 \\
\hline \hline
\end{tabular}


- Forma trans zwiteriônica

A forma zwiteriônica das sondas foi obtida aproximando um próton e gerando uma geometria com separação de cargas. Obter informação sobre esse estado é importante, pois a composição do estado fundamental das merocianinas pode ser descrito por um híbrido de ressonância entre as formas neutra e zwiteriônica. Na tabela 6.10 são apresentados os valores para a energia de transição e também o deslocamento sofrido pela banda devido a mudança na polaridade do meio, os resultados foram obtidos com TD-DFT para ambos solventes. Observa-se na tabela 6.10 que a mudança na polaridade do solvente está provocando um deslocamento para maiores energias, assim como acontece no resultado experimental, porém vale ressaltar que assumimos a forma zwiteriônica para o estado fundamental das sondas mesmo em solvente de baixa polaridade e apolar (na tabela os valores estão referidos a 1,4 dioxano), o que de fato não acontece, como vimos antes, o estado fundamental das sondas estudadas se encontra na forma neutra (quinoidal). Como vimos na tabela 6.3 a forma zwiteriônica apresenta uma aumento de energia, quando comparado com a forma neutra, em cerca de 12, 13 e $17 \mathrm{kcal} / \mathrm{mol}$, para as sondas $\mathrm{MePMBr}_{2}, \mathrm{MeQMBr}_{2}$ e $\mathrm{MeAMBr}_{2}$, respectivamente. Apresenta também um aumento no momento de dipolo em 7.6, 7.3 e $9.7 \mathrm{D}$ para as sondas $\mathrm{MePMBr}_{2}, \mathrm{MeQMBr}_{2}$ e $\mathrm{MeAMBr}_{2}$, respectivamente. Dessa forma, a estabilização da forma ZW num solvente polar é factível, pois a interação soluto-solvente poderá liberar a energia necessária para que ocorra a mudança neutra $\rightarrow Z W$, uma vez que a forma ZW possui um maior momento de dipolo. Porém não conseguimos encontrar justificativas para a estabilização da forma ZW em solventes apolares. Pois qual seria a interação soluto-solvente predominante que liberaria a energia necessária para estabilizar a forma ZW? Aqui deixaremos essa questão que será retomada ao final de todos os nossos cálculos e análises. 
Tabela 6.10: Valores para energia da transição $\pi-\pi^{*}, \mathrm{E}\left(\mathrm{cm}^{-1}\right)$, e comprimento de onda, $\lambda_{\max }(\mathrm{nm})$, para as sondas estudadas na forma zwiteriônica. Valores teóricos obtidos com TD-DFT usando o funcional B3LYP e as funções base $6-311+\mathrm{G}^{* *}$.

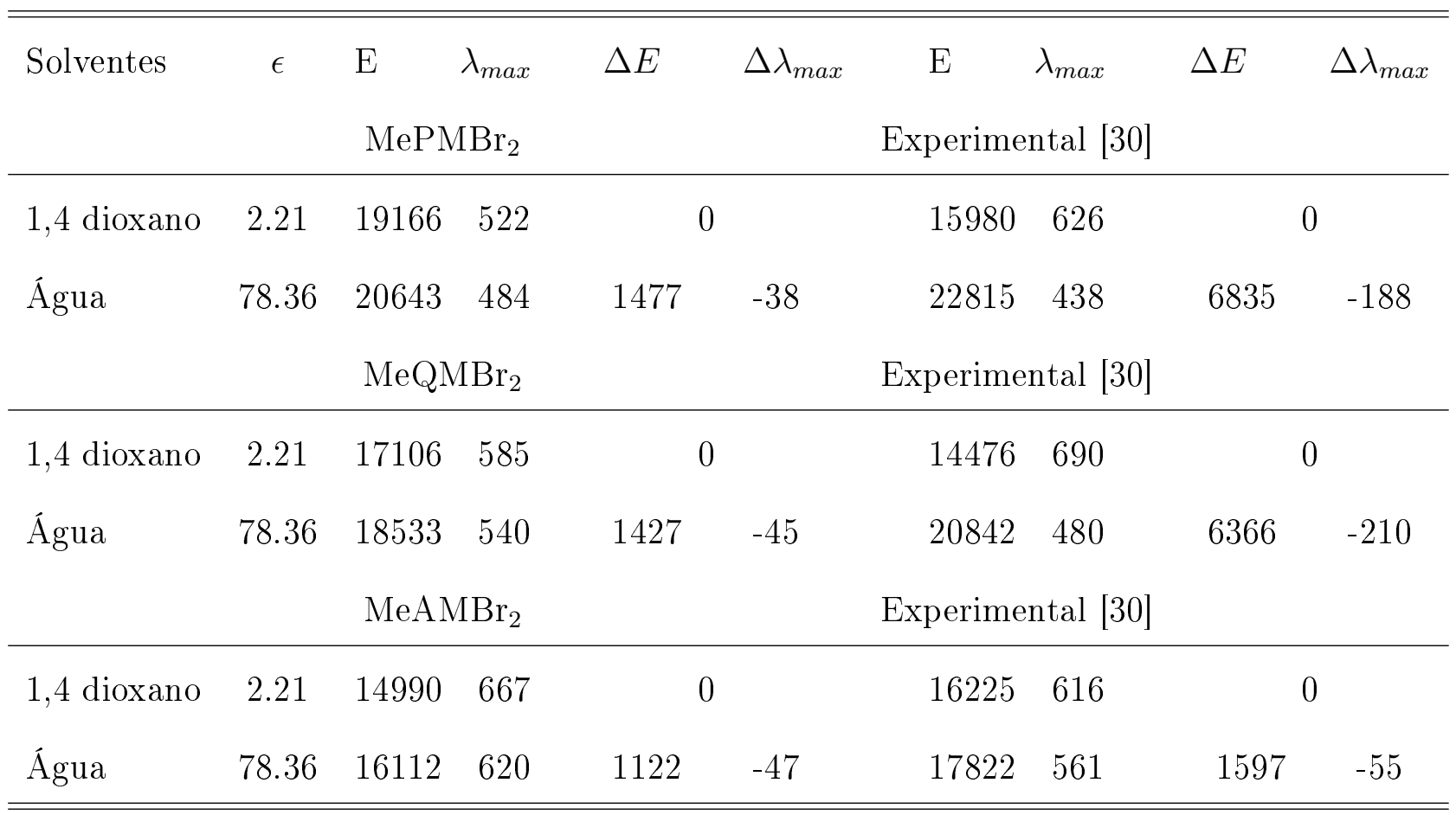




\subsubsection{Efeito solvente na geometria e no espectro eletrônico: modelo contínuo}

Até o momento as geometrias do estado fundamental consideradas foram obtidas com as moléculas em vácuo. Como vimos, a forma neutra (quinoidal) foi obtida através de cálculos quânticos e a forma zwiteriônica foi obtida através da aproximação de um próton $\mathrm{H}^{+}$da molécula de interesse, o que mimetiza uma interação específica solutosolvente. Nesta seção discutimos o efeito dessa interação soluto-solvente na geometria e no espectro de absorção. O solvente foi considerado através do modelo contínuo polarizável PCM. Para obter o efeito na estrutura nós otimizamos as geometrias das sondas em quatro solventes, foram eles: 1,4 dioxano, DMSO, metanol e água. Na figura 6.7. nós analisamos as ligações C8-C9 e C15-O16. Para obtenção das geometrias em PCM nós realizamos cálculos com o funcional B3LYP e as funções base $6-311+\mathrm{G}^{* *}$.

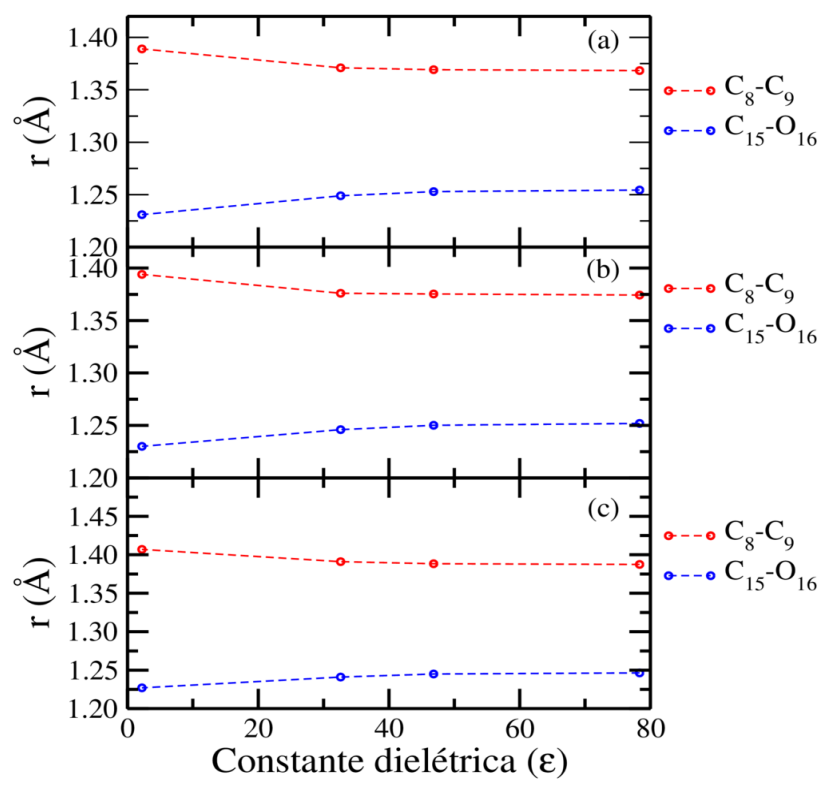

Figura 6.7: Comprimento das ligações C8-C9 e C15-O16, obtidos com B3LYP/6-311+G**, nos solventes: 1,4 dioxano $(\epsilon=2.21)$, metanol $(\epsilon=32.61)$, DMSO $(\epsilon=46.83)$ e água $(\epsilon=78.36)$. Legenda do gráfico: (a) $\mathrm{MePMBr}_{2}$, (b) $\mathrm{MeQMBr}_{2}$ e (c) $\mathrm{MeAMBr}_{2}$.

Observa-se na figura 6.7 que na medida em que aumentamos a constante dielétrica do solvente ocorre uma diminuição no comprimento da ligação C8-C9 (aumenta o 
caráter de uma ligação dupla), por exemplo, para a sonda $\mathrm{MePMBr}_{2}$ temos que o comprimento da ligação é de $1.389 \AA$ para a sonda em 1,4 dioxano, e em água o comprimento dessa ligação é reduzida para $1.368 \AA$. Por outro lado ocorre um aumento na ligação C15-O16 (aumenta o caráter de uma ligação simples), fazendo com que aumente o caráter zwiteriônico a medida que a constante dielétrica do solvente aumenta. Por exemplo, o comprimento dessa ligação é de $1.231 \AA$ para sonda $\mathrm{MePMBr}_{2}$ em 1,4 dioxano, e esse comprimento é aumentado em água para $1.254 \AA$. Com relação ao momento de dipolo, figura 6.8, ocorre um aumento com o acréscimo da constante dielétrica, por exemplo, para a sonda $\mathrm{MePMBr}_{2}$ o seu momento de dipolo em 1,4 dioxano é 17.42 D e em água sofre um aumento para $38.34 \mathrm{D}$, indicando que as sondas são altamente polarizáveis com o meio. Os cálculos para o momento de dipolo foram realizados com o método MP2 e o conjunto de funções base 6-311 $+\mathrm{G}^{* *}$. Observa-se também que o modelo contínuo é pouco sensível para mudanças entre os solventes metanol, DMSO e água, ou seja, para constantes dielétricas altas.

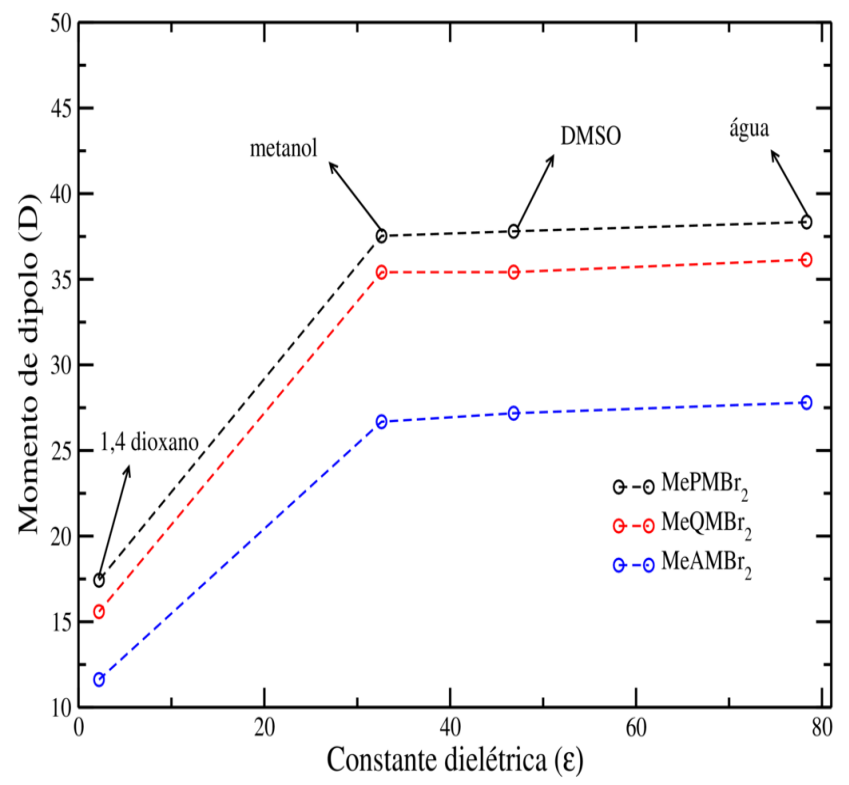

Figura 6.8: Momento de dipolo para as sondas nos diferentes solventes, obtido com MP2/6$311+\mathrm{G}^{*}$. Os solventes são: 1,4 dioxano $(\epsilon=2.21)$, metanol $(\epsilon=32.61)$, DMSO $(\epsilon=46.83)$ e água $(\epsilon=78.36)$. 
Na tabela 6.11 nós apresentamos uma análise para o comportamento da transição eletrônica quando há mudança na geometria. Nesta tabela, a geometria foi relaxada no respectivo solvente considerando o PCM e em seguida foi realizado o cálculo de espectro nessa geometria modificada devido a presença do solvente, porém, para o cálculo do valor da transição eletrônica foi considerado a molécula em vácuo. 
Tabela 6.11: Efeito do solvente apenas na geometria para o espectro de absorção. Valores para energia da transição $\pi-\pi^{*}, \mathrm{E}\left(\mathrm{cm}^{-1}\right)$, e comprimento de onda, $\lambda_{\max }(\mathrm{nm})$ para as sondas estudadas na conformação trans. Valores teóricos obtidos com TD-DFT usando dois funcionais distintos, B3LYP e [CAM-B3LYP], e as funções base 6-311+ $\mathrm{G}^{* *}$. Geometria relaxada no solvente e espectro calculado em vácuo.

\begin{tabular}{lccccc}
\hline \hline Solventes & $\epsilon$ & $\mathrm{E}$ & $\Delta E$ & $\lambda_{\max }$ & $\Delta \lambda_{\max }$ \\
& \multicolumn{5}{c}{$\mathrm{MePMBr}_{2}$} \\
\hline 1,4 dioxano & 2.21 & $21053[21714]$ & 0 & $475[460]$ & 0 \\
Metanol & 32.61 & $19881[20725]$ & $-1172[-980]$ & $503[482]$ & $28[22]$ \\
DMSO & 46.83 & $19881[20708]$ & $-1172[-1006]$ & $503[483]$ & $28[23]$ \\
Água & 78.36 & $19763[20643]$ & $-1290[-1071]$ & $506[484]$ & $31[24]$ \\
& & $\mathrm{MeQMBr} 2$ & & & \\
\hline 1,4 dioxano & 2.21 & $19305[20297]$ & 0 & $518[493]$ & 0 \\
Metanol & 32.61 & $18282[19361]$ & $-1023[-936]$ & $547[516]$ & $29[23]$ \\
DMSO & 46.83 & $18282[19354]$ & $-1023[-943]$ & $547[517]$ & {$[23]$} \\
Água & 78.36 & $18182[19271]$ & $-1123[-1026]$ & $550[518]$ & $32[25]$ \\
\hline Água & 78.36 & $17986[19014]$ & $-1025[-1354]$ & $556[526]$ & $30[35]$ \\
\hline 1,4 dioxano & 2.21 & $19011[20368]$ & 0 & $526[491]$ & 0 \\
Metanol & 32.61 & $18083[19120]$ & $-928[-1248]$ & $553[523]$ & $27[32]$ \\
DMSO & 46.83 & $18018[19065]$ & $-993[-1303]$ & $555[524]$ & $29[33]$ \\
& & MeAMBr & & & \\
\hline
\end{tabular}


De acordo com a tabela 6.11 observamos que o efeito do solvente na geometria provoca um deslocamento na transição eletrônica para menores energias, cerca de - 1200 $\mathrm{cm}^{-1} \mathrm{MePMBr}_{2},-1000 \mathrm{~cm}^{-1} \mathrm{MeQMBr}_{2} \mathrm{e}-1300 \mathrm{~cm}^{-1} \mathrm{MeAMBr}_{2}$. Esse comportamento é observado nos dois funcionais utilizados. Agora, incluindo o efeito do solvente na geometria e no espectro eletrônico nós obtivemos os resultados que estão apresentados na tabela 6.12 .

Na tabela 6.12 nós podemos observar que incluindo os efeitos do solvente nas duas propriedades, na geometria e no espectro, o deslocamento da transição eletrônica apresenta um comportamento distinto do observado na tabela 6.11. Resuminadamente temos que para a sonda $\mathrm{MePMBr}_{2}$, o deslocamento para maiores energias na transição eletrônica é cerca de $800 \mathrm{~cm}^{-1}\left[2000 \mathrm{~cm}^{-1}\right]$, para a sonda $\mathrm{MeQMBr}_{2}$, o deslocamento é cerca de $600 \mathrm{~cm}^{-1}\left[1400 \mathrm{~cm}^{-1}\right]$. A sonda $\mathrm{MeAMBr}_{2}$ apresenta um comportamento diferente do observado para as demais, observamos um deslocamento para menores energias na transição eletrônica, esse valor é cerca de $-500 \mathrm{~cm}^{-1}\left[-700 \mathrm{~cm}^{-1}\right]$. 
Tabela 6.12: Efeito do solvente na geometria e no espectro de absorção. Valores para energia da transição $\pi-\pi^{*}, \mathrm{E}\left(\mathrm{cm}^{-1}\right)$, e comprimento de onda, $\lambda_{\max }(\mathrm{nm})$ para as sondas estudadas na conformação trans. Valores teóricos obtidos com TD-DFT usando dois funcionais distintos, B3LYP e [CAM-B3LYP], e as funções base $6-311+\mathrm{G}^{* *}$. Geometria relaxada no solvente e espectro calculado no respectivo solvente.

\begin{tabular}{|c|c|c|c|c|c|}
\hline Solventes & $\epsilon$ & $\begin{array}{c}\mathrm{E} \\
\mathrm{MePMBr}_{2}\end{array}$ & $\Delta E$ & $\lambda_{\max }$ & $\Delta \lambda_{\max }$ \\
\hline 1,4 dioxano & 2.21 & $19724[20080]$ & 0 & $507[498]$ & 0 \\
\hline Metanol & 32.61 & $20492[22124]$ & $768[2044]$ & $488[452]$ & $-19[-46]$ \\
\hline DMSO & 46.83 & $20325[22026]$ & $601[1946]$ & $492[454]$ & $-15[-44]$ \\
\hline \multirow[t]{2}{*}{ Água } & 78.36 & $20576[22321]$ & $852[2241]$ & 486 [448] & $-21[-50]$ \\
\hline & & $\mathrm{MeQMBr}_{2}$ & & & \\
\hline 1,4 dioxano & 2.21 & $18116[18726]$ & 0 & $552[534]$ & 0 \\
\hline Metanol & 32.61 & $18657[20040]$ & $541[1314]$ & 536 [499] & $-16[-35]$ \\
\hline DMSO & 46.83 & $18484[19881]$ & $368[1155]$ & $541[503]$ & $-11[-31]$ \\
\hline \multirow[t]{2}{*}{ Água } & 78.36 & $18692[20161]$ & $576[1435]$ & $535[496]$ & $-17[-38]$ \\
\hline & & $\mathrm{MeAMBr}_{2}$ & & & \\
\hline 1,4 dioxano & 2.21 & $17544[18587]$ & 0 & $570[538]$ & 0 \\
\hline Metanol & 32.61 & $17391[18149]$ & $-153[-438]$ & $575[551]$ & $5[13]$ \\
\hline DMSO & 46.83 & $17065[17953]$ & $-479[-634]$ & $586[557]$ & $16[19]$ \\
\hline Água & 78.36 & $17301[18149]$ & $-243[-438]$ & $578[551]$ & $8[13]$ \\
\hline
\end{tabular}




\subsubsection{Efeito do solvente no espectro eletrônico de absorção: modelo discreto}

Para considerar as moléculas do solvente de maneira explícita, nós realizamos a solvatação das sondas estudadas nos 4 solventes distintos. Os solventes estudados foram: 1,4 dioxano, dimetilsulfóxido(DMSO), metanol e água. As configurações das sondas solvatadas nos diferentes solventes foram geradas através de simulações computacionais de líquidos moleculares, usando o método Monte Carlo Metropolis. O nosso estudo foi feito considerando as moléculas rígidas, onde as geometrias das sondas foram obtidas realizando cálculos quânticos no nível B3LYP/6-311+G**, utilizando modelo contínuo PCM para cada solvente.

O ensemble utilizado nas simulações foi o NPT, em que são mantidos fixos o número de partículas, a pressão e a temperatura. Para descrever a interação intermolecular entre o soluto e o solvente usamos o potencial Lennard-Jones 101 adicionado à um termo Coulombiano, o potencial é dado pela equação 3.34 .

Como as sondas estudadas nesse trabalho não estão parametrizadas, nós obtivemos os parâmetros $\epsilon$ e $\sigma$ para o potencial Lennard-Jones por semelhança com grupos funcionais que possuem parametrização. Isso foi feito usando o campo de força OPLSAA [104. As cargas do termo de Coulomb foram obtidas considerando a polarização do soluto devido a presença do solvente, realizando-se cálculos quânticos no nível MP2/6-311 $+\mathrm{G}^{* *}$ utilizando o ajuste do potencial eletrostático com o procedimento CHELPG considerando a molécula embebida no solvente através do modelo contínuo polarizável (PCM). Esse procedimento vem sendo usado com sucesso na descrição da polarização de solutos $3,120,122$. Portanto, para cada simulação que será descrita a seguir a geometria do estado fundamental e o conjunto de cargas do soluto que são considerados nas simulações foram obtidos na presença do respectivo solvente através do PCM. 
- Simulações

Como mencionado, o estudo das sondas foi realizado em 4 solventes distinto. Na figura 6.9 ilustramos a estrutura dos solventes. Foi escolhido um solvente apolar e aprótico, 1,4 dioxano (densidade $=1.03 \mathrm{~g} / \mathrm{cm}^{3}$, dipolo=0.00 D), um solvente polar e aprótico dimetilsulfóxido (DMSO) (densidade $=1.08 \mathrm{~g} / \mathrm{cm}^{3}$, dipolo=4.48 D), e dois solventes polares e próticos metanol (densidade $=0.80 \mathrm{~g} / \mathrm{cm}^{3}$, dipolo $=2.27 \mathrm{D}$ ) e água (densidade $=1.00 \mathrm{~g} / \mathrm{cm}^{3}$, dipolo $\left.=2.27 \mathrm{D}\right)$. A seguir apresentamos os resultados da análise das simulações clássicas para cada solvente.

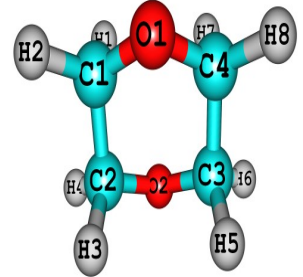

(a) 1,4 dioxano

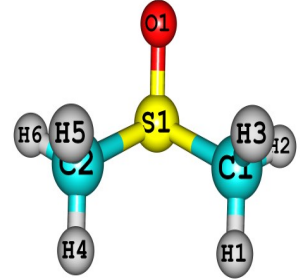

(b) DMSO

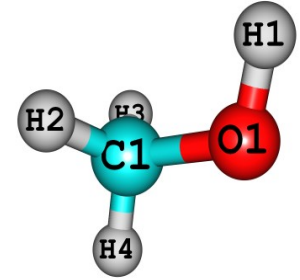

(c) Metanol

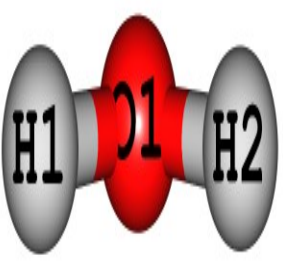

(d) Água

Figura 6.9: Ilustração dos solventes considerados nas simulações.

\section{1,4 dioxano e DMSO}

O 1,4 dioxano é um solvente que tem constante dielétrica igual a 2.21, para realizar a simulação foi considerada uma densidade inicial igual a $1.03 \mathrm{~g} / \mathrm{cm}^{3}$. DMSO é um solvente que tem constante dielétrica igual a 46.83 e foi considerada a densidade inicial igual a $1.08 \mathrm{~g} / \mathrm{cm}^{3}$. Para realizarmos a simulação clássica com Monte Carlo (MC) os parâmetros para os dois solventes foram obtidos pelo campo de força OPLSAA 123, 124. Em nossas simulações o número de partículas foi de 1 sonda e 500 moléculas do solvente, à pressão de 1 atm e temperatura de $298 \mathrm{~K}$. As simulações foram realizadas utilizando condições periódicas de contorno numa caixa cúbica de lado aproximadamente igual a $41.5 \AA$ (39.3 $\AA$ ) em 1,4 dioxano (DMSO). 
O primeiro passo feito na simulação foi a termalização do sistema (sonda + solvente) para garantirmos que esse esteja em equilíbrio termodinâmico. No processo de termalização foram realizados $3 \times 10^{7}$ passos $\mathrm{MC}$, o que equivale à 60.000 ciclos $\mathrm{MC}$, onde um ciclo se completa quando tentamos mover todas as moléculas da caixa de simulação. Na figura 6.10 apresentamos o comportamento da energia por molécula versus o número de ciclos para a sonda $\mathrm{MePMBr}_{2}$ solvatada em 1,4 dioxano durante o processo de termalização do sistema. Vê-se nessa figura que a energia por molécula atinge gradativamente o equilíbrio. O mesmo comportamento foi observado para as demais sondas estudadas nos outros solventes.

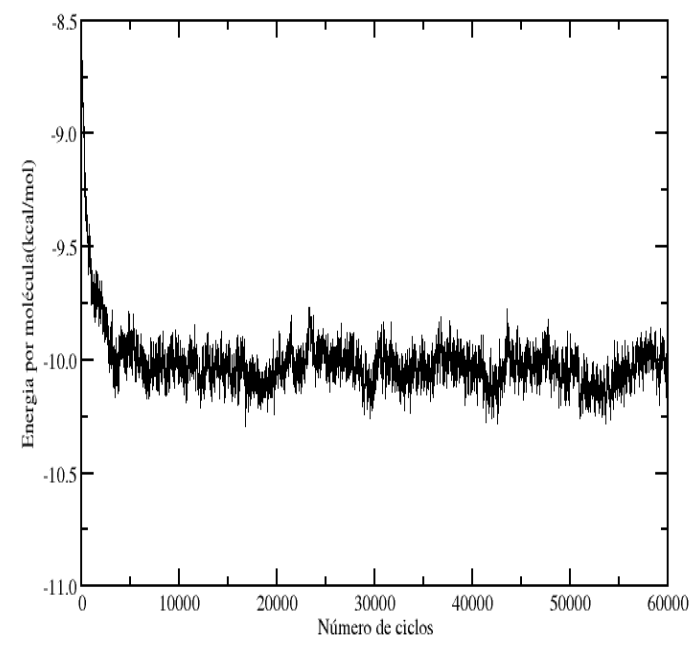

Figura 6.10: Energia por molécula versus o número de ciclos durante o processo de termalização do sistema. Dados obtidos para a sonda $\mathrm{MePMBr}_{2}$ em 1,4 dioxano.

Durante o processo de termalização acompanhamos também a equilibração de outras propriedades do sistema como a densidade e a energia de interação solutosolvente. Após a termalização, realizamos $6 \times 10^{7}$ passos $M C$, o que equivale à 120.000 ciclos MC. O comportamento da energia por molécula durante os ciclos MC é apresentado na figura 6.11. Observa-se que a energia flutua em torno de um valor médio 
caracterizando que o sistema está num estado de equilíbrio termodinâmico. O mesmo comportamento foi observado para as demais sondas estudadas nos outros solventes

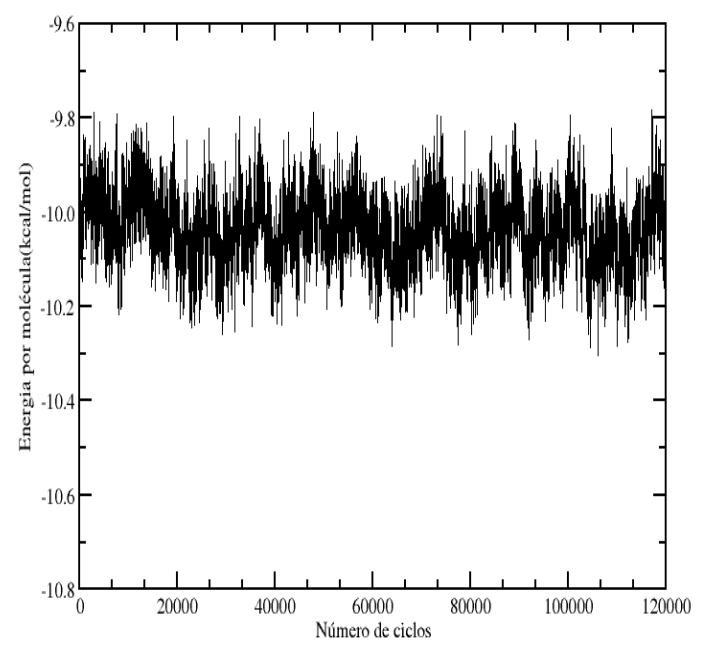

Figura 6.11: Energia por molécula versus o número de ciclos durante o equilíbrio termodinâmico do sistema. Dados obtidos para a sonda $\mathrm{MePMBr}_{2}$.

Realizadas às simulações, nós analisamos a distribuição do solvente em torno da molécula do soluto, com esse estudo podemos observar se existem as ligações de hidrogênio (LH) e determinar a quantidade de moléculas do solvente em cada camada de solvatação. A análise dessas quantidades é feita através da função de distribuição radial de pares $(\mathrm{RDF})$. Existem várias possibilidades para o cálculo da RDF, como a RDF entre o centro de massa do soluto e o centro de massa do solvente e a RDF calculada na mínima distância entre os átomos do soluto e os átomos do solvente, MDDF. Essas duas possibilidades citadas são mais usadas para a obtenção das camadas de solvatação. Na figura 6.12 analisamos a função radial de pares entre o oxigênio (O16) das sondas e o oxigênio (O1) dos solventes, são apresentados as análises para os dois solventes. A análise da RDF entre o oxigênio do soluto e o oxigênio do solvente, $\mathrm{G}_{O O}(\mathrm{r})$, é importante para obtenção da existência de ligações de hidrogênio. Nos casos 
estudados nesta seção, tanto o solvente quanto as moléculas do soluto possuem apenas átomos aceitadores de ligação de hidrogênio e portanto, no sistema soluto mais solvente não há formação de ligações de hidrogênio. Isso é refletido na função $\mathrm{G}_{O O}(\mathrm{r})$, figura 6.12, onde a distância mais provável entre o oxigênio do soluto e o oxigênio do solvente é de aproximadamente $4.4 \AA$, para o solvente 1,4 dioxano e $5.4 \AA$ para o DMSO.

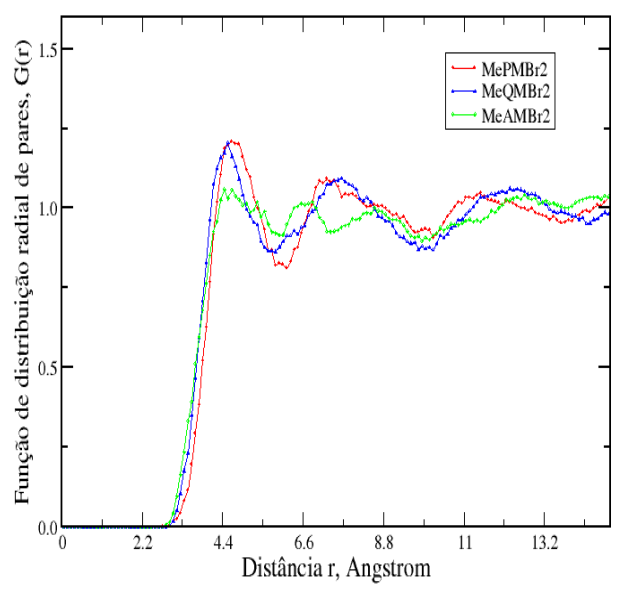

(a) 1,4 dioxano

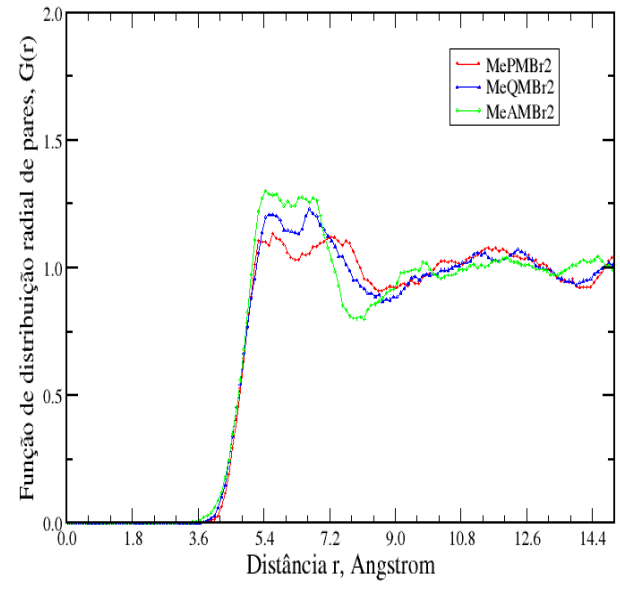

(b) DMSO

Figura 6.12: Função de distribuição radial de pares, $\mathrm{G}_{O O}(\mathrm{r})$, entre o oxigênio O16 das sondas e o oxigênio $\mathrm{O} 1$ dos solventes.

Uma etapa importante na análise da simulação é a obtenção da distribuição do solvente em torno do soluto, tal distribuição define as camadas de solvatação. Essa informação com relação ao número de moléculas do solvente que estão mais próximas do soluto será importante posteriormente pois nos permite escolher o tamanho do sistema que será tratado no cálculo quântico para obtenção do espectro das sondas em solução. Aqui, a informação das camadas de solvatação foi obtida analisando a RDF de mínima distância. Na figura 6.13 apresentamos a MDDF para as três sondas embebidas nos solventes 1,4 dioxano e DMSO. É possível observar três picos bem definidos para as sondas, um primeiro pico com fim em $4.0 \AA(4.2 \AA)$, o segundo com 
fim em $9.0 \AA(9.0 \AA)$ e um terceiro com fim em $12.6 \AA(12.0 \AA)$ em 1,4 dioxano (DMSO). A integração dessa função em cada um dos picos nos fornece o número de moléculas do solvente existente em cada camada de solvatação, na tabela 6.13 fazemos um sumário com todos os resultados obtidos para as três sondas. Os picos caracterizam as camadas de solvatação, onde o $1^{\circ}$ pico equivale à primeira camada de solvatação e assim para os demais. Observa-se na tabela 6.13 que a distribuição do solvente em torno das três sondas é similar. O limite da caixa possui 250 moléculas de solvente.

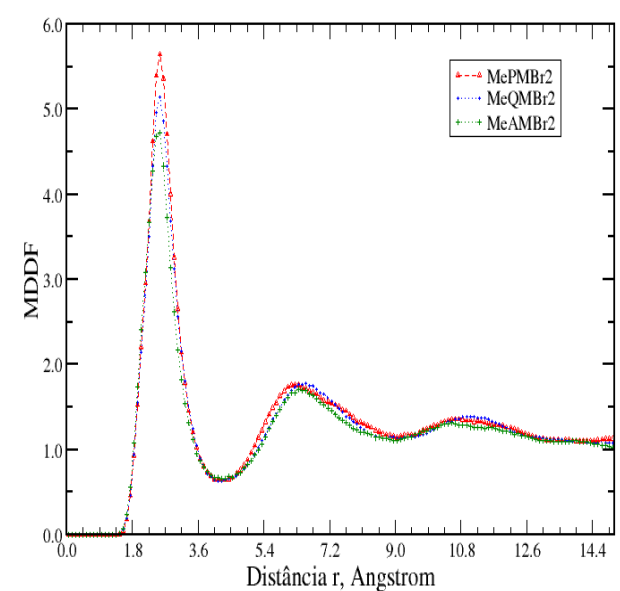

(a) 1,4 dioxano

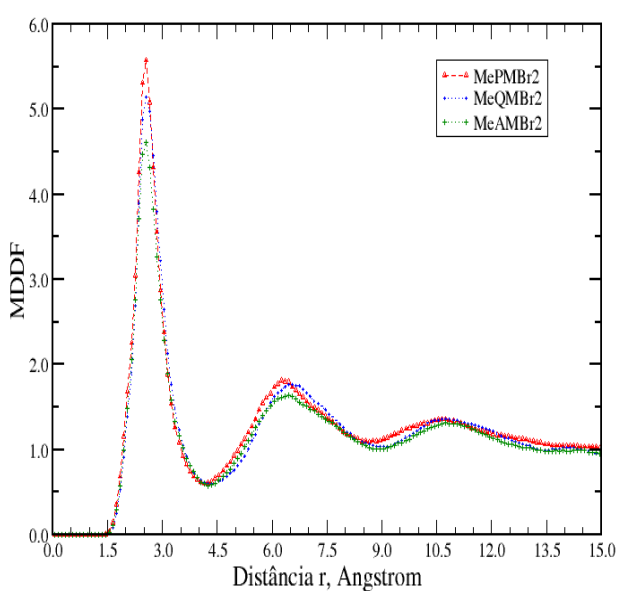

(b) DMSO

Figura 6.13: Função de distribuição de mínima distância entre o soluto e o solvente. 
Tabela 6.13: Número de moléculas do solvente 1,4 dioxano obtido até o final da camada de solvatação. Entre parênteses apresentamos os valores obtidos para o solvente DMSO.

\begin{tabular}{lccc}
\hline \hline Sonda & $1^{\circ}$ pico até $4.0 \AA(4.2 \AA)$ & $2^{\circ}$ pico até $9.0 \AA(9.0 \AA)$ & $3^{\circ}$ pico até $12.6 \AA(12.0 \AA)$ \\
\hline $\mathrm{MePMBr}_{2}$ & $23(25)$ & $90(99)$ & $181(183)$ \\
$\mathrm{MeQMBr}_{2}$ & $24(28)$ & $96(106)$ & $191(193)$ \\
$\mathrm{MeAMBr}_{2}$ & $27(30)$ & $102(112)$ & $200(202)$ \\
\hline \hline
\end{tabular}

\section{Metanol e Água}

O metanol é um solvente que tem constante dielétrica igual a 32.61, os parâmetros para descrever metanol foram obtidos do campo de força OPLS-AA [107. O outro solvente considerado foi a água, um solvente que tem constante dielétrica igual a 78.36. O modelo usado para a água foi o SPC [105]. A simulação foi realizada com o número de partículas igual a 1 molécula do soluto mais 1000 moléculas do solvente, a densidade inicial considerada para o metanol foi de $0.78 \mathrm{~g} / \mathrm{cm}^{3}$ e para a água foi 1 $\mathrm{g} / \mathrm{cm}^{3}$, à pressão de $1 \mathrm{~atm}$ e temperatura de $298 \mathrm{~K}$. Foram realizados 60.000 ciclos MC na termalização e 120.000 ciclos no equilíbrio. Atingido o equilíbrio do sistema nós analisamos as configurações geradas pela simulação para obtermos a distribuição do solvente ao redor do soluto. Ao contrário do que aconteceu com os solventes 1,4 dioxano e DMSO, há ligações de hidrogênio (LH) intermolecular formada entre as moléculas do solvente e a molécula do soluto.

Uma maneira para se estudar quantativamente as LHs é utilizando um critério energético e geométrico para definir quais os limites que caracterizam uma ligação de hidrogênio 125, 126. Essa combinação tem obtido êxito na descrição das LHs em líquidos 127, 128. Nesse procedimento é necessário definirmos os limites máximos 
para $\mathrm{R}_{A D}, \Theta_{A D H}$ e $\mathrm{E}_{l i g}$,onde $\mathrm{R}_{A D}$ é distância entre o átomo aceitador da ligação de hidrogênio e o átomo doador; $\Theta_{A D H}$ é o ângulo formado entre os átomos aceitador, doador e o hidrogênio ácido; e $\mathrm{E}_{\text {lig }}$ é o valor energético da ligação. Nesse caso, o átomo doador da ligação de hidrogênio é o oxigênio (O1) do solvente, metanol e água, ver figura 6.9 e o átomo aceitador é o oxigênio (O16) do soluto, ver figura 6.1. Sendo assim, temos que obter os valores limites para $\mathrm{R}_{O O}, \Theta_{O O H}$ e $\mathrm{E}_{l i g}$. A distância $\mathrm{R}_{O O}$ é obtida pelo primeiro mínimo da função radial de pares, $\mathrm{G}_{O O}(\mathrm{r})$.

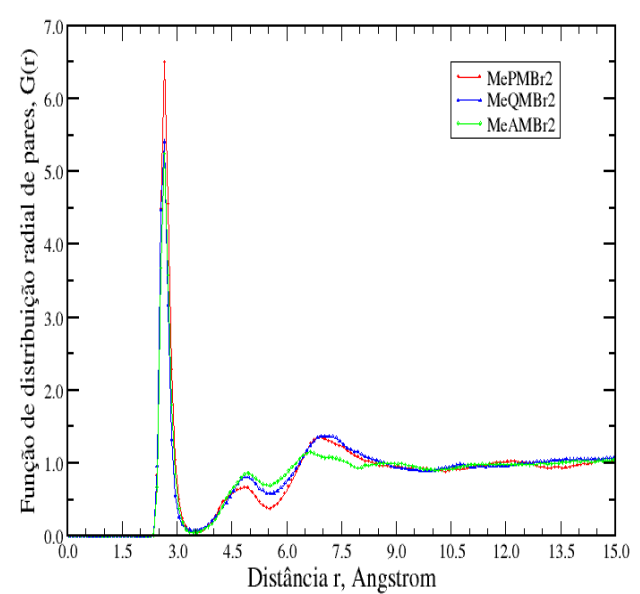

(a) metanol

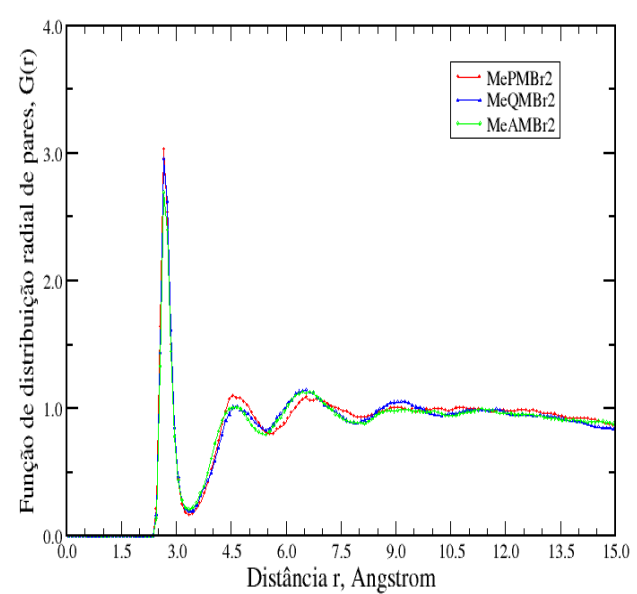

(b) água

Figura 6.14: Função de distribuição radial de pares, $\mathrm{G}_{O O}(\mathrm{r})$, entre o oxigênio $\mathrm{O} 16$ das sondas e o oxigênio O1 dos solventes.

Observa-se na figura 6.14 um pico bem pronunciado nos dois solventes com fim em $3.2 \AA$, que caracteriza as moléculas mais próxima do soluto. Essa distância será considerada como o nosso valor limite para o critério de distância, $\mathrm{R}_{O O}$. O valor limite para $\Theta_{O O H}$ foi de $40^{\circ}$, e para a energia de ligação, $\mathrm{E}_{l i g}$, definimos como qualquer valor negativo. Assim, na análise das configurações soluto-solvente geradas pela simulação nós consideramos uma ligação de hidrogênio quando a distância $\mathrm{R}_{O O}$ for menor que $3.2 \AA$, o ângulo entre o oxigênio da sonda e o grupo $\mathrm{OH}$ do metanol, $\Theta_{O O H}$, for 
menor do que $40^{\circ} \mathrm{e}$ a energia de ligação, $E_{\text {lig }}$ for negativa. Com esses critérios nós observamos que em média há 2 ligações de hidrogênio formadas com as moléculas do solvente, tanto para o metanol quanto para a água. Esse comportamento foi observado para as três sondas. Com relação às camadas de solvatação, nós também estudamos a MDDF. O comportamento dessa função é apresentado na figura 6.15. Observa-se que a solvatação das três sondas apresentam um comportamento semelhante nos dois solventes considerados. Na figura 6.15 vemos um primeiro pico com fim em $2.2 \AA(2.1$ $\AA$ ), que caracteriza as moléculas que estão fazendo ligações de hidrogênio em metanol (água). Há também um segundo pico com fim em $4.5 \AA(4.2 \AA)$, caracterizando a primeira camada de solvatação e um terceiro pico com fim em $7.5 \AA(7.2 \AA)$.

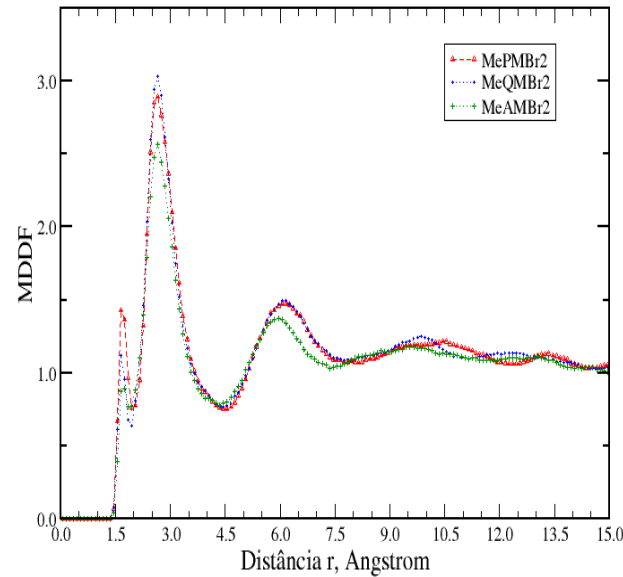

(a) metanol

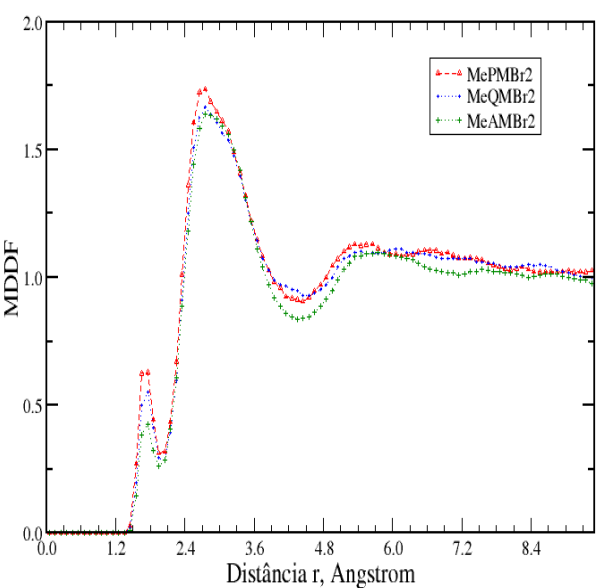

(b) água

Figura 6.15: Função de distribuição de mínima distância entre o soluto e o solvente.

Na tabela 6.14 apresentamos o número de moléculas do solvente existente em cada pico da MDDF. Esse valor é obtido pela integração dos picos da figura 6.15. O limite da caixa possui 500 moléculas de solvente. 
Tabela 6.14: Número de moléculas do solvente metanol obtido até o final da camada de solvatação. Entre parênteses apresentamos os valores obtidos para o solvente água.

\begin{tabular}{lccc}
\hline \hline Sonda & $1^{\mathrm{o}}$ pico até $2.2 \AA(2.1 \AA)$ & $2^{\circ}$ pico até $4.5 \AA(4.2 \AA)$ & $3^{\circ}$ pico até $7.5 \AA(7.2 \AA)$ \\
\hline $\mathrm{MePMBr}_{2}$ & $5(4)$ & $36(59)$ & $102(183)$ \\
$\mathrm{MeQMBr}_{2}$ & $5(4)$ & $40(65)$ & $112(197)$ \\
$\mathrm{MeAMBr}_{2}$ & $6(4)$ & $46(72)$ & $117(212)$ \\
\hline \hline
\end{tabular}

- Espectro eletrônico de absorção

O estudo do efeito do solvente no espectro de absorção das sondas com o modelo discreto para os solventes, foi feito usando o método sequencial QM/MM, primeiro as simulações com mecânica molecular e posteriormente os cálculos com mecânica quântica. Os cálculos quânticos foram feitos sobre as configurações estatísticamente descorrelacionadas obtidas das simulações, num total de 120 configurações para cada solvente. Na tabela 6.15 apresentamos os resultados para o espectro de absorção, onde foi considerada uma configuração eletrostática média do solvente, ASEC 106. Essa configuração média é gerada superpondo todas as configurações estatísticamente descorrelacionadas e dividindo as cargas pelo número de configurações utilizadas. O resultado obtido com essa configuração média é igual ao obtido quando fazemos a média sobre os resultados de $\mathrm{N}$ configurações com a mesma quantidade de moléculas do solvente [106]. Para obtermos as energias eletrônicas de absorção, nós realizamos QM usando o método TD-DFT onde foi utilizado os funcionais de densidade, CAMB3LYP e B3LYP com a função base utilizada foi $6-311+\mathrm{G}^{* *}$. 
Tabela 6.15: Valores para energia da transição $\pi-\pi^{*}\left(\mathrm{~cm}^{-1}\right)$ para as sondas estudadas. Valores teóricos obtidos com TD-DFT usando os funcional [CAM-B3LYP]B3LYP e as funções base $6-311+\mathrm{G}^{* *}$. Realizado na configuração ASEC para o respectivo solvente.

\begin{tabular}{|c|c|c|c|c|}
\hline Solventes & $\mathrm{E}\left(\mathrm{cm}^{-1}\right)$ & $\Delta E$ & $\mathrm{E}$ & $\Delta E$ \\
\hline & $\mathrm{MePMBr}_{2}$ & & Experimental $|30|$ & \\
\hline 1,4 dioxano & {$[21982] 21471$} & 0 & 15980 & 0 \\
\hline Metanol & [23998]22229 & {$[2016] 757$} & 20719 & 4739 \\
\hline DMSO & {$[23723] 22143$} & {$[1741] 672$} & 18680 & 2700 \\
\hline \multirow[t]{2}{*}{ Água } & {$[25231] 23165$} & {$[3249] 1694$} & 22815 & 6835 \\
\hline & $\mathrm{MeQMBr}_{2}$ & & Experimental |30| & \\
\hline 1,4 dioxano & {$[20370] 19478$} & 0 & 14476 & 0 \\
\hline Metanol & {$[21549] 20065$} & {$[1179] 586$} & 17879 & 3403 \\
\hline DMSO & {$[21664] 20229$} & {$[1294] 751$} & 15970 & 1494 \\
\hline Água & {$[22799] 21038$} & {$[2429] 1560$} & 20842 & 6366 \\
\hline $\mathrm{MeAMBr}_{2}$ & & Experimental |30| & & \\
\hline 1,4 dioxano & {$[20240] 18942$} & 0 & 16225 & 0 \\
\hline Metanol & [19338]18372 & {$[-902]-570$} & 14403 & -1822 \\
\hline DMSO & [19354]18416 & {$[-886]-526$} & 13913 & -2312 \\
\hline Água & {$[19614] 18503$} & {$[-626]-439$} & 17822 & 1597 \\
\hline
\end{tabular}


Observa-se na tabela 6.15 que os funcionais B3LYP e CAM-B3LYP descrevem corretamente a tendência dos deslocamentos solvatocrômicos observados experimentalmente, mas os valores calculados não estão em concordância com os valores experimentais absolutos, com exceção dos valores calculados com B3LYP para a solução aquosa que apresenta uma boa concordância. Nessa tabela também é interessante ressaltar que: os valores das transições eletrônicas calculados para as sondas no solvente 1,4-dioxano (solvente de baixa polaridade próximo à polaridade do benzeno) são discrepantes e isso indica que a geometria que temos para descrever o estado fundamental não está correta ou que o método que estamos utilizando não descreve corretamente o tipo de transição calculada. Com relação a geometria, investigamos as várias formas que as sondas podem assumir: cis, trans, zwiteriônica e protonada. Também realizamos dinâmica molecular ab initio em superfície de Born-Oppenheimer (BOMD) em vácuo, o nível de cálculo quântico utilizado para obter o estado fundamental de referência foi Hartree Fock (HF) com o conjunto de funções base 6-31+ $\mathrm{G}^{*}$. A dinâmica transcorreu à temperatura de $298 \mathrm{~K}$ e foram realizados 100 passos resultando numa trajetória de 0.4 fs. Foram usadas as mesmas condições da dinâmica para as três sondas estudadas. Identificamos que as deformações moleculares contribuem para um alargamento da banda do espectro de absorção mas não é capaz de deslocar os valores das transições eletrônicas calculadas para os valores medidos experimentalmente. Com relação aos métodos, investigamos vários funcionais diferentes e outros métodos como EOMCCSD e TDDFT(B3LYP, BHandHLYP, CAM-B3LYP, LC-WPBE, WB97XD). Como mencionado anteiormente, o método EOMCCSD é o estado da arte para o cálculo das transições eletrônicas e por isso tem-se grande confiança nesse valor. Sendo assim, acreditamos que a discrepância entre o valor calculado para a excitação eletrônica em vácuo e o valor medido experimentalmente é oriundo de alguma hipótese que ainda não foi investigada. Uma hipótese levantada neste trabalho é a de que em solventes de baixa polaridade existiria agregação ou dimerização. 
Desta forma, a transição eletrônica seria descrita por agregados da sonda e não por moléculas isoladas em solução.

\subsection{Dímeros em solução}

No estudo dos dímeros, nós otimizamos a geometria das sondas dimerizadas embebidas num meio contínuo PCM, o solvente considerado nesta primeira etapa no estudo de agregados foi o de mais baixa polaridade, 1, 4 dioxano. As geometrias dos dímeros foram obtidas com o nível de cálculo B3LYP $/ 6-311+G^{* *}$. Na figura 6.16 apresentamos uma ilustração dos dímeros estudados.
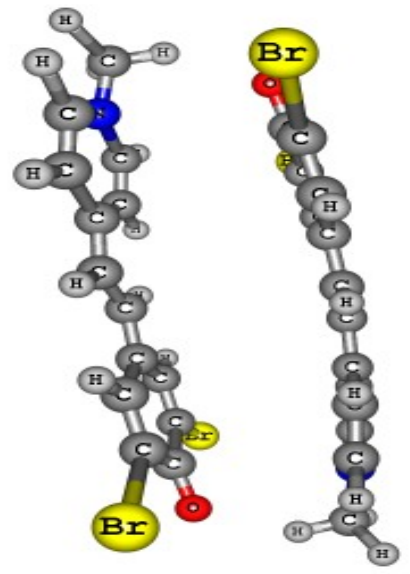

(a) $\mathrm{MePMBr}_{2}$

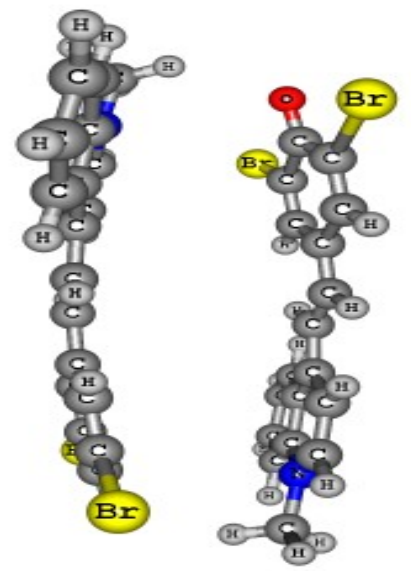

(b) $\mathrm{MeQMBr}_{2}$

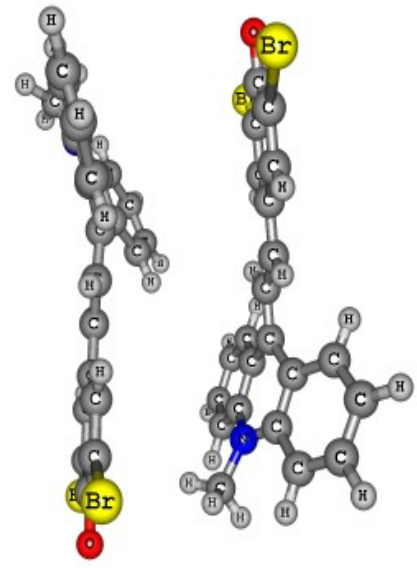

(c) $\mathrm{MeAMBr}_{2}$

Figura 6.16: Ilustração dos dímeros antiparalelos otimizados das sondas.

A energia de ligação dos dímeros em PCM foi obtida. Nós obtemos um valor de $15.09 \mathrm{kcal} / \mathrm{mol}$ para a sonda $\mathrm{MePMBr}_{2},-10.84 \mathrm{kcal} / \mathrm{mol}$ para a sonda $\mathrm{MeQMBr}_{2}$ e -4.09 $\mathrm{kcal} / \mathrm{mol}$ para a sonda $\mathrm{MeAMBr}_{2}$. Esses valores mostram a estabilidade na formação dos dímeros. Nós analisamos também a deformação na estrutura dos monômeros provocadas pela dimerização. 
Tabela 6.16: Valores para o comprimento da ligação $(\AA)$ e angulos diedros $\left(^{\circ}\right)$ para os monômeros obtidos da formação do dímero. Geometria do dímero otimizada no nível B3LYP /6-311 $+\mathrm{G}^{* *}$ embebida no solvente 1,4 dioxano com PCM.

\begin{tabular}{cccc}
\hline \hline Propriedades & $\mathrm{MePMBr}_{2}$ & $\mathrm{MeQMBr}_{2}$ & $\mathrm{MeAMBr}_{2}$ \\
\hline $\mathrm{R}(\mathrm{C} 8-\mathrm{C} 9)$ & 1.376 & 1.383 & 1.400 \\
$\mathrm{R}(\mathrm{C} 15-\mathrm{O} 16)$ & 1.243 & 1.239 & 1.230 \\
$\Phi(\mathrm{C} 4-\mathrm{C} 3-\mathrm{C} 8-\mathrm{C} 9)$ & 1.102 & -4.833 & 164.456 \\
$\Phi(\mathrm{C} 8-\mathrm{C} 9-\mathrm{C} 10-\mathrm{C} 12)$ & -1.147 & -1.376 & 175.796 \\
$\Phi(\mathrm{C} 3-\mathrm{C} 8-\mathrm{C} 9-\mathrm{C} 10)$ & 172.815 & 172.211 & 176.713 \\
$\Phi(\mathrm{C} 10-\mathrm{C} 12-\mathrm{C} 14-\mathrm{C} 15)$ & 0.768 & 0.755 & -0.138 \\
\hline \hline
\end{tabular}

No geral, observamos que há uma pequena deformação do monômero quando otimizado formando dímero, isso pode ser percebido comparando a tabela 6.16 com a tabela 6.1. A planaridade das sondas $\mathrm{MePMBr}_{2}$ e $\mathrm{MeQMBr}_{2}$ é mantida, ver diedro $\Phi(\mathrm{C} 4-\mathrm{C} 3-\mathrm{C} 8-\mathrm{C} 9)$, e a sonda $\mathrm{MeAMBr}_{2}$ continua não planar. Há pequenas mudanças nas ligações C8-C9 (menor nos dímeros) e C15-O16 (maior nos dímeros).

Para o espectro de absorção, nós calculamos a energia da transição eletrônica para os dímeros isolados. Nós realizamos cálculos com dois funcionais DFT, CAMB3LYP e B3LYP, com as funções base $6-311+\mathrm{G}^{* *}$. Na tabela 6.17 apresentamos os nossos resultados para a transição eletrônica dos dímeros. Os agregados apresentam uma transição de mais baixa energia, na região da transição obtida experimentalmente, e outra transição na região de $20000 \mathrm{~cm}^{-1}$, que é referente a transição eletrônica dos monômeros isolados. Observa-se que devido a formação do dímero surge uma transição de baixa energia na região observada experimentalmente, mas ainda temos transições eletrônicas referentes aos monômeros. 
Tabela 6.17: Valores para energia da transição $\pi-\pi^{*}, \mathrm{E}\left(\mathrm{cm}^{-1}\right)$, e comprimento de onda, $\lambda_{\max }$ $(\mathrm{nm})$, para os dímeros isolados. Valores teóricos obtidos com TD-DFT usando os funcional B3LYP e as funções base 6-311+ $\mathrm{G}^{* *}$ calculados em 1,4 dioxano usando PCM.

\begin{tabular}{|c|c|c|c|}
\hline \multirow[t]{2}{*}{ Sonda } & $\mathrm{E}$ & $\mathrm{E}$ & Experimental $\mid 30$ \\
\hline & $\left(\lambda_{\max }\right)$ & $\left(\lambda_{\max }\right)$ & $\left(\lambda_{\max }\right)$ \\
\hline \multirow[t]{5}{*}{$\left(\mathrm{MePMBr}_{2}\right)_{2}$} & CAM-B3LYP & B3LYP & \\
\hline & 19286 & 15443 & 15980 \\
\hline & $(519)$ & $(647)$ & $(626)$ \\
\hline & 23903 & 22738 & \\
\hline & $(418)$ & $(440)$ & \\
\hline \multirow[t]{5}{*}{$\left(\mathrm{MeQMBr}_{2}\right)_{2}$} & CAM-B3LYP & B3LYP & \\
\hline & 17822 & 14369 & 14476 \\
\hline & $(561)$ & $(696)$ & $(691)$ \\
\hline & 21825 & 20610 & \\
\hline & $(458)$ & $(485)$ & \\
\hline \multirow[t]{5}{*}{$\left(\mathrm{MeQMBr}_{2}\right)_{2}$} & CAM-B3LYP & B3LYP & \\
\hline & 18343 & 14298 & 16225 \\
\hline & $(545)$ & $(699)$ & $(616)$ \\
\hline & 21872 & 22330 & \\
\hline & $(457)$ & $(449)$ & \\
\hline
\end{tabular}


Com relação aos orbitais envolvidos na transição, observa-se na ilustração dos orbitais, figuras 6.17, 6.18 6.19, que há uma transferência de carga entre os monômeros. A transição para os monômeros separados também foi calculada para verificarmos se o deslocamento solvatocrômico era devido apenas à deformação da geometria provocada pela formação dos dímeros. O valor da transição eletrônica para os monômeros é apresentado na tabela 6.18 . Observa-se que a transição eletrônica para os monômeros isolados estão deslocados do valor experimental, o que indica que as transições de baixa energia apresentadas na tabela 6.17 são oriundas da formação do dímero.

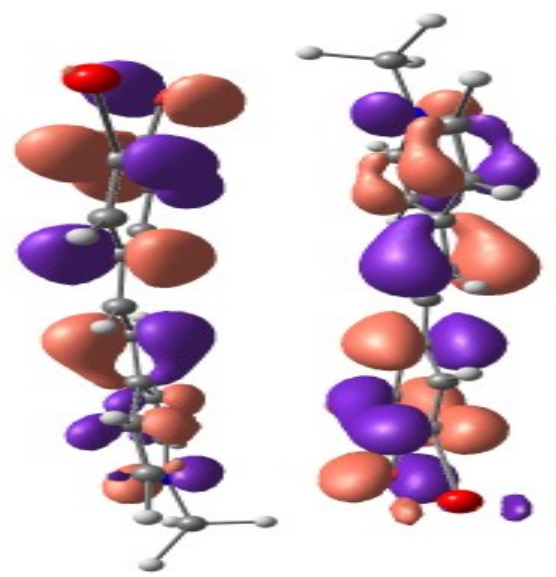

(a) HOMO

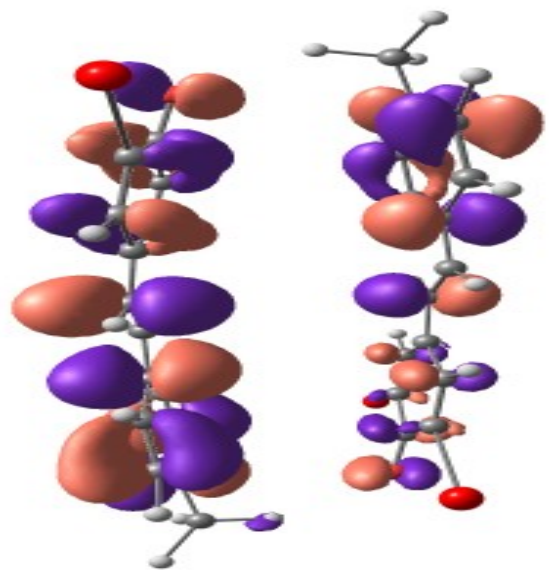

(b) LUMO

Figura 6.17: Ilustração dos orbitais moleculares envolvidos na transição eletrônica obtidos com TD-B3LYP /6-311 $+\mathrm{G}^{* *}$ para o dímero de $\mathrm{MePMBr}_{2}$. 

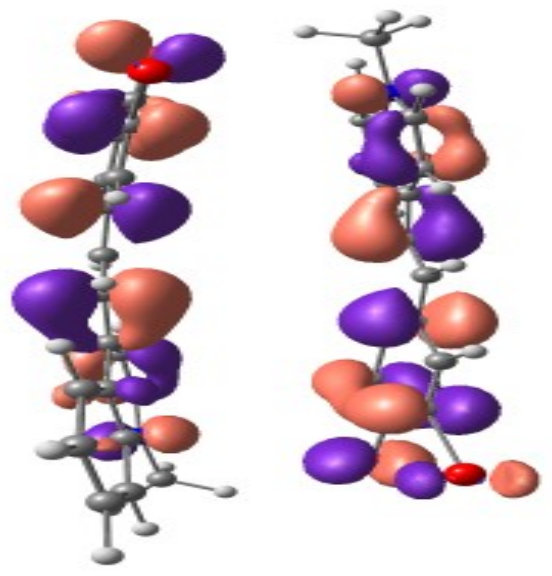

(a) HOMO
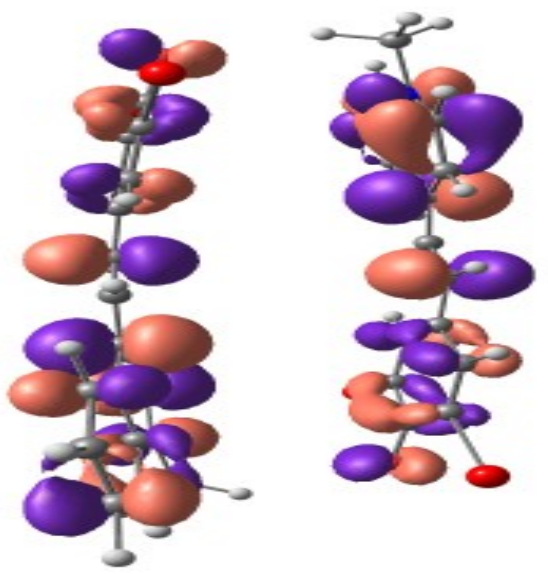

(b) LUMO

Figura 6.18: Ilustração dos orbitais moleculares envolvidos na transição eletrônica obtidos com TD-B3LYP/6-311+G** para o dímero de $\mathrm{MeQMBr}_{2}$.
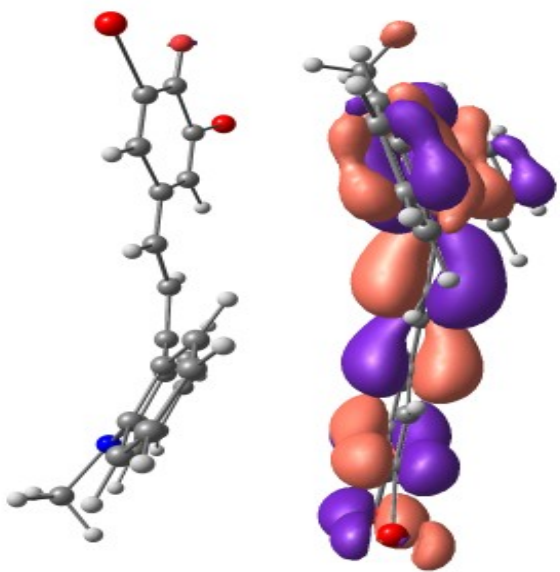

(a) HOMO

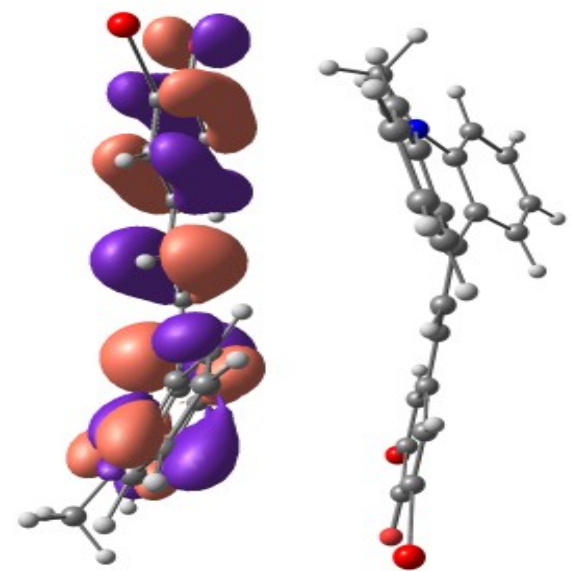

(b) LUMO

Figura 6.19: Ilustração dos orbitais moleculares envolvidos na transição eletrônica obtidos com TD-B3LYP/6-311+G** para o dímero de $\mathrm{MeAMBr}_{2}$. 
Tabela 6.18: Valores para energia da transição $\pi-\pi^{*}\left(\mathrm{~cm}^{-1}\right)$ para os monômeros isolados. Valores teóricos obtidos com TD-DFT usando os funcional B3LYP (CAM-B3LYP) e as funções base $6-311+\mathrm{G}^{* *}$.

\begin{tabular}{lccc}
\hline \hline Sonda & $\mathrm{E}\left(\mathrm{cm}^{-1}\right)$ & $\mathrm{E}\left(\mathrm{cm}^{-1}\right)$ & Experimental $|30|$ \\
\hline & Monômero 1 & Monômero 2 & \\
$\mathrm{MePMBr}_{2}$ & $20366(21116)$ & $20423(21132)$ & 15980 \\
$\mathrm{MeQMBr}_{2}$ & $18778(19777)$ & $18778(19778)$ & 14476 \\
$\mathrm{MeAMBr}_{2}$ & $18707(19898)$ & $18890(20046)$ & 16225 \\
\hline \hline
\end{tabular}

\subsubsection{Simulação com dímeros}

No estudo dos dímeros, nós realizamos simulações clássicas usando o método Monte Carlo no solvente 1,4 dioxano. As geometrias dos dímeros foram otimizadas na presença do solvente, usando o modelo PCM, com o nível de cálculo B3LYP/6$311+\mathrm{G}^{* *}$. O procedimento para obtenção dos parâmetros do potencial de LennardJones é similar ao descrito na seção 6.2. O conjunto de cargas utilizado foi o obtido para os monômeros em 1,4 dioxano. As simulações foram realizadas no ensemble NPT. O número de partículas foi de 1 dímero e 500 moléculas do solvente (1,4 dioxano), à pressão de 1 atm e temperatura de $298 \mathrm{~K}$. Na termalização foram gerados 60.000 ciclos MC e na etapa de produção 120.000 ciclos MC.

Inicialmente, na simulação, os monômeros estão próximos formando dímero antiparalelo. O nosso interesse é verificar se a formação de dímero é estável e continua ao longo da simulação em meio solvente. Para realizar essa análise nós obtivemos a função de distribuição radial de pares, RDF, entre os átomos do soluto. Fizemos isso entre os dois átomos de nitrogênio dos dois monômeros, $\mathrm{G}_{N N}(r)$, e entre o nitrogênio 
de um monômero com o oxigênio do outro, $\mathrm{G}_{N O}(r)$. Essas funções estão apresentadas na figura 6.20 .

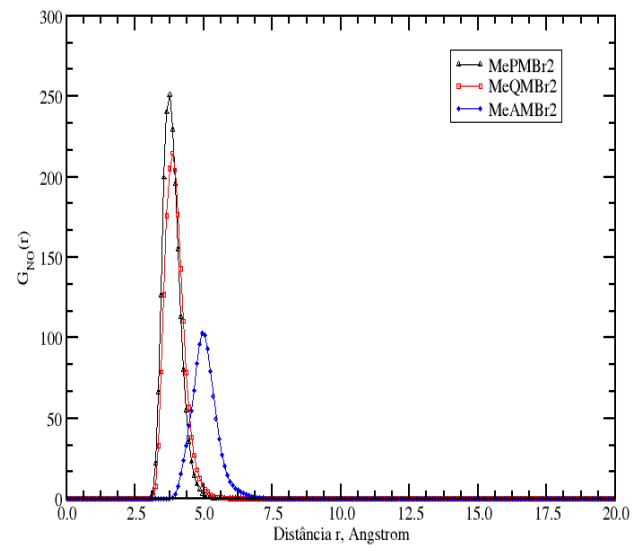

(a) $\mathrm{G}_{N O}(r)$

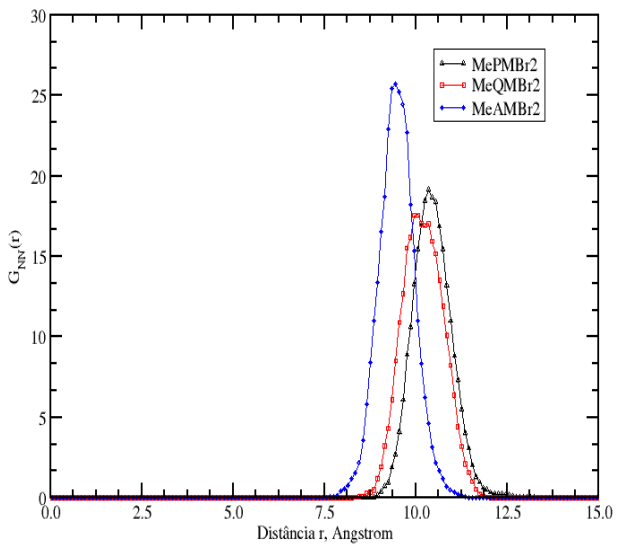

(b) $\mathrm{G}_{N N}(r)$

Figura 6.20: Função de distribuição radial de pares, G(r).

Observa-se na figura 6.20 um pico pronunciado em torno de $4 \AA$ para as sondas menores e $5 \AA$ para a sonda maior $\left(\mathrm{MeAMBr}_{2}\right)$, para a $\mathrm{G}_{N O}(r)$, e um pico em torno de $10 \AA$ para a $\mathrm{G}_{N N}(r)$, caracterizando que não há moléculas de solvente entre os monômeros.

A energia de interação entre os monômeros também pode ser analisada, na figura 6.21 apresentamos o resultado obtido para a sonda $\mathrm{MePMBr}_{2}$, observa-se na figura que a energia de interação entre os monômeros é sempre negativa, ao longo da simulação de equilíbrio, e bastante forte com um valor médio de $-30.5 \pm 2.2 \mathrm{kcal} / \mathrm{mol}$. Isso mostra que a formação do dímero é mantida. O mesmo comportamento foi obtido para as demais sondas e os valores médios para a energia de ligação dos dímeros ao longo da simulação são $-24.4 \pm 1.1 \mathrm{kcal} / \mathrm{mol}$ para $\left(\mathrm{MeQMBr}_{2}\right)_{2}$ e $-19.8 \pm 1.0 \mathrm{kcal} / \mathrm{mol}$ para $\left(\mathrm{MeAMBr}_{2}\right)_{2}$ em 1,4 dioxano. 


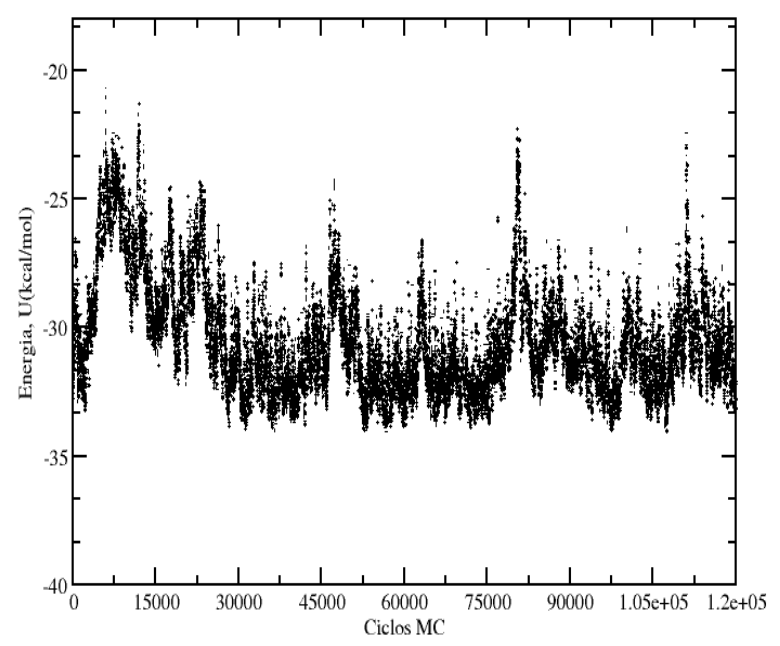

Figura 6.21: Energia de interação entre as duas moléculas do soluto, $\mathrm{U}(\mathrm{kcal} / \mathrm{mol})$, ao longo da etapa de produção para a sonda $\mathrm{MePMBr}_{2}$.

Verificado que o dímero é estável ao longo da simulação, nós realizamos cálculos nas configurações geradas na simulação para obtermos o espectro de absorção dos agregados em solução. Os valores foram obtidos com TD-DFT usando o nível de cálculo B3LYP $/ 6-311+\mathrm{G}^{* *}$ e com o solvente tratado através de cargas pontuais. Na tabela 6.19 apresentamos o valor médio obtido pelos cálculos nas configurações (selecionamos 100 configurações descorrelacionadas) dos dímeros solvatados em 1,4 dioxano. Observa-se que o valor para a transição eletrônica de mais baixa energia dos dímeros apresenta uma melhor concordância com o valor experimental. Indicando que em solventes de baixa polaridade há formação de dímeros nas sondas estudadas. 
Tabela 6.19: Valores para energia da transição $\pi-\pi^{*}, \mathrm{E}\left(\mathrm{cm}^{-1}\right)$, e comprimento de onda, $\lambda_{\max }(\mathrm{nm})$, para os dímeros em 1,4 dioxano descrito como cargas pontuais. Valores teóricos obtidos com TD-DFT usando os funcional B3LYP e as funções base 6-311+G**.

\begin{tabular}{lcc}
\hline \hline Sonda & $\mathrm{E}$ & Experimental $|30|$ \\
& $\left(\lambda_{\text {max }}\right)$ & $\left(\lambda_{\text {max }}\right)$ \\
\hline$\left(\mathrm{MePMBr}_{2}\right)_{2}$ & $15108 \pm 565$ & 15980 \\
& $(662 \pm 25)$ & $(626)$ \\
$\left(\mathrm{MeQMBr}_{2}\right)_{2}$ & $14032 \pm 633$ & 14476 \\
& $(713 \pm 32)$ & $(691)$ \\
$\left(\mathrm{MeAMBr}_{2}\right)_{2}$ & $14331 \pm 577$ & 16225 \\
& $(698 \pm 28)$ & $(616)$ \\
\hline \hline
\end{tabular}




\section{Capítulo 7}

\section{Merocianina de Brooker: Resultados experimentais}

Neste capítulo apresentaremos os resultados das medidas experimentais da sonda MB. Fizemos um estudo do efeito do solvente no espectro de absorção da sonda em diferentes solventes, foram eles: água, metanol, etanol, DMSO, acetonitrila e clorofórmio. Variando o pH das soluções em água e metanol, obtivemos a curva de titulação e determinamos o $p K_{a}$ da MB nesses solventes. Variando a concentração do soluto, analisamos a autoagregação da MB em diferentes solventes: água, metanol e acetonitrila.

\subsection{Estudo do deslocamento solvatocrômico da Me- rocianina de Brooker}

Nesta seção vamos analisar o efeito de diferentes solventes no espectro de absorção eletrônico da sonda MB. Os resultados aqui apresentados foram obtidos através de medidas experimentais. Os solventes considerados foram: água, metanol, etanol, DMSO, acetonitrila e clorofórmio. A concentração da sonda foi de $0.015 \mathrm{mM}$ para todos os solventes exceto o clorofórmio, pois nesse solvente não houve uma diluição completa do filme obtido após o procedimento de secagem da alíquota da solução estoque. Na 
figura 7.1 apresentamos a curva experimental obtida para os diferentes solventes.

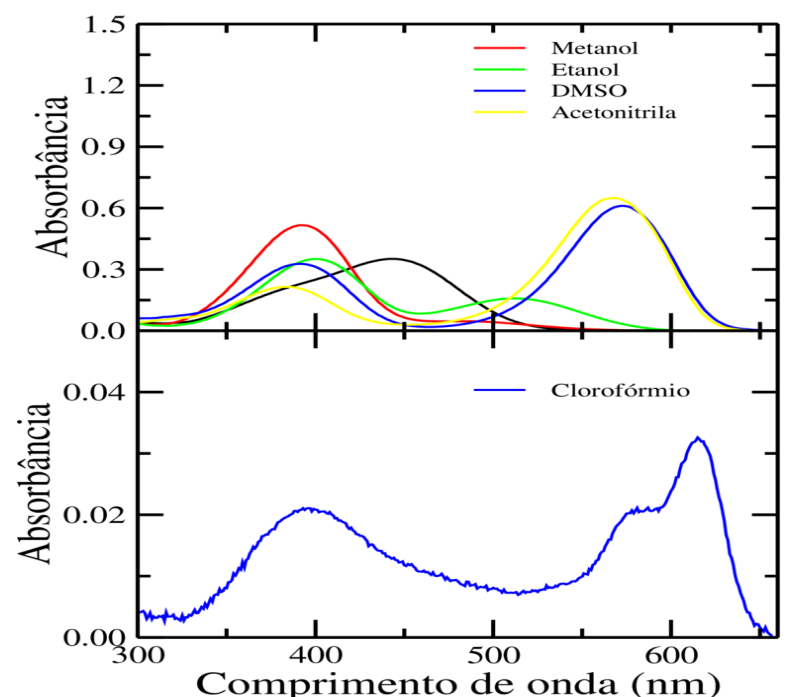

Figura 7.1: Espectro eletrônico de absorção da sonda MB medido em diferentes solventes.

Observa-se na figura 7.1 no espectro de absorção que há duas bandas bem definidas que estão associadas às formas neutra e protonada da sonda. Uma banda com menor comprimento de onda, $\lambda_{1}$, associada a forma protonada $\mathrm{MBH}^{+}$e outra banda com maior comprimento de onda, $\lambda_{2}$ associada a forma neutra MB. Os valores obtidos para cada solvente estão apresentado na tabela 7.1 e comparados com os valores da literatura. A boa concordância dos nossos valores medidos com os valores da literatura demonstra a qualidade da amostra da MB que foi cedida pelo nosso colaborador experimental [32]. 
Tabela 7.1: Valores para o comprimento de onda de máxima absorção, $\lambda_{\max }$, da primeira e segunda bandas de absorção obtidos da curva apresentada na figura 7.1. Em parênteses apresentamos os valores referentes ao ombro da primeira banda no solvente clorofórmio. $\mathrm{O}$ erro experimental é de $1 \mathrm{~nm}$.

\begin{tabular}{|c|c|c|}
\hline Solvente & $\lambda_{1}(n m)$ & $\lambda_{2}(n m)$ \\
\hline & protonada $\mathrm{MBH}^{+}$ & desprotonada $M B$ \\
\hline Água & 371 & 444 \\
\hline Outros autores & $370|33|$ & $444|32,33|, 442|22,34,35|, 443|36|$ \\
\hline Metanol & 390 & 485 \\
\hline Outros autores & & $487|32|, 483|22| ,36|, 484| 34|, 485| 35|, 490| 37 \mid$ \\
\hline Etanol & 400 & 513 \\
\hline Outros autores & & $513|32|$ \\
\hline DMSO & 391 & 573 \\
\hline Outros autores & & $576|32|, 573|34|, 575|36|$ \\
\hline Acetonitrila & 382 & 567 \\
\hline Outros autores & & $572|32|, 571|22|, 574|34|, 568|35,36|$ \\
\hline Clorofórmio & 397 & $615(581)$ \\
\hline Outros autores & & $620|22,32|, 613|36|, 617(578)|35|, 618(589) \mid 37$ \\
\hline
\end{tabular}


Há diversos trabalhos na literatura que relatam o solvatocromismo apresentado pela sonda MB [22, 34, 37], onde é discutida apenas a banda de maior comprimento de onda (ou menor energia) que é correspondente a forma neutra da molécula. Há trabalhos que relatam que a estrutura da sonda MB possui tanto a forma neutra quanto a forma protonada $\left(\mathrm{MBH}^{+}\right)$, e essa mudança estrutural é dependente do $\mathrm{pH}$ da solução [33|. Para a nossa medida experimental, como foi relatado na seção de materias e métodos, a solução estoque pura foi produzida numa mistura de metanol/etanol, nessa situação a cristalização da sonda a partir da solução estoque poderia conter populações das duas formas (neutra/protonada). Dessa forma, as duas bandas apresentadas na figura 7.1 corresponderiam às absorbâncias das duas estruturas diferentes da sonda MB. Buscando observar o comportamento de cada uma das estruturas separadamente, nós preparamos duas soluções estoques diferentes. Uma solução estoque de metanol ácido $\left(\mathrm{pH}_{a p}=0.0\right)$ e uma solução de metanol alcalina $\left(\mathrm{pH}_{a p}=13.3\right)$. Nessas condições nós podemos garantir que há apenas a população de uma dada estrutura em cada solução estoque. Preparada as soluções, nós realizamos a medida do espectro de absorção da sonda para os diferentes solventes apresentados na tabela 7.1 . Os resultados dessas medidas estão apresentados nas figuras 7.2 (solução ácida) e 7.3 (solução básica). 


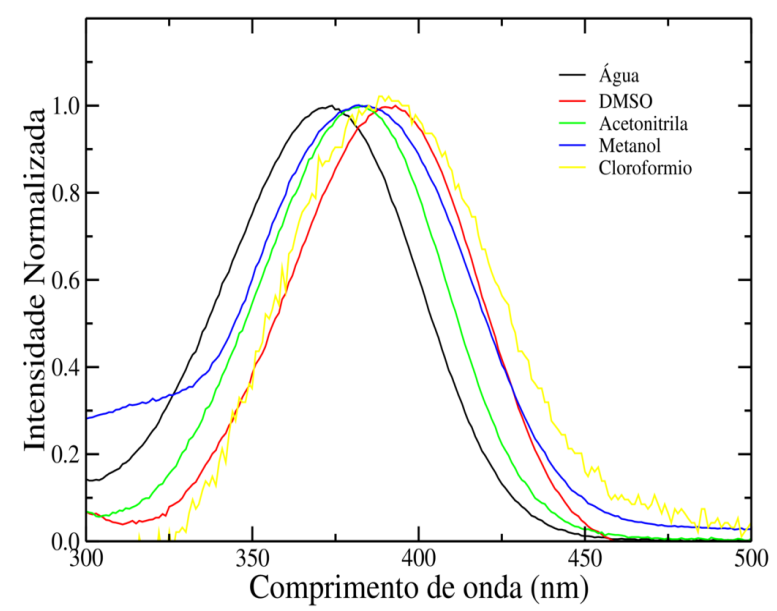

Figura 7.2: Medidas para a absorbância normalizada da sonda MB em diferentes solventes utilizando a solução estoque ácida. Os solventes utilizados foram: água $\left(\lambda_{\max }=374 \mathrm{~nm}\right)$, $\operatorname{DMSO}\left(\lambda_{\max }=393 \mathrm{~nm}\right)$, acetonitrila $\left(\lambda_{\max }=383 \mathrm{~nm}\right)$, metanol $\left(\lambda_{\max }=382 \mathrm{~nm}\right)$ e clorofórmio $\left(\lambda_{\max }=397 \mathrm{~nm}\right)$.

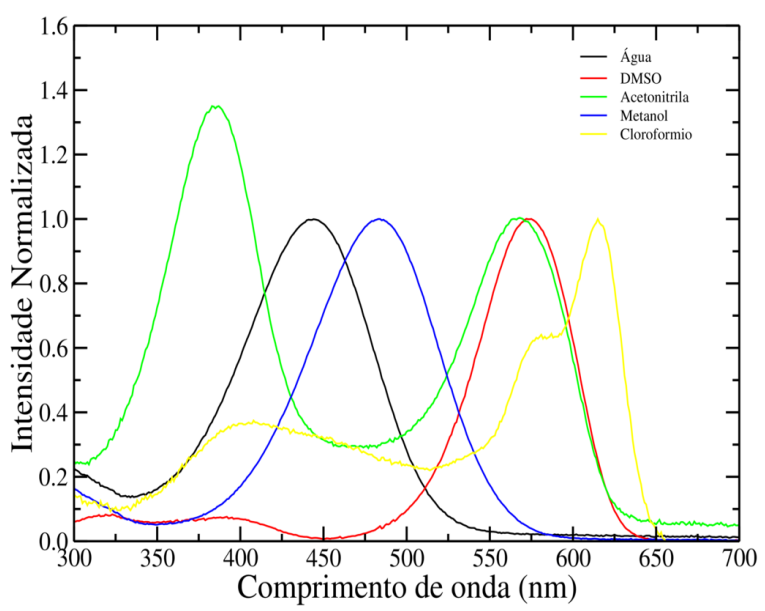

Figura 7.3: Medidas para a absorbância normalizada da sonda MB em diferentes solventes utilizando a solução estoque alcalina. Os solventes utilizados foram: água $\left(\lambda_{\max }=444 \mathrm{~nm}\right)$, DMSO $\left(\lambda_{\max }=572\right.$ e $\left.391 \mathrm{~nm}\right)$, acetonitrila $\left(\lambda_{\max }=568\right.$ e $\left.387 \mathrm{~nm}\right)$, metanol $\left(\lambda_{\max }=484 \mathrm{~nm}\right)$ e clorofórmio $\left(\lambda_{\max }=615\right.$ e $\left.408 \mathrm{~nm}\right)$.

Observa-se que o espectro de absorção da sonda MB é bastante sensível também ao pH da solução. A solução estoque ácida é caracterizada por uma banda que apresenta absorbância máxima entre 380 e $400 \mathrm{~nm}$, ver figura 7.2. Por outro lado, a solução alcalina apresenta uma absorbância máxima entre 440 e 620 nm, ver figura 
7.3. Analisando a tabela 7.1 é possível observar que aumentando a polaridade do meio, o comprimento de onda de máxima absorção, $\lambda_{\max }$, associado a forma neutra da MB, sofre um deslocamento para valores menores. Esse comportamento caracteriza um solvatocromismo negativo para a transição eletrônica. Para ilustrar esse comportamento, podemos observar o que ocorre com $\lambda_{\max }$ quando há uma mudança de polaridade do meio água-clorofórmio. O valor para o comprimento de onda de máxima absorção, $\lambda_{\max }$, em clorofórmio é de $615 \mathrm{~nm}$ e em água a máxima absorção ocorre em $444 \mathrm{~nm}$, sendo assim, a mudança no meio provoca um deslocamento de 171 nm no espectro de absorção da MB. Na figura 7.3, os espectros, a partir da solução estoque alcalina, mostram a existência de duas bandas para os solventes DMSO, acetonitrila e clorofórmio, a primeira na região de 650 a $550 \mathrm{~nm}$ e a segunda na região de 450 a $300 \mathrm{~nm}$. Associamos a primeira banda a forma neutra MB da sonda, devido ao fato da solução estoque ser muito alcalina e só existir a forma neutra no filme da sonda nessa condição de basicidade. Este filme foi misturado em cada solvente separadamente e específicamente em DMSO, acetonitrila e clorofórmio, esses solventes não têm força iônica capaz de promover a protonação da MB. Entretanto, a segunda banda tem $\lambda_{\max }$ equivalente a forma protonada $\mathrm{MBH}^{+}(393 \mathrm{~nm}$ para DMSO, $383 \mathrm{~nm}$ para acetonitrila e $397 \mathrm{~nm}$ em clorofórmio na figura 7.2 e $391 \mathrm{~nm}$ para DMSO, 387 $\mathrm{nm}$ para acetonitrila e $408 \mathrm{~nm}$ em clorofórmio na figura 7.3). Lembrando que a MB tem momento de dipolo alto, acreditamos que haverá alguma agregação dessa sonda em solventes de baixa polaridade, pois a interação sonda-sonda será energeticamente mais favorável que sonda-solvente de baixa polarizada. Sendo assim, acreditamos que a segunda banda pode estar associada a agregação da forma neutra de MB nestes solventes. Analisaremos a possibilidade de agregação da MB mais adiante neste capítulo. Entretanto, considerando que é possível haver a formação de dímeros de $\mathrm{MB}\left(\mathrm{MB}_{2}\right.$, ver figura 5.2 em solução de baixa polaridade, devido a formação das duas ligações de hidrogênio entre o grupo carbonila, $\mathrm{CO}$, e metila, $\mathrm{CH}_{3}$, é possível que haja reações 
de transferência de protons, $\mathrm{H}^{+}$, intradímero, produzindo assim a forma protonada $\mathrm{MBH}^{+}$ou tautomérica da $\mathrm{MB}$, onde o grupo metila transfere o proton para o grupo carbonila, que produziria a segunda banda. Essas reações de transferência de protons na $\mathrm{MB}_{2}$ em solução de baixa polaridade não foram abordadas neste trabalho, porém está sendo tema de trabalho de outro projeto de pesquisa no grupo.

Com relação a banda corresponde à forma protonada da sonda, obtida na solução estoque ácida (ver figura 7.2 e tabela 7.1 não é possível observar uma tendência definida no deslocamento solvatocrômico com o aumento na polaridade do meio, mas observamos que o efeito do solvatocromismo é menos acentuado nessa estrutura.

Como uma forma complementar na investigação do solvatocromismo das formas neutra e protonada da sonda MB, estudamos o espectro de absorção da sonda para diferentes valores de pH, não somente nas regiões extremas como apresentado anteriormente. As medidas foram realizadas em dois solventes: água e metanol. Com essa análise foi possível obter o $\mathrm{pK}_{a}$ da sonda nos dois solventes mencionados. Na seção a seguir apresentamos os resultados obtidos.

\subsection{Titulação espectroscópica e determinação de pKa}

Nessa seção apresentaremos os resultados obtidos para o estudo do espectro de absorção da sonda MB obtido em água e metanol considerando soluções com diferentes valores de pH. Desta forma, é possível observar as diferenças eletrônicas entre a forma neutra e protonada, além disso determinar o pKa da sonda em cada solvente.

O equilíbrio químico envolvendo ácidos e bases é de fundamental importância, devido a sua atuação em uma variedade de funções nos organismos vivos, como transporte de elétrons e respiração e também na construção de dispositivos eletrônicos 130. Aqui, segundo o conceito de Brönsted e Lowry [131,132, ácidos são espécies (íons ou moléculas neutras) doadoras de prótons $\left(H^{+}\right)$e bases, são espécies aceitadoras de 
prótons.

O equilíbrio químico envolvendo ácidos e bases de uma molécula qualquer $A \mathrm{em}$ solução pode ser ilustrado pela equação 7.1

$$
H A_{(s o l)}^{+} \stackrel{K_{a}}{\rightleftarrows} A_{(s o l)}+H_{(s o l)}^{+}
$$

onde a constante $K_{a}$, definida como a constante de dissociação da reação, pode ser obtida pela seguinte relação

$$
K_{a}=\frac{\left[A_{(s o l)}\right]\left[H_{(s o l)}^{+}\right]}{\left[H A_{(s o l)}^{+}\right]}
$$

Tomando o logaritmo nos dois lados da equação 7.2 , temos:

$$
\log K_{a}=\log \left[A_{(s o l)}\right]+\log \left[H_{(s o l)}^{+}\right]-\log \left[H A_{(s o l)}^{+}\right]
$$

sabendo que:

$$
p H=-\log \left[H_{(s o l)}^{+}\right]
$$

e

$$
p K_{a}=-\log K_{a}
$$

Nós podemos reescrever a equação 7.3 como

$$
p K_{a}=p H+\log \frac{\left[H A_{(s o l)}^{+}\right]}{\left[A_{(s o l)}\right]}
$$

A equação 7.6 é conhecida como equação de Henderson-Hasselbalch (HH) 133 134, nessa equação o $p K_{a}$ de um ácido está correlacionado com o pH do meio e com a concentração de suas espécies dissociadas e não dissociadas. Segundo a equação de HH, o valor de $p K_{a}$ corresponde ao valor de $\mathrm{pH}$ onde o ácido está $50 \%$ ionizado. O conhecimento do $p K_{a}$ de uma substância é muito importante para o entendimento de suas propriedades químicas, e também de grande utilidade para a previsão do comportamento da substância em diferentes condições de pH. 
Há diferentes procedimentos para a determinação experimental dos valores de $p K_{a}$, entre os métodos mais comuns podemos citar: titulação espectroscópica, condutimetria, espectroscopias Raman e ultravioleta e cromatografia líquida de alta eficiência (HPLC). Vale lembrar que o ácido e sua base conjugada devem possuir propriedades diferentes no método escolhido. Por exemplo, na espectroscopia ultravioleta a máxima absorbância das duas espécies devem ocorrer em regiões distintas.

O procedimento utilizado por nós para determinarmos o $p K_{a}$ da merocianina de Brooker foi a Titulação Espectroscópica (TE) [135, 136], em que observamos a absorbância da espécie para diferentes valores de pH. A seguir apresentamos os resultados obtidos para o espectro de absorção da sonda seguindo o procedimento de variação de pH da solução.

\subsubsection{Espectro de absorção: Água}

O espectro de absorção UV-vis da sonda MB foi medido em água variando o pH da solução no intervalo entre 2.2 e 11.8. A curva do espectro é apresentado na figura 7.4.

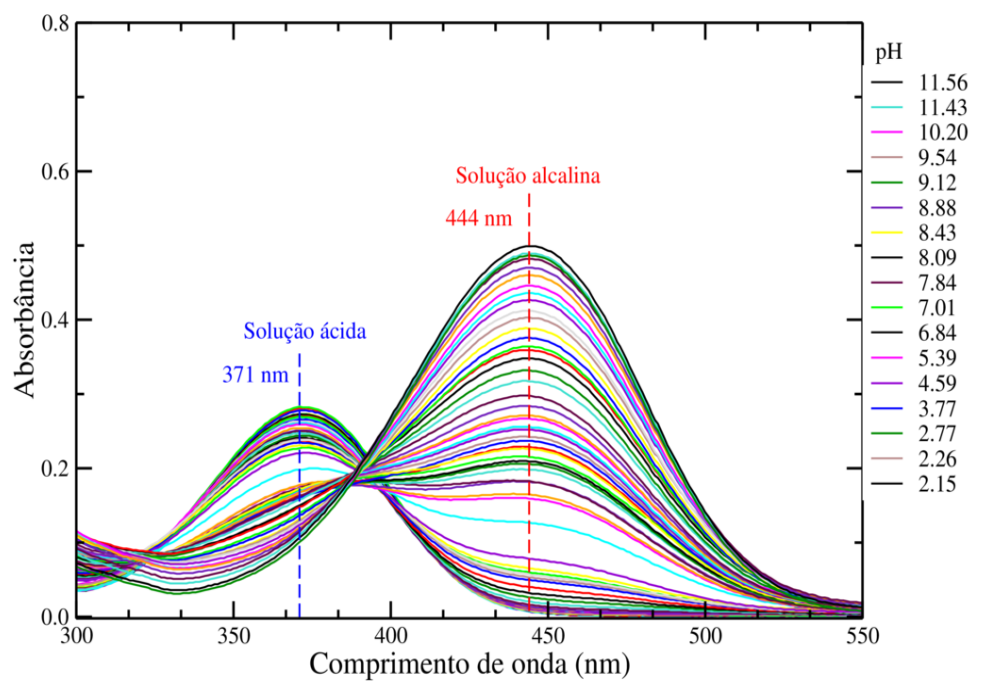

Figura 7.4: Efeito do pH sobre o espectro eletrônico de absorção da sonda MB em água à concentração de $0.015 \mathrm{mM}$. 
Observa-se na figura 7.4 que a sonda $\mathrm{MB}$ é bastante sensível à mudança de $\mathrm{pH}$ do meio. Observa-se um deslocamento no comprimento de onda de máxima absorção da sonda quando há mudança de solução ácida para solução alcalina. Observa-se na figura 7.4 que em solução ácida a máxima absorção da sonda é obtida em $371 \mathrm{~nm}$. À medida que o pH da solução se torna mais alcalino surge uma nova banda de absorção em 444 nm, o crescimento da absorbância da banda em $444 \mathrm{~nm}$ acompanha o aumento do $\mathrm{pH}$ da solução. Paralelo a esse comportamento ocorre uma diminuição na absorbância da banda em $371 \mathrm{~nm}$. Temos, portanto, duas espécies absorvedoras diferentes: a forma protonada $\mathrm{MBH}^{+}$que está presente para baixos valores de $\mathrm{pH}$ (solução ácida) e possui máxima absorbância em 371 nm, e a forma neutra MB que é predominante na solução com altos valores de pH (solução alcalina) possuindo uma máxima absorbância em $444 \mathrm{~nm}$. Vemos então que ocorre um processo de protonação/desprotonação da sonda dependente do $\mathrm{pH}$ do ambiente.

Na seção a seguir descrevemos os resultados obtidos para o estudo da sonda em metanol novamente variando o pH da solução.

\subsubsection{Espectro de absorção: Metanol}

O mesmo procedimento foi realizado para a sonda embebida em metanol. $\mathrm{Na}$ figura 7.5 apresentamos a curva de titulação espectroscópica para a sonda MB em metanol. Variamos o $\mathrm{pH}_{a p}$ da solução no intervao entre 0.0 e 12.2. Observa-se pela figura 7.5 que a sonda apresenta duas bandas distintas dependente do $\mathrm{pH}_{a p}$ na solução. Essas regiões estão separadas entre solução ácida e solução alcalina. Na solução ácida vemos um máximo de absorção no comprimento de onda igual a 390 nm. À medida que o $\mathrm{pH}_{a p}$ da solução aumenta (torna-se mais acalino) surge uma banda com máximo de absorção em $485 \mathrm{~nm}$, concomitantemente vemos uma diminuição na absorbância da banda em 390 nm e um aumento da absorbância da banda em 485 nm. Temos, portanto, duas estruturas absorvedoras diferentes: a estrutura protonada, $\mathrm{MBH}^{+}$, que 
é predominante na região ácida (baixos valores de $\mathrm{pH}$ ) e a estrutura neutra, MB, é predominante na região alcalina (altos valores de $\mathrm{pH}$ ).

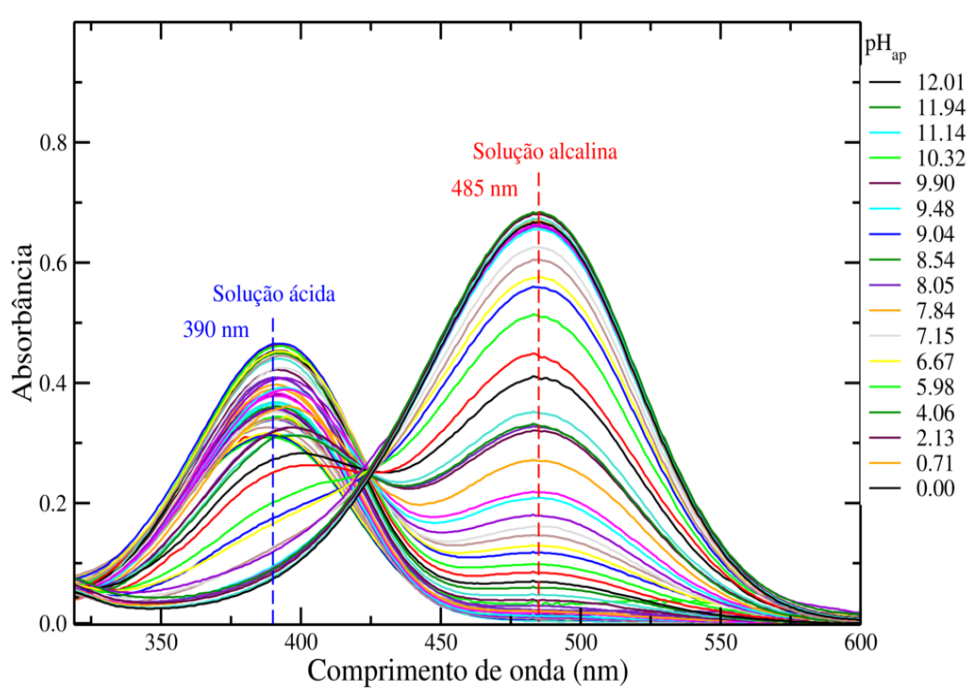

Figura 7.5: Efeito do $\mathrm{pH}_{a p}$ sobre o espectro eletrônico de absorção da sonda MB em metanol. A concentração da sonda considerada foi de $0.015 \mathrm{mM}$.

Analisando os resultados obtidos para a titulação espectroscópica tanto em água como em metanol, nós observamos que há realmente uma dependência da forma da sonda com o pH $\left(\mathrm{pH}_{a p}\right)$ da solução. Vimos que a absorção eletrônica da sonda MB apresenta duas bandas bem distintas. Em solução ácida observamos uma máxima absorbância na região entre 370 e 390 nm, e por outro lado, quando a solução está alcalina observamos que a máxima absorbância ocorre entre 444 e 485 nm, a depender do solvente considerado. Dessa forma, essa sonda pode ser utilizada também como um marcardor de pH da solução onde está inserida, devido à sua sensibilidade à concentração de íons $H^{+}$. Uma análise posterior que fizemos foi utilizar esse estudo da variação do $\mathrm{pH}\left(\mathrm{pH}_{a p}\right)$ para obtermos informações sobre parâmetros dissociativos da molécula de interesse, como por exemplo, valores de $p K_{a}$. Essa análise será apresentada na seção seguinte. 


\subsubsection{Determinação da constante de acidez}

Agora usando os resultados obtidos para o espectro de absorção da sonda em diferentes condições de $\mathrm{pH}$ nós iremos determinar os valores de $p K_{a}$ associados aos processos de protonação/desprotonação.

A probabilidade de protonação de um único sítio num soluto é dado pela equação 7.7. Algebricamente, essa equação é equivalente à equação de Henderson-Hasselbalch (HH) 7.6, descrevendo uma senoidal decrescente nas curvas de titulação padrão:

$$
\xi=\frac{10^{\left(p K_{a}-p H\right)}}{1+10^{\left(p K_{a}-p H\right)}}
$$

Assim, o valor de $p K_{a}$ para um sítio isolado titulável é igual ao $\mathrm{pH}$ numa condição em que a probabilidade de protonação desse sítio for igual a 0.5. A concentração da forma protonada $\left(\left[\mathrm{MBH}^{+}\right]\right)$e a desprotonada $([M B])$ são dada por:

$$
\left[M B H^{+}\right]=[M B]_{T} \frac{10^{\left(p K_{a}-p H\right)}}{1+10^{\left(p K_{a}-p H\right)}}
$$

$\mathrm{e}$

$$
[M B]=[M B]_{T} \frac{1}{1+10^{\left(p K_{a}-p H\right)}}
$$

onde $[M B]_{T}=\left[M B H^{+}\right]+[M B]$ é a concentração total do soluto.

Usando a técnica de titulação espectrofotométrica UV/Vis, assume-se que as formas protonada e desprotonada do soluto absorvem luz em diferentes comprimentos de onda dando origem a duas bandas diferentes no espectro UV/Vis. Considerando a Lei de Lambert-Beer [137,138], que relaciona absorbância com concentração, a absorbância total pode ser escrita pela soma individual das absorbâncias das duas formas do soluto:

$$
A \equiv \log \frac{I_{0}}{I}=\varepsilon_{0}[M B] l+\varepsilon_{1}\left[M B H^{+}\right] l
$$

onde $A$ é a absorbância medida, $I_{0}$ é a intensidade da luz incidente na amostra e $I$ é a intensidade da luz após atravessar a amostra, $\varepsilon_{0}$ e $\varepsilon_{1}$ são os coeficientes de extinção molar da forma desprotonada e protonada respectivamente e $l$ é o comprimento do 
caminho óptico. Substituindo na equação 7.10 as concentrações das formas desprotonada, $[M B]$, e protonada, $\left[M B H^{+}\right]$, em relação a concentração total, $[M B]_{T}$ (equações 7.8 e 7.9 , temos:

$$
A=A_{0} \frac{1}{1+10^{\left(p K_{a}-p H\right)}}+A_{1} \frac{10^{\left(p K_{a}-p H\right)}}{1+10^{\left(p K_{a}-p H\right)}}
$$

onde $A$ é a absorbância total medida do sistema num dado comprimento de onda. Os valores para $A_{0}, A_{1}$ e $\mathrm{pK}_{a}$ são obtidos do ajuste desta equação aos dados experimentais. Assim, monitorando a absorbância de uma amostra num comprimento de onda específico que é decrescente com o aumento do pH da solução, é possível determinarmos o valor de $\mathrm{pK}_{a}$ da amostra usando a equação 7.11. Quando a absorbância cresce com o aumento do pH da solução, o termo $\left(p K_{a}-p H\right)$ da equação 7.11 deve ser trocado de sinal, ou seja ficando $\left(p H-p K_{a}\right)$, para descrever uma senoidal crescente.

Para os dois solventes estudados, nós realizamos o ajuste dos dados experimentais considerando a equação 7.11. Foram considerados os comprimentos de onda de absorção que estão associados às duas regiões absorverdoras distintas, foram eles: 371 e $444 \mathrm{~nm}$ para solução aquosa e 390 e $485 \mathrm{~nm}$ para solução de metanol. Aqui é importante ressaltar que a nossa escolha do comprimento de onda para a determinação do $\mathrm{pK}_{a}$ corresponde aos máximos das banda das formas protonada e desprotonada, respectivamente. Para o comprimento de onda em 371 e 390 nm a absorbância é predominantemente devido à forma ácida da molécula (protonada), por outro lado, em 444 e 485 nm a absorbância é devido à forma básica da sonda (desprotonada).

Na figura 7.6 apresentamos o comportamento da sonda variando o pH da solução aquosa, bem como o ajuste obtido para os pontos experimentais. Considerando o ajuste feito em $\lambda=371 \mathrm{~nm}$, nós obtivemos um $\mathrm{pK}_{a}=8.0$ com um coeficiente de determinação $r^{2}=0.97$. O ajuste feito em $\lambda=444 \mathrm{~nm}$ nos fornece um valor $\mathrm{pK}_{a}=8.7 \mathrm{com}$ um coeficiente de determinação $r^{2}=0.98$. Consideramos o valor 8.7 (obtido do ajuste em $444 \mathrm{~nm}$ ) nosso valor mais preciso para o $p K_{a}$ da MB em solução aquosa. Isso se deve a: (i) a função ajustada ter melhor qualidade em $444 \mathrm{~nm}$ (ver valor de $r^{2}$ ); (ii) 
a banda é mais intensa em $444 \mathrm{~nm}$, o que faz com que as flutuações da absorbância seja percentualmente menores; e (iii) essa banda tem absorbância nula quando o pH é muito ácido, mostrando que a forma protonada não contribui em $444 \mathrm{~nm}$.

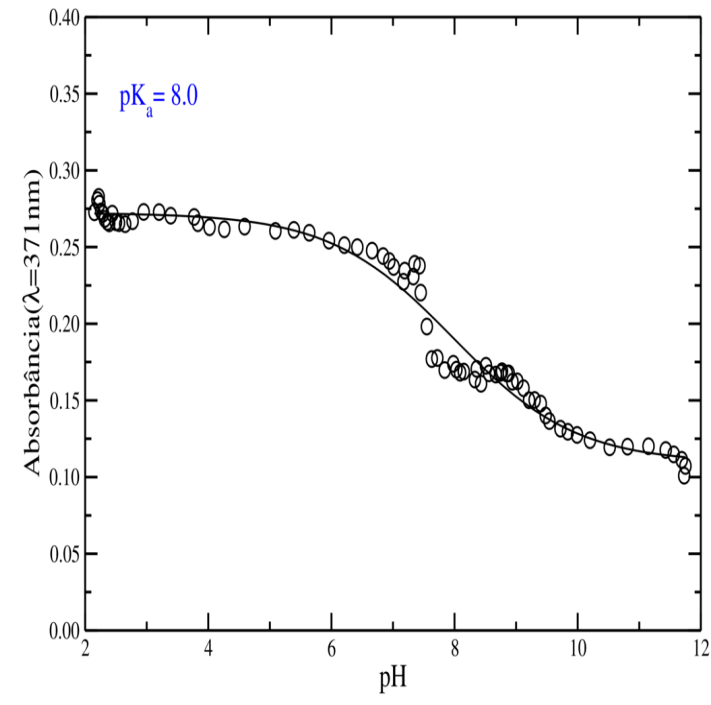

(a) $371 \mathrm{~nm}$

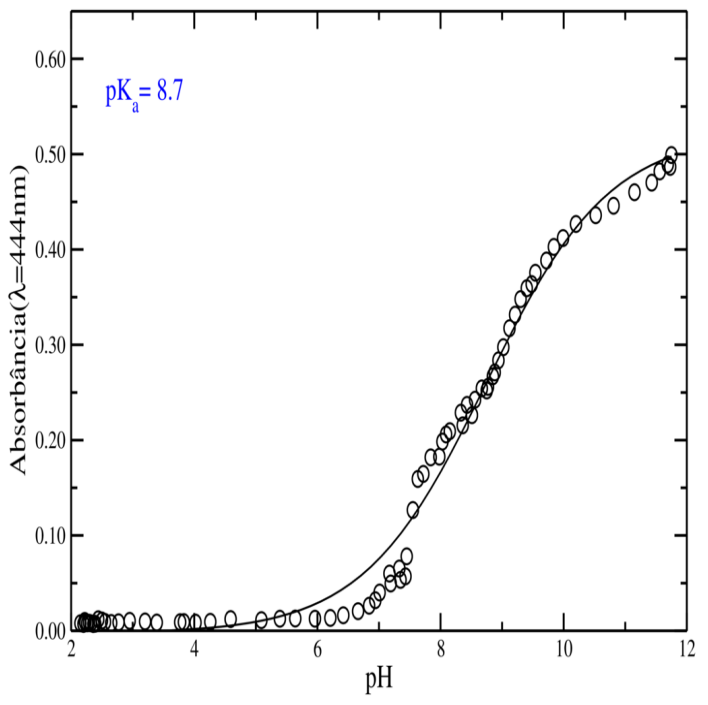

(b) $444 \mathrm{~nm}$

Figura 7.6: Comportamento da absorbância da sonda MB em água variando o pH do meio nos comprimentos de onda: (a) $371 \mathrm{~nm}$ e (b) $444 \mathrm{~nm}$. Apresentamos o ajuste dos pontos experimentais considerando a equação 7.11 .

O nosso resultado experimental de $\mathrm{pK}_{a}=8.7$ está em boa concordância com os valores existentes na literatura de $8.6[33 \mid$ e $8.4[32]$. O valor teórico que determinamos (apresentado na seção 5.3 utilizando modelo contínuo para o solvente e desprezando os efeitos de relaxação de geometria) foi de 12.4, o que apresenta a uma diferença de aproximadamente 3.7 no valor de $\mathrm{pK}_{a}$ quando comparado com o valor experimental. Essa diferença é grande ( $5 \mathrm{kcal} / \mathrm{mol}$ na energia livre de desprotonação) considerando a capacidade de predição deste modelo que é aproximadamente $2 \mathrm{kcal} / \mathrm{mol}$. Porém no caso da MB, verificamos que a solução aquosa (mesmo incluída com modelo contínuo) é capaz de promover alterações na geometria levando a sonda da forma quinoidal para uma forma intermediária, a benzoidal (zwiteriônica), e essas mudanças de geometria que foram desprezadas em nossos cálculos podem facilmente responder por essa difer- 
ença entre o $\mathrm{pK}_{a}$ teórico e experimental.

Novamente, assim como foi feito no experimento em água, nós obtivemos informação sobre o $\mathrm{pK}_{a}$ da sonda em metanol. Na figura 7.7 apresentamos o ajuste da equação 7.11 para a solução de metanol nos dois comprimentos de onda, 390 e 485 nm.

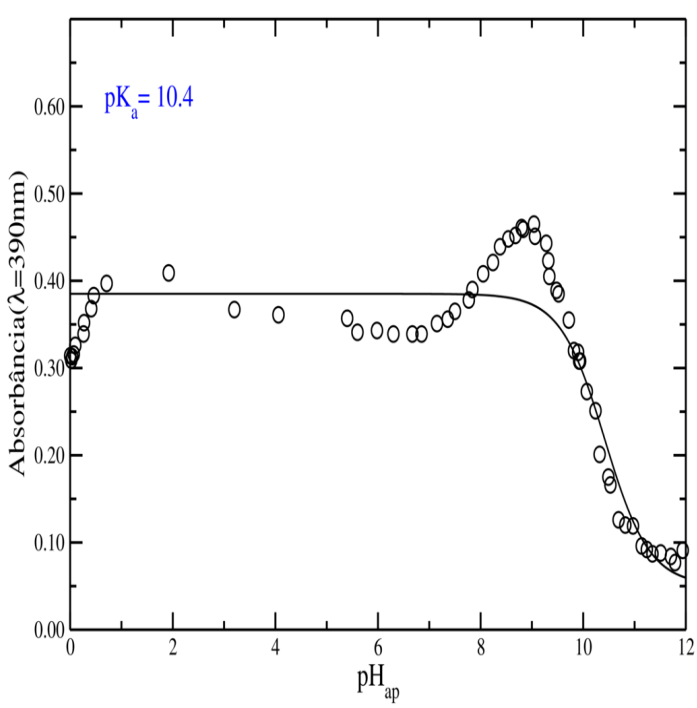

(a) $390 \mathrm{~nm}$

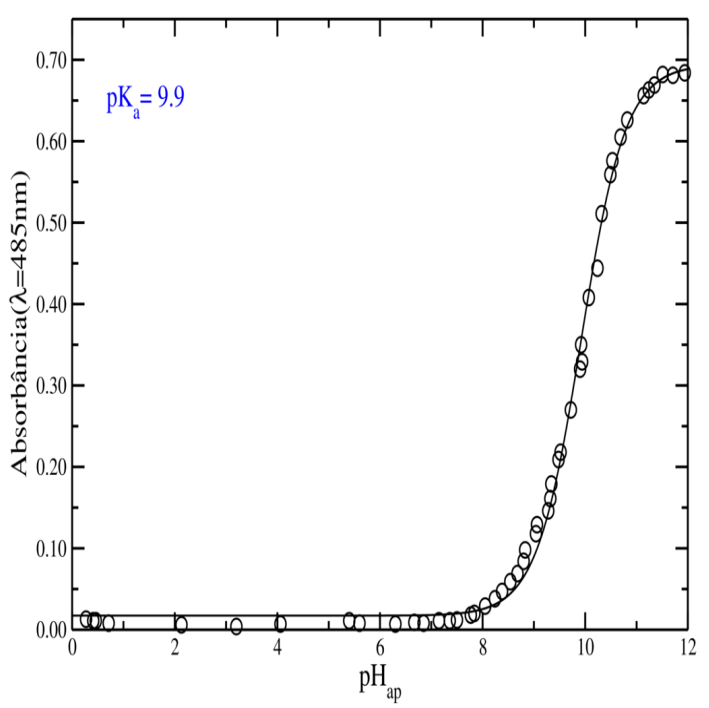

(b) $485 \mathrm{~nm}$

Figura 7.7: Comportamento da absorbância da sonda MB em metanol variando o $\mathrm{pH}_{a p}$ do meio nos comprimentos de onda: (a) $390 \mathrm{~nm}$ e (b) $485 \mathrm{~nm}$. Apresentamos o ajuste dos pontos experimentais considerando a equação 7.11 .

Aqui, considerando o ajuste feito para $\lambda=390 \mathrm{~nm}$, nós determinamos o $\mathrm{pK}_{a p}=$ 10.4 da MB em metanol. O ajuste feito para $\lambda=485 \mathrm{~nm}$, nos fornece um valor de $\mathrm{pK}_{a p}=9.9$. Consideramos o valor 9.9 (obtido do ajuste em $485 \mathrm{~nm}$ ) nosso valor mais preciso para o $p K_{a}$ da $\mathrm{MB}$ em solução de metanol. Isso se deve aos mesmos motivos apresentado anteriormente para a solução aquosa. Na literatura não há outros trabalhos em que é investigado o processo de protonação/desprotonação da sonda MB em metanol. Podemos comparar esse nosso valor experimental com o cálculo teórico para o $\mathrm{pK}_{a p}$ apresentado na seção 5.3, o valor teórico obtido foi de $\mathrm{pK}_{a p}=13.5$, o que representa uma diferença de 3.6 no valor do $\mathrm{pK}_{a p}$ comparativamente ao dado 
experimental. Note que essa diferença entre o valor teórico e experimental do $\mathrm{pK}_{a p}$ é da mesma magnitude daquele encontrado para a solução aquosa e isso corrobora nossa expectativa que essa diferença é devido às mudanças geométricas da $\mathrm{MB}$ em vácuo e em solução não consideradas no cálculo teórico.

Um passo seguinte no nosso estudo foi investigar se há formação de agregados da sonda em alguns solventes: água, metanol e acetonitrila. Esse estudo será detalhado a seguir.

\subsection{Estudo da agregação da sonda MB}

Nesta seção faremos uma análise da agregação da sonda em solventes com polaridades diferentes: água, metanol e acetonitrila. O estudo foi realizado variando a concentração do soluto tipicamente em valores entre $0.006 \mathrm{mM}$ até $0.100 \mathrm{mM}$. Aqui foi levado em conta também ambientes com diferentes valores de $\mathrm{pH}$ aparente $\left(\mathrm{pH}_{a p}\right)$.

\section{Solvente: água}

O estudo do processo de agregação da sonda em água foi realizado em solução alcalina $(\mathrm{pH}=13.5)$. Essa condição nos garante que na solução teremos somente uma única estrutura absorvedora, a forma desprotonada/neutra MB. Variamos a concentração do soluto no intervalo de $0.001 \mathrm{mM}$ até $0.060 \mathrm{mM}$. Os resultados obtidos para a absorbância em diferentes valores de concentração estão apresentados na figura 7.8 . 


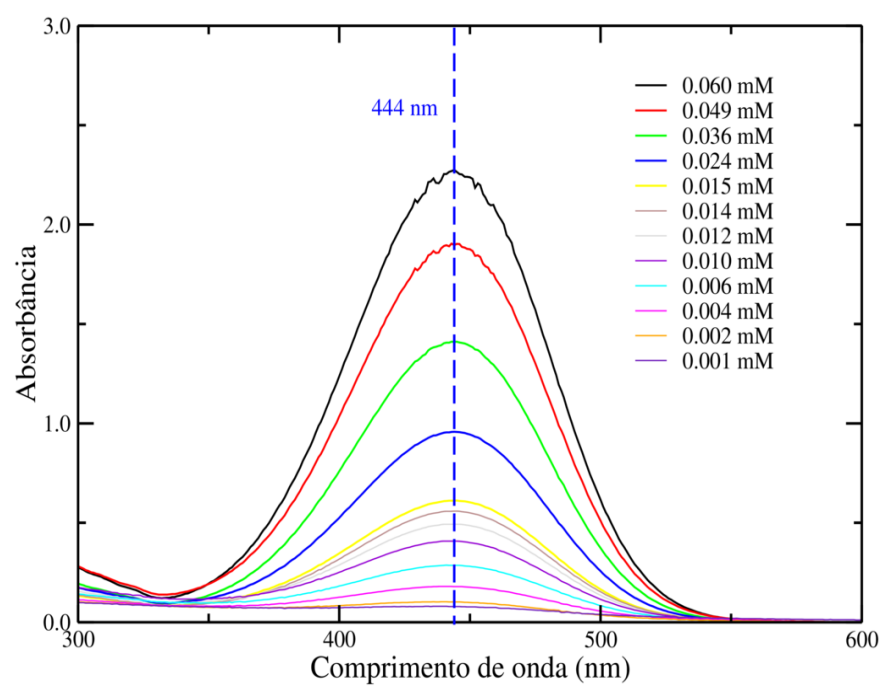

Figura 7.8: Espectro eletrônico de absorção da sonda MB em solução aquosa variando a concentração do soluto. Solução alcalina, $\mathrm{pH}=13.5$.

Podemos observar na figura 7.8 que a banda de $444 \mathrm{~nm}$ reduz sua intensidade com a redução da concentração do soluto, mas não identificamos deslocamentos na banda. Analisamos também o comportamento da absorbância versus a concentracão do soluto para verificar a validade da Lei de Lambert-Beer, que assume a hipótese de não agregação do soluto e descreve um comportamento linear da absorbância com a concentração do soluto. O resultado para esta análise está apresentado na figura 7.9 .

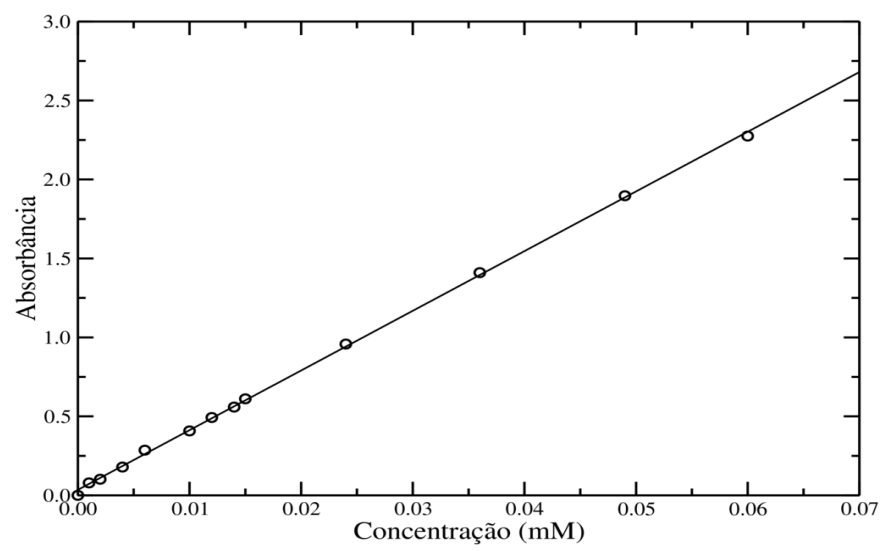

Figura 7.9: Comportamento da absorbância da sonda MB em solução aquosa variando a concentração do soluto. Solução alcalina, $\mathrm{pH}=13.5$. 
Observa-se que a Lei de Bouguer-Lambert-Beer é válida e há uma relação linear entre a absorbância e a concentração. Esse resultado confirma que não há autoagregação da sonda MB na forma neutra em solução aquosa. Esse resultado está em concordância com nossas observações nas simulações computacionais que mostram a separação do dímero antiparalelo da MB em água. Essa separação ocorre rapidamente, com apenas 100 mil ciclos MC da simulação. Durante um tempo muito logo de simulação, 1 milhão de ciclos MC, as duas moléculas de MB ficam separadas e inteiramente solvatadas em água.

\subsubsection{Solvente: metanol}

O estudo do processo de agregação da sonda em metanol foi realizado em duas condições distintas: solução ácida $\left(\mathrm{pH}_{a p}=0.3\right)$ e solução alcalina $\left(\mathrm{pH}_{a p}=12.6\right)$. Essas condições nos garante que em cada solução teremos predominantemente uma única estrutura absorvedora. Assim, em $\mathrm{pH}_{a p}=0.3$ a forma predominante será a protonada/catiônica, $\mathrm{MBH}^{+}$. Em solução $\mathrm{com} \mathrm{pH}_{a p}=12.6$ a forma absorvedora será a forma desprotonada/neutra, MB. Estudamos o comportamento da absorbância da sonda para diferentes valores de concentração.

\section{Solução ácida, $\mathrm{pH}_{a p}=0.3$}

Medimos o espectro de absorção da sonda para diferentes valores de concentração, variando de $0.006 \mathrm{mM}$ até $0.100 \mathrm{mM}$. Observamos pela figura 7.10 que o aumento da concentração do soluto aumenta a absorbância do sistema, porém não provoca nenhum deslocamento na banda ou surgimento de novas bandas, o que nos indica que a substância absorvedora continua a mesma e não há formação de agregados nessa faixa de concentração estudado. Fazendo a normalização desses espectros pelo máximo da absorbância (ver também na figura 7.10), observamos que a variação da concentração não provoca uma mudança na forma do espectro. 


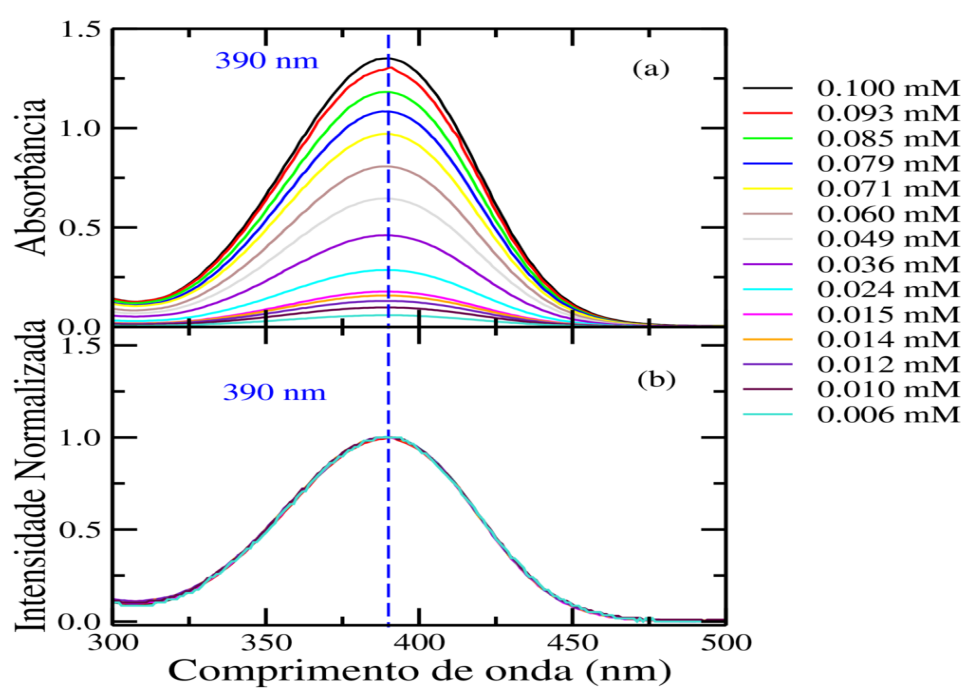

Figura 7.10: Espectro eletrônico de absorção da sonda MB em solução de metanol variando a concentração do soluto. Solução ácida, $\mathrm{pH}_{a p}=0.3$. Absorbâncias medidas (a) e normalizada (b).

Analisamos também o comportamento da absorbância versus a concentracão do soluto. O resultado para esta análise está apresentado na figura 7.11. Observa-se que a Lei de Lambert-Beer é válida e há uma relação linear entre a absorbância e a concentração. Esse resultado confirma que não há autoagregação da sonda na forma protonada/catiônica $\mathrm{MBH}^{+}$em solução de metanol. Esse resultado é esperado para solutos carregados em solventes polares, pois a interação entre os solutos com mesma carga é repulsiva, enquanto que a interação soluto carregado-solvente é atrativa. 


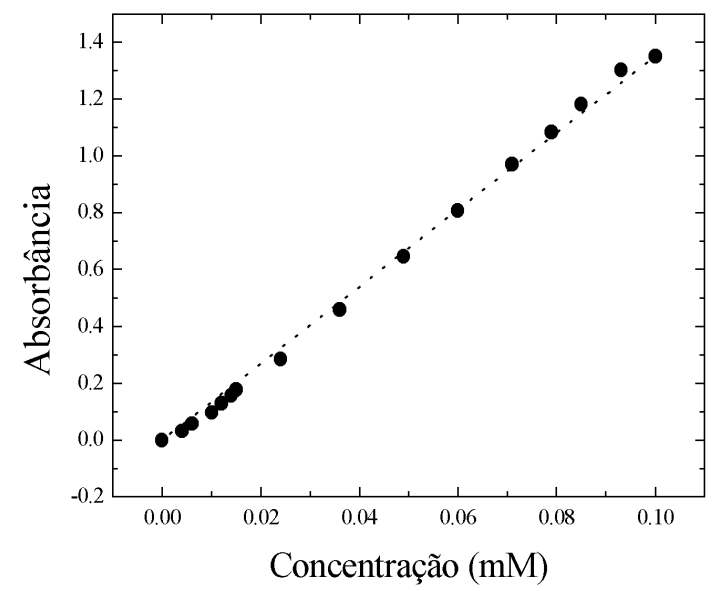

Figura 7.11: Comportamento da absorbância da sonda MB em solução de metanol variando a concentração do soluto. Solução ácida, $p H_{a p}=0.3$.

\section{Solução alcalina, $\mathrm{pH}_{a p}=\mathbf{1 2 . 6}$}

Medimos o espectro de absorção da sonda para diferentes valores de concentração, variando de $0.006 \mathrm{mM}$ até $0.060 \mathrm{mM}$. Observamos pela figura 7.12 que, de forma análoga ao pH ácido, o aumento da concentração do soluto aumenta a absorbância do sistema, não existe deslocamentos, nem surgimento de novas bandas. Fazendo a normalização desses espectros pelo máximo da absorbância (ver também na figura 7.12), observamos que a variação da concentração não provoca uma mudança na forma do espectro.

Analisamos também o comportamento da absorbância versus a concentracão do soluto nessas soluções alcalinas. O resultado para esta análise está apresentado na figura 7.13. Observa-se o mesmo comportamento das soluções ácidas, ou seja, a Lei de Lambert-Beer é válida e há uma relação linear entre a absorbância e a concentração. Esse resultado confirma que não há autoagregação da sonda na forma desprotonada/neutra MB em solução de metanol. Esse resultado está em aparente disconcordância com nossas observações nas simulações computacionais que mostram 


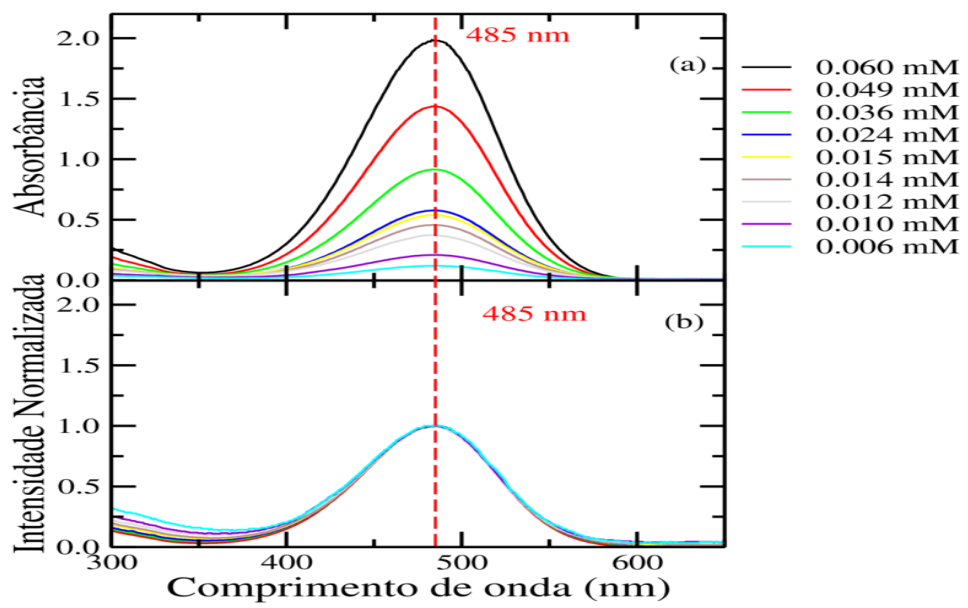

Figura 7.12: Espectro eletrônico de absorção da sonda MB em solução de metanol variando a concentração do soluto. Solução alcalina, $p H_{a p}=12.6$. Absorbâncias medidas (a) e normalizadas (b).

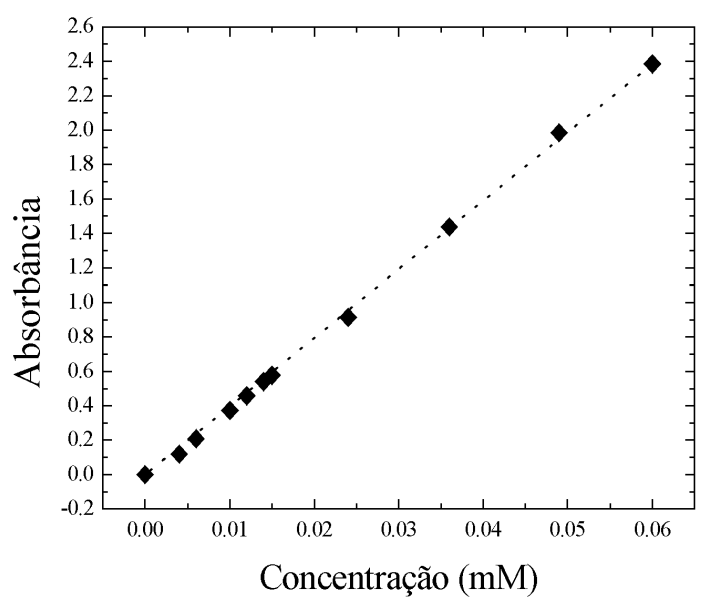

Figura 7.13: Comportamento da absorbância da sonda MB em solução de metanol variando a concentração do soluto. Solução alcalina, $\mathrm{pH}_{a p}=12.6$. 
que o dímero antiparalelo $\mathrm{MB}_{2}$ em metanol se mantém estável durante um tempo muito logo de simulação, 1 milhão de ciclos MC, (ver figura 5.9). Porém nossos valores calculados para transição eletrônica intensa do monômero $\mathrm{MB}$ e dímero $\mathrm{MB}_{2}$ em metanol mostram que ambos sistemas, $\mathrm{MB}$ e $\mathrm{MB}_{2}$, absorvem na mesma região, $442 \mathrm{e}$ $440 \mathrm{~nm}$, respectivamente, usando o modelo explícito do solvente com ASEC em nível TD-B3LYP (ver tabelas 5.11 e 5.15). Sendo assim, a banda medida experimentalmente para a sonda em metanol pode ser atribuida tanto ao monômero MB como ao dímero $\mathrm{MB}_{2}$ e caso os dímeros não se agrupem em agregados maiores com o aumento da concentração da sonda em solução, a Lei de Lambert-Beer continua válida, pois o centro absorvedor de luz seria o dímero independentemente da concentração. Desta forma, teríamos concordância entre os dados experimentias e teóricos. É importante ressaltar aqui, que os resultados teóricos mostram que o dímero $\mathrm{MB}_{2}$ em metanol também apresenta uma transição eletrônica em 619 nm, porém com baixíssima intensidade. Entretanto não sabemos se essa baixa intensidade é correta ou artificialmente obtida devido a nossas simulações serem com moléculas rígidas, pois sistemas com alta simetria (como é o caso do dímero estudado) tendem a apresentar algumas transições eletrônicas com baixa intensidade. Quando essa simetria é quebrada devido às deformações intramoleculares essas transições ganham intensidade. Em nosso caso, a dificuldade de realizar simulações com a sonda flexível recai na falta de parametrização de campo de força clássico para essa molécula e a existência de um acoplamento forte entre a estrutura eletrônica e a geometria da sonda para as formas quinoidal e benzoidal. Esse acoplamento dificulta muito a parametrização de campos de força clássico. O ideal é utilizar métodos híbridos, onde a sonda seja tratada com mecânica quântica e o solvente com mecânica clássica. Porém o tempo computacional será bastante grande devido ao tamanho da sonda $\left(\mathrm{MB}\right.$ e $\left.\mathrm{MB}_{2}\right)$. 


\subsubsection{Solvente: acetonitrila}

O estudo do processo de agregação da sonda em acetonitrila foi realizado em solução alcalina $\left(\mathrm{pH}_{a p}=11.0\right)$. Esta solução alcalina de acetonitrila foi obtida a partir da mistura de três componentes: do filme da merocianina secado da solução estoque pura, do solvente de acetonitrila e de $\mathrm{NaOH}$ na concentração de $17 \mathrm{mM}$. Na figura 7.14 apresentamos os espectros medidos para as diferentes concentrações da sonda. Nesta figura, duas bandas são claramente identificadas, uma em $564 \mathrm{~nm}$ e outra em $382 \mathrm{~nm}$. De forma análoga aos outros solventes, água e metanol, a absorbância das bandas cresce com o aumento da concentração do soluto, não há deslocamentos das duas bandas e nem aparecimentos de novas bandas. Porém analisando as curvas normalizadas pelas absorbâncias da banda em $382 \mathrm{~nm}$ (ver também na figura 7.14) observamos que existe uma mudança na forma do espectro, ou seja, a banda em 564 nm não cresce com a mesma proporção que a banda em $382 \mathrm{~nm}$.

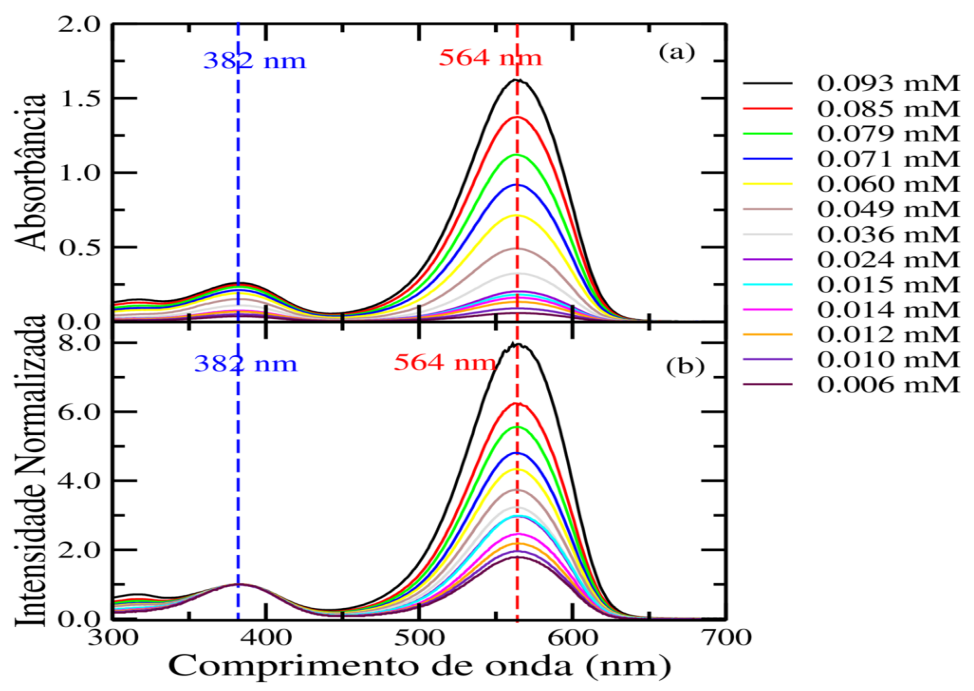

Figura 7.14: Comportamento da absorbância da sonda MB em solução de acetonitrila variando a concentração do soluto. Solução alcalina, $\mathrm{pH}_{a p}=11.0$. Absorbâncias medidas (a) e normalizadas (b).

Analisamos a curva para a absorbância versus a concentração para as duas bandas, em 382 e $564 \mathrm{~nm}$. Os resultados estão apresentados na figura 7.14. Observamos 
que a relação entre a absorbância e a concentração é quase linear. Note que todos os pontos para a banda em 382 e $564 \mathrm{~nm}$ ficam sempre acima da reta tracejada e sempre abaixo da outra reta tracejada, respectivamente. Porém a variação é pequena e não nos dá uma informação convincente sobre a aplicabilidade para este sistema, ou não, da Lei de Lambert-Beer. Nesse caso, seriam necessárias algumas repetições dessas medidas para termos um resultado mais confiável como uma média sobre várias amostras. Entretanto, identificamos claramente que as inclinações das retas para as duas bandas são distintas. Isso mostra que temos dois centros aborvedores de luz na amostra. Poderíamos interpretar esses dois absorvadores com base em duas possibilidades que na solução existem: (i) as formas desprotonada/neutra MB e protonada/catiônica $\mathrm{MBH}^{+}$; ou (ii) as formas monomérica $\mathrm{MB}$ (ou dímero $\mathrm{MB}_{2}$, ver discussão no final da subseção anterior) e agregada $\mathrm{MB}_{n}$.

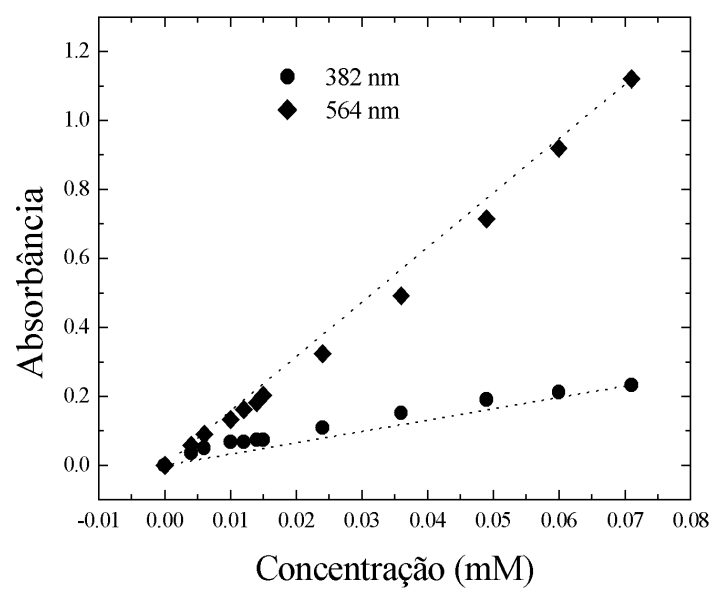

Figura 7.15: Comportamento da absorbância da sonda MB em solução de acetonitrila versus a concentração do soluto. Absorbâncias obtidas para as duas bandas, em 382 e $564 \mathrm{~nm}$.

Para investigar essas duas possibilidades apresentamos na figura 7.16 o espectro de absorção para a sonda em acetonitrila considerando amostras retiradas das duas soluções estoques: ácida, $\mathrm{pH}_{a p}=0.0$, e alcalina, $\mathrm{pH}_{a p}=13.3$. Na amostra em acetonitrila obtida a partir da solução estoque ácida, a sonda apresenta $\lambda_{\max }$ em $383 \mathrm{~nm}$. 
Essa absorção é devido à forma protonada $\mathrm{MBH}^{+}$, que está presente nesta condição de acidez. Na amostra em acetonitrila obtida a partir da solução estoque alcalina, temos a molécula na sua forma neutra MB e o seu espectro apresenta duas bandas bem definidas, a primeira com $\lambda_{\max }$ em $385 \mathrm{~nm}$ e a segunda em $568 \mathrm{~nm}$. Como a solução estoque alcalina foi preparada para termos apenas uma espécie da sonda MB, o surgimento da banda em $385 \mathrm{~nm}$ nos fez investigar a possibilidade de agregação através de duas análises. Na primeira, investigamos o comportamento as duas bandas da MB (a partir da solução estoque alcalina) com a variação da concentração. Consideramos dois valores de concentração: $15 \mu \mathrm{M}$ e $7 \mu \mathrm{M}$. O espectro medido para essas duas concentrações está apresentado na figura 7.17 .

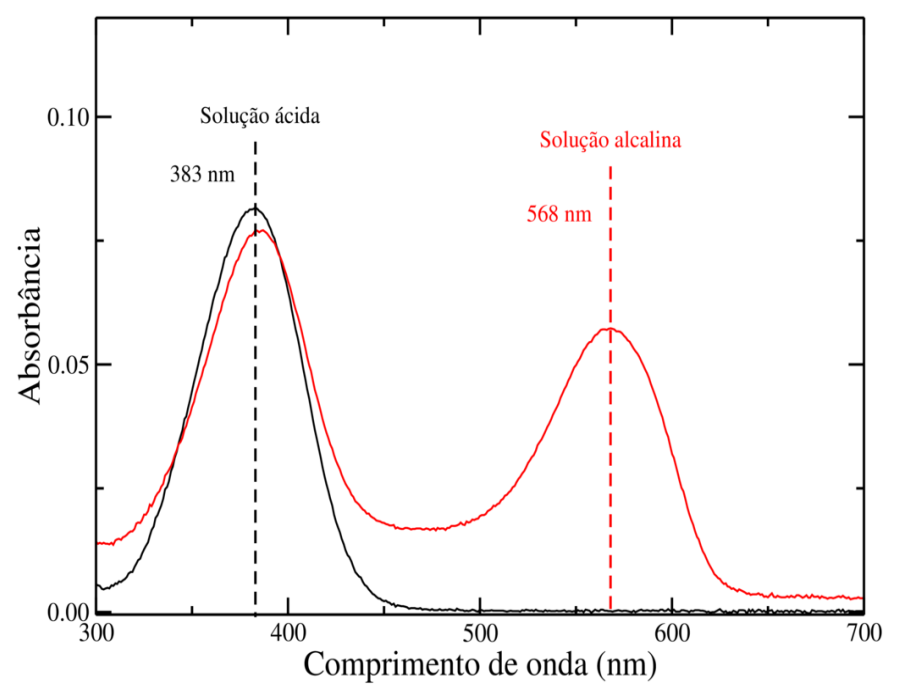

Figura 7.16: Espectro eletrônico de absorção da sonda MB em acetonitrila para duas soluções estoques diferentes: ácida e alcalina. 


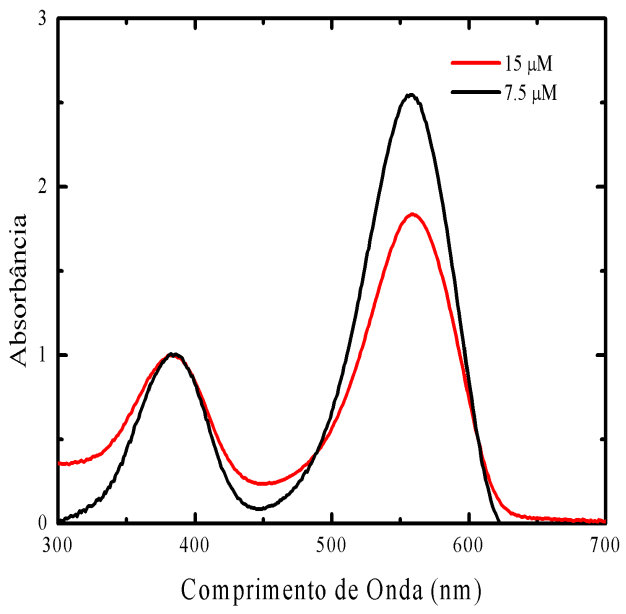

(a)

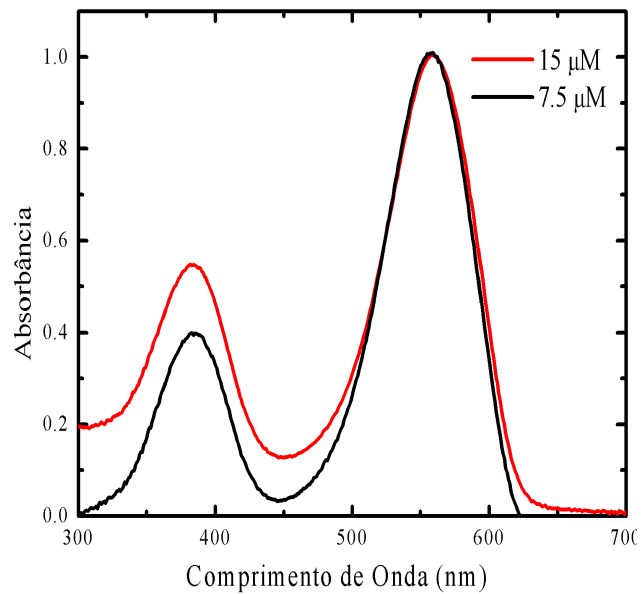

(b)

Figura 7.17: Espectro eletrônico de absorção da sonda MB em acetonitrila para duas concentrações diferentes. Solução estoque alcalina.(a) absorbância normalizada para a banda em $385 \mathrm{~nm}$, (b) absorbância normalizada para a banda em $568 \mathrm{~nm}$.

Observa-se na figura 7.17 que as duas bandas apresentam comportamento distinto com a variação da concentração.

Nós estávamos interessados em analisar a existência, ou não, da autoagregação da sonda MB em acetonitrila. Para fazer essa análise podemos variar a concentração de MB em acetonitrila e observarmos o comportamento da absorbância para cada concentração, da mesma forma que foi feito nos casos anteriores. Outra possibilidade é partir de uma dada concentração, obter o espectro eletrônico de absorção no solvente e verificar se a banda de absorção é referente a agregados. Esse foi o procedimento utilizado para a sonda MB (solução estoque alcalina) em acetonitrila. Um filme contendo MB foi diluído num tubo de ensaio juntamente com acetonitrila. Essa amostra foi colocada em banho submetido a ultrassom de $40 \mathrm{kHz}$ durante $30 \mathrm{~min}$. Em seguida, a amostra foi colocada no espectrômetro e vários espectros de absorção foram medidos em diferentes tempos: $0 \mathrm{~min}, 2 \mathrm{~h}, 2 \mathrm{~h}$ e $30 \mathrm{~min}, 3 \mathrm{~h}$ e 5 dias. Esses espectros estão apresentados na figura 7.18 . 


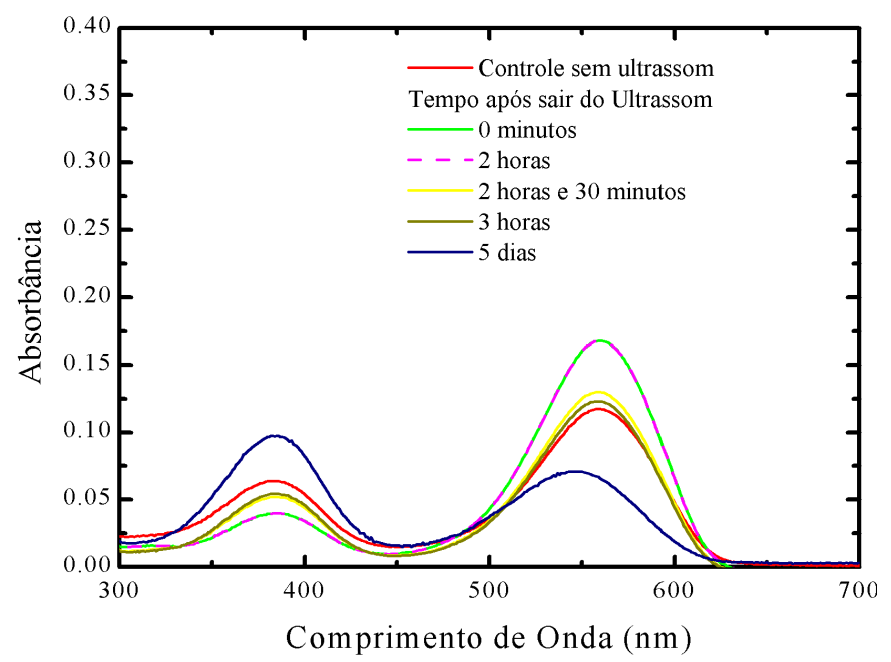

Figura 7.18: Espectro eletrônico de absorção da sonda MB em acetonitrila (amostra obtida a partir da solução estoque alcalina) antes e depois do ultrasom em diferentes intervalos de tempos.

Observamos na figura 7.18 que antes do ultrassom (linha vermelha) a primeira banda com $\lambda_{\max }$ em $568 \mathrm{~nm}$ é mais intensa que a segunda banda com $\lambda_{\max } \mathrm{em} 383 \mathrm{~nm}$. Após os 30 min de ultrassom (linha verde) verificamos um aumento de absorbância da primeira banda e uma diminuição da segunda. O calor gerado pelo ultrassom e absorvido pela amostra é capaz de desestabilizar agregados maiores. Por isso, podemos associar a segunda banda a agregados maiores da MB. A amostra medida logo após sair do ultrassom (0h) e $2 \mathrm{~h}$ após tem exatamente o mesmo espectro (linhas verde tracejada e rosa). Isso mostra que o processo de agregação é lento e é necessário esperar mais de $2 \mathrm{~h}$ após a preparação da amostra para começar a ver uma diminuição da absorbância da primeira banda. Porém, a partir desse tempo a primeira banda começa a reduzir a absorbância e a segunda começa a aumentar (linhas amarela e marrom), chegando muito próximo do espectro medido antes do ultrassom. Isso mostra que os agregados maiores voltam a ser formados. Porém esperando 5 dias, observamos que os agregados maiores continuam sendo formados e a segunda banda continua aumentando de intensidade, chegando a ficar mais intensa que a primeira banda. Isso 
nos leva a um questionamento de quanto tempo será necessário para estabilizar o espectro da amostra e se a primeira banda (com $\lambda_{\max }$ em $\left.383 \mathrm{~nm}\right)$ pode ser extinta, ou se, existe uma absorbância mínima estabilizada.

Vimos que a solução estoque alcalina da MB apresenta duas bandas no espectro de absorção quando medido em solventes de baixa polaridade como DMSO, acetonitrila e clorofórmio. Na seção a seguir apresentamos uma proposta para esse entendimento.

\subsection{Nossa proposta: transferência de prótons}

Como vimos nos gráficos 7.3 e 7.16, o espectro de absorção da MB, considerando um filme da solução estoque alcalina, em solventes de baixa polaridade considerados (DMSO, acetonitrila e clorofórmio), apresenta duas bandas distintas no espectro: uma banda com maior comprimento de onda, na região entre 550-600 nm, e uma segunda banda na região 350-400 nm. A banda de mais baixa energia temos associado à espécie de MB neutra. Por outro lado, a banda de mais alta energia apresenta uma máxima absorbância numa região muito próxima da observada para a forma protonada $\mathrm{MBH}^{+}$. Na tabela 7.2 apresentamos um comparativo entre as duas bandas: a banda da forma protonada $\left(\mathrm{MBH}^{+}\right)$e a segunda banda observada para a forma neutra MB.

Tabela 7.2: Comparativo entre o comprimento de onda de máxima absorção, $\lambda_{\max }(\mathrm{nm})$, entre a banda da solução ácida e a segunda banda observada na solução alcalina.

\begin{tabular}{ccc}
\hline \hline Solvente & Solução estoque ácida & Solução estoque alcalina $\left(2^{\circ}\right.$ banda $)$ \\
\hline & $\lambda_{\max }\left(\mathrm{MBH}^{+}\right)$ & $\lambda_{\max }(\mathrm{MB})$ \\
Acetonitrila & 383 & 387 \\
DMSO & 393 & 391 \\
Clorofórmio & 397 & 408 \\
\hline \hline
\end{tabular}


Podemos observar na tabela 7.2 que as duas bandas são realmente muito aparentes. O que nos faz pensar na possibilidade de que a segunda banda observada na solução alcalina pode ser referente à espécie protonada da MB. Porém, na nossa solução estoque há apenas população da forma neutra, MB, e os solventes de baixa polaridade considerados são todos apróticos. Portanto, quem doaria o próton para a sonda? Na nossa proposta para o entendimento desse comportamento, nós acreditamos que a MB agrega em solventes de baixa polaridade, pois ela possui um grande momento de dipolo (nós observamos experimentalmente que ela não é solúvel em benzeno e ciclo-hexano, e pouco solúvel em clorofórmio), por outro lado, nós vimos através do modelo teórico que a formação do dímero antiparalelo (dipolo nulo) favoreceria a sua solubilidade em solventes de baixa polaridade. Com o dímero formado, o próton, $\mathrm{H}^{+}$, poderá vir de um dos monômeros de acordo com as seguintes possibilidades:

- A transferência simultânea de 2 prótons gerando a forma tautomérica da MB, $\mathrm{MB}_{t}$. Ver figura 7.19. Nós calculamos a excitação eletrônica para esse estrutura isolada com B3LYP $/ 6-311+\mathrm{G}^{* *}$ e obtivemos o valor de $442 \mathrm{~nm}$. Essa transferência de próton não explica a existência das duas bandas experimentais observadas em solventes de baixa polaridade. 

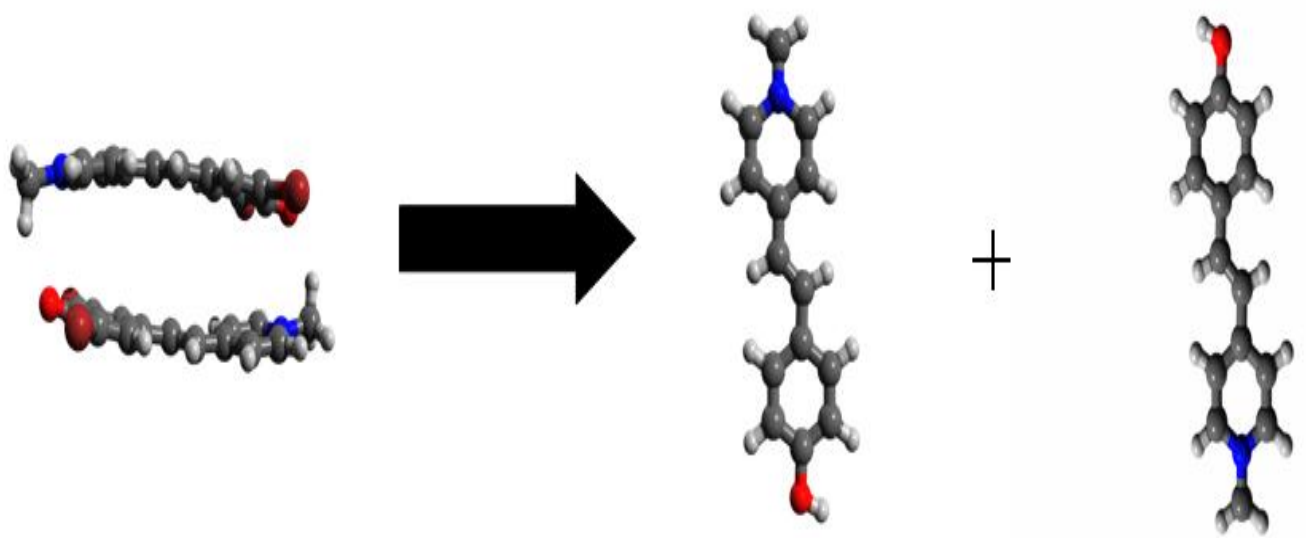

Figura 7.19: Ilustração da transferência de próton intradímeros gerando a forma tautomérica da $\mathrm{MB}, \mathrm{MB}_{t}$.

- A outra possibilidade é a transferência de 1 próton gerando a forma desprotonada $\mathrm{MB}^{-}$e a forma protonada $\mathrm{MBH}^{+}$, ver figura 7.20 . Nós calculamos a excitação eletrônica dessas formas isoladas com B3LYP $/ 6-311+\mathrm{G}^{* *}$ e obtivemos os seguintes resultados: $576 \mathrm{~nm}$ para a forma $\mathrm{MB}^{-}$e $395 \mathrm{~nm}$ para a forma $\mathrm{MBH}^{+}$. Essa proposta descreve as duas bandas observadas experimentalmente em solventes de baixa polaridade.
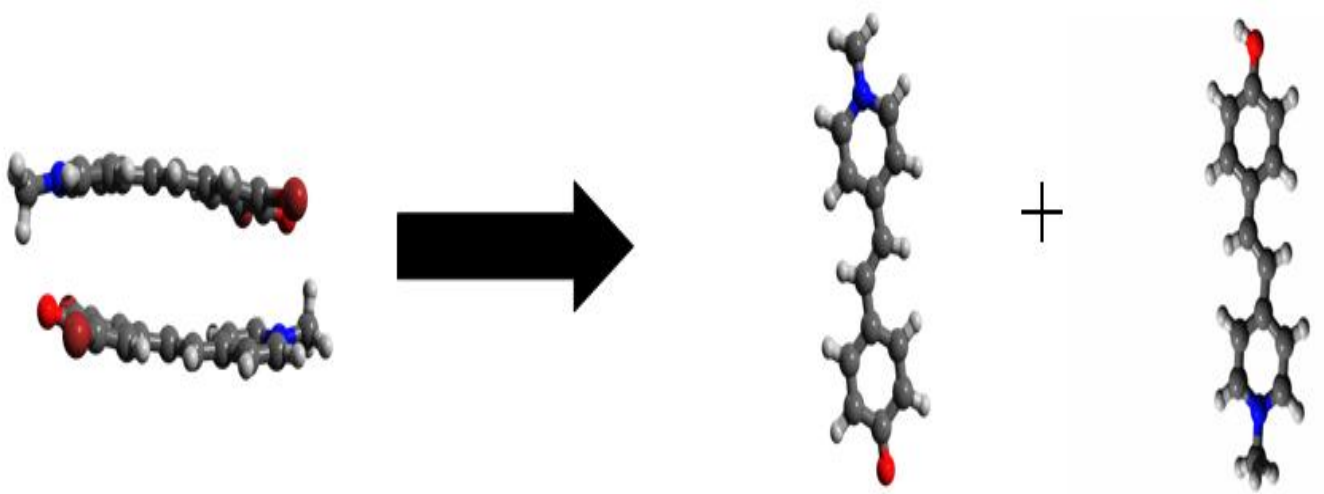

Figura 7.20: Ilustração da transferência de próton intradímeros gerando as formas $\mathrm{MB}^{-} \mathrm{e}$ $\mathrm{MBH}^{+}$. 


\section{Capítulo 8}

\section{Conclusões}

Vamos agora enunciar as principais conclusões obtidas na realização deste trabalho:

- Concluimos que a mudança estrutural cis-trans na sonda MB não pode ser observada através da análise UV-Vis, pois nossos resultados teóricos mostram que as duas estruturas absorvem na mesma região. Logo, não é esse efeito que provoca o comportamento anômalo observado no espectro das merocianinas;

- O nosso valor medido para o $\mathrm{pK}_{a}$ através da titulação espectroscópica da MB em água, 8.7, está em boa concordância com outros valores existentes na literatura, 8.6 e 8.4;

- Determinamos um valor inédito para o $\mathrm{pK}_{a p}$ da MB em metanol em 9.9;

- Os nossos valores para o $\mathrm{pK}_{a}$ teórico da MB em água e metanol superestimam o dado experimental em aproximadamente 4 unidades de $\mathrm{pK}_{a}$. Essa diferença pode ser devido a não inclusão da relaxação da geometria no respectivo solvente;

- Os espectros medidos a partir da solução estoque ácida mostram que a forma protonada, $\mathrm{MBH}^{+}$, é pouco sensível à mudança na polaridade do solvente, com deslocamentos na ordem de $20 \mathrm{~nm}$ quando ocorre a mudança água-clorofórmio. Con- 
seguimos uma boa descrição teórica para esse comportamento usando o método TD-DFT com os funcionais B3LYP e CAM-B3LYP e o conjunto de funções base $6-311+\mathrm{G}^{* *}$;

- Os espectros medidos a partir da solução estoque alcalina mostram que a forma MB é bastante sensível à mudança na polaridade do meio, apresentando deslocamentos na ordem $170 \mathrm{~nm}$ provocado pela mudança água-clorofórmio. Esse resultado está em acordo com os resultados apresentados na literatura;

- Conseguimos uma boa descrição teórica para o deslocamento solvatocrômico observado experimentalmente para a forma MB em solventes de alta polaridade, por outro lado, em solventes de baixa polaridade não conseguimos calcular a excitação eletrônica na região experimental de 550-650 nm, nesse estudo foi feito o cálculo com diversos funcionais de densidade;

- Variando a concentração da MB e analisando a absorbância do comprimento de onda de máxima absorção, verificamos a lei de Lambert-Beer, portanto, concluimos que a sonda não agrega em água. As nossas simulações computacionais corroboram esse resultado mostrando que o dímero de MB se separa em água;

- Fazendo a mesma análise para solventes de baixa polaridade (DMSO, acetonitrila e clorofórmio) identificamos dois centros distintos absorvedores de luz, pois analisando a absorbância das 2 primeiras bandas identificamos duas inclinações distintas;

- Apresentamos uma proposta de formação de dímeros que levaria a uma transferência de prótons intradímeros originando duas novas estruturas: $\mathrm{MB}^{-} \mathrm{e} \mathrm{MBH}^{+}$. A estrutura $\mathrm{MB}^{-}$apresenta a excitação eletrônica na região de 550-650 nm, que é a mesma calculada para os dímeros de MB. Essa proposta descreve a observação experimental para as duas bandas em solventes de baixa polaridade; 
- Na nossa proposta, o comportamento anômalo observado para a MB deve-se a existência de duas estruturas diferentes dependente da polaridade do meio: a forma MB descreve a solvatação em solventes de alta polaridade e a forma $\mathrm{MB}^{-}$descreve a solvatação em solventes com baixa polaridade. Na figura 8.1 ilustramos esse comportamento.

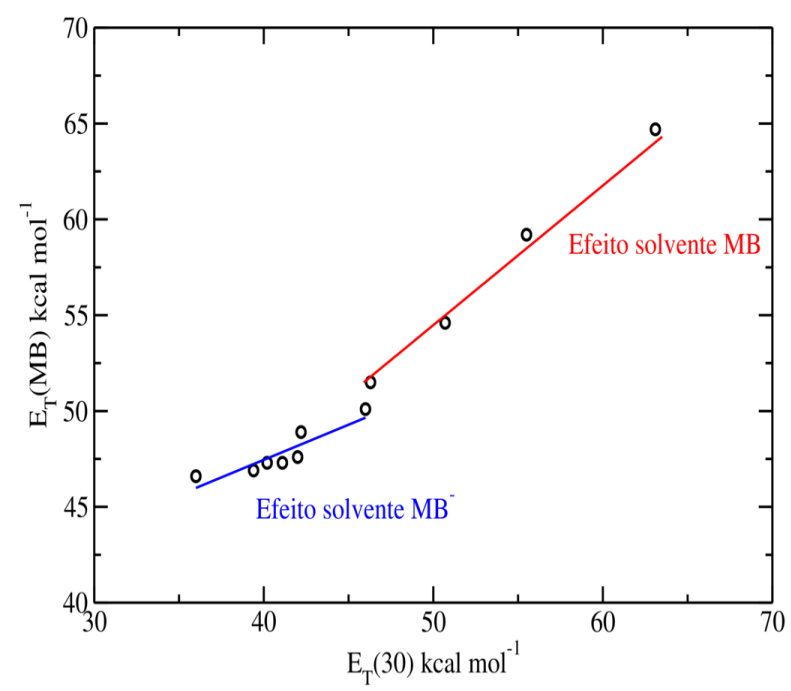

Figura 8.1: Comportamento anômalo para o solvatocromismo da MB.

Com relação aos derivados da MB, nós obtivemos as seguintes conclusões.

- Observamos que o modelo contínuo polarizável (PCM) provoca pequenas deformações estruturais na geometria das sondas estudadas. Por outro lado, as sondas são altamente polarizáveis na presença do meio apresentando grandes mudanças no momento de dipolo;

- Observamos que deformações na geometria provoca um deslocamento para menores energias (ou maiores comprimentos de onda);

- De maneira geral nós conseguimos uma boa descrição teórica para a tendência do deslocamento solvatocrômico observado experimentalmente. A descrição da excitação eletrônica em água e metanol é bem descrita; 
- Vimos no estudo teórico que o dímero das sondas pode ser formado no solvente 1,4 dioxano, baixa polaridade, e a excitação eletrônica obtida para essa estrutura está em boa concordância com os valores experimentais.

- Acreditamos que a formação dos dímeros pode favorecer a transferência de próton $\left(\mathrm{H}^{+}\right)$intradímero, de maneira similar ao que propomos para a MB. 


\section{Referências Bibliográficas}

[1] G. C. Maitland, M. Rigby, E. B. Smith, W. A. Wakeham; Intermolecular ForcesTheir Origin and Determination; Oxford University Press, Oxford, (1987).

[2] H. Ratajczak, W. J. Orville-Thomas; Molecular Interactions; vol. 1-3 Wiley, New York (1980-1982).

[3] M.V.A. Damasceno, B.J.C. Cabral, K.Coutinho; Structure and electronic properties of hydrated mesityl oxide: a sequential quantum mechanics/molecular mechanics approach; Theor. Chem. Acc. 131, 1214 (2012).

[4] S. N. Timasheff; Protein-solvent interactions and protein conformations; Acc. Chem. Res. 3, 62 (1970).

[5] P. Suppan, N. Ghoneim; Solvatochromism; The Royal Society of Chemistry, Londres (1997).

[6] S. E. Sheppard; The Effects of Environment and Aggregation on the Absorption Spectra of Dyes; Rev. Mod. Phys. 14, 303 (1942).

[7] W. L. Archer; Industrial Solvents Handbook; Dekker, New York (1996).

[8] C. Reichardt; Empirical Parameters of the Polarity of Solvent; Angew. Chem. 4, 29 (1965). 
[9] P. L. Novaki, O. A. El Soud; Solvatochromism in Binary Solvent Mixtures: Effects of the Molecular Structure of Probe; Ber. Bunsenges. Phys. Chem. 101 902 (1997).

[10] C. Reichardt; Solvent Effects in Organic Chemistry; Verlag Chemie, Weinheim, New York (1979).

[11] C. Reichardt; Solvatochromic Dyes as Solvent Polarity Indicators; Chem. Rev. 94, 2319 (1994).

[12] E. M. Kosower; An Introduction to Physical Organic Chemistry; Wiley, New York (1968).

[13] A. Streitwieser, C. H. Heathcock, E. M. Kosower; Introduction to Organic Chemistry; McMillan, New York, (1992).

[14] A. D. Buckingham, E. Lippert, E. Bratos, Eds.; Organic Liquids - Structure, Dynamics and Chemical Properties; Wiley, New York (1978).

[15] H. G. Benson, J. N. Murrel; J. Chem. Soc. Faraday Trans. 2 68, 137 (1972).

[16] M. C. Rezende; The Effect of Annulation Upon the Solvatochromic Behaviour of Related Merocyanines; J. Braz. Chem. Soc. 8, 631 (1997).

[17] M. Tsukada, Y. Mineo, K. Itoh; Resonace Raman and surface-enhanced resonance Raman scattering study on the structure of a merocyanine dye, 4-(2-(4hydroxyphenyl)ethenyl)-1-methylpyridinium; J. Phys. Chem. 93, 7989 (1989).

[18] C. Machado, M. D. Nascimento, M. C. Rezende, A. E. Beezer; Calorimetric evidence of aggregation of the ET(30) dye in alcoholic solutions; Thermochim. Acta 328, 155 (1999).

[19] R. Nagarajan, C. C. Wang; Theory of surfactant aggregation in water/ethylene glycol mixed solvents.; Langmuir 16, 5242 (2000). 
[20] Amaresh Mishra, et al; Cyanines during the 1990s: A Review; Chem. Rev. 100, $1973(2000)$.

[21] L. G. S. Brooker et al; Color and Constitution. X. Absorption of the Merocyanines ; J. Am. Chem. Soc. 73, 5332 (1951).

[22] P. Jacques; On the relative contributions of non-specific and specific interactions to the unusual solvatochromism of a typical merocyanine dye; J. Phys. Chem. 90, $5535(1986)$.

[23] N. A. Murugan, J. Kongsted, Z. Rinkevicius, H. Ågren; Demystifying the solvatochromic reversal in Brooker's merocyanine dye; Phys. Chem. Chem. Phys. 13, 1290 (2011).

[24] N. A. Murugan, J. Kongsted, Z. Rinkevicius, K. Aidas, H. Ågren; Modeling the Structure and Absorption Spectra of Stilbazolium Merocyanine in Polar and Nonpolar Solvents Using Hybrid QM/MM Techniques; J. Phys. Chem. B 114, 13349 (2010).

[25] A. Botrel , A. le Beuze, P. Jacques, H. Strub; Solvatochromism of a typical merocyanine dye. A theoretical investigation through the CNDO/SCI method including solvation; J. Chem. Soc., Faraday Trans. 2 80, 1235 (1984).

[26] S. F. Alberti, J. Echave; Theoretical study of the solvatochromism of a merocyanine dye; Chem. Phys. 223, 183 (1997).

[27] T. Wada, H. Nakano, H. Sato; Solvatochromic Shift of Brooker's Merocyanine: Hartree-Fock Exchange in Time Dependent Density Functional Calculation and Hydrogen Bonding Effect; J. Chem. Theory Comput. 10, 4535 (2014). 
[28] K. A. Al-Hassan, M. A. El-Bayoumi; Excited-state Phenomena Associated with Solvation Site Heterogeneity: a large edge-excitation red-shift in a Merocyanine dye-ethanol solution; Chem. Phys. Lett. 76, 121 (1980)

[29] J. O. Morley, R. M. Morley, R. Docherty, M. H. Charlton; Fundamental Studies on Brooker's Merocyanine; J. Am. Chem. Soc. 119, 10192 (1997)

[30] C. T. Martins, M. S. Lima, E. L. Bastos, O. A. El Seoud; ThermoSolvatochromism of Merocyanine Polarity Probes - What are the Consequences of Increasing Probe Lipophilicity through Annelation?; Eur. J. Org. Chem., 1165 (2008).

[31] T. Malaspina, E. E. Fileti, E. L. Bastos; Effect of solute flexibility and polarization on the solvatochromic shift of a brominated merocyanine dye in water: A sequential MD/QM study; Int. J. Quantum Chem. 111, 1607 (2011).

[32] P.L. Silva, P.A.R. Pires, M.A.S. Trassi, O.A. El Seoud; Solvation in pure liquids: What can be learned from the use of pairs of indicators?; J. Phys. Chem. B 112, 14976 (2008).

[33] B. Bussemer, I. Dreiling, U. Grummt, G. Mohr; Spectroscopic and quantum chemical study of the Brønsted acid sites in zeolite L channels with acidochromic cyanine dyes; J. Photochem. Photobio. A: Chem. 204, 90 (2009).

[34] S. Abdel-Halim, M. H. Abdel-Kader, U. E. Steiner; Thermal Cis-Trans Isomerization of Solvatochromic Merocyanlnes: Linear Correlations between Solvent Polarity and Adiabatic and Diabatic Transition Energies; J. Phys. Chem. 92, 4324 (1988).

[35] M. Tsukada, Y. Mineo, K. Itoh; Resonace Raman and surface-enhanced resonance Raman scattering study on the structure of a merocyanine dye, 4-(2-(4hydroxyphenyl)ethenyl)-1-methylpyridinium; J. Phys. Chem. 93, 7989 (1989). 
[36] I. Baraldi, G. Biancolini, F. Momicchioli, G. Ponterini, D. Vanossi; Solvent influence on absorption and fluorescence spectra of merocyanine dyes: a theoretical and experimental study; Chem. Phys. 288, 309 (2003).

[37] H. G. Benson, J. N. Murrell; Some studies of benzenoid-quinonoid resonance. Part 2. - The effect of solvent polarity on the structure and properties of merocyanine dyes; J. Chem. Soc., Faraday Trans. 2 68, 137 (1972).

[38] D. J. Williams; Nonlinear Optical Properties of Organics and Polymeric Materials; American Chemical Society: ACS Symposium Series 233; American Chemical Society: Washington (1983).

[39] J. D. M. Vianna, A. Fazzio, S. Canuto; Teoria Quântica de Moléculas e Sólidos; Livraria da Física, São Paulo (2004).

[40] M. Born, R. Oppenheimer; Quantum theory of molecules; Ann. Phys. Leipzig. 84, 457 (1927).

[41] M. Born, K. Huang; Dynamical Theory of Cristal Lattice; Oxford University Press, N. Y. apêndice VII (1954).

[42] D. R. Hartree; The Wave Mechanics of an Atom with a Non-Coulomb Central Field. Part I-Theory and Methods; Proc. Cambridge Phil. Soc. 24, 89 (1928).

[43] D. R. Hartree; The Wave Mechanics of an Atom with a Non-Coulomb Central Field.Part II-Some Results and Discussion; Proc. Cambridge Phil. Soc. 24, 111 (1928).

[44] A. Szabo, N. S. Ostlund;Modern Quantum Chemistry; Dover, New York (1996).

[45] M. W. Hanna; Quantum Mechanics in Chemistry; 2a Ed., W. A. Benjamin Inc., New York, (1969). 
[46] J. C. Slater; The Theory of Complex Spectra; Phys. Rev. 34, 1293 (1929).

[47] N. H. Morgon, K. Coutinho (Eds); Métodos de Química Teórica e Modelagem Molecular; Livraria da Física, São Paulo (2007).

[48] H. Goldstein; Classical Mechanics, Addison-Wesley, London (1950).

[49] I. N. Levine; Quantum Chemistry; 5a Ed., Prentice Hall, New Jersey (2000).

[50] C. C. J. Roothaan; New Developments in Molecular Orbital Theory, Rev. Mod. Phys. 23, 69 (1951).

[51] C. Froese-Fisher; The Hartree-Fock Methods for Atoms; $1^{\mathrm{a}}$ Ed., John Wiley \& Sons, New York (1977).

[52] P. O. Löwdin; Correlation problem in many-electron quantum mechanics. I. Review of different approaches and discussion of some current ideas; Adv. Chem. Phys. 2, 207 (1959).

[53] S. J. Huzinaga; Gaussian-Type Functions for Polyatomic Systems. I; J. Chem. Phys. 42, 1293 (1965).

[54] W. J. Hehre, L. Randon, P. V. R. Schleyer, J. A. Pople; Ab initio Molecular Orbital Theory; Jonh Wiley \& Sons, New York, (1996).

[55] R. Krishnan, J. S. Binkley, R. Seeger, J. A. Pople; Self-consistent molecular orbital methods. XX. A basis set for correlated wave functions; J. Chem. Phys. $72,650(1980)$.

[56] M. J. Frisch, G. W. Trucks, H. B. Schlegel, G. E. Scuseria, M. A. Robb, J. R. Cheeseman, J. A. Montgomery, Jr., T. Vreven, K. N. Kudin, J. C. Burant, J. M. Millam, S. S. Iyengar, J. Tomasi, V. Barone, B. Mennucci, M. Cossi, G. Scalmani, N. Rega, G. A. Petersson, H. Nakatsuji, M. Hada, M. Ehara, K. 
Toyota, R. Fukuda, J. Hasegawa, M. Ishida, T. Nakajima, Y. Honda, O. Kitao, H. Nakai, M. Klene, X. Li, J. E. Knox, H. P. Hratchian, J. B. Cross, V. Bakken, C. Adamo, J. Jaramillo, R. Gomperts, R. E. Stratmann, O. Yazyev, A. J. Austin, R. Cammi, C. Pomelli, J. W. Ochterski, P. Y. Ayala, K. Morokuma, G. A. Voth, P. Salvador, J. J. Dannenberg, V. G. Zakrzewski, S. Dapprich, A. D. Daniels, M. C. Strain, O. Farkas, D. K. Malick, A. D. Rabuck, K. Raghavachari, J. B. Foresman, J. V. Ortiz, Q. Cui, A. G. Baboul, S. Clifford, J. Cioslowski, B. B. Stefanov, G. Liu, A. Liashenko, P. Piskorz, I. Komaromi, R. L. Martin, D. J. Fox, T. Keith, M. A. Al-Laham, C. Y. Peng, A. Nanayakkara, M. Challacombe, P. M. W. Gill, B. Johnson, W. Chen, M. W. Wong, C. Gonzalez, and J. A. Pople, Gaussian 03, Revision C.02, Gaussian, Inc., Wallingford CT (2004).

[57] C.Møller, M. S. Plesset; Note on an Approximation Treatment for Many-Electron Systems; Phys. Rev. 46, 618 (1934).

[58] R. Krishnan, J. A. Pople; Approximate fourth-order perturbation theory of the electron correlation energy; Int. J. Quantum Chem. 14, 91 (1978).

[59] J. Olsen, O. Christiansen, H. Koch, P. Jorgensen; Surprising cases of divergent behavior in Møller-Plesset perturbation theory; J. Chem. Phys. 105, 5082 (1996).

[60] B. H. Brandow; Linked-Cluster Expansions for the Nuclear Many-Body Problem; Rev. Mod. Phys. 39, 771 (1967).

[61] P. Hohenberg, W. Kohn; Inhomogeneous Electron Gas; Phys. Rev. B 136, B864 (1964).

[62] W. Kohn, L. J. Sham; Self-consistent equations including exchange and correlation effects; Phys. Rev. 140, 1133 (1965)

[63] J. P. Perdew, K. Burke; Comparison shopping for a gradient-corrected density functional; Int. Quantum Chem. 57, 309 (1996). 
[64] J. P. Perdew, K. Burke, M. Ernzenhof; Generalized Gradient Approximation Made Simple; Phys. Rev. Lett. 77, 3865 (1996); 78, 1396 (1997).

[65] C. Lee, W. Yang, R. G. Parr; Development of the Colle-Salvetti correlationenergy formula into a functional of the electron density; Phys. Rev. B 37, 785 (1988)

[66] A. D. Becke; Density-functional thermochemistry. III. The role of exact exchange; J. Chem. Phys. 98, 5648 (1993).

[67] J. P. Perdew, Y. Wang; Accurate and simple density functional for the electronic exchange energy: Generalized gradient approximation; Phys. Rev. B 33, 8800 (1986).

[68] J. P. Perdew; Density-functional approximation for the correlation energy of the inhomogeneous electron gas; Phys. Rev. B 33, 8822 (1986).

[69] A. D. Becke; Density-functional exchange-energy approximation with correct asymptotic behaviour; Phys. Rev. A 38, 3098 (1988).

[70] J. P. Perdew, K. Burke, Y. Wang; Generalized gradient approximation for the exchange-correlation hole of a many-electron system; Phys. Rev. B 54, 16533 (1996).

[71] Y. Imamura, T. Otsuka, H. Nakai; Description of core excitations by timedependent density functional theory with local density approximation, generalized gradient approximation, meta-generalized gradient approximation, and hybrid functionals; J. Comput. Chem. 28, 2067 (2007).

[72] S. H. Vosko, L. Wilk, M. Nusair; Accurate spin-dependent electron liquid correlation energies for local spin density calculations: a critical analysis; Can. J. Phys. 58, 1200 (1980). 
[73] M. Horbatsch, R. M. Dreizler; Time-dependent Thomas-Fermi approach to atomic collisions I. High-energy proton-atom scattering; Z. Phys. A 300, 119 (1992).

[74] G. Holzwarth; Static and dynamical Thomas Fermi theory for nuclei; Phys. Lett. B 66, 29 (1977).

[75] P. Malzacher, R. M. Dreizler; Charge oscillations and photoabsorption of the Thomas-Fermi-Dirac-Weizsäcker atom; Z. Phys. A 307, 211 (1982).

[76] E. Runge, E. K. U. Gross; Density-Functional Theory for Time-Dependent Systems; Phys. Rev. Lett 52, 997 (1984).

[77] M. A. L. Marques, E. K. U. Gross; Time-dependent density functional theory; Annu. Rev. Phys. Chem. 55, 427 (2004).

[78] R. S. Mulliken; Electronic Population Analysis on LCAO[Single Bond]MO Molecular Wave Functions. I; J. Chem. Phys. 23, 1833 (1955).

[79] K. B. Wiberg, P. R. Rablen; A Comparison of Atomic Charges Derived via Different Procedures; J. Comp. Chem.14, 1504 (1993).

[80] D.E. Williams, J. Yan; Adv. Atomic Mol. Phys. 23, 87 (1988).

[81] F. A. Momany; Determination of partial atomic charges from ab initio molecular electrostatic potentials. Application to formamide, methanol, and formic acid; J. Phys. Chem. 82, 592 (1978).

[82] S. R. Cox, D.E. Williams; Representation of the molecular electrostatic potential by a net atomic charge model; J. Comp. Chem. 2, 304 (1981).

[83] C. M. Breneman, K. B. Wiberg; Determining atom-centered monopoles from molecular electrostatic potentials. The need for high sampling density in formamide conformational analysis; J. Comp. Chem. 11, 361 (1990). 
[84] U. C. Singh, P. A. Kollman; An approach to computing electrostatic charges for molecules; J. Comp. Chem. 5, 129 (1984).

[85] M. Born; Volumes and heats of hydration of ions; Z. Phys. 1, 45 (1920).

[86] J. G. Kirkwood; Theory of solutions of molecules containing widely separated charges with special application to amphoteric ions; J. Chem. Phys. 2, 351 (1934).

[87] L. Onsager; Electric Moments of Molecules in Liquids; J. Am. Chem. Soc. 58, $1486(1936)$.

[88] C. Böttcher; Theory of Electronic Polarization; Elsevier, Amsterdan, (1973).

[89] S. Miertus, E. Scrocco, J. Tomasi; Electrostatic Interaction of a Solute with a Continuum. A Direct Utilization of ab initio Molecular Potentials for the Prevision of Solvent Effects; J. Chem. Phys. 55, 117 (1981).

[90] J. Tomasi, R. Cammi, B. Mennuci; Medium Effects on the Properties of Chemical Systems: an Overview of Recent Reformulations in the Polarizable Continuum Model (PCM); Int. J. Quantum Chem. 75, 783 (1999).

[91] S. R. A. Salinas; Introdução à Fúsica Estatística; Editora da Universidade de São Paulo, São Paulo (2005).

[92] J. P. Hansen, I. R. McDonald; Theory of Simple Liquids; Academic Press, London (1986).

[93] T. E. Hill; An Introduction to Statistical Mechanics; Dover, New York (1986).

[94] M. P. Allen, D. J. Tildesley; Computer Simulation of Liquids; Oxford University Press, Oxford, (1987). 
[95] P. Ehrenfest, T. Ehrenfest; The Conceptual Foundations of the Statistical Approach in Mechanics; Dover, New York, (1990).

[96] N. Metropolis; A. W. Rosenbluth; M. N. Rosenbluth; A. H. Teller, E. Teller; Equations of State Calculations by Fast Computing Machines; J. Chem. Phys. 21, 1087 (1953).

[97] H. C. Georg; Efeitos do Meio em Propriedades Conformacionais e Eletrônicas de Moléculas; tese de doutoramento, Universidade de São Paulo, São Paulo (2006).

[98] K. Coutinho, S. Canuto, DICE: Um programa para simulação computacional de líquidos moleculares, utilizando o método Monte Carlo Metropolis e Teoria da Perturbação Termodinâmica para cálculos de energia livre; Universidade de São Paulo (1997).

[99] K. Coutinho; Modelo Discreto de Solvente. Solvatocromismo no Espectro de Absorção Molecular; tese de doutoramento, Universidade de São Paulo, São Paulo (1997).

[100] B. M. Axilrol, E. Teller; Interaction of the van der Waals Type Between Three Atoms; J. Chem. Phys. 11, 299 (1943).

[101] J. E. Lennard-Jones; The equation of state of gases and critical phenomena; Physica 4, 941 (1937).

[102] C. Chatfield; The Analysis of Time Series. An Introduction; Chapman and Hall, London, $3^{\text {a }}$ Ed., (1984).

[103] D. J. A. De Ridder, D. Heijdenrijk, H. Schenk; Structure of 4-2-[1-Methyl4(1H)-pyridylene]ethylidenecyclohexa-2,5-dien-1-one thihydrate; Acta Crystallogr., Sect. C Cryst. Struct. Commum. 46, 2197 (1990). 
[104] W. L. Jorgensen, D. S. Maxwell, J. Tirado-Rives; Development and Testing of the OPLS All-Atom Force Field on Conformational Energetics and Properties of Organic Liquids; J. Am. Chem. Soc. 118, 11225 (1996) e informatic support.

[105] H. J. C. Berendsen, J. P. M. Postma, W. F. van Gunsteren, and J. Hermans; Intermolecular Forces; edited by B. Pullman, Reidel, Dordrecht (1981).

[106] K. Coutinho, H. C. Georg, T. L. Fonseca, V. Ludwig, S. Canuto; An efficient statistically converged average configuration for solvent effects; Chem. Phys. Lett. 437, 148 (2007).

[107] W. L. Jorgensen; Optimized intermolecular potential functions for liquid alcohols; J. Phys. Chem., 90, 1276 (1986).

[108] H.J. Böhm, I.R. McDonald, P. Madden; An effective pair potential for liquid acetonitrile; Mol. Phys., 49, 347 (1983).

[109] V. W. D. Cruzeiro; Estudos Teóricos do Espectro de Absorção de Porfirinas e Ftalocianinas; Dissertação de mestrado, Universidade de São Paulo (2014).

[110] J. Ho, M. L. Coote; A universal approach for continuum solvent $p$ Ka calculations: are we there yet?; Theor. Chem. Acc. 125, 3 (2010).

[111] J. R. Pliego Jr.; Thermodynamic cycles and the calculation of $p K_{a}$; Chem. Phys. Lett. 367, 145 (2003).

[112] A. Ben-Naim; Standard thermodynamics of transfer: Uses and misuses; J. Phys. Chem. 82, 792 (1978).

[113] A. Ben-Naim, Y. Marcus; Solvation thermodynamics of non-ionic solutes; Chem. Phys. 81, 2016 (1984). 
[114] D. M. Camaioni, C. A. Schwerdtfeger; Comment on accurate experimental values for the free energies of $\mathrm{H}^{+}, \mathrm{OH}^{-}$and $\mathrm{H}_{3} \mathrm{O}^{+} ; \mathrm{J}$. Phys. Chem. A 109, 10795 (2005).

[115] J. R. Pliego Jr., J. M. Riveros; On the Calculation of the Absolute Solvation Free Energy of Ionic Species: Application of the Extrapolation Method to the Hydroxide Ion in Aqueous Solution; J. Phys. Chem. B. 104, 5155 (2000).

[116] V. Barone, M. Cossi, J. Tomasi; A New Definition of Cavities for the Computation of Solvation Free Energies by the Polarizable Continuum Model; J. Chem. Phys. 107, 3210 (1997).

[117] M. D. Tissander, K. A. Cowen, W. Y. Feng, E. Gundlach, M. H. Cohen, A. D. Earhart, J. V. Coe, T. R. Tutle; The proton's absolute aqueous enthalpy and Gibbs free energy of solvation from cluster-ion solvation data; J. Phys. Chem. A 102, 7787 (1998).

[118] C. P. Kelly, C. J. Cramer, D. G. Truhlar; Aqueous solvation free energies of ions and ion-water clusters based on an accurate value for the absolute aqueous solvation free energy of the proton, J. Phys. Chem. B 110, 16066 (2006).

[119] L. B. A. Oliveira, T. L. Fonseca, K. Coutinho, S. Canuto; A sequential MC/TDDFT study of the solvatochromic shift of the pyridinium- $N$-phenoxide betaine dye in water using standard and long-range corrected functionals; Chem. Phys. Lett. $\mathbf{5 1 4}, 251$ (2011).

[120] V. Manzoni, M. L. Lyra, R. M. Gester, K. Coutinho, S. Canuto; Study of the optical and magnetic properties of pyrimidine in water combining PCM and $Q M / M M$ methodologies; Phys. Chem. Chem. Phys. 12, 14023 (2010).

[121] R. C. Barreto, K. Coutinho, H. C. Georg, S. Canuto; Combined Monte Carlo and quantum mechanics study of the solvatochromism of phenol in water. The 
origin of the blue shift of the lowest pi-pi* transition; Phys. Chem. Chem. Phys. 11, 1338 (2009).

[122] V. Manzoni, M. L. Lyra, K. Coutinho, S. Canuto; Comparison of polarizable continuum model and quantum mechanics/molecular mechanics solute electronic polarization: Study of the optical and magnetic properties of diazines in water;J. Chem. Phys. 135, 144103 (2011).

[123] M. Luhmer, J. Reisse; Molecular Dynamics Simulation Study of the NMR Relaxation of Xenon-131 Dissolved in 1,3-Dioxane and 1,4-Dioxane; Journal of Magnetic Resonance, Series A, 105, 197 (2005).

[124] W. L. Jorgensen, D. S. Maxwell, J. Tirado-Rives; A comprehensive study of the rotational energy profiles of organic systems by ab initio MO theory, forming a basis for peptide torsional parameters; J. Comput. Chem. 16, 984(1995).

[125] M. Mezei, D. L. Beveridge; Theoretical studies of hydrogen bonding in liquid water and aqueous solution; J. Chem. Phys. 74, 622 (1981).

[126] M. G. Sceats, S. A. Rice; The water-water pair potential near the hydrogen bonded equilibrium configuration; J. Chem. Phys. 72, 3236 (1981).

[127] K. Coutinho, S. Canuto; The sequential Monte Carlo-quantum Mechanics Methodology. Application to the Solvent Effects in the Stokes Shift of Acetone in Water; J. Mol. Struct. (Theochem) 632, 235 (2003).

[128] H. C. Georg, K. Coutinho, S. Canuto; A sequential Monte Carlo quantum mechanics study of the hydrogen-bond interaction and the solvatochromic shift of the $n-\pi^{*}$ transition of acrolein in water; J. Chem. Phys. 123, 124307 (2005).

[129] E. Buncel, S. Rajagopal; Solvatochromism and solvent polarity scales; Acc. Chem. Res. 23, 226 (1990). 
[130] D. C. Harris; Análise Química Quantitativa. LTC Ed., Rio de Janeiro, 5 ${ }^{\mathrm{a}}$ Ed., (2001).

[131] W. Masterton, C. Hurley, E. Neth; Chemistry: Principles and Reactions Cengage Learning, $7^{\mathrm{a}}$ Ed., (2011).

[132] P. R. R. Costa, V. F. Ferreira, P. M. Esteves, M. L. A. A. Vasconcellos; Ácidos e Bases em Química Orgânica. Bookman, Porto Alegre, (2005).

[133] L. J. Henderson; Concerning the relationship between the strength of acids and their capacity to preserve neutrality; Am. J. Physiol. 21, 173 (1908)

[134] N. Henry Po, N. M. Senozan; Henderson-Hasselbalch Equation: Its History and Limitations; J. Chem. Educ. 78, 1499 (2001).

[135] J. L. Crammer, A. Neuberger; Biochem. J. 37, 302 (1943).

[136] A. R. da Cunha, E. L. Duarte, M. Teresa Lamy, K. Coutinho; Protonation/deprotonation process of Emodin in aqueous solution and $p$ Ka determination: UV/Visible spectrophotometric titration and quantum/molecular mechanics calculations; Chem. Phys. 440, 69 (2014).

[137] P. W. Atkins; General Chemistry; Scientific American, New York, (1990).

[138] H. H. Willard, L. L. Merrit Jr., J. A. Dean, F. A. Settle Jr.; Instrumental Methods of Analysis; Wadsworth Pu. Co., California, $7^{\mathrm{a}}$ Ed., (1988) 


\section{Apêndice A}

\section{Parâmetros Usados na Simulação.}

Nas tabelas a seguir apresentamos os dados usados para a simulação da sonda MB e derivados utilizando o programa DICE. Cada átomo é caracterizado pelo seu número atômico, pelas coordenadas xyz $(\AA)$, pela sua carga, e pelos parâmetros $\epsilon(\mathrm{kcal} / \mathrm{mol})$ e $\sigma(\AA)$ do potencial de interação intermolecular. Aqui, a geometria e o conjunto de cargas foram obtidos na presença do respectivo solvente e os parâmetros $\epsilon(\mathrm{kcal} / \mathrm{mol})$ e $\sigma(\AA)$ foram obtidos do campo de força OPLS [104. Apresentamos os arquivos para simulações em diferentes solventes considerando a MB nas formas neutra e protonada.

\section{A.1 Molécula neutra: MB.}


Tabela A.1: Tabela com os parâmetros necessários para realizar uma simulação no DICE. Sonda MB, geometria e cargas obtidas na presença do solvente através do modelo contínuo PCM, solvente água.

\begin{tabular}{|c|c|c|c|c|c|c|}
\hline $\mathrm{N}^{\mathrm{o}}$ atômico & & Coordenadas & $\operatorname{xyz}(\AA)$ & Cargas(e) & $\epsilon(\mathrm{kcal} / \mathrm{mol})$ & $\sigma(\AA)$ \\
\hline 6 & 4.060905 & 1.155150 & -0.005114 & -0.011278 & 0.070 & 3.550 \\
\hline 6 & 2.711820 & 1.371332 & 0.000194 & -0.213234 & 0.070 & 3.550 \\
\hline 6 & 1.783474 & 0.289091 & -0.003360 & 0.299472 & 0.076 & 3.550 \\
\hline 6 & 2.373079 & -1.010101 & -0.013594 & -0.200067 & 0.070 & 3.550 \\
\hline 6 & 3.729666 & -1.169264 & -0.019386 & -0.032408 & 0.070 & 3.550 \\
\hline 7 & 4.579439 & -0.104183 & -0.019269 & 0.128020 & 0.170 & 3.300 \\
\hline 6 & 6.036311 & -0.310095 & 0.033844 & -0.169354 & 0.066 & 3.500 \\
\hline 6 & 0.386683 & 0.551296 & -0.000693 & -0.334134 & 0.076 & 3.550 \\
\hline 6 & -0.606627 & -0.405637 & 0.000211 & -0.114663 & 0.076 & 3.550 \\
\hline 6 & -2.011841 & -0.211155 & 0.001180 & 0.093688 & 0.076 & 3.550 \\
\hline 6 & -2.875219 & -1.346454 & 0.005843 & -0.210652 & 0.070 & 3.550 \\
\hline 6 & -2.640487 & 1.070695 & -0.002716 & -0.155696 & 0.070 & 3.550 \\
\hline 6 & -4.241402 & -1.229639 & 0.006659 & -0.291033 & 0.070 & 3.550 \\
\hline 6 & -4.002687 & 1.205846 & -0.002376 & -0.304099 & 0.070 & 3.550 \\
\hline 6 & -4.893803 & 0.061020 & 0.002102 & 0.655428 & 0.105 & 3.750 \\
\hline 8 & -6.152438 & 0.189085 & 0.003383 & -0.839008 & 0.210 & 2.960 \\
\hline 1 & 4.777223 & 1.964975 & -0.001073 & 0.150398 & 0.030 & 2.420 \\
\hline 1 & 2.354027 & 2.392945 & 0.004779 & 0.152377 & 0.030 & 2.420 \\
\hline
\end{tabular}


Tabela A.1 - Continuação da página anterior

\begin{tabular}{|c|c|c|c|c|c|c|}
\hline $\mathrm{N}^{\mathrm{o}}$ atômico & & Coordenadas & $\mathrm{xyz}(\AA)$ & Cargas(e) & $\epsilon(\mathrm{kcal} / \mathrm{mol})$ & $\sigma(\AA)$ \\
\hline 1 & 1.765540 & -1.903934 & -0.021113 & 0.160287 & 0.030 & 2.420 \\
\hline 1 & 4.194009 & -2.145457 & -0.027753 & 0.152305 & 0.030 & 2.420 \\
\hline 1 & 6.375271 & -0.317687 & 1.071027 & 0.114290 & 0.030 & 2.420 \\
\hline 1 & 6.530891 & 0.493994 & -0.507334 & 0.113247 & 0.030 & 2.420 \\
\hline 1 & 6.279153 & -1.260337 & -0.436613 & 0.117691 & 0.030 & 2.500 \\
\hline 1 & 0.109993 & 1.600405 & 0.001234 & 0.155197 & 0.030 & 2.500 \\
\hline 1 & -0.300346 & -1.449222 & 0.000800 & 0.115091 & 0.030 & 2.500 \\
\hline 1 & -2.424179 & -2.335239 & 0.009107 & 0.124456 & 0.030 & 2.420 \\
\hline 1 & -2.026557 & 1.965257 & -0.006699 & 0.117712 & 0.030 & 2.420 \\
\hline 1 & -4.872525 & -2.112630 & 0.010583 & 0.110349 & 0.030 & 2.420 \\
\hline 1 & -4.458291 & 2.191020 & -0.005895 & 0.115619 & 0.030 & 2.420 \\
\hline
\end{tabular}


Tabela A.2: Tabela com os parâmetros necessários para realizar uma simulação no DICE. Sonda MB, geometria e cargas obtidas na presença do solvente através do modelo contínuo PCM, solvente metanol.

\begin{tabular}{|c|c|c|c|c|c|c|}
\hline $\mathrm{N}^{\circ}$ atômico & & Coordenadas & xyz $(\AA)$ & Cargas(e) & $\epsilon(\mathrm{kcal} / \mathrm{mol})$ & $\sigma(\AA)$ \\
\hline 6 & 4.060403 & 1.155282 & -0.005098 & -0.013455 & 0.070 & 3.550 \\
\hline 6 & 2.711798 & 1.371782 & -0.000362 & -0.212433 & 0.070 & 3.550 \\
\hline 6 & 1.782173 & 0.289664 & -0.004290 & 0.289976 & 0.076 & 3.550 \\
\hline 6 & 2.372423 & -1.010072 & -0.014713 & -0.191105 & 0.070 & 3.550 \\
\hline 6 & 3.728609 & -1.169448 & -0.019942 & -0.041731 & 0.070 & 3.550 \\
\hline 7 & 4.579306 & -0.104511 & -0.019253 & 0.129773 & 0.170 & 3.300 \\
\hline 6 & 6.035700 & -0.310556 & 0.036246 & -0.170914 & 0.066 & 3.500 \\
\hline 6 & 0.386780 & 0.551730 & -0.001698 & -0.334445 & 0.076 & 3.550 \\
\hline 6 & -0.607523 & -0.405817 & -0.000726 & -0.109395 & 0.076 & 3.550 \\
\hline 6 & -2.011343 & -0.211472 & 0.000598 & 0.100513 & 0.076 & 3.550 \\
\hline 6 & -2.875320 & -1.347151 & 0.005629 & -0.214438 & 0.070 & 3.550 \\
\hline 6 & -2.640037 & 1.071057 & -0.003085 & -0.161676 & 0.070 & 3.550 \\
\hline 6 & -4.240819 & -1.230274 & 0.007054 & -0.283421 & 0.070 & 3.550 \\
\hline 6 & -4.001587 & 1.206466 & -0.002174 & -0.296113 & 0.070 & 3.550 \\
\hline 6 & -4.893289 & 0.061193 & 0.002780 & 0.651283 & 0.105 & 3.750 \\
\hline 8 & -6.150621 & 0.189312 & 0.004778 & -0.827692 & 0.210 & 2.960 \\
\hline 1 & 4.776896 & 1.965005 & -0.000606 & 0.148827 & 0.030 & 2.420 \\
\hline 1 & 2.354275 & 2.393523 & 0.004170 & 0.151229 & 0.030 & 2.420 \\
\hline
\end{tabular}


Tabela A.2 - Continuação da página anterior

\begin{tabular}{|c|c|c|c|c|c|c|}
\hline $\mathrm{N}^{\mathrm{o}}$ atômico & & Coordenadas & xyz $(\AA)$ & Cargas(e) & $\epsilon(\mathrm{kcal} / \mathrm{mol})$ & $\sigma(\AA)$ \\
\hline 1 & 1.764676 & -1.903779 & -0.022786 & 0.157813 & 0.030 & 2.420 \\
\hline 1 & 4.192600 & -2.145855 & -0.028345 & 0.152308 & 0.030 & 2.420 \\
\hline 1 & 6.373723 & -0.319014 & 1.073836 & 0.113665 & 0.030 & 2.420 \\
\hline 1 & 6.531466 & 0.493769 & -0.503629 & 0.112585 & 0.030 & 2.420 \\
\hline 1 & 6.279655 & -1.260368 & -0.434669 & 0.117594 & 0.030 & 2.500 \\
\hline 1 & 0.109685 & 1.600720 & 0.000380 & 0.154390 & 0.030 & 2.500 \\
\hline 1 & -0.300926 & -1.449329 & -0.000210 & 0.110225 & 0.030 & 2.500 \\
\hline 1 & -2.424107 & -2.335872 & 0.008724 & 0.124584 & 0.030 & 2.420 \\
\hline 1 & -2.025660 & 1.965297 & -0.007366 & 0.118314 & 0.030 & 2.420 \\
\hline 1 & -4.872637 & -2.112658 & 0.011291 & 0.109191 & 0.030 & 2.420 \\
\hline 1 & -4.457632 & 2.191338 & -0.005560 & 0.114547 & 0.030 & 2.420 \\
\hline
\end{tabular}


Tabela A.3: Tabela com os parâmetros necessários para realizar uma simulação no DICE. Sonda MB, geometria e cargas obtidas na presença do solvente através do modelo contínuo PCM, solvente acetonitrila.

\begin{tabular}{|c|c|c|c|c|c|c|}
\hline No atômico & & Coordenadas & xyz $(\AA)$ & Cargas(e) & $\epsilon(\mathrm{kcal} / \mathrm{mol})$ & $\sigma(\AA)$ \\
\hline 6 & 4.060491 & 1.155113 & -0.006069 & -0.012398 & 0.070 & 3.550 \\
\hline 6 & 2.711815 & 1.371510 & -0.001110 & -0.213793 & 0.070 & 3.550 \\
\hline 6 & 1.782340 & 0.289348 & -0.003926 & 0.300398 & 0.076 & 3.550 \\
\hline 6 & 2.372589 & -1.010261 & -0.013265 & -0.198823 & 0.070 & 3.550 \\
\hline 6 & 3.728861 & -1.169546 & -0.018694 & -0.034144 & 0.070 & 3.550 \\
\hline 7 & 4.579354 & -0.104597 & -0.019165 & 0.124914 & 0.170 & 3.300 \\
\hline 6 & 6.035919 & -0.309924 & 0.035290 & -0.169320 & 0.066 & 3.500 \\
\hline 6 & 0.386836 & 0.551585 & -0.001313 & -0.341211 & 0.076 & 3.550 \\
\hline 6 & -0.607524 & -0.405771 & -0.000532 & -0.110805 & 0.076 & 3.550 \\
\hline 6 & -2.011460 & -0.211377 & 0.000801 & 0.093412 & 0.076 & 3.550 \\
\hline 6 & -2.875391 & -1.347038 & 0.004715 & -0.210256 & 0.070 & 3.550 \\
\hline 6 & -2.640160 & 1.071086 & -0.002080 & -0.151457 & 0.070 & 3.550 \\
\hline 6 & -4.240961 & -1.230134 & 0.006044 & -0.286659 & 0.070 & 3.550 \\
\hline 6 & -4.001776 & 1.206393 & -0.001213 & -0.304257 & 0.070 & 3.550 \\
\hline 6 & -4.893347 & 0.061199 & 0.003222 & 0.654153 & 0.105 & 3.750 \\
\hline 8 & -6.150954 & 0.189360 & 0.003851 & -0.829120 & 0.210 & 2.960 \\
\hline 1 & 4.776904 & 1.964927 & -0.002686 & 0.149307 & 0.030 & 2.420 \\
\hline 1 & 2.354175 & 2.393221 & 0.002645 & 0.150821 & 0.030 & 2.420 \\
\hline
\end{tabular}


Tabela A.3 - Continuação da página anterior

\begin{tabular}{|c|c|c|c|c|c|c|}
\hline $\mathrm{N}^{\circ}$ atômico & & Coordenadas & xyz $(\AA)$ & Cargas(e) & $\epsilon(\mathrm{kcal} / \mathrm{mol})$ & $\sigma(\AA)$ \\
\hline 1 & 1.764963 & -1.904074 & -0.020168 & 0.158286 & 0.030 & 2.420 \\
\hline 1 & 4.192871 & -2.145937 & -0.026384 & 0.150973 & 0.030 & 2.420 \\
\hline 1 & 6.376329 & -0.305688 & 1.072091 & 0.113579 & 0.030 & 2.420 \\
\hline 1 & 6.530527 & 0.487601 & -0.515675 & 0.112411 & 0.030 & 2.420 \\
\hline 1 & 6.278593 & -1.265510 & -0.424347 & 0.117627 & 0.030 & 2.500 \\
\hline 1 & 0.109766 & 1.600602 & 0.000654 & 0.156734 & 0.030 & 2.500 \\
\hline 1 & -0.300936 & -1.449302 & -0.000448 & 0.113887 & 0.030 & 2.500 \\
\hline 1 & -2.424023 & -2.335694 & 0.006932 & 0.123664 & 0.030 & 2.420 \\
\hline 1 & -2.025731 & 1.965315 & -0.005649 & 0.115935 & 0.030 & 2.420 \\
\hline 1 & -4.872801 & -2.112444 & 0.009227 & 0.109639 & 0.030 & 2.420 \\
\hline 1 & -4.457884 & 2.191184 & -0.004060 & 0.116501 & 0.030 & 2.420 \\
\hline
\end{tabular}




\section{A.2 Molécula protonada: $\mathrm{MBH}^{+}$.}

Tabela A.4: Tabela com os parâmetros necessários para realizar uma simulação no DICE. Sonda $\mathrm{MBH}^{+}$, geometria e cargas obtidas na presença do solvente através do modelo contínuo PCM, solvente água.

\begin{tabular}{|c|c|c|c|c|c|c|}
\hline $\mathrm{N}^{\mathrm{o}}$ atômico & & Coordenadas & xyz $(\AA)$ & Cargas(e) & $\epsilon(\mathrm{kcal} / \mathrm{mol})$ & $\sigma(\AA)$ \\
\hline 6 & 4.113785 & 1.157191 & 0.022892 & 0.031667 & 0.070 & 3.550 \\
\hline 6 & 2.755602 & 1.361347 & 0.023373 & -0.221559 & 0.070 & 3.550 \\
\hline 6 & 1.856664 & 0.272445 & -0.006890 & 0.324530 & 0.076 & 3.550 \\
\hline 6 & 2.439433 & -1.015210 & -0.041279 & -0.193795 & 0.070 & 3.550 \\
\hline 6 & 3.802482 & -1.165127 & -0.042172 & -0.002351 & 0.070 & 3.550 \\
\hline 7 & 4.632842 & -0.092580 & -0.011981 & 0.135950 & 0.170 & 3.300 \\
\hline 6 & 6.096001 & -0.293258 & 0.033534 & -0.182923 & 0.066 & 3.500 \\
\hline 6 & 0.432852 & 0.528105 & -0.005817 & -0.255655 & 0.076 & 3.550 \\
\hline 6 & -0.534023 & -0.421406 & -0.002099 & -0.143141 & 0.076 & 3.550 \\
\hline 6 & -1.970496 & -0.212805 & -0.001114 & 0.194287 & 0.076 & 3.550 \\
\hline 6 & -2.816659 & -1.337864 & 0.027610 & -0.210955 & 0.070 & 3.550 \\
\hline 6 & -2.575883 & 1.062171 & -0.028910 & -0.210371 & 0.070 & 3.550 \\
\hline 6 & -4.198527 & -1.209668 & 0.031103 & -0.242231 & 0.070 & 3.550 \\
\hline 6 & -3.951088 & 1.202492 & -0.026617 & -0.187082 & 0.070 & 3.550 \\
\hline 6 & -4.771226 & 0.065272 & 0.004343 & 0.430238 & 0.105 & 3.750 \\
\hline 8 & -6.115140 & 0.269018 & 0.005872 & -0.670713 & 0.210 & 2.960 \\
\hline 1 & 4.823216 & 1.972085 & 0.048109 & 0.156503 & 0.030 & 2.420 \\
\hline
\end{tabular}




$\begin{array}{lllllll}1 & 2.386691 & 2.378180 & 0.046560 & 0.176555 & 0.030 & 2.420\end{array}$


Tabela A.4 - Continuação da página anterior

\begin{tabular}{|c|c|c|c|c|c|c|}
\hline No atômico & & Coordenadas & xyz $(\AA)$ & Cargas(e) & $\epsilon(\mathrm{kcal} / \mathrm{mol})$ & $\sigma(\AA)$ \\
\hline 1 & 1.836504 & -1.911291 & -0.073329 & 0.178796 & 0.030 & 2.420 \\
\hline 1 & 4.276752 & -2.135784 & -0.069458 & 0.162520 & 0.030 & 2.420 \\
\hline 1 & 6.412711 & -0.415246 & 1.069533 & 0.128756 & 0.030 & 2.420 \\
\hline 1 & 6.585223 & 0.573135 & -0.404299 & 0.127170 & 0.030 & 2.420 \\
\hline 1 & 6.349464 & -1.182269 & -0.538864 & 0.128896 & 0.030 & 2.500 \\
\hline 1 & 0.157226 & 1.577059 & -0.002724 & 0.157910 & 0.030 & 2.500 \\
\hline 1 & -0.237584 & -1.466504 & 0.004549 & 0.121503 & 0.030 & 2.500 \\
\hline 1 & -2.379949 & -2.330279 & 0.048313 & 0.149512 & 0.030 & 2.420 \\
\hline 1 & -1.965612 & 1.956497 & -0.054538 & 0.139994 & 0.030 & 2.420 \\
\hline 1 & -6.585187 & -0.574053 & 0.030216 & 0.462108 & 0.000 & 0.000 \\
\hline 1 & -4.410813 & 2.183115 & -0.049068 & 0.159919 & 0.030 & 2.420 \\
\hline 1 & -4.830911 & -2.090824 & 0.054147 & 0.153962 & 0.030 & 2.420 \\
\hline
\end{tabular}


Tabela A.5: Tabela com os parâmetros necessários para realizar uma simulação no DICE. Sonda $\mathrm{MBH}^{+}$, geometria e cargas obtidas na presença do solvente através do modelo contínuo PCM, solvente metanol.

\begin{tabular}{|c|c|c|c|c|c|c|}
\hline $\mathrm{N}^{\circ}$ atômico & & Coordenadas & $\operatorname{xyz}(\AA)$ & Cargas(e) & $\epsilon(\mathrm{kcal} / \mathrm{mol})$ & $\sigma(\AA)$ \\
\hline 6 & 4.110503 & 1.156963 & -0.011501 & 0.027025 & 0.070 & 3.550 \\
\hline 6 & 2.757100 & 1.364669 & -0.007567 & -0.190287 & 0.070 & 3.550 \\
\hline 6 & 1.843185 & 0.278104 & -0.004808 & 0.291119 & 0.076 & 3.550 \\
\hline 6 & 2.433497 & -1.014445 & -0.007259 & -0.176955 & 0.070 & 3.550 \\
\hline 6 & 3.792883 & -1.167465 & -0.011633 & 0.003591 & 0.070 & 3.550 \\
\hline 7 & 4.634037 & -0.097355 & -0.016446 & 0.125883 & 0.170 & 3.300 \\
\hline 6 & 6.095589 & -0.298658 & 0.035384 & -0.175492 & 0.066 & 3.500 \\
\hline 6 & 0.434238 & 0.536545 & -0.002938 & -0.250682 & 0.076 & 3.550 \\
\hline 6 & -0.544937 & -0.414040 & -0.002587 & -0.125673 & 0.076 & 3.550 \\
\hline 6 & -1.968227 & -0.211062 & -0.000328 & 0.161807 & 0.076 & 3.550 \\
\hline 6 & -2.813484 & -1.341832 & -0.001667 & -0.198392 & 0.070 & 3.550 \\
\hline 6 & -2.578729 & 1.066701 & 0.003190 & -0.185911 & 0.070 & 3.550 \\
\hline 6 & -4.190741 & -1.217022 & 0.000202 & -0.240716 & 0.070 & 3.550 \\
\hline 6 & -3.948492 & 1.203639 & 0.005070 & -0.190246 & 0.070 & 3.550 \\
\hline 6 & -4.767556 & 0.060123 & 0.003496 & 0.432852 & 0.105 & 3.750 \\
\hline 8 & -6.097666 & 0.267512 & 0.005457 & -0.666664 & 0.210 & 2.960 \\
\hline 1 & 4.821287 & 1.972304 & -0.012945 & 0.152673 & 0.030 & 2.420 \\
\hline 1 & 2.392045 & 2.383728 & -0.009289 & 0.163339 & 0.030 & 2.420 \\
\hline
\end{tabular}


Tabela A.5 - Continuação da página anterior

\begin{tabular}{|c|c|c|c|c|c|c|}
\hline No atômico & & Coordenadas & xyz $(\AA)$ & Cargas(e) & $\epsilon(\mathrm{kcal} / \mathrm{mol})$ & $\sigma(\AA)$ \\
\hline 1 & 1.828446 & -1.910189 & -0.008743 & 0.170404 & 0.030 & 2.420 \\
\hline 1 & 4.262945 & -2.141670 & -0.013431 & 0.156094 & 0.030 & 2.420 \\
\hline 1 & 6.425356 & -0.372347 & 1.073157 & 0.126357 & 0.030 & 2.420 \\
\hline 1 & 6.589848 & 0.542128 & -0.447324 & 0.125180 & 0.030 & 2.420 \\
\hline 1 & 6.351157 & -1.213065 & -0.496801 & 0.126797 & 0.030 & 2.500 \\
\hline 1 & 0.156413 & 1.584786 & -0.002016 & 0.160995 & 0.030 & 2.500 \\
\hline 1 & -0.245475 & -1.459131 & -0.004568 & 0.118324 & 0.030 & 2.500 \\
\hline 1 & -2.374296 & -2.333555 & -0.004273 & 0.147382 & 0.030 & 2.420 \\
\hline 1 & -1.969898 & 1.962348 & 0.004490 & 0.131657 & 0.030 & 2.420 \\
\hline 1 & -6.583716 & -0.566091 & 0.004088 & 0.465274 & 0.000 & 0.000 \\
\hline 1 & -4.418900 & 2.178827 & 0.007719 & 0.160836 & 0.030 & 2.420 \\
\hline 1 & -4.821122 & -2.100014 & -0.000923 & 0.153425 & 0.030 & 2.420 \\
\hline
\end{tabular}


Tabela A.6: Tabela com os parâmetros necessários para realizar uma simulação no DICE. Sonda $\mathrm{MBH}^{+}$, geometria e cargas obtidas na presença do solvente através do modelo contínuo PCM, solvente acetonitrila.

\begin{tabular}{|c|c|c|c|c|c|c|}
\hline No atômico & & Coordenadas & xyz $(\AA)$ & Cargas(e) & $\epsilon(\mathrm{kcal} / \mathrm{mol})$ & $\sigma(\AA)$ \\
\hline 6 & 4.113808 & 1.156904 & 0.018266 & 0.024284 & 0.070 & 3.550 \\
\hline 6 & 2.755845 & 1.361323 & 0.018929 & -0.191702 & 0.070 & 3.550 \\
\hline 6 & 1.856346 & 0.272431 & -0.006742 & 0.297955 & 0.076 & 3.550 \\
\hline 6 & 2.439091 & -1.015448 & -0.036234 & -0.183172 & 0.070 & 3.550 \\
\hline 6 & 3.802101 & -1.165640 & -0.037460 & 0.008518 & 0.070 & 3.550 \\
\hline 7 & 4.632810 & -0.093238 & -0.012076 & 0.123556 & 0.170 & 3.300 \\
\hline 6 & 6.096109 & -0.292618 & 0.033864 & -0.158469 & 0.066 & 3.500 \\
\hline 6 & 0.432937 & 0.528389 & -0.005881 & -0.245268 & 0.076 & 3.550 \\
\hline 6 & -0.534308 & -0.421029 & -0.003121 & -0.133542 & 0.076 & 3.550 \\
\hline 6 & -1.970467 & -0.212587 & -0.001713 & 0.166793 & 0.076 & 3.550 \\
\hline 6 & -2.816532 & -1.337892 & 0.022999 & -0.188480 & 0.070 & 3.550 \\
\hline 6 & -2.576030 & 1.062504 & -0.024839 & -0.198681 & 0.070 & 3.550 \\
\hline 6 & -4.198279 & -1.209805 & 0.026829 & -0.243268 & 0.070 & 3.550 \\
\hline 6 & -3.951099 & 1.202695 & -0.022126 & -0.177412 & 0.070 & 3.550 \\
\hline 6 & -4.771151 & 0.065205 & 0.004584 & 0.419499 & 0.105 & 3.750 \\
\hline 8 & -6.114758 & 0.268796 & 0.006741 & -0.666084 & 0.210 & 2.960 \\
\hline 1 & 4.823528 & 1.971731 & 0.039713 & 0.155671 & 0.030 & 2.420 \\
\hline 1 & 2.387142 & 2.378331 & 0.038367 & 0.165408 & 0.030 & 2.420 \\
\hline
\end{tabular}


Tabela A.6 - Continuação da página anterior

\begin{tabular}{|c|c|c|c|c|c|c|}
\hline No atômico & & Coordenadas & xyz $(\AA)$ & Cargas(e) & $\epsilon(\mathrm{kcal} / \mathrm{mol})$ & $\sigma(\AA)$ \\
\hline 1 & 1.836023 & -1.911594 & -0.063753 & 0.173015 & 0.030 & 2.420 \\
\hline 1 & 4.276051 & -2.136540 & -0.061320 & 0.156407 & 0.030 & 2.420 \\
\hline 1 & 6.416911 & -0.385557 & 1.071632 & 0.122262 & 0.030 & 2.420 \\
\hline 1 & 6.583871 & 0.560970 & -0.430182 & 0.121675 & 0.030 & 2.420 \\
\hline 1 & 6.347489 & -1.197387 & -0.514146 & 0.122831 & 0.030 & 2.500 \\
\hline 1 & 0.157399 & 1.577350 & -0.002789 & 0.158764 & 0.030 & 2.500 \\
\hline 1 & -0.237869 & -1.466154 & 0.001815 & 0.121515 & 0.030 & 2.500 \\
\hline 1 & -2.379776 & -2.330361 & 0.040248 & 0.142294 & 0.030 & 2.420 \\
\hline 1 & -1.965841 & 1.956986 & -0.046838 & 0.133429 & 0.030 & 2.420 \\
\hline 1 & -6.584922 & -0.574279 & 0.027878 & 0.461466 & 0.000 & 0.000 \\
\hline 1 & -4.411128 & 2.183229 & -0.040875 & 0.157757 & 0.030 & 2.420 \\
\hline 1 & -4.830695 & -2.091014 & 0.046726 & 0.152980 & 0.030 & 2.420 \\
\hline
\end{tabular}


Tabela A.7: Parâmetros obtidos do modelo SPC para a água [105].

\begin{tabular}{|c|c|c|c|c|c|c|}
\hline $\mathrm{N}^{\circ}$ atômico & & Coordenar & $\operatorname{as} x y z(\AA)$ & Cargas(e) & $\epsilon(\mathrm{kcal} / \mathrm{mol})$ & $\sigma(\AA)$ \\
\hline 8 & 0.0000 & 0.0000 & 0.0000 & -0.820 & 0.1550 & 3.1650 \\
\hline 1 & 0.5774 & 0.8165 & 0.0000 & 0.410 & 0.0000 & 0.0000 \\
\hline 1 & 0.5774 & -0.8165 & 0.0000 & 0.410 & 0.0000 & 0.0000 \\
\hline
\end{tabular}

Tabela A.8: Parâmetros obtidos para o solvente metanol [107].

\begin{tabular}{|c|c|c|c|c|c|c|}
\hline $\mathrm{N}^{0}$ atômico & \multicolumn{3}{|c|}{ Coordenadas xyz $(\AA)$} & Cargas(e) & $\epsilon(\mathrm{kcal} / \mathrm{mol})$ & $\sigma(\AA)$ \\
\hline 8 & 1.837572 & -0.054784 & -0.590399 & -0.700 & 0.170 & 3.070 \\
\hline 1 & 1.233853 & 0.719007 & -0.539438 & 0.435 & 0.000 & 0.000 \\
\hline 6 & 2.743241 & 0.010645 & 0.509310 & 0.265 & 0.207 & 3.775 \\
\hline 1 & 3.413692 & 0.875275 & 0.421168 & 0.000 & 0.000 & 0.000 \\
\hline 1 & 2.220572 & 0.064728 & 1.474688 & 0.000 & 0.000 & 0.000 \\
\hline 1 & 3.346387 & -0.900276 & 0.486349 & 0.000 & 0.000 & 0.000 \\
\hline
\end{tabular}


Tabela A.9: Parâmetros obtidos para o solvente acetonitrila [108].

\begin{tabular}{|c|c|c|c|c|c|c|}
\hline $\mathrm{N}^{\mathrm{o}}$ atômico & \multicolumn{3}{|c|}{ Coordenadas xyz $(\AA)$} & Cargas(e) & $\epsilon(\mathrm{kcal} / \mathrm{mol})$ & $\sigma(\AA)$ \\
\hline 6 & -1.364478 & -1.245037 & -0.813752 & 0.488 & 0.0998 & 3.40 \\
\hline 7 & -0.445246 & -0.926603 & -0.163741 & -0.514 & 0.0998 & 3.30 \\
\hline 6 & -2.511552 & -1.642399 & -1.624877 & -0.577 & 0.0998 & 3.00 \\
\hline 1 & -2.403100 & -1.250547 & -2.632974 & 0.201 & 0.0200 & 2.20 \\
\hline 1 & -2.573568 & -2.726742 & -1.66873 & 0.201 & 0.0200 & 2.20 \\
\hline 1 & -3.425855 & -1.250548 & -1.186617 & 0.201 & 0.0200 & 2.20 \\
\hline
\end{tabular}

